\title{
Catalytic Enantioselective Synthesis of Morpholinones Enabled by Aza-Benzilic Ester Rearrangement
}

$$
\text { Yu-Ping He, }{ }^{1 \dagger} \mathrm{Hua} \mathrm{Wu},{ }^{1,2 \dagger} \text { Qian Wang }{ }^{1} \text { and Jieping } \mathrm{Zhu}^{1 *}
$$

1. Laboratory of Synthesis and Natural Products, Institute of Chemical Sciences and Engineering, Ecole Polytechnique Fédérale de Lausanne, EPFL-SB-ISIC-LSPN, BCH5304, CH-1015 Lausanne, Switzerland.

2. School of Pharmacy, Shanghai Jiao Tong University, 800 Dongchuan Road, Shanghai 200240, P. R. China

\section{Table of Contents}

1. General information $\quad$ S2

2. Synthesis and characterization data of compounds $\mathbf{3}$ and $\mathbf{6} \quad \mathrm{S} 3$

3. Synthesis and characterization data of compounds $\mathbf{1}$ and $\mathbf{4 b}$ S5

$\begin{array}{ll}\text { 4. Synthetic transformations } & \text { S20 }\end{array}$

5. References $\quad$ S23

$\begin{array}{ll}\text { 6. Control experiments } & \text { S23 }\end{array}$

$\begin{array}{ll}\text { 7. Crystallographic data for } \mathbf{1 m} & \text { S24 }\end{array}$

8. Crystallographic data for $\mathbf{1 k} \quad \mathbf{S 3 6}$

9. Crystallographic data for $7 \quad$ S53

10. Copies of NMR spectra and SFC chromatograms $\quad$ S60 


\section{General information}

NMR spectra were recorded on AV2 400 or AV2 $500 \mathrm{MHz}$ Bruker spectrometers. Chemical shifts are given in ppm. The spectra are calibrated to the residual ${ }^{1} \mathrm{H}$ and ${ }^{13} \mathrm{C}$ signals of the solvents. Multiplicities are abbreviated as follows: singlet (s), doublet (d), triplet (t), quartet (q), doubletdoublet (dd), quintet (quint), sextet (sext), septet (sept), multiplet (m), and broad (b). Infrared spectra were recorded on a JASCO FT/IR-4100 spectrometer. Mass spectra were determined with a Waters ACQUITY H-class UPLC/MS ACQ-SQD by electron ionization (EI positive and negative) or a Finnigan TSQ7000 by electrospray ionization (ESI+). The accurate masses were measured by the mass spectrometry service of the EPFL by ESI-TOF using a QTOF Ultima from Waters or APPI-FT-ICR using a linear ion trap Fourier transform ion cyclotron resonance mass spectrometer from Thermo Scientific. Optical rotations $\alpha_{D}$ were obtained with a Jasco P-2000 polarimeter (589 $\mathrm{nm})$. Enantiomeric excesses were determined with a Thar SFC Investigator system using chiral stationary phase columns by comparing the samples with the appropriate racemic samples, column and elution details specified in each entry. Melting points were measured using a Stuart SMP30.

Materials and Methods: Unless otherwise stated, starting materials were purchased from commercial sources (Aldrich, Acros, Merck, Fluka and VWR international). More sensitive compounds were stored in a desiccator or in a glove-box if required. Solvents were purchased in HPLC quality, degassed by purging thoroughly with nitrogen and dried over activated molecular sieves of appropriate size. Alternatively, they were purged with argon and passed through alumina columns in a solvent purification system (Innovative Technology). Reactions were monitored by thin layer chromatography (TLC) using Merck TLC silica gel 60 F254. Compounds were visualized by UV-light at $254 \mathrm{~nm}$ and by dipping the plates in an ethanolic vanillin/sulfuric acid solution or an aqueous potassium permanganate solution followed by heating. Flash column chromatography was performed over silica gel (230-400 mesh). The $\mathrm{CDCl}_{3}$ used in the NMR experiments was stored over anhydrous $\mathrm{K}_{2} \mathrm{CO}_{3}$ before use. 


\section{Synthesis and characterization data of compounds 3 and 6}

All the substrates $\mathbf{3}$ were synthesized according to the reported procedure. ${ }^{[\mathrm{a}]}$

\section{2-(o-tolylamino)ethan-1-ol (3b)}

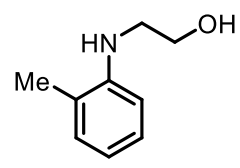

Brown oil, $85 \%$ yield.

${ }^{1}$ H NMR (400 MHz, Chloroform- $d$ ) $\delta 7.15(\mathrm{td}, J=7.7,1.7 \mathrm{~Hz}, 1 \mathrm{H}), 7.09$ (dd, $\left.J=7.4,1.5 \mathrm{~Hz}, 1 \mathrm{H}\right)$, $6.72(\mathrm{td}, J=7.4,1.2 \mathrm{~Hz}, 1 \mathrm{H}), 6.66(\mathrm{dd}, J=8.1,1.2 \mathrm{~Hz}, 1 \mathrm{H}), 3.86(\mathrm{t}, J=5.2 \mathrm{~Hz}, 2 \mathrm{H}), 3.34(\mathrm{t}, J=5.2$ $\mathrm{Hz}, 2 \mathrm{H}), 2.19$ (s, 3H).

${ }^{13}$ C NMR (101 MHz, Chloroform- $d$ ) $\delta$ 146.1, 130.3, 127.2, 122.7, 117.6, 110.2, 61.2, 46.1, 17.6.

IR $\left(v_{\max }, \mathrm{cm}^{-1}\right) 2157,1606,1585,1509,1454,1319,1257,1137,1041,896,750$.

HRMS (ESI/QTOF) m/z: [M + H] $]^{+}$Calcd for $\mathrm{C}_{9} \mathrm{H}_{14} \mathrm{NO}^{+}$152.1070; Found 152.1070.

\section{2-((4-fluorophenyl)amino)ethan-1-ol (3m)}

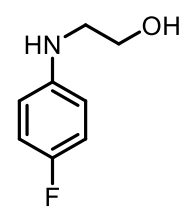

Brown solid, M.p $=55-57{ }^{\circ} \mathrm{C} .88 \%$ yield.

${ }^{1}$ H NMR (400 MHz, Chloroform- $d$ ) $\delta 6.92-6.86(\mathrm{~m}, 2 \mathrm{H}), 6.62-6.56(\mathrm{~m}, 2 \mathrm{H}), 3.81$ (td, $J=5.6,1.6$ $\mathrm{Hz}, 2 \mathrm{H}), 3.24(\mathrm{td}, J=5.2,1.7 \mathrm{~Hz}, 2 \mathrm{H})$.

${ }^{13}$ C NMR (101 MHz, Chloroform- $d$ ) $\delta 156.2(\mathrm{~d}, J=235.5 \mathrm{~Hz}), 144.6(\mathrm{~d}, J=1.7 \mathrm{~Hz}), 115.8(\mathrm{~d}, J=$ $22.3 \mathrm{~Hz}), 114.3(\mathrm{~d}, J=7.2 \mathrm{~Hz}), 61.3,46.9$.

${ }^{19}$ F NMR (377 MHz, Chloroform- $d$ ) $\delta-127.40$.

IR $\left(v_{\max }, \mathrm{cm}^{-1}\right) 3307,2844,1513,1263,1214,1116,1052,908,825,750,715$.

HRMS (ESI/QTOF) m/z: $[\mathrm{M}+\mathrm{H}]^{+}$Calcd for $\mathrm{C}_{8} \mathrm{H}_{11} \mathrm{FNO}^{+}$156.0819; Found 156.0817.

\section{2-((4-chlorophenyl)amino)ethan-1-ol (3p)}

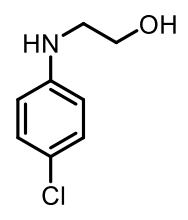

White solid, M.p $=73-75{ }^{\circ} \mathrm{C} .89 \%$ yield. 
${ }^{1}$ H NMR (400 MHz, Chloroform- $d$ ) $\delta 7.13-7.09(\mathrm{~m}, 2 \mathrm{H}), 6.57-6.54(\mathrm{~m}, 2 \mathrm{H}), 3.79(\mathrm{t}, J=4.8 \mathrm{~Hz}$, $2 \mathrm{H}), 3.23(\mathrm{t}, J=5.2 \mathrm{~Hz}, 2 \mathrm{H})$.

${ }^{13}$ C NMR (101 MHz, Chloroform- $d$ ) $\delta$ 146.8, 129.2, 122.5, 114.4, 61.2, 46.2.

IR $\left(v_{\max }, \mathrm{cm}^{-1}\right)$ 3278, 2940, 2161, 1600, 1492, 1309, 1255, 1058, 908, 815, 719.

HRMS (ESI/QTOF) m/z: $[\mathrm{M}+\mathrm{H}]^{+}$Calcd for $\mathrm{C}_{8} \mathrm{H}_{11} \mathrm{CINO}^{+}$172.0524; Found 172.0521.

2-((2-methoxyphenyl)amino)ethan-1-ol (3q)

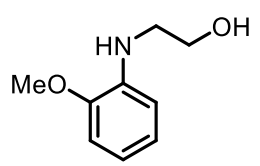

Brown oil, $93 \%$ yield.

${ }^{1}$ H NMR (400 MHz, Chloroform- $d$ ) $\delta 6.88(\mathrm{td}, J=7.6,1.5 \mathrm{~Hz}, 1 \mathrm{H}), 6.79(\mathrm{dd}, J=7.9,1.4 \mathrm{~Hz}, 1 \mathrm{H})$, $6.70(\mathrm{td}, J=7.7,1.6 \mathrm{~Hz}, 1 \mathrm{H}), 6.66(\mathrm{dd}, J=7.8,1.5 \mathrm{~Hz}, 1 \mathrm{H}), 4.49(\mathrm{~s}, 1 \mathrm{H}), 3.85(\mathrm{~s}, 3 \mathrm{H}), 3.85(\mathrm{t}, J=$ $5.2 \mathrm{~Hz}, 2 \mathrm{H}), 3.34(\mathrm{t}, J=5.2 \mathrm{~Hz}, 2 \mathrm{H})$.

${ }^{13}$ C NMR (101 MHz, Chloroform- $d$ ) $\delta$ 147.3, 138.1, 121.4, 117.2, 110.4, 109.7, 61.5, 55.5, 46.1.

IR $\left(v_{\max }, \mathrm{cm}^{-1}\right)$ 2942, 2154, 1594, 1509, 1455, 1346, 1245, 1222, 1132, 1052, 1020, 908.734.

HRMS (ESI/QTOF) m/z: [M + H] $]^{+}$Calcd for $\mathrm{C}_{9} \mathrm{H}_{14} \mathrm{NO}_{2}{ }^{+}$168.1019; Found 168.1017.

\section{2-((2,4-dimethoxyphenyl)amino)ethan-1-ol (3s)}

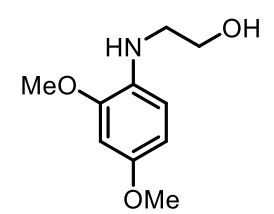

Brown solid, M.p $=61-63{ }^{\circ} \mathrm{C} .87 \%$ yield.

${ }^{1}$ H NMR (400 MHz, Chloroform- $d$ ) $\delta 6.58(\mathrm{~d}, J=8.4 \mathrm{~Hz}, 1 \mathrm{H}), 6.46(\mathrm{~d}, J=2.3 \mathrm{~Hz}, 1 \mathrm{H}), 6.41(\mathrm{dd}, J$ $=8.5,2.2 \mathrm{~Hz}, 1 \mathrm{H}), 3.82(\mathrm{~s}, 3 \mathrm{H}), 3.84-3.79(\mathrm{~m}, 2 \mathrm{H}), 3.76(\mathrm{~s}, 3 \mathrm{H}), 3.28-3.24(\mathrm{~m}, 2 \mathrm{H}), 3.11(\mathrm{br} \mathrm{s}$, $2 \mathrm{H})$.

${ }^{13}$ C NMR (101 MHz, Chloroform- $d$ ) $\delta$ 152.5, 148.5, 132.3, 111.2, 103.9, 99.3, 61.5, 55.9, 55.6, 47.0. IR $\left(v_{\max }, \mathrm{cm}^{-1}\right) 2157,1509,1459,1203,1149,1041,906,836,790,713$.

HRMS (ESI/QTOF) m/z: [M+ H] ${ }^{+}$Calcd for $\mathrm{C}_{10} \mathrm{H}_{16} \mathrm{NO}_{3}{ }^{+}$198.1125; Found 198.1128.

(R)-1-(o-tolylamino)propan-2-ol (6) 


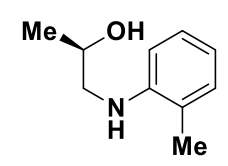

Pale yellow oil, $85 \%$ yield.

${ }^{1} \mathbf{H}$ NMR (400 MHz, Chloroform- $d$ ) $\delta 7.14$ (td, $J=7.8,1.6 \mathrm{~Hz}, 1 \mathrm{H}$ ), 7.09 (br d, $J=7.4,1.5 \mathrm{~Hz}, 1 \mathrm{H}$ ), $6.71(\mathrm{td}, J=7.4,1.1 \mathrm{~Hz}, 1 \mathrm{H}), 6.65$ (br d, $J=8.0,1 \mathrm{H}), 4.11-4.04$ (m, 1H), 3.28 (dd, $J=12.8,3.4 \mathrm{~Hz}$, $1 \mathrm{H}), 3.05(\mathrm{dd}, J=12.8,8.5 \mathrm{~Hz}, 1 \mathrm{H}), 2.19(\mathrm{~s}, 3 \mathrm{H}), 1.30(\mathrm{~d}, J=6.3 \mathrm{~Hz}, 3 \mathrm{H})$.

${ }^{13}$ C NMR (101 MHz, Chloroform- $d$ ) $\delta$ 146.2, 130.3, 127.2, 122.6, 117.6, 110.3, 66.4, 51.7, 21.0, 17.6.

IR $\left(v_{\max }, \mathrm{cm}^{-1}\right) 2973,2161,1600,1585,1508,1444,1375,1319,1255,1143,1126,1051,933,833$, $750,713$.

HRMS (ESI/QTOF) m/z: [M + H] $]^{+}$Calcd for $\mathrm{C}_{10} \mathrm{H}_{16} \mathrm{NO}^{+}$166.1226; Found 166.1226 .

(S)-1-(o-tolylamino)propan-2-ol (6)

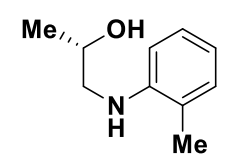

Pale yellow oil, $88 \%$ yield.

${ }^{1}$ H NMR (400 MHz, Chloroform- $d$ ) $\delta 7.14$ (br t, $\left.J=7.7 \mathrm{~Hz}, 1 \mathrm{H}\right), 7.08$ (br d, $J=7.2 \mathrm{~Hz}, 1 \mathrm{H}$ ), 6.70 (br t, $J=7.4 \mathrm{~Hz}, 1 \mathrm{H}), 6.65($ br d, $J=8.0 \mathrm{~Hz}, 1 \mathrm{H}), 4.11-4.04(\mathrm{~m}, 1 \mathrm{H}), 3.28$ (dd, $J=12.8,3.3 \mathrm{~Hz}$, $1 \mathrm{H}), 3.05$ (dd, $J=12.9,8.5 \mathrm{~Hz}, 1 \mathrm{H}), 2.18(\mathrm{~s}, 3 \mathrm{H}), 1.30(\mathrm{~d}, J=6.2 \mathrm{~Hz}, 3 \mathrm{H})$.

${ }^{13}$ C NMR (101 MHz, Chloroform- $d$ ) $\delta$ 146.2, 130.3, 127.2, 122.6, 117.6, 110.3, 66.4, 51.7, 21.0, 17.6.

IR $\left(v_{\max }, \mathrm{cm}^{-1}\right) 2965,2148,1604,1579,1508,1448,1311,1263,1126,1045,923,746$.

HRMS (ESI/QTOF) m/z: [M + H] $]^{+}$Calcd for $\mathrm{C}_{10} \mathrm{H}_{16} \mathrm{NO}^{+}$166.1226; Found 166.1224.

\section{Synthesis and characterization data of compounds 1}

A $10 \mathrm{~mL}$ reaction tube was charged with chiral phosphoric acid (10 mol\%), 2 (1.1 equiv, $0.11 \mathrm{~mol}), 3$ (1.0 equiv, $0.10 \mathrm{~mol})$ and $5 \AA$ molecular sieves $(30 \mathrm{mg})$, TBME $(0.8 \mathrm{~mL})$ were added sequentially. The reaction mixture was then stirred at $80{ }^{\circ} \mathrm{C}$. After completion of the reaction (monitored by TLC), the reaction mixture was concentrated under vacuum and purified by column chromatography on silica gel, eluting with EtOAc/PE to afford the products 1.

(S)-3,4-diphenylmorpholin-2-one (1a) 


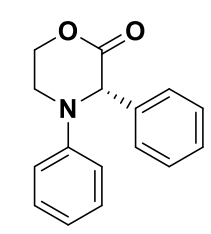

Reaction time: $96 \mathrm{~h}$.

$20.5 \mathrm{mg}$, colorless oil, $80 \%$ yield, $88 \%$ ee.

${ }^{1}$ H NMR (400 MHz, Chloroform- $d$ ) $\delta 7.51$ (d, $\left.J=7.6 \mathrm{~Hz}, 2 \mathrm{H}\right), 7.42-7.34$ (m, 3H), 7.30 - 7.23 (m, 2H), $6.85(\mathrm{t}, J=7.3 \mathrm{~Hz}, 1 \mathrm{H}), 6.66(\mathrm{~d}, J=8.3 \mathrm{~Hz}, 2 \mathrm{H}), 5.57(\mathrm{~s}, 1 \mathrm{H}), 4.50-4.44(\mathrm{~m}, 2 \mathrm{H}), 3.80-3.71$ $(\mathrm{m}, 2 \mathrm{H})$.

${ }^{13}$ C NMR (101 MHz, Chloroform- $d$ ) $\delta 168.5,147.2,136.1,129.7,129.4,128.6,126.2,118.9,112.2$, $64.6,63.7,43.5$.

IR $\left(v_{\max }, \mathrm{cm}^{-1}\right) 1754,1745,1597,1498,1381,1272,1203,1071,996,757$.

HRMS (ESI/QTOF) m/z: [M + H] $]^{+}$Calcd for $\mathrm{C}_{16} \mathrm{H}_{16} \mathrm{NO}_{2}{ }^{+}$254.1176; Found 254.1181. $[\alpha]^{26} \mathrm{D}+43.4($ c 1.0, DCM).

SFC: IA column, $5 \% \mathrm{MeOH}$ in supercritical $\mathrm{CO}_{2}$ as eluent, $4 \mathrm{~mL} / \mathrm{min}$. $\mathrm{tR}=6.32 \mathrm{~min}$ (minor), 8.22 $\min$ (major).

\section{(S) -3-phenyl-4-(o-tolyl)morpholin-2-one (1b)}

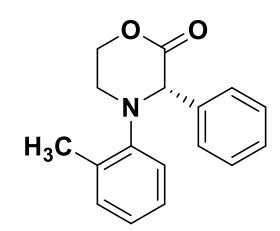

Reaction time: $16 \mathrm{~h}$.

$22.3 \mathrm{mg}$, colorless oil, $85 \%$ yield, $92 \%$ ee.

${ }^{1}$ H NMR (400 MHz, Chloroform- $d$ ) $\delta 7.37$ - $7.31(\mathrm{~m}, 2 \mathrm{H}), 7.30$ - $7.21(\mathrm{~m}, 3 \mathrm{H}), 7.20$ - $7.14(\mathrm{~m}, 1 \mathrm{H})$, $7.11(\mathrm{td}, J=7.6,1.7 \mathrm{~Hz}, 1 \mathrm{H}), 7.05-6.99(\mathrm{~m}, 2 \mathrm{H}), 5.19(\mathrm{~s}, 1 \mathrm{H}), 4.66(\mathrm{ddd}, J=10.9,8.5,3.5 \mathrm{~Hz}, 1 \mathrm{H})$, 4.51 (ddd, $J=10.9,4.8,3.6 \mathrm{~Hz}, 1 \mathrm{H}), 3.40$ (ddd, $J=13.4,4.8,3.5 \mathrm{~Hz}, 1 \mathrm{H}), 3.20$ (ddd, $J=13.3,8.5$, $3.7 \mathrm{~Hz}, 1 \mathrm{H}), 2.35$ (s, 3H).

${ }^{13}$ C NMR (101 MHz, Chloroform- $d$ ) $\delta$ 169.5, 147.6, 136.6, 134.1, 131.5, 128.5, 128.34, 128.2, 126.8, 125.2, 122.0, 68.4, 67.5, 48.1, 18.2.

HRMS (ESI/QTOF) m/z: [M + H] $]^{+}$Calcd for $\mathrm{C}_{17} \mathrm{H}_{18} \mathrm{NO}_{2}{ }^{+}$268.1332; Found 268.1333.

IR $\left(v_{\max }, \mathrm{cm}^{-1}\right) 1754,1745,1597,1498,1381,1272,1203,1071,996,757$. $[\alpha]^{26}{ }_{\mathrm{D}}+52.8(c 1.0, \mathrm{DCM})$.

SFC: AD-H column, $5 \% \mathrm{MeOH}$ in supercritical $\mathrm{CO}_{2}$ as eluent, $4 \mathrm{~mL} / \mathrm{min}$. $\mathrm{tR}=5.63 \mathrm{~min}$ (minor), 6.67 min (major). 
(S)-3-([1,1'-biphenyl]-4-yl)-4-(o-tolyl)morpholin-2-one (1c)

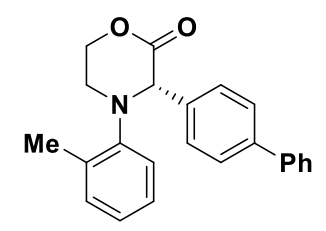

Reaction time: $16 \mathrm{~h}$.

$31.9 \mathrm{mg}$, pale yellow foam, $93 \%$ yield, $90 \%$ ee.

${ }^{1}$ H NMR (400 MHz, Chloroform- $\left.d\right) \delta 7.56-7.51(\mathrm{~m}, 2 \mathrm{H}), 7.51-7.46(\mathrm{~m}, 2 \mathrm{H}), 7.44-7.38(\mathrm{~m}, 4 \mathrm{H})$, $7.36-7.29(\mathrm{~m}, 1 \mathrm{H}), 7.18(\mathrm{~d}, J=7.5 \mathrm{~Hz}, 1 \mathrm{H}), 7.15-7.10(\mathrm{~m}, 1 \mathrm{H}), 7.07-7.00(\mathrm{~m}, 2 \mathrm{H}), 5.23(\mathrm{~s}, 1 \mathrm{H})$, 4.69 (ddd, $J=11.0,8.5,3.5 \mathrm{~Hz}, 1 \mathrm{H}), 4.53(\mathrm{ddd}, J=11.0,4.8,3.6 \mathrm{~Hz}, 1 \mathrm{H}), 3.42$ (ddd, $J=13.4,4.8$, $3.5 \mathrm{~Hz}, 1 \mathrm{H}), 3.21$ (ddd, $J=13.4,8.6,3.6 \mathrm{~Hz}, 1 \mathrm{H}), 2.37$ (s, 3H).

${ }^{13}$ C NMR (101 MHz, Chloroform- $d$ ) $\delta$ 169.5, 147.6, 141.0, 140.6, 135.7, 134.1, 131.5, 128.9, 128.8, $127.5,127.2,127.2,126.9,125.2,121.9,68.4,67.2,48.2,18.3$.

IR $\left(v_{\max }, \mathrm{cm}^{-1}\right) 2910,2352,2011,1727,1486,1369,1270,1203,1078,1002,734,701$.

HRMS (ESI/QTOF) m/z: [M + H] ${ }^{+}$Calcd for $\mathrm{C}_{23} \mathrm{H}_{22} \mathrm{NO}_{2}{ }^{+} 344.1645$; Found 344.1650. $[\alpha]^{26} \mathrm{D}+86.2(c 1.0, \mathrm{DCM})$.

SFC: IA column, $30 \% \mathrm{MeOH}$ in supercritical $\mathrm{CO}_{2}$ as eluent, $4 \mathrm{~mL} / \mathrm{min}$. $\mathrm{tR}=3.71 \mathrm{~min}$ (major), 4.89 $\min (\operatorname{minor})$.

(S)-3-(2-fluorophenyl)-4-(o-tolyl)morpholin-2-one (1d)

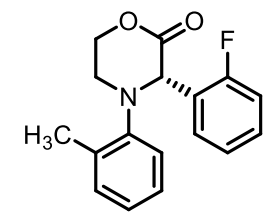

Reaction time: $36 \mathrm{~h}$.

$21.1 \mathrm{mg}$, brown foam, $74 \%$ yield, $85 \%$ ee.

${ }^{1}$ H NMR (400 MHz, Chloroform- $d$ ) $\delta 7.21-7.14(\mathrm{~m}, 1 \mathrm{H}), 7.13-7.07$ (m, 4H), $7.05-6.89$ (m, 3H), $5.31(\mathrm{~s}, 1 \mathrm{H}), 4.84-4.77(\mathrm{~m}, 1 \mathrm{H}), 4.54(\mathrm{dt}, J=10.8,3.3 \mathrm{~Hz}, 1 \mathrm{H}), 3.29-3.25(\mathrm{~m}, 2 \mathrm{H}), 2.19(\mathrm{~s}, 3 \mathrm{H})$. ${ }^{13}$ C NMR (101 MHz, Chloroform- $\left.d\right) \delta 168.6,161.0(\mathrm{~d}, J=247.9 \mathrm{~Hz}), 147.0,135.2,131.2,130.7$ (d, $J=3.7 \mathrm{~Hz}), 130.1(\mathrm{~d}, J=8.4 \mathrm{~Hz}), 126.8,125.7,124.8(\mathrm{~d}, J=13.4 \mathrm{~Hz}), 124.0(\mathrm{~d}, J=3.6 \mathrm{~Hz}), 121.8$, $115.7(\mathrm{~d}, J=21.8 \mathrm{~Hz}), 69.1,62.5,49.5,17.6$.

${ }^{19}$ F NMR (377 MHz, Chloroform-d) $\delta-116.43$.

IR $\left(v_{\max }, \mathrm{cm}^{-1}\right) 2366,2269,2163,2121,2019,1986,1739,1486,1267,1201,1014,863,763,742$.

HRMS (ESI/QTOF) m/z: [M + H] $]^{+}$Calcd for $\mathrm{C}_{17} \mathrm{H}_{17} \mathrm{FNO}_{2}{ }^{+}$286.1238; Found 286.1235.

$[\alpha]^{25} \mathrm{D}+53.3$ ( c 1.0, DCM). 
HPLC: IA, hexane/i-PrOH $=90 / 10$, flow rate: $1.0 \mathrm{~mL} / \mathrm{min}, \mathrm{T}=30{ }^{\circ} \mathrm{C}, 254 \mathrm{~nm}, 9.61 \mathrm{~min}$ (minor), $11.73 \mathrm{~min}$ (major).

(S)-3-(3-bromophenyl)-4-(o-tolyl)morpholin-2-one (1e)

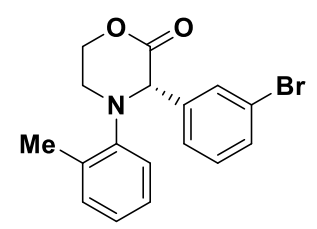

Reaction time: $16 \mathrm{~h}$.

$31.4 \mathrm{mg}$, brown foam, $91 \%$ yield, $90 \%$ ee.

${ }^{1}$ H NMR (400 MHz, Chloroform- $d$ ) $\delta 7.52(\mathrm{t}, J=1.9 \mathrm{~Hz}, 1 \mathrm{H}), 7.35$ (ddd, $\left.J=8.0,2.0,1.1 \mathrm{~Hz}, 1 \mathrm{H}\right)$, $7.28-7.25(\mathrm{~m}, 1 \mathrm{H}), 7.19-7.15(\mathrm{~m}, 1 \mathrm{H}),, 7.14-7.09(\mathrm{~m}, 2 \mathrm{H}),, 7.02(\mathrm{t}, J=7.3 \mathrm{~Hz}, 2 \mathrm{H}), 5.14(\mathrm{~s}$, 1H), 4.67 (ddd, $J=11.0,8.9,3.4 \mathrm{~Hz}, 1 \mathrm{H}), 4.51(\mathrm{ddd}, J=11.0,4.4,3.6 \mathrm{~Hz}, 1 \mathrm{H}), 3.36(\mathrm{ddd}, J=13.4$, 4.4, 3.4 Hz, 1H), 3.20 (ddd, $J=13.1,8.9,3.5 \mathrm{~Hz}, 1 \mathrm{H}), 2.34$ (s, 3H).

${ }^{13}$ C NMR (101 MHz, Chloroform- $d$ ) $\delta$ 168.8, 147.2, 139.0, 134.2, 131.7, 131.43, 131.42, 130.0, $127.2,127.0,125.5,122.6,121.8,68.4,67.0,48.5,18.2$.

IR $\left(v_{\max }, \mathrm{cm}^{-1}\right)$ 2019, 1739, 1604, 1498, 1209, 1133, 1079, 987, 960, 852, 804, 769.

HRMS (ESI/QTOF) m/z: [M + H] ${ }^{+}$Calcd for $\mathrm{C}_{17} \mathrm{H}_{17} \mathrm{BrNO}_{2}{ }^{+} 346.0437$; Found 346.0437. $[\alpha]^{27} \mathrm{D}+65.1(c$ 1.0, DCM).

SFC: IA column, $5 \% \mathrm{MeOH}$ in supercritical $\mathrm{CO}_{2}$ as eluent, $4 \mathrm{~mL} / \mathrm{min}$. $\mathrm{tR}=7.52 \mathrm{~min}$ (minor), 8.42 $\min$ (major).

(S)-4-(o-tolyl)-3-(4-(trifluoromethyl)phenyl)morpholin-2-one (1f)

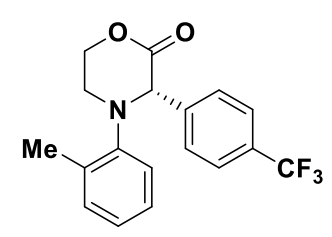

Reaction time: $24 \mathrm{~h}$.

$28.7 \mathrm{mg}$, brown foam, $85 \%$ yield, $86 \%$ ee.

${ }^{1}$ H NMR (400 MHz, Chloroform-d) $\delta 7.55$ - 7.46 (m, 4H), 7.20 - 7.15 (m, 1H), 7.14 - 7.08 (m, $1 \mathrm{H}), 7.06-6.99(\mathrm{~m}, 2 \mathrm{H}), 5.25(\mathrm{~s}, 1 \mathrm{H}), 4.70(\mathrm{ddd}, J=10.9,9.0,3.4 \mathrm{~Hz}, 1 \mathrm{H}), 4.53(\mathrm{dt}, J=11.0,3.8$ $\mathrm{Hz}, 1 \mathrm{H}), 3.36(\mathrm{dt}, J=13.5,3.8 \mathrm{~Hz}, 1 \mathrm{H}), 3.23(\mathrm{ddd}, J=13.2,9.1,3.5 \mathrm{~Hz}, 1 \mathrm{H}), 2.34(\mathrm{~s}, 3 \mathrm{H})$.

${ }^{13}$ C NMR (101 MHz, Chloroform- $d$ ) $\delta$ 168.6, 147.0, 140.8 (q, $\left.J=1.2 \mathrm{~Hz}\right), 134.1,131.7,130.4$ (q, $J$ $=32.4 \mathrm{~Hz}), 128.9,127.0,125.54,125.48(\mathrm{q}, J=3.8 \mathrm{~Hz}), 124.0(\mathrm{q}, J=273.4 \mathrm{~Hz}), 121.6,68.5,67.2$, $48.8,18.1$. 
IR $\left(v_{\max }, \mathrm{cm}^{-1}\right) 1737,1486,1324,1213,1164,1110,1062,998,821,757,723,680$.

HRMS (ESI/QTOF) m/z: [M + H] ${ }^{+}$Calcd for $\mathrm{C}_{18} \mathrm{H}_{17} \mathrm{~F}_{3} \mathrm{NO}_{2}{ }^{+}$336.1206; Found 336.1208.

$[\alpha]^{21} \mathrm{D}+32.8(c$ 1.0, DCM).

SFC: IA column, $5 \% \mathrm{MeOH}$ in supercritical $\mathrm{CO}_{2}$ as eluent, $4 \mathrm{~mL} / \mathrm{min}$. $\mathrm{tR}=3.41 \mathrm{~min}$ (minor), 4.04 $\min$ (major).

(S)-3-(2,6-difluorophenyl)-4-(o-tolyl)morpholin-2-one (1g)

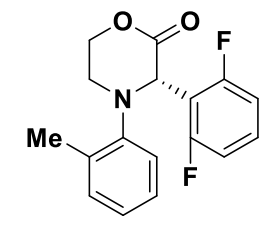

Reaction time: $20 \mathrm{~h}$.

$28.8 \mathrm{mg}$, brown foam, $95 \%$ yield, $87 \%$ ee.

${ }^{1}$ H NMR (400 MHz, Chloroform- $d$ ) $\delta 7.15-6.98(\mathrm{~m}, 5 \mathrm{H}), 6.76-6.63(\mathrm{~m}, 2 \mathrm{H}), 5.27(\mathrm{~s}, 1 \mathrm{H}), 4.80$ (ddd, $J=10.7,8.3,4.9 \mathrm{~Hz}, 1 \mathrm{H}), 4.54(\mathrm{dt}, J=10.8,3.2 \mathrm{~Hz}, 1 \mathrm{H}), 3.31-3.22(\mathrm{~m}, 2 \mathrm{H}), 2.20(\mathrm{~s}, 3 \mathrm{H})$.

${ }^{13}$ C NMR (101 MHz, Chloroform- $d$ ) $\delta 168.4,162.8(\mathrm{dd}, J=249.7,12.2 \mathrm{~Hz}), 161.2(\mathrm{dd}, J=250.6$, $12.2 \mathrm{~Hz}), 146.8,135.2,131.41(\mathrm{dd}, J=9.8 \mathrm{~Hz}, 5.2 \mathrm{~Hz}), 131.4,126.9,125.9,121.7,120.9(\mathrm{dd}, J=$ 13.5, 4.0 Hz), $111.3(\mathrm{dd}, J=21.4,3.7 \mathrm{~Hz}), 104.2(\mathrm{t}, J=25.7 \mathrm{~Hz}), 69.1$, 62.1, 49.8, 17.6.

${ }^{19}$ F NMR (377 MHz, Chloroform- $d$ ) $\delta$-109.57, -112.19.

IR $\left(v_{\max }, \mathrm{cm}^{-1}\right)$ 2019, 1739, 1604, 1498, 1209, 1133, 1079, 987, 852, 804, 709.

HRMS (ESI/QTOF) m/z: [M + H] ${ }^{+}$Calcd for $\mathrm{C}_{17} \mathrm{H}_{16} \mathrm{~F}_{2} \mathrm{NO}_{2}{ }^{+}$304.1144; Found 304.1143.

$[\alpha]^{27} \mathrm{D}+52.3(c 1.0, \mathrm{DCM})$.

SFC: IC column, $1.5 \% \mathrm{MeOH}$ in supercritical $\mathrm{CO}_{2}$ as eluent, $4 \mathrm{~mL} / \mathrm{min}$. $\mathrm{tR}=15.01 \mathrm{~min}$ (minor), $16.92 \min$ (major).

(S)-3-(naphthalen-2-yl)-4-(o-tolyl)morpholin-2-one (1h)

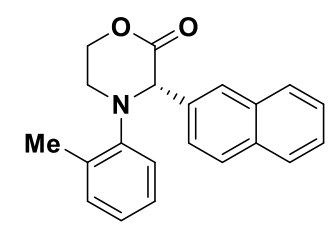

Reaction time: $20 \mathrm{~h}$.

$26.1 \mathrm{mg}$, colorless foam, $84 \%$ yield, $92 \%$ ee.

${ }^{1}$ H NMR (400 MHz, Chloroform- $d$ ) $\delta 7.79-7.73(\mathrm{~m}, 4 \mathrm{H}), 7.51-7.41(\mathrm{~m}, 3 \mathrm{H}), 7.14(\mathrm{~d}, J=7.4 \mathrm{~Hz}$, 1H), $7.12-7.07(\mathrm{~m}, 2 \mathrm{H}), 7.01-6.96(\mathrm{~m}, 1 \mathrm{H}), 5.34(\mathrm{~s}, 1 \mathrm{H}), 4.71(\mathrm{ddd}, J=10.9,8.6,3.5 \mathrm{~Hz}, 1 \mathrm{H})$, 
$4.54(\mathrm{ddd}, J=11.0,4.8,3.6 \mathrm{~Hz}, 1 \mathrm{H}), 3.43$ (ddd, $J=13.4,4.8,3.5 \mathrm{~Hz}, 1 \mathrm{H}), 3.23$ (ddd, $J=13.4,8.6$, $3.6 \mathrm{~Hz}, 1 \mathrm{H}), 2.37$ (s, 3H).

${ }^{13}$ C NMR (101 MHz, Chloroform-d) $\delta$ 169.5, 147.6, 134.21, 134.15, 133.2, 133.1, 131.6, 128.4, $128.2,127.9,127.7,126.9,126.33,126.29,125.9,125.3,122.0,68.4,67.6,48.3,18.3$.

IR $\left(v_{\max }, \mathrm{cm}^{-1}\right) 2308,2161,2017,1737,1490,1203,1083,815,761,723$.

HRMS (ESI/QTOF) m/z: [M + H] $]^{+}$Calcd for $\mathrm{C}_{21} \mathrm{H}_{20} \mathrm{NO}_{2}{ }^{+} 318.1489$; Found 318.1489. $[\alpha]^{29} \mathrm{D}+80.1$ ( $c$ 1.0, DCM).

SFC: OD-H column, $15 \% \mathrm{MeOH}$ in supercritical $\mathrm{CO}_{2}$ as eluent, $4 \mathrm{~mL} / \mathrm{min}$. $\mathrm{tR}=6.26 \mathrm{~min}$ (minor), 7.94 min (major).

(S)-3-(benzo[d][1,3]dioxol-5-yl)-4-(o-tolyl)morpholin-2-one (1i)

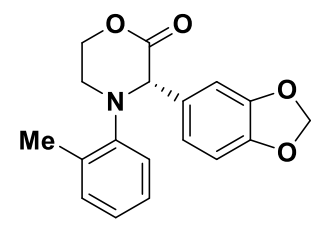

Reaction time: $24 \mathrm{~h}$.

$27.4 \mathrm{mg}$, colorless foam, $88 \%$ yield, $86 \%$ ee.

${ }^{1}$ H NMR (400 MHz, Chloroform- $d$ ) $\delta 7.18-7.08(\mathrm{~m}, 2 \mathrm{H}), 7.04-6.98$ (m, 2H), 6.83 (d, J = 1.8 Hz, 1H), 6.79 (dd, $J=8.2,1.8 \mathrm{~Hz}, 1 \mathrm{H}), 6.67$ (d, $J=8.0 \mathrm{~Hz}, 1 \mathrm{H}), 5.89$ (s, 2H), 5.08 (s, 1H), 4.67 (ddd, $J=$ $10.9,9.0,3.5 \mathrm{~Hz}, 1 \mathrm{H}), 4.49$ (ddd, $J=10.9,4.3,3.5 \mathrm{~Hz}, 1 \mathrm{H}), 3.36$ (ddd, $J=13.4,4.3,3.4 \mathrm{~Hz}, 1 \mathrm{H}$ ), 3.17 (ddd, $J=13.2,9.0,3.6 \mathrm{~Hz}, 1 \mathrm{H}), 2.34$ (s, 3H).

${ }^{13}$ C NMR (101 MHz, Chloroform- $d$ ) $\delta$ 169.5, 147.8, 147.54, 147.48, 134.2, 131.5, 130.5, 126.8, $125.2,122.2,121.8,108.6,108.2,101.3,68.5,67.3,48.6,18.3$.

HRMS (ESI/QTOF) m/z: [M + H] ${ }^{+}$Calcd for $\mathrm{C}_{18} \mathrm{H}_{18} \mathrm{NO}_{4}{ }^{+}$312.1230; Found 312.1228.

IR $\left(v_{\max }, \mathrm{cm}^{-1}\right)$ 2902, 2356, 2022, 1743, 1486, 1434, 1245, 1207, 1091, 1031, 921, 804, 767. $[\alpha]^{25} \mathrm{D}+88.3(c 1.0, \mathrm{DCM})$.

SFC: IC column, $10 \% \mathrm{MeOH}$ in supercritical $\mathrm{CO}_{2}$ as eluent, $4 \mathrm{~mL} / \mathrm{min}$. $\mathrm{tR}=6.34 \mathrm{~min}$ (minor), 7.27 $\min$ (major).

(S)-3-(thiophen-3-yl)-4-(o-tolyl)morpholin-2-one (1j)<smiles>Cc1ccccc1N1CCOC(=O)[C@H]1c1ccsc1</smiles>

Reaction time: $16 \mathrm{~h}$. 
$22.0 \mathrm{mg}$, brown oil, $81 \%$ yield, $83 \%$ ee.

${ }^{1}$ H NMR (400 MHz, Chloroform- $d$ ) $\delta 7.23-7.17(\mathrm{~m}, 2 \mathrm{H}), 7.15-7.10(\mathrm{~m}, 2 \mathrm{H}), 7.04(\mathrm{td}, J=7.4,1.3$ $\mathrm{Hz}, 1 \mathrm{H}), 6.98(\mathrm{dd}, J=5.0,1.3 \mathrm{~Hz}, 1 \mathrm{H}), 6.92(\mathrm{dd}, J=7.9,1.3 \mathrm{~Hz}, 1 \mathrm{H}), 5.22(\mathrm{~s}, 1 \mathrm{H}), 4.59-4.47(\mathrm{~m}$, 2H), 3.46 (ddd, $J=13.5,6.6,3.9 \mathrm{~Hz}, 1 \mathrm{H}), 3.15-3.08(\mathrm{~m}, 1 \mathrm{H}), 2.36(\mathrm{~s}, 3 \mathrm{H})$.

${ }^{13}$ C NMR (101 MHz, Chloroform- $d$ ) $\delta$ 170.0, 147.5, 136.8, 133.7, 131.6, 127.4, 126.9, 125.9, 125.1, $123.9,121.9,68.2,63.0,46.5,18.2$.

IR $\left(v_{\max }, \mathrm{cm}^{-1}\right) 2148,2024,1739,1492,1203,1002,838,752$.

HRMS (ESI/QTOF) m/z: [M + H] ${ }^{+}$Calcd for $\mathrm{C}_{15} \mathrm{H}_{16} \mathrm{NO}_{2} \mathrm{~S}^{+}$274.0896; Found 274.0900. $[\alpha]^{23} \mathrm{D}+38.1$ ( c 1.0, DCM).

SFC: AD-H column, $5 \% \mathrm{MeOH}$ in supercritical $\mathrm{CO}_{2}$ as eluent, $4 \mathrm{~mL} / \mathrm{min}$. $\mathrm{tR}=7.24 \mathrm{~min}$ (minor), $8.12 \mathrm{~min}$ (major).

(S)-4-(o-tolyl)-3-(1-tosyl-1H-indol-3-yl)morpholin-2-one (1k)

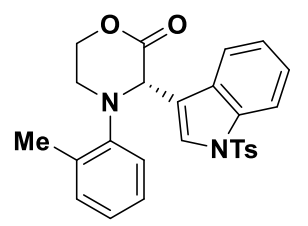

Reaction time: $4 \mathrm{~h}$.

$23.6 \mathrm{mg}$, white solid, $51 \%$ yield, $81 \%$ ee. M.p $=146-148^{\circ} \mathrm{C}$.

${ }^{1}$ H NMR (400 MHz, Chloroform- $\left.d\right) \delta 7.84(\mathrm{~d}, J=8.2 \mathrm{~Hz}, 1 \mathrm{H}), 7.50-7.42(\mathrm{~m}, 4 \mathrm{H}), 7.23(\mathrm{~d}, J=7.7$ $\mathrm{Hz}, 1 \mathrm{H}), 7.19-7.09(\mathrm{~m}, 4 \mathrm{H}), 7.09-7.00(\mathrm{~m}, 2 \mathrm{H}), 6.98-6.94(\mathrm{~m}, 1 \mathrm{H}), 5.35(\mathrm{~s}, 1 \mathrm{H}), 4.75(\mathrm{ddd}, J=$ $11.2,7.9,3.4 \mathrm{~Hz}, 1 \mathrm{H}), 4.57$ (ddd, $J=11.0,5.3,3.5 \mathrm{~Hz}, 1 \mathrm{H}), 3.41$ (ddd, $J=13.8,5.4,3.5 \mathrm{~Hz}, 1 \mathrm{H}$ ), 3.19 (ddd, $J=13.6,8.0,3.5 \mathrm{~Hz}, 1 \mathrm{H}), 2.32$ (s, 3H), 2.24 (s, 3H).

${ }^{13}$ C NMR (101 MHz, Chloroform- $d$ ) $\delta$ 168.1, 147.2, 145.0, 135.2, 135.1, 134.3, 131.6, 130.0, 129.4, $126.9,126.8,126.3,125.4,125.0,123.4,121.5,120.5,118.2,113.6,68.7,60.2$, 48.1, 21.7, 18.0 .

IR $\left(v_{\max }, \mathrm{cm}^{-1}\right) 1739,1591,1489,1450,1366,1251,1215,1167,1119,1086,987,740$.

HRMS (ESI/QTOF) m/z: [M + H] ${ }^{+}$Calcd for $\mathrm{C}_{26} \mathrm{H}_{25} \mathrm{~N}_{2} \mathrm{O}_{4} \mathrm{~S}^{+} 461.1530$; Found 461.1535. $[\alpha]^{26}+29.2(c 1.0, \mathrm{DCM})$.

SFC: IA column, $10 \% \mathrm{MeOH}$ in supercritical $\mathrm{CO}_{2}$ as eluent, $4 \mathrm{~mL} / \mathrm{min}$. $\mathrm{tR}=13.35 \mathrm{~min}$ (minor), $15.00 \mathrm{~min}$ (major).

(S)-3-cyclohexyl-4-(o-tolyl)morpholin-2-one (11) 


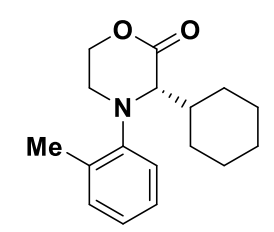

Reaction time: $18 \mathrm{~h}$.

$14.6 \mathrm{mg}$, colorless oil, $53 \%$ yield, $76 \%$ ee.

${ }^{1}$ H NMR (400 MHz, Chloroform- $d$ ) $\delta 7.25-7.18(\mathrm{~m}, 2 \mathrm{H}), 7.14(\mathrm{dd}, J=8.0,1.4 \mathrm{~Hz}, 1 \mathrm{H}), 7.08(\mathrm{td}, J$ $=7.3,1.4 \mathrm{~Hz}, 1 \mathrm{H}), 4.49(\mathrm{ddd}, J=11.3,8.3,3.0 \mathrm{~Hz}, 1 \mathrm{H}), 4.37(\mathrm{ddd}, J=10.9,4.9,3.2 \mathrm{~Hz}, 1 \mathrm{H}), 4.03$ $(\mathrm{d}, J=4.2 \mathrm{~Hz}, 1 \mathrm{H}), 3.21(\mathrm{ddd}, J=13.4,4.9,3.0 \mathrm{~Hz}, 1 \mathrm{H}), 3.03(\mathrm{ddd}, J=13.5,8.3,3.2 \mathrm{~Hz}, 1 \mathrm{H}), 2.34$ (s, 3H), $1.84-1.41(\mathrm{~m}, 7 \mathrm{H}), 1.20-1.01(\mathrm{~m}, 4 \mathrm{H})$.

${ }^{13}$ C NMR (101 MHz, Chloroform- $d$ ) $\delta$ 170.9, 149.4, 135.0, 131.6, 127.2, 125.3, 122.6, 68.4, 67.8, $48.9,42.5,30.1,29.5,26.6,26.4,26.3,18.7$.

IR $\left(v_{\max }, \mathrm{cm}^{-1}\right)$ 2931, 2846, 2362, 2281, 2125, 1743, 1490, 1450, 1201, 1008.

HRMS (ESI/QTOF) m/z: [M + H] ${ }^{+}$Calcd for $\mathrm{C}_{17} \mathrm{H}_{24} \mathrm{NO}_{2}{ }^{+} 274.1802$; Found 274.1802. $[\alpha]^{26} \mathrm{D}-11.7(c 0.5, \mathrm{DCM})$.

HPLC: IB, hexane/i-PrOH = 90/10, flow rate: $1.0 \mathrm{~mL} / \mathrm{min}, \mathrm{T}=30{ }^{\circ} \mathrm{C}, 254 \mathrm{~nm}, 7.03 \mathrm{~min}$ (minor), $7.60 \mathrm{~min}$ (major).

(S)-4-(4-fluorophenyl)-3-phenylmorpholin-2-one (1m)

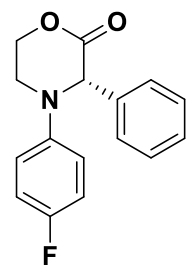

Reaction time: $48 \mathrm{~h}$.

$24.3 \mathrm{mg}$, white solid, $89 \%$ yield, $90 \%$ ee. M.p $=139-141{ }^{\circ} \mathrm{C}$.

${ }^{1}$ H NMR (400 MHz, Chloroform- $d$ ) $\delta 7.52$ - 7.48 (m, 2H), $7.4-7.32$ (m, 3H), 6.99 - 6.91 (m, 2H), $6.60-6.55(\mathrm{~m}, 2 \mathrm{H}), 5.46(\mathrm{~s}, 1 \mathrm{H}), 4.50-4.45(\mathrm{~m}, 2 \mathrm{H}), 3.76-3.68(\mathrm{~m}, 2 \mathrm{H})$.

${ }^{13}$ C NMR (101 MHz, Chloroform- $d$ ) $\delta 168.4,156.7(\mathrm{~d}, J=237.8 \mathrm{~Hz}), 143.8(\mathrm{~d}, J=2.0 \mathrm{~Hz}), 136.1$, 129.4, 128.7, 126.3, 116.2 (d, $J=22.5 \mathrm{~Hz}), 113.4(\mathrm{~d}, J=7.5 \mathrm{~Hz}), 64.7,64.4,43.9$.

${ }^{19}$ F NMR (377 MHz, Chloroform- $d$ ) $\delta-126.24$.

IR $\left(v_{\max }, \mathrm{cm}^{-1}\right) 1742,1508,1269,1224,1203,1071,984,809,754,737$.

HRMS (ESI/QTOF) m/z: [M + H] $]^{+}$Calcd for $\mathrm{C}_{16} \mathrm{H}_{15} \mathrm{FNO}_{2}{ }^{+}$272.1081; Found 272.1080. $[\alpha]^{20} \mathrm{D}+26.9(c$ 1.0, DCM).

SFC: OJ-H column, $12 \% \mathrm{MeOH}$ in supercritical $\mathrm{CO}_{2}$ as eluent, $4 \mathrm{~mL} / \mathrm{min}$. $\mathrm{tR}=3.67 \mathrm{~min}$ (minor), 
$5.38 \mathrm{~min}$ (major).

(S)-4-(4-fluorophenyl)-3-(4-methoxyphenyl)morpholin-2-one (1n)

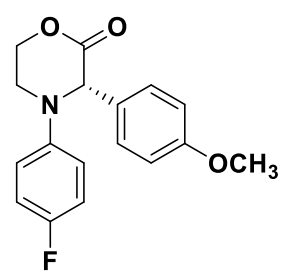

Reaction time: $96 \mathrm{~h}$.

$22.9 \mathrm{mg}$, white foam, $76 \%$ yield, $85 \%$ ee.

${ }^{1}$ H NMR (400 MHz, Chloroform- $d$ ) $\delta 7.42$ - 7.37 (m, 2H), 6.97 - 6.89 (m, 4H), 6.60 - 6.55 (m, 2H), $5.38(\mathrm{~s}, 1 \mathrm{H}), 4.50-4.46(\mathrm{~m}, 2 \mathrm{H}), 3.81(\mathrm{~s}, 3 \mathrm{H}), 3.72-3.62(\mathrm{~m}, 2 \mathrm{H})$.

${ }^{13}$ C NMR (101 MHz, Chloroform- $d$ ) $\delta$ 168.6, 156.0, $156.6(\mathrm{~d}, J=237.7 \mathrm{~Hz}), 143.8(\mathrm{~d}, J=1.9 \mathrm{~Hz})$, 127.8, 127.5, $116.1(\mathrm{~d}, J=22.3 \mathrm{~Hz}), 114.7,113.5(\mathrm{~d}, J=7.5 \mathrm{~Hz}), 64.8,63.9,55.5,43.9$.

${ }^{19}$ F NMR (377 MHz, Chloroform- $d$ ) $\delta-126.30$.

IR $\left(v_{\max }, \mathrm{cm}^{-1}\right) 2160,1796,1752,1607,1513,1462,1272,1234,1215,1077,824$.

HRMS (ESI/QTOF) m/z: [M + H] ${ }^{+}$Calcd for $\mathrm{C}_{17} \mathrm{H}_{17} \mathrm{FNO}_{3}{ }^{+}$302.1187; Found 302.1187. $[\alpha]^{28} \mathrm{D}+39.3(c 1.0, \mathrm{DCM})$.

SFC: IA column, $10 \% \mathrm{MeOH}$ in supercritical $\mathrm{CO}_{2}$ as eluent, $4 \mathrm{~mL} / \mathrm{min}$. $\mathrm{tR}=5.09 \mathrm{~min}$ (minor), 6.18 min (major).

(S)-4-(4-fluorophenyl)-3-(p-tolyl)morpholin-2-one (10)

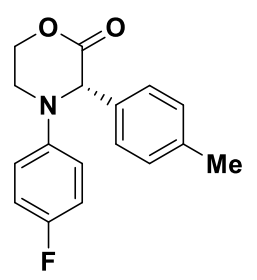

Reaction time: $48 \mathrm{~h}$.

$21.1 \mathrm{mg}$, white foam, $74 \%$ yield, $90 \%$ ee.

${ }^{1} \mathbf{H}$ NMR (400 MHz, Chloroform- $d$ ) $\delta 7.37(\mathrm{~d}, J=8.0 \mathrm{~Hz}, 2 \mathrm{H}), 7.19(\mathrm{~d}, J=8.0 \mathrm{~Hz}, 2 \mathrm{H}), 6.98-6.91$ $(\mathrm{m}, 2 \mathrm{H}), 6.61-6.54(\mathrm{~m}, 2 \mathrm{H}), 5.42(\mathrm{~s}, 1 \mathrm{H}), 4.52-4.42(\mathrm{~m}, 2 \mathrm{H}), 3.74-3.64(\mathrm{~m}, 2 \mathrm{H}), 2.35(\mathrm{~s}, 3 \mathrm{H})$.

${ }^{13}$ C NMR (101 MHz, Chloroform- $\left.d\right) \delta 168.6,156.6(\mathrm{~d}, J=237.6 \mathrm{~Hz}), 143.8(\mathrm{~d}, J=1.9 \mathrm{~Hz}), 138.6$, 133.1, 130.1, 126.2, 116.1 (d, $J=22.5 \mathrm{~Hz}), 113.4$ (d, $J=7.5 \mathrm{~Hz}), 64.7,64.1,43.9,21.2$.

${ }^{19}$ F NMR (377 MHz, Chloroform- $d$ ) $\delta-126.40$. 
IR $\left(v_{\max }, \mathrm{cm}^{-1}\right) 2154,2024,1739,1508,1465,1375,1276,1207,1074,983,817,757$.

HRMS (ESI/QTOF) m/z: [M + H] ${ }^{+}$Calcd for $\mathrm{C}_{17} \mathrm{H}_{17} \mathrm{FNO}_{2}{ }^{+}$286.1238; Found 286.1237. $[\alpha]^{28} \mathrm{D}+37.4(c$ 1.0, DCM).

SFC: OD-H column, $5 \% \mathrm{MeOH}$ in supercritical $\mathrm{CO}_{2}$ as eluent, $4 \mathrm{~mL} / \mathrm{min}$. $\mathrm{tR}=5.80 \mathrm{~min}$ (minor), $6.71 \mathrm{~min}$ (major).

(S)-4-(4-chlorophenyl)-3-phenylmorpholin-2-one (1p)

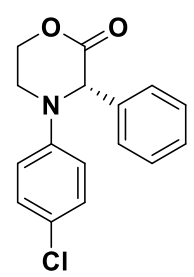

Reaction time: $48 \mathrm{~h}$.

$18.6 \mathrm{mg}$, white foam, $65 \%$ yield, $91 \%$ ee.

${ }^{1}$ H NMR (400 MHz, Chloroform- $d$ ) $\delta 7.48$ - 7.45 (m, 2H), 7.42 - 7.33 (m, 3H), 7.22 - 7.16 (m, 2H), $6.59-6.54(\mathrm{~m}, 2 \mathrm{H}), 5.50(\mathrm{~s}, 1 \mathrm{H}), 4.50-4.42(\mathrm{~m}, 2 \mathrm{H}), 3.75-3.68(\mathrm{~m}, 2 \mathrm{H})$.

${ }^{13}$ C NMR (101 MHz, Chloroform- $d$ ) $\delta$ 168.2, 145.7, 135.6, 129.5, 129.5, 128.8, 126.1, 124.0, 113.4, 64.4, 63.7, 43.6.

IR $\left(v_{\max }, \mathrm{cm}^{-1}\right) 2155,1752,1597,1495,1468,1381,1354,1323,1272,1079,984,809,734$.

HRMS (ESI/QTOF) m/z: [M + H] ${ }^{+}$Calcd for $\mathrm{C}_{16} \mathrm{H}_{15} \mathrm{ClNO}_{2}{ }^{+}$288.0786; Found 288.0777. $[\alpha]^{28} \mathrm{D}+39.7$ (c 1.0, DCM).

SFC: AD-H column, $20 \% \mathrm{MeOH}$ in supercritical $\mathrm{CO}_{2}$ as eluent, $4 \mathrm{~mL} / \mathrm{min}$. $\mathrm{tR}=6.50 \mathrm{~min}$ (major), $7.38 \mathrm{~min}$ (minor).

\section{(S)-4-(2-methoxyphenyl)-3-phenylmorpholin-2-one (1q)}

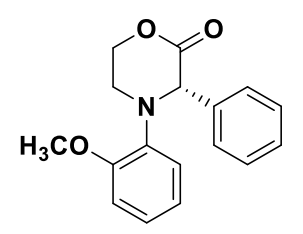

Reaction time: $16 \mathrm{~h}$.

$23.3 \mathrm{mg}$, colorless oil, $82 \%$ yield, $86 \%$ ee.

${ }^{1}$ H NMR (400 MHz, Methylene Chloride- $\left.d_{2}\right) \delta 7.36$ - $7.32(\mathrm{~m}, 2 \mathrm{H}), 7.30$ - 7.19 (m, 3H), 7.01 (ddd, $J=8.1,7.2,1.8 \mathrm{~Hz}, 1 \mathrm{H}), 6.88(\mathrm{dd}, J=8.2,1.4 \mathrm{~Hz}, 1 \mathrm{H}), 6.82(\mathrm{dd}, J=7.8,1.8 \mathrm{~Hz}, 1 \mathrm{H}), 6.75(\mathrm{td}, J=$ 7.6, $1.4 \mathrm{~Hz}, 1 \mathrm{H}), 5.43$ (s, 1H), 4.61 (ddd, $J=11.1,7.8,3.4 \mathrm{~Hz}, 1 \mathrm{H}), 4.48$ (ddd, $J=11.0,5.6,3.6 \mathrm{~Hz}$, $1 \mathrm{H}), 3.86(\mathrm{~s}, 3 \mathrm{H}), 3.57$ (ddd, $J=13.0,5.6,3.4 \mathrm{~Hz}, 1 \mathrm{H}), 3.37$ (ddd, $J=13.0,7.8,3.6 \mathrm{~Hz}, 1 \mathrm{H})$. 
${ }^{13}$ C NMR (101 MHz, Chloroform-d) $\delta$ 169.6, 154.0, 137.4, 137.1, 128.5, 128.0, 127.9, 125.2, 123.7, $121.1,111.9,68.1,65.8,55.5,45.8$.

IR $\left(v_{\max }, \mathrm{cm}^{-1}\right) 2832,2161,2019,1743,1585,1504,1454,1209,1083,1020,987,746,692$.

HRMS (ESI/QTOF) m/z: [M + H] ${ }^{+}$Calcd for $\mathrm{C}_{17} \mathrm{H}_{18} \mathrm{NO}_{3}{ }^{+} 284.1281$; Found 284.1281. $[\alpha]^{26}+114.7$ (c 1.0, DCM).

SFC: AD column, $5 \% \mathrm{MeOH}$ in supercritical $\mathrm{CO}_{2}$ as eluent, $4 \mathrm{~mL} / \mathrm{min} . \mathrm{tR}=6.97 \mathrm{~min}$ (major), 8.82 $\min (\operatorname{minor})$.

(S)-3-(4-fluorophenyl)-4-(2-methoxyphenyl)morpholin-2-one (1r)

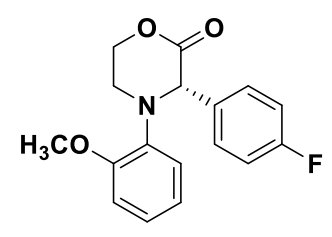

Reaction time: $24 \mathrm{~h}$.

$22.0 \mathrm{mg}$, white foam, $73 \%$ yield, $87 \%$ ee.

${ }^{1}$ H NMR (400 MHz, Chloroform- $d$ ) $\delta 7.38-7.31(\mathrm{~m}, 2 \mathrm{H}), 7.05(\mathrm{ddd}, J=8.2,7.0,2.1 \mathrm{~Hz}, 1 \mathrm{H}), 6.99$ - $6.91(\mathrm{~m}, 2 \mathrm{H}), 6.89(\mathrm{dd}, J=8.2,1.3 \mathrm{~Hz}, 1 \mathrm{H}), 6.85$ - $6.77(\mathrm{~m}, 2 \mathrm{H}), 5.41(\mathrm{~s}, 1 \mathrm{H}), 4.58$ (ddd, $J=11.0$, 7.5, $3.4 \mathrm{~Hz}, 1 \mathrm{H}), 4.51$ (ddd, $J=11.0,5.8,3.8 \mathrm{~Hz}, 1 \mathrm{H}), 3.87$ (s, 3H), 3.56 (ddd, $J=13.0,5.7,3.4 \mathrm{~Hz}$, $1 \mathrm{H}), 3.39$ (ddd, $J=12.9,7.5,3.8 \mathrm{~Hz}, 1 \mathrm{H}$ ).

${ }^{13}$ C NMR (101 MHz, Chloroform- $d$ ) $\delta$ 169.4, 162.5 (d, $J=246.5$ Hz), 154.2, 137.1, 133.0 (d, $J=3.2$ Hz), 129.7 (d, $J=8.2 \mathrm{~Hz}), 125.5,124.0,121.1,115.4$ (d, $J=21.6 \mathrm{~Hz}), 111.9,68.3,65.3,55.5,46.1$.

IR $\left(v_{\max }, \mathrm{cm}^{-1}\right) 2154,2019,1743,1598,1498,1459,1214,1153,1079,1024,815,755$.

HRMS (ESI/QTOF) m/z: [M + H] ${ }^{+}$Calcd for $\mathrm{C}_{17} \mathrm{H}_{17} \mathrm{FNO}_{3}{ }^{+}$302.1187; Found 302.1189. $[\alpha]^{26} \mathrm{D}+74.5$ (c 1.0, DCM).

SFC: AD column, $5 \% \mathrm{MeOH}$ in supercritical $\mathrm{CO}_{2}$ as eluent, $4 \mathrm{~mL} / \mathrm{min}$. $\mathrm{tR}=5.27 \mathrm{~min}$ (major), 6.74 min (minor).

\section{(S)-3-(benzo[d][1,3]dioxol-5-yl)-4-(2,4-dimethoxyphenyl)morpholin-2-one (1s)}<smiles>COc1ccc(N2CCOC(=O)[C@H]2c2ccc3c(c2)OCO3)c(OC)c1</smiles>

Reaction time: $20 \mathrm{~h}$.

$25.7 \mathrm{mg}$, brown oil, $72 \%$ yield, $95 \%$ ee. 
${ }^{1}$ H NMR (400 MHz, Chloroform- $d$ ) $\delta 6.94(\mathrm{~d}, J=1.8 \mathrm{~Hz}, 1 \mathrm{H}), 6.6(\mathrm{~d}, J=8.0,1.6 \mathrm{~Hz}, 1 \mathrm{H}), 6.83(\mathrm{~d}$, $J=8.8 \mathrm{~Hz}, 1 \mathrm{H}), 6.69(\mathrm{~d}, J=8.0 \mathrm{~Hz}, 1 \mathrm{H}), 6.45(\mathrm{~d}, J=2.7 \mathrm{~Hz}, 1 \mathrm{H}), 6.29(\mathrm{dd}, J=8.6,2.7 \mathrm{~Hz}, 1 \mathrm{H})$, $5.90(\mathrm{~s}, 2 \mathrm{H}), 5.16(\mathrm{~s}, 1 \mathrm{H}), 4.61-4.55(\mathrm{~m}, 1 \mathrm{H}), 4.44(\mathrm{dt}, J=10.9,4.0 \mathrm{~Hz}, 1 \mathrm{H}), 3.85(\mathrm{~s}, 3 \mathrm{H}), 3.74(\mathrm{~s}$, $3 \mathrm{H}), 3.42-3.40(\mathrm{~m}, 2 \mathrm{H})$.

${ }^{13}$ C NMR (101 MHz, Chloroform- $d$ ) $\delta$ 170.0, 158.2, 156.2, 147.8, 147.3, 131.6, 130.7, 126.6, 121.7, $108.5,108.1,103.9,101.2,99.9,68.4,66.5,55.6,55.4,47.3$.

IR $\left(v_{\max }, \mathrm{cm}^{-1}\right) 2840,2161,1745,1614,1513,1243,1207,1159,1023,923,804$. $[\alpha]^{26}+127.6(c 1.0, \mathrm{DCM})$.

HRMS (ESI/QTOF) m/z: $[\mathrm{M}+\mathrm{H}]^{+}$Calcd for $\mathrm{C}_{19} \mathrm{H}_{20} \mathrm{NO}_{6}{ }^{+}$358.1285; Found 358.1289.

SFC: IA column, $20 \% \mathrm{MeOH}$ in supercritical $\mathrm{CO}_{2}$ as eluent, $4 \mathrm{~mL} / \mathrm{min}$. $\mathrm{tR}=2.90 \mathrm{~min}$ (major), 4.12 $\min (\operatorname{minor})$.

(S)-4-(2,4-dimethoxyphenyl)-3-phenylmorpholin-2-one (1t)

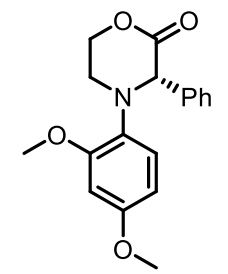

Reaction time: $24 \mathrm{~h}$.

$22.3 \mathrm{mg}$, brown oil, $71 \%$ yield, $86 \%$ ee.

${ }^{1} \mathbf{H}$ NMR (400 MHz, Chloroform- $\left.d\right) \delta 7.41(\mathrm{~d}, J=7.4 \mathrm{~Hz}, 2 \mathrm{H}), 7.31-7.20(\mathrm{~m}, 3 \mathrm{H}), 6.82(\mathrm{~d}, J=8.6$ $\mathrm{Hz}, 1 \mathrm{H}), 6.47(\mathrm{~d}, J=2.7 \mathrm{~Hz}, 1 \mathrm{H}), 6.29(\mathrm{dd}, J=8.6,2.7 \mathrm{~Hz}, 1 \mathrm{H}), 5.26(\mathrm{~s}, 1 \mathrm{H}), 4.57$ (ddd, $J=11.5$, 7.7, $4.0 \mathrm{~Hz}, 1 \mathrm{H})$, , $4.47(\mathrm{dt}, J=11.0,4.3 \mathrm{~Hz}, 1 \mathrm{H}), 3.86(\mathrm{~s}, 3 \mathrm{H}), 3.75(\mathrm{~s}, 3 \mathrm{H}), 3.49-3.37(\mathrm{~m}, 2 \mathrm{H})$.

${ }^{13}$ C NMR (101 MHz, Chloroform- $d$ ) $\delta 169.9,158.2,156.1,137.6,130.9,128.4,128.1,127.9,126.5$, $103.9,100.0,68.3,66.8,55.6,55.4,47.0$.

IR $\left(v_{\max }, \mathrm{cm}^{-1}\right) 2832,1733,1610,1508,1465,1201,1159,1037,827,757,688$.

HRMS (ESI/QTOF) m/z: [M + H] ${ }^{+}$Calcd for $\mathrm{C}_{18} \mathrm{H}_{20} \mathrm{NO}_{4}{ }^{+}$314.1387; Found 314.1388. $[\alpha]^{26}{ }_{\mathrm{D}}+68.9(c$ 1.0, DCM).

SFC: $\mathrm{AD}-\mathrm{H}$ column, $10 \% \mathrm{MeOH}$ in supercritical $\mathrm{CO}_{2}$ as eluent, $4 \mathrm{~mL} / \mathrm{min}$. $\mathrm{tR}=4.77 \mathrm{~min}$ (major), $6.36 \mathrm{~min}$ (minor).

4-(2,4-dimethoxyphenyl)-3-(4-fluorophenyl)morpholin-2-one (1u) 


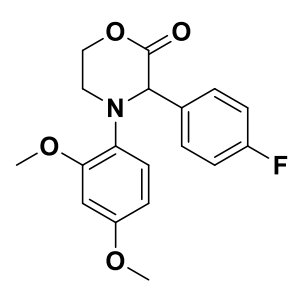

Reaction time: $16 \mathrm{~h}$.

$23.5 \mathrm{mg}$, white foam, $71 \%$ yield, $88 \%$ ee.

${ }^{1}$ H NMR (400 MHz, Chloroform- $d$ ) $\delta 7.37$ (dd, $\left.J=8.5,5.4 \mathrm{~Hz}, 2 \mathrm{H}\right), 6.95$ (t, $\left.J=8.7 \mathrm{~Hz}, 2 \mathrm{H}\right), 6.79$ (d, $J=8.6 \mathrm{~Hz}, 1 \mathrm{H}), 6.45(\mathrm{~d}, J=2.7 \mathrm{~Hz}, 1 \mathrm{H}), 6.28(\mathrm{dd}, J=8.6,2.7 \mathrm{~Hz}, 1 \mathrm{H}), 5.23(\mathrm{~s}, 1 \mathrm{H}), 4.58(\mathrm{ddd}, J=$ 10.9, 7.9, 4.0 Hz, 1H), 4.47 (dt, $J=11.0,4.1 \mathrm{~Hz}, 1 \mathrm{H}), 3.85$ (s, 3H), 3.74 (s, 3H), $3.48-3.35$ (m, 2H). ${ }^{13}$ C NMR (101 MHz, Chloroform- $d$ ) $\delta$ 169.7, $162.5(\mathrm{~d}, J=246.3 \mathrm{~Hz}), 158.4,156.2,133.5(\mathrm{~d}, J=3.2$ $\mathrm{Hz}), 130.5,129.9$ (d, $J=8.2 \mathrm{~Hz}), 126.7,115.3(\mathrm{~d}, J=21.5 \mathrm{~Hz}), 103.9,99.9,68.5,66.2,55.6,55.4$, 47.3.

${ }^{19}$ F NMR (377 MHz, Chloroform-d) $\delta$-114.60.

$[\alpha]^{26}+143.9(c 1.0, \mathrm{DCM})$.

HRMS (nanochip-ESI/LTQ-Orbitrap) m/z: $[\mathrm{M}+\mathrm{H}]^{+}$Calcd for $\mathrm{C}_{18} \mathrm{H}_{19} \mathrm{FNO}_{4}{ }^{+}$332.1293; Found 332.1300 .

SFC: OD-H column, $10 \% \mathrm{MeOH}$ in supercritical $\mathrm{CO}_{2}$ as eluent, $4 \mathrm{~mL} / \mathrm{min}$. $\mathrm{tR}=3.46 \mathrm{~min}$ (minor), $3.9 \min$ (major).

(S)-4-(2,4-dimethoxyphenyl)-3-(4-(trifluoromethyl)phenyl)morpholin-2-one (1v)

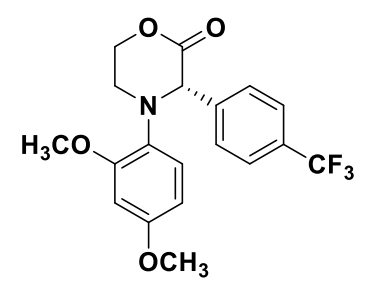

Reaction time: $24 \mathrm{~h}$.

$22.1 \mathrm{mg}$, pale yellow oil, $58 \%$ yield, $87 \%$ ee.

${ }^{1}$ H NMR (400 MHz, Chloroform- $d$ ) $\delta 7.55$ (q, $\left.J=8.3 \mathrm{~Hz}, 4 \mathrm{H}\right), 6.84(\mathrm{~d}, J=8.6 \mathrm{~Hz}, 1 \mathrm{H}), 6.46(\mathrm{~d}, J=$ $2.7 \mathrm{~Hz}, 1 \mathrm{H}), 6.29(\mathrm{dd}, J=8.6,2.7 \mathrm{~Hz}, 1 \mathrm{H}), 5.29$ (s, 1H), $4.58(\mathrm{ddd}, J=11.0,8.8,3.5 \mathrm{~Hz}, 1 \mathrm{H}), 4.47$ $(\mathrm{dt}, J=11.0,3.9 \mathrm{~Hz}, 1 \mathrm{H}), 3.85(\mathrm{~s}, 3 \mathrm{H}), 3.74(\mathrm{~s}, 3 \mathrm{H}), 3.52-3.37(\mathrm{~m}, 2 \mathrm{H})$.

${ }^{13}$ C NMR (101 MHz, Chloroform- $d$ ) $\delta$ 168.1, 157.4, 155.2, 140.7 (q, $\left.J=1.5 \mathrm{~Hz}\right), 129.0$ (q, $J=33.6$ $\mathrm{Hz}), 129.1,127.4,125.8,124.3$ (q, $J=3.8 \mathrm{~Hz}), 123.0$ (q, $J=273.0 \mathrm{~Hz}), 102.9$, 98.9, 67.3, 65.5, 54.4, $54.3,46.5$.

IR $\left(v_{\max }, \mathrm{cm}^{-1}\right)$ 2970, 2016, 1745, 1618, 1519, 1381, 1275, 1210, 1044, 974, 875, 797. 
HRMS (ESI/QTOF) m/z: [M + H] ${ }^{+}$Calcd for $\mathrm{C}_{19} \mathrm{H}_{19} \mathrm{~F}_{3} \mathrm{NO}_{4}{ }^{+} 382.1261$; Found 382.1262. $[\alpha]^{26}+101.7$ (c 1.0, DCM).

SFC: IA column, $3 \% \mathrm{MeOH}$ in supercritical $\mathrm{CO}_{2}$ as eluent, $4 \mathrm{~mL} / \mathrm{min}$. $\mathrm{tR}=6.02 \mathrm{~min}$ (minor), 6.90 $\min$ (major)

\section{(S)-3-([1,1'-biphenyl]-4-yl)-4-(3,4-dimethoxyphenyl)morpholin-2-one (1w)}

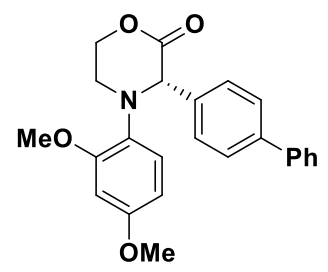

Reaction time: $20 \mathrm{~h}$.

$29.2 \mathrm{mg}$, brown oil, $75 \%$ yield, $85 \%$ ee.

${ }^{1} \mathbf{H}$ NMR $(400 \mathrm{MHz}$, Chloroform- $d) \delta 7.55(\mathrm{~d}, J=8.0 \mathrm{~Hz}, 2 \mathrm{H}), 7.52-7.45(\mathrm{~m}, 4 \mathrm{H}), 7.41(\mathrm{t}, J=7.6$ $\mathrm{Hz}, 2 \mathrm{H}), 7.35-7.30(\mathrm{~m}, 1 \mathrm{H}), 6.86(\mathrm{~d}, J=8.6 \mathrm{~Hz}, 1 \mathrm{H}), 6.47$ (d, $J=2.7 \mathrm{~Hz}, 1 \mathrm{H}), 6.30(\mathrm{dd}, J=8.6,2.5$ $\mathrm{Hz}, 1 \mathrm{H}), 5.30(\mathrm{~s}, 1 \mathrm{H}), 4.64-4.55(\mathrm{~m}, 1 \mathrm{H}), 4.52-4.44(\mathrm{~m}, 1 \mathrm{H}), 3.87(\mathrm{~s}, 3 \mathrm{H}), 3.75$ (s, 3H), $3.51-$ $3.39(\mathrm{~m}, 1 \mathrm{H})$.

${ }^{13}$ C NMR (101 MHz, Chloroform- $d$ ) $\delta$ 170.0, 158.2, 156.1, 140.8, 140.8, 136.7, 130.9, 128.9, 128.5, $127.4,127.20,127.19,126.6,104.0,100.0,68.4,66.6,55.6,55.5,47.1$.

IR $\left(v_{\max }, \mathrm{cm}^{-1}\right) 2832,1733,1610,1508,1465,1201,1159,1037,827,757,688$. $[\alpha]^{26}+165.6$ (c 1.0, DCM).

HRMS (ESI/QTOF) m/z: [M + H] ${ }^{+}$Calcd for $\mathrm{C}_{24} \mathrm{H}_{24} \mathrm{NO}_{4}{ }^{+}$390.1700; Found 390.1702.

SFC: OD-H column, $10 \% \mathrm{MeOH}$ in supercritical $\mathrm{CO}_{2}$ as eluent, $4 \mathrm{~mL} / \mathrm{min}$. $\mathrm{tR}=11.69 \mathrm{~min}$ (minor), $13.12 \mathrm{~min}$ (major).

$(3 S, 6 S)-6-m e t h y l-3-p h e n y l-4-(0-t o l y l) m o r p h o l i n-2-o n e ~(1 x)$

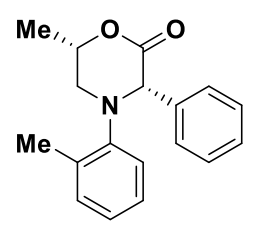

Reaction time: $16 \mathrm{~h}$.

$21.8 \mathrm{mg}$, colorless oil, $78 \%$ yield, $6: 1 \mathrm{dr}$.

${ }^{1}$ H NMR (400 MHz, Chloroform-d) $\delta 7.29$ - 7.24 (m, 3H), 7.23 - 7.17 (m, 3H), 7.12 - 7.06 (m, 1H), $7.06-7.00(\mathrm{~m}, 1 \mathrm{H}), 6.82(\mathrm{~d}, J=7.7 \mathrm{~Hz}, 1 \mathrm{H}), 5.10(\mathrm{~s}, 1 \mathrm{H}), 4.82-4.73(\mathrm{~m}, 1 \mathrm{H}), 3.20(\mathrm{dd}, J=$ 13.7, 8.5 Hz, 1H), $3.08(\mathrm{dd}, J=13.6,3.3 \mathrm{~Hz}, 1 \mathrm{H}), 2.37(\mathrm{~s}, 3 \mathrm{H}), 1.50(\mathrm{~d}, J=6.4 \mathrm{~Hz}, 3 \mathrm{H})$. 
${ }^{13}$ C NMR (101 MHz, Chloroform- $d$ ) $\delta$ 169.8, 147.3, 135.9, 133.4, 131.6, 128.5, 128.4, 128.2, 126.8, $124.9,122.2,75.5,65.7,50.9,19.4,18.4$.

IR $\left(v_{\max }, \mathrm{cm}^{-1}\right)$ 3330, 2976, 2363, 2340, 2018, 1802, 1734, 1440, 1379, 1084, 1047, 877.

HRMS (ESI/QTOF) m/z: [M + H] ${ }^{+}$Calcd for $\mathrm{C}_{18} \mathrm{H}_{20} \mathrm{NO}_{2}{ }^{+} 282.1489$; Found 282.1497.

\section{(3S,6R)-6-methyl-3-phenyl-4-(o-tolyl)morpholin-2-one (1y)}

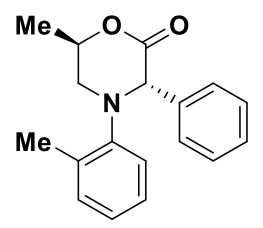

Reaction time: $16 \mathrm{~h}$.

$23.5 \mathrm{mg}$, colorless oil, $84 \%$ yield, > 20: $1 \mathrm{dr}$.

${ }^{1}$ H NMR (400 MHz, Chloroform-d) $\delta 7.36$ - 7.32 (m, 2H), 7.28 - 7.17 (m, 3H), $7.14-7.07$ (m, 3H), $7.01-6.96(\mathrm{~m}, 1 \mathrm{H}), 5.12(\mathrm{~s}, 1 \mathrm{H}), 4.99-4.91(\mathrm{~m}, 1 \mathrm{H}), 3.34(\mathrm{dd}, J=13.0,3.1 \mathrm{~Hz}, 1 \mathrm{H}), 2.95(\mathrm{dd}, J=$ 13.0, $10.0 \mathrm{~Hz}, 1 \mathrm{H}), 2.30$ (s, 3H), 1.43 (d, $J=6.4 \mathrm{~Hz}, 3 \mathrm{H})$.

${ }^{13}$ C NMR (101 MHz, Chloroform- $d$ ) $\delta$ 169.9, 147.8, 137.1, 134.6, 131.4, 128.51, 128.50, 128.2, $126.8,125.3,121.9,75.7,67.3,56.7,19.1,18.2$.

IR $\left(v_{\max }, \mathrm{cm}^{-1}\right)$ 3326, 2977, 2362, 2339, 2019, 1803, 1733, 1442, 1378, 1083, 1045, 875.

HRMS (ESI/QTOF) m/z: [M + H] ${ }^{+}$Calcd for $\mathrm{C}_{18} \mathrm{H}_{20} \mathrm{NO}_{2}{ }^{+}$282.1489; Found 282.1495. $[\alpha]^{26} \mathrm{D}+93.2(c 1.0, \mathrm{DCM})$.

\section{Phenyl(3-(o-tolyl)oxazolidin-2-yl)methanone (4b)}

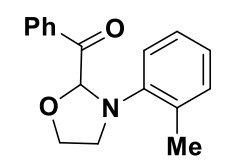

$20.2 \mathrm{mg}$, colorless oil, $75 \%$ yield.

${ }^{1}$ H NMR (400 MHz, Chloroform- $d$ ) $\delta 7.93-7.89(\mathrm{~m}, 2 \mathrm{H}), 7.53(\mathrm{t}, J=7.4 \mathrm{~Hz}, 1 \mathrm{H}), 7.40(\mathrm{t}, J=8.0$ $\mathrm{Hz}, 2 \mathrm{H}), 7.33-7.29(\mathrm{~m}, 1 \mathrm{H}), 7.26-7.20(\mathrm{~m}, 2 \mathrm{H}), 7.11(\mathrm{td}, J=7.3,1.3 \mathrm{~Hz}, 1 \mathrm{H}), 6.22(\mathrm{~s}, 1 \mathrm{H}), 4.26$ $(\mathrm{td}, J=7.5,2.7 \mathrm{~Hz}, 1 \mathrm{H}), 4.01(\mathrm{dt}, J=9.2,7.0 \mathrm{~Hz}, 1 \mathrm{H}), 3.57-3.47(\mathrm{~m}, 1 \mathrm{H}), 3.18(\mathrm{ddd}, J=11.5,6.8$, $2.7 \mathrm{~Hz}, 1 \mathrm{H}), 2.25$ (s, 3H).

${ }^{13}$ C NMR (101 MHz, Chloroform- $d$ ) $\delta$ 194.2, 149.2, 134.7, 134.1, 133.3, 131.3, 129.0, 128.6, 127.2, 125.1, 121.4, 93.4, 65.1, 52.3, 18.5.

IR $\left(v_{\max }, \mathrm{cm}^{-1}\right) 2958,2877,2157,1702,1598,1492,14501315,1224,1105,1051,998,960,885$, $838,755,719,688$. 
HRMS (ESI/QTOF) m/z: [M + H] ${ }^{+}$Calcd for $\mathrm{C}_{17} \mathrm{H}_{18} \mathrm{NO}_{2}{ }^{+}$268.1332; Found 268.1329.

\section{Synthetic transformations}

A solution of $1 \mathrm{t}(180 \mathrm{mg}, 0.6 \mathrm{mmol})$ in dry THF $(10 \mathrm{~mL})$ at $-78^{\circ} \mathrm{C}$ was treated with L-Selectride $(1.0$ M solution in THF, $0.6 \mathrm{~mL}, 0.6 \mathrm{mmol}$ ) in THF. The resulting mixture was stirred for $30 \mathrm{~min}$, then was treated with freshly prepared $8(0.96 \mathrm{mmol}, 1.6$ equiv $)$ in $10 \mathrm{~mL}$ of hexane solution, maintaining the internal temperature below $-60{ }^{\circ} \mathrm{C}$. The reaction mixture was stirred at $-75^{\circ} \mathrm{C}$ for $1 \mathrm{~h}$ and then at $-40^{\circ} \mathrm{C}$ for $1 \mathrm{~h}$. The cold solution was partitioned between $25 \mathrm{~mL}$ of EtOAc and $25 \mathrm{~mL}$ of saturated $\mathrm{NaHCO}_{3}$ and the layers were separated. The aqueous layer was extracted with 2 x $30 \mathrm{~mL}$ of EtOAc. The organic extracts were combined, dried and concentrated in vacuo. Flash chromatography using 20:1 v/v hexanes/EtOAc as the eluant afforded $253 \mathrm{mg}$ (78\%) of 9 as a white foam with dr 11:1.

To a stirred solution of 9 (124.8 mg, $0.4 \mathrm{mmol})$ in $\mathrm{CH}_{3} \mathrm{CN}(12 \mathrm{~mL})$ and $\mathrm{H}_{2} \mathrm{O}(3 \mathrm{~mL})$ at $0{ }^{\circ} \mathrm{C}$ was added CAN (438 mg, $0.8 \mathrm{mmol}$ ) in one portion. Then the resulting solution was stirred at room temperature for $2 \mathrm{~h}$, and quenched with saturated aqueous $\mathrm{NaHCO}_{3}$ solution $(15 \mathrm{~mL})$. The aqueous layer was extracted with $\mathrm{CH}_{2} \mathrm{Cl}_{2}(2 \times 15 \mathrm{~mL})$ and the combined organic layers were washed with $5 \%$ aqueous $\mathrm{Na}_{2} \mathrm{SO}_{3}$ solution $(20 \mathrm{~mL})$, dried over anhydrous $\mathrm{Na}_{2} \mathrm{SO}_{4}$, and concentrated under vacuum. Flash chromatography using 5:1 v/v hexanes/EtOAc as the eluant afforded $60.8 \mathrm{mg}(85 \%)$ of $\mathbf{1 0}$ as a colorless oil.

A mixture of $11(7.3 \mathrm{mg}, 0.055 \mathrm{mmol})$ in DMF $(3 \mathrm{~mL})$ was added dropwise to a cooled $\left(0{ }^{\circ} \mathrm{C}\right)$ stirred mixture of 10 (20.0 mg, $0.05 \mathrm{mmol})$ and powdered $\mathrm{K}_{2} \mathrm{CO}_{3}(7.6 \mathrm{mg}, 0.055 \mathrm{mmol})$ in $\mathrm{DMF} / \mathrm{H}_{2} \mathrm{O}(3 \mathrm{~mL}$, 60:1). After $2 \mathrm{~h}$, water $(20 \mathrm{~mL})$ was added and the aqueous layer was extracted with $\mathrm{CH}_{2} \mathrm{Cl}_{2}(2 \times 15$ $\mathrm{mL})$ and the combined organic layers were washed with brine $(20 \mathrm{~mL})$, dried over anhydrous $\mathrm{Na}_{2} \mathrm{SO}_{4}$, and concentrated under vacuum. Flash chromatography using 40:1:0.1 v/v/v hexanes/EtOAc/Et $3 \mathrm{~N}$ as the eluant afforded $22.9 \mathrm{mg}(91 \%)$ of $7^{[\mathrm{b}]}$ as a white solid.

(2S,3S)-2-((3,5-bis(trifluoromethyl)benzyl)oxy)-4-(2,4-dimethoxyphenyl)-3-phenylmorpholine (9)

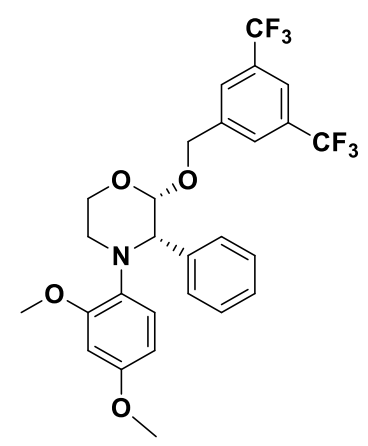

Pale yellow oil, $78 \%$ yield, 11:1 dr. 
${ }^{1}$ H NMR (400 MHz, Chloroform- $\left.d\right) \delta 7.70(\mathrm{~s}, 1 \mathrm{H}), 7.45(\mathrm{~s}, 2 \mathrm{H}), 7.31-7.27(\mathrm{~m}, 2 \mathrm{H}), 7.19-7.10(\mathrm{~m}$, $3 \mathrm{H}), 6.72(\mathrm{~d}, J=8.6 \mathrm{~Hz}, 1 \mathrm{H}), 6.37(\mathrm{~d}, J=2.7 \mathrm{~Hz}, 1 \mathrm{H}), 6.19(\mathrm{dd}, J=8.7,2.8 \mathrm{~Hz}, 1 \mathrm{H}), 4.89-4.84(\mathrm{~m}$, $2 \mathrm{H}), 4.61(\mathrm{~d}, J=3.0 \mathrm{~Hz}, 1 \mathrm{H}), 4.52(\mathrm{~d}, J=13.6 \mathrm{~Hz}, 1 \mathrm{H}), 4.36(\mathrm{td}, J=10.9,2.8 \mathrm{~Hz}, 1 \mathrm{H}), 3.84(\mathrm{~s}, 3 \mathrm{H})$, $3.74(\mathrm{dt}, J=11.2,3.1 \mathrm{~Hz}, 1 \mathrm{H}), 3.68(\mathrm{~s}, 3 \mathrm{H}), 3.29(\mathrm{dt}, J=11.9,2.8 \mathrm{~Hz}, 1 \mathrm{H}), 3.04(\mathrm{td}, J=11.3,3.3 \mathrm{~Hz}$, $1 \mathrm{H})$.

${ }^{13}$ C NMR (101 MHz, Chloroform- $d$ ) $\delta$ 157.2, 156.0, 141.1, 137.5, 132.5, 131.5 (q, $J=33.3 \mathrm{~Hz}$ ), 129.3, 127.7, 127.4, $127.2(\mathrm{q}, J=2.0 \mathrm{~Hz}), 125.3,123.4(\mathrm{q}, J=273.7 \mathrm{~Hz}), 121.3(\mathrm{q}, J=4.0 \mathrm{~Hz})$, 103.6, 99.9, 99.3, 67.8, 66.1, 61.1, 55.5, 55.4, 52.3.

IR $\left(v_{\max }, \mathrm{cm}^{-1}\right)$ 2356, 2325, 2040, 1508, 1454, 1276, 1203, 1164, 1132, 1074, 1037, 906.

HRMS (ESI/QTOF) m/z: [M + H] ${ }^{+}$Calcd for $\mathrm{C}_{27} \mathrm{H}_{26} \mathrm{~F}_{6} \mathrm{NO}_{4}{ }^{+}$542.1761; Found 542.1763. $[\alpha]^{26} \mathrm{D}+105.8(c 1.0, \mathrm{DCM})$.

(2S,3S)-2-((3,5-bis(trifluoromethyl)benzyl)oxy)-3-phenylmorpholine (10)

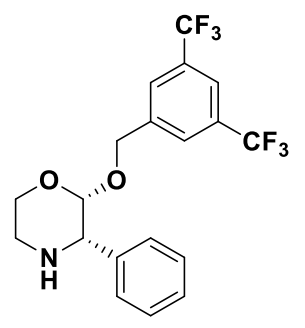

$60.8 \mathrm{mg}$, colorless oil, $85 \%$ yield.

${ }^{1}$ H NMR (400 MHz, Chloroform- $\left.d\right) \delta 7.70(\mathrm{~s}, 1 \mathrm{H}), 7.42(\mathrm{~s}, 2 \mathrm{H}), 7.38-7.28(\mathrm{~m}, 4 \mathrm{H}), \quad 4.81(\mathrm{~d}, J=$ $13.5 \mathrm{~Hz}, 1 \mathrm{H}), 4.76(\mathrm{~d}, J=2.3 \mathrm{~Hz}, 1 \mathrm{H}), 4.51$ (d, $J=13.5 \mathrm{~Hz}, 1 \mathrm{H}), 4.13(\mathrm{~d}, J=2.4 \mathrm{~Hz}, 1 \mathrm{H}), 4.06(\mathrm{td}, J$ $=11.7,3.0 \mathrm{~Hz}, 1 \mathrm{H}), 3.64(\mathrm{dd}, J=11.3,3.6 \mathrm{~Hz}, 1 \mathrm{H}), 3.25(\mathrm{td}, J=12.3,3.6 \mathrm{~Hz}, 1 \mathrm{H}), 3.12(\mathrm{dd}, J=$ 12.7, $3.0 \mathrm{~Hz}, 1 \mathrm{H}), 2.08(\mathrm{~s}, 1 \mathrm{H})$.

${ }^{13}$ C NMR (101 MHz, Chloroform- $d$ ) $\delta$ 140.7, 138.9, 131.6 (q, $\left.J=33.3 \mathrm{~Hz}\right), 128.5,127.9,127.3$ (q, $J$ $=3.0 \mathrm{~Hz}), 127.1,123.3(\mathrm{q}, J=272.8 \mathrm{~Hz}), 121.4(\mathrm{q}, J=4.0 \mathrm{~Hz}), 98.0,67.7,62.8,59.6,46.5$.

${ }^{19}$ F NMR (377 MHz, Chloroform- $d$ ) $\delta-62.8$.

HRMS (ESI/QTOF) m/z: [M + H] ${ }^{+}$Calcd for $\mathrm{C}_{19} \mathrm{H}_{18} \mathrm{~F}_{6} \mathrm{NO}_{2}{ }^{+}$406.1236; Found 406.1234.

IR $\left(v_{\max }, \mathrm{cm}^{-1}\right)$ 2902, 2871, 1963, 1454, 1375, 1276, 1174, 1128, 1062, 885, 838, 719, 698.

$[\alpha]^{23}+103.8\left(c 1.0, \mathrm{CHCl}_{3}\right)$.

5-(((2S,3S)-2-((3,5-bis(trifluoromethyl)benzyl)oxy)-3-phenylmorpholino)methyl)-2,4-dihydro-3 H-1,2,4-triazol-3-one (7, L-724,694) 


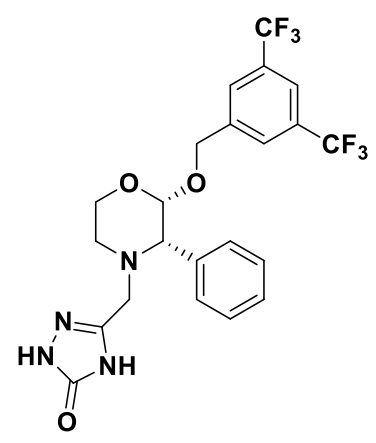

$22.9 \mathrm{mg}$, M.p $=155-157^{\circ} \mathrm{C}$. white solid, $91 \%$ yield.

${ }^{1}$ H NMR (400 MHz, DMSO-d6) $\delta 11.37$ (s, 1H), 11.24 (s, 1H), 7.92 (s, 1H), $7.64(\mathrm{~s}, 2 \mathrm{H}), 7.56$ (d, J $=6.5 \mathrm{~Hz}, 2 \mathrm{H}), 7.36-7.26(\mathrm{~m}, 3 \mathrm{H}), 4.79(\mathrm{~d}, J=14.0 \mathrm{~Hz}, 1 \mathrm{H}), 4.75(\mathrm{~d}, J=2.8 \mathrm{~Hz}, 1 \mathrm{H}), 4.44(\mathrm{~d}, J=$ $14.0 \mathrm{~Hz}, 1 \mathrm{H}), 4.00(\mathrm{td}, J=11.6,2.4 \mathrm{~Hz}, 1 \mathrm{H}), 3.65-3.60(\mathrm{~m}, 1 \mathrm{H}), 3.58(\mathrm{~d}, J=2.8 \mathrm{~Hz}, 1 \mathrm{H}), 3.42(\mathrm{~d}, J$ $=13.9 \mathrm{~Hz}, 1 \mathrm{H}), 2.77(\mathrm{~d}, J=13.8 \mathrm{~Hz}, 2 \mathrm{H}), 2.39(\mathrm{td}, J=11.7,3.5 \mathrm{~Hz}, 1 \mathrm{H})$.

${ }^{13}$ C NMR (101 MHz, DMSO-d6) $\delta$ 156.3, 143.9, 141.9, 137.4, 130.0 (q, $J=32.7$ Hz), 129.3, 127.8, 127.5, $127.2(\mathrm{q}, J=3.0 \mathrm{~Hz}), 123.3(\mathrm{q}, J=272.9 \mathrm{~Hz}), 120.8(\mathrm{q}, J=4.0 \mathrm{~Hz}), 98.0,68.6,66.7,58.8$, $51.3,50.6$.

${ }^{19}$ F NMR $\left(377 \mathrm{MHz}, \mathrm{DMSO}-d_{6}\right) \delta-61.25$.

IR $\left(v_{\max }, \mathrm{cm}^{-1}\right)$ 2366, 1700, 1373, 1278, 1168, 1126, 1056, 1037, 906, 890, 719.

HRMS (ESI/QTOF) m/z: $[\mathrm{M}+\mathrm{H}]^{+}$Calcd for $\mathrm{C}_{22} \mathrm{H}_{21} \mathrm{~F}_{6} \mathrm{~N}_{4} \mathrm{O}_{3}{ }^{+}$503.1512; Found 503.1513. $[\alpha]^{23} \mathrm{D}+60.8\left(c 1.0, \mathrm{CHCl}_{3}\right)$.

\section{(S)-3-(4-fluorophenyl)morpholin-2-one (12)}

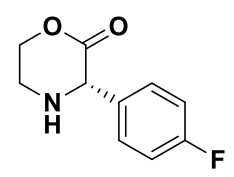

$15.8 \mathrm{mg}$, White foam, $81 \%$ yield.

${ }^{1}$ H NMR (400 MHz, Chloroform-d) $\delta 7.39-7.35$ (m, 2H), $7.05-7.01(\mathrm{~m}, 2 \mathrm{H}), 3.70-3.61(\mathrm{~m}, 2 \mathrm{H})$, $3.48-3.30(\mathrm{~m}, 2 \mathrm{H}), 2.97(\mathrm{~s}, 1 \mathrm{H})$.

${ }^{13}$ C NMR (101 MHz, Chloroform- $d$ ) $\delta$ 173.5, $163.0(\mathrm{~d}, J=247.4 \mathrm{~Hz}), 135.1$ (d, $\left.J=3.2 \mathrm{~Hz}\right), 128.6(\mathrm{~d}$, $J=8.3 \mathrm{~Hz}), 115.9(\mathrm{~d}, J=21.7 \mathrm{~Hz}), 73.8,61.9,42.2$.

${ }^{19}$ F NMR (377 MHz, Chloroform- $d$ ) $\delta$-113.20.

IR $\left(v_{\max }, \mathrm{cm}^{-1}\right)$ 3322, 2368, 2152, 1654, 1540, 1502, 1228, 1074, 833, 804, 719.

HRMS (ESI/QTOF) m/z: [M + H] $]^{+}$Calcd for $\mathrm{C}_{10} \mathrm{H}_{11} \mathrm{FNO}_{2}{ }^{+}$196.0774; Found 196.0772. $[\alpha]^{26} \mathrm{D}+38.6\left(c 1.0, \mathrm{CHCl}_{3}\right)$. 


\section{Reference:}

[a] H. Yin, M. Jin, W. Chen, C. Chen, L. Zheng, P. Wei, S. Han, Tetrahedron Lett. 2012, 53, 1265-1270.

[b] C. J. Cowden, R. D. Wilson, B. C. Bishop, I. F. Cottrell, A. J. Davies, U. H. Dolling, Tetrahedron Lett. 2000, 41, 8661-8664.

\section{Control experiments ${ }^{(a)}$.}<smiles>OCCNc1ccc(F)cc1</smiles>

30

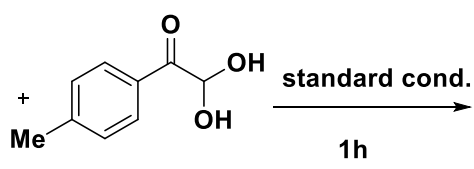

20

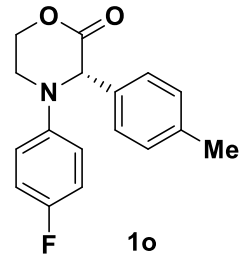

10

\begin{tabular}{|c|c|c|}
\hline Time (h) & Conversion (\%) & Ee (\%) \\
\hline 2 & $10 \%$ & 91 \\
\hline 5 & $20 \%$ & 91 \\
\hline 17 & $36 \%$ & 90 \\
\hline 41 & $71 \%$ & 87 \\
\hline 65 & $80 \%$ & 86 \\
\hline 89 & $100 \%$ & 83 \\
\hline 161 & $100 \%$ & 71 \\
\hline 185 & $100 \%$ & 65 \\
\hline 377 & $100 \%$ & 39 \\
\hline
\end{tabular}

(a) The reaction was performed on $0.1 \mathrm{mmol}$ scale. Enantiomeric excess (ee) was determined by SFC analysis on a chiral stationary phase.

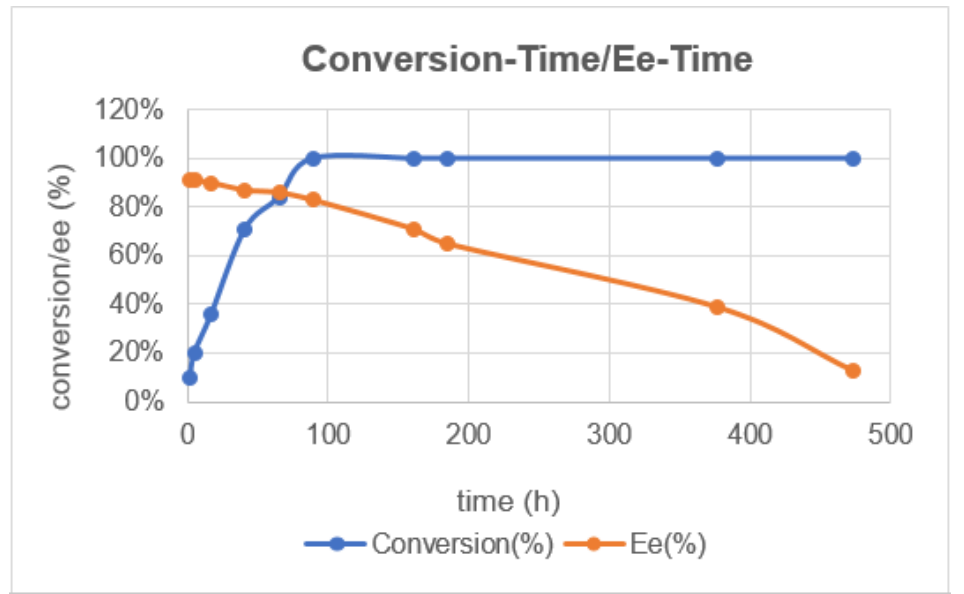




\section{Crystallographic data for $1 \mathrm{~m}$}
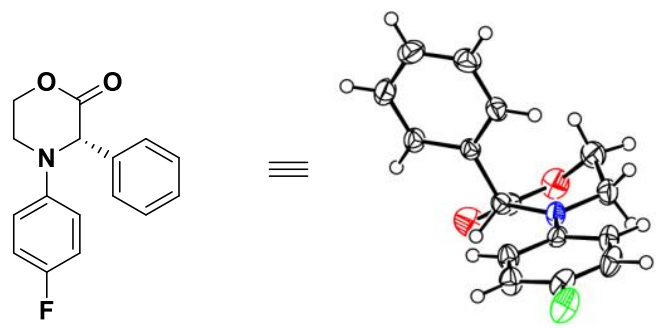

\begin{tabular}{|c|c|}
\hline Compound & YP-6-29 \\
\hline Formula & $\mathrm{C}_{16} \mathrm{H}_{14} \mathrm{FNO}_{2}$ \\
\hline$D_{\text {calc. }} / \mathrm{g} \mathrm{cm}^{-3}$ & 1.383 \\
\hline$\mu / \mathrm{mm}^{-1}$ & 0.837 \\
\hline Formula Weight & 271.28 \\
\hline Colour & colourless \\
\hline Shape & hexagonal \\
\hline Size $/ \mathrm{mm}^{3}$ & $0.18 \times 0.16 \times 0.11$ \\
\hline$T / \mathrm{K}$ & $140.00(10)$ \\
\hline Crystal System & triclinic \\
\hline Flack Parameter & $0.04(3)$ \\
\hline Hooft Parameter & $0.05(3)$ \\
\hline Space Group & $P 1$ \\
\hline$a / \AA ̊$ & $7.82378(14)$ \\
\hline$b / \AA$ & $9.79404(17)$ \\
\hline$c / \AA$ & $17.3653(3)$ \\
\hline$\alpha /^{\circ}$ & $88.0648(14)$ \\
\hline$\beta /^{\circ}$ & $79.0877(15)$ \\
\hline$\gamma /^{\circ}$ & $85.7449(14)$ \\
\hline $\mathrm{V} / \AA^{3}$ & $1302.71(4)$ \\
\hline$Z$ & 4 \\
\hline$Z^{\prime}$ & 4 \\
\hline Wavelength/Å & 1.54184 \\
\hline Radiation type & $\mathrm{CuK \alpha}$ \\
\hline$\Theta_{\min } /^{\circ}$ & 4.528 \\
\hline$\Theta_{\max } /^{\circ}$ & 76.074 \\
\hline Measured Refl's. & 26645 \\
\hline Ind't Refl's & 10100 \\
\hline Refl's with $\mathrm{I}>2 \sigma(\mathrm{I})$ & 9828 \\
\hline$R_{\text {int }}$ & 0.0193 \\
\hline Parameters & 721 \\
\hline Restraints & 3 \\
\hline Largest Peak/e $\AA^{-3}$ & 0.201 \\
\hline Deepest Hole/e $\AA^{-3}$ & -0.186 \\
\hline GooF & 1.026 \\
\hline$w R_{2}$ (all data) & 0.0834 \\
\hline$w R_{2}$ & 0.0822 \\
\hline$R_{1}$ (all data) & 0.0313 \\
\hline$R_{1}$ & 0.0304 \\
\hline
\end{tabular}


Table 1: Fractional Atomic Coordinates $\left(\times 10^{4}\right)$ and Equivalent Isotropic Displacement Parameters $\left(\AA^{2} \times 10^{3}\right)$ for yp-6-29. $U_{e q}$ is defined as $1 / 3$ of the trace of the orthogonalised $U_{i j}$.

\begin{tabular}{|c|c|c|c|c|}
\hline Atom & $\mathbf{x}$ & $\mathbf{y}$ & $\mathbf{z}$ & $U_{e q}$ \\
\hline$\overline{F 1}$ & $6206(2)$ & $10417.2(14)$ & 1763.7(8) & $49.0(4)$ \\
\hline 01 & $8190(2)$ & 3937.1(17) & $-1593.8(9)$ & $39.5(4)$ \\
\hline 02 & $7468(3)$ & $2939.9(16)$ & $-449.7(10)$ & $45.1(4)$ \\
\hline N1 & $7469(2)$ & $5403.0(15)$ & $304.8(8)$ & $24.2(3)$ \\
\hline $\mathrm{C} 1$ & $8336(2)$ & $5335.4(18)$ & $-513.4(10)$ & $22.9(3)$ \\
\hline $\mathrm{C} 2$ & $7964(3)$ & $4023(2)$ & $-893.6(12)$ & $30.1(4)$ \\
\hline $\mathrm{C} 3$ & $6861(4)$ & $3040(2)$ & $389.4(14)$ & $49.0(6)$ \\
\hline $\mathrm{C} 4$ & $7666(3)$ & $4142(2)$ & $748.9(12)$ & $33.1(4)$ \\
\hline $\mathrm{C} 5$ & $10316(2)$ & $5487.6(17)$ & $-678.6(10)$ & $21.8(3)$ \\
\hline $\mathrm{C} 6$ & $11250(3)$ & $5590.9(19)$ & $-76.7(10)$ & $26.3(4)$ \\
\hline $\mathrm{C} 7$ & $13041(3)$ & $5729(2)$ & $-251.0(12)$ & $32.6(4)$ \\
\hline $\mathrm{C} 8$ & $13898(3)$ & $5790(2)$ & $-1025.6(13)$ & $32.6(4)$ \\
\hline $\mathrm{C} 9$ & $12978(3)$ & $5710(2)$ & $-1630.0(11)$ & $28.7(4)$ \\
\hline C10 & $11195(3)$ & $5559.7(18)$ & $-1453.8(10)$ & $25.0(4)$ \\
\hline C11 & $7226(2)$ & $6680.7(19)$ & $672.8(10)$ & $23.0(3)$ \\
\hline C12 & $6646(3)$ & 7843.1(19) & $281.5(11)$ & $29.4(4)$ \\
\hline C13 & $6299(3)$ & $9098(2)$ & $648.3(12)$ & $34.8(4)$ \\
\hline C14 & $6543(3)$ & $9181(2)$ & $1408.8(12)$ & $33.9(4)$ \\
\hline C15 & $7117(3)$ & $8069(2)$ & $1816.3(11)$ & $32.9(4)$ \\
\hline C16 & $7468(3)$ & $6810(2)$ & $1446.9(10)$ & $28.1(4)$ \\
\hline $\mathrm{F} 2$ & $2053(2)$ & $5421.3(15)$ & $6558.1(7)$ & $48.4(4)$ \\
\hline 03 & $3455(2)$ & $3601(2)$ & $1752.3(10)$ & $47.3(4)$ \\
\hline 04 & $3057(2)$ & $5844.7(19)$ & $1700.0(8)$ & $45.8(4)$ \\
\hline $\mathrm{N} 2$ & $2260(2)$ & $5813.9(16)$ & $3354.4(9)$ & $27.5(3)$ \\
\hline C17 & 1931(2) & $4638.5(18)$ & $2931.9(10)$ & $23.0(4)$ \\
\hline C18 & $2890(3)$ & $4642(2)$ & 2082.3(11) & $33.3(4)$ \\
\hline C19 & $2297(4)$ & $7048(3)$ & $2126.4(13)$ & $43.1(5)$ \\
\hline $\mathrm{C} 20$ & $2803(3)$ & $7043(2)$ & $2921.6(11)$ & $32.4(4)$ \\
\hline C21 & $-4(2)$ & $4439.6(18)$ & 2956.3(9) & $21.6(3)$ \\
\hline C22 & $-1297(3)$ & $5351(2)$ & $3349.0(11)$ & $28.4(4)$ \\
\hline C23 & $-3039(3)$ & $5088(3)$ & $3423.6(13)$ & $38.9(5)$ \\
\hline $\mathrm{C} 24$ & $-3494(3)$ & $3917(3)$ & $3111.6(14)$ & $42.9(6)$ \\
\hline $\mathrm{C} 25$ & $-2222(3)$ & $3020(2)$ & $2704.9(13)$ & $38.7(5)$ \\
\hline C26 & $-471(3)$ & $3275.9(19)$ & $2623.0(11)$ & $27.7(4)$ \\
\hline $\mathrm{C} 27$ & $2244(2)$ & $5701.7(19)$ & 4158.3(10) & $24.2(3)$ \\
\hline C28 & $2905(3)$ & $6719.4(19)$ & 4549.1(11) & $27.4(4)$ \\
\hline C29 & $2866(3)$ & $6619(2)$ & $5352.2(12)$ & $31.4(4)$ \\
\hline C30 & $2145(3)$ & $5510(2)$ & $5762.1(11)$ & $33.6(4)$ \\
\hline C31 & $1483(3)$ & $4489(2)$ & $5407.1(12)$ & $36.3(5)$ \\
\hline C32 & $1541(3)$ & $4580(2)$ & $4600.8(11)$ & $31.0(4)$ \\
\hline F3 & $6032(2)$ & $5757.9(14)$ & $6571.8(8)$ & $46.9(4)$ \\
\hline 05 & $7628(3)$ & $-1205.9(17)$ & $3653.5(10)$ & $46.6(4)$ \\
\hline 06 & $7849(2)$ & $-2118.8(15)$ & $4805.5(10)$ & $41.9(4)$ \\
\hline N3 & $7584(2)$ & $532.4(17)$ & $5394.4(9)$ & $28.3(3)$ \\
\hline C33 & $8317(3)$ & $331.6(18)$ & $4572.9(10)$ & $24.6(4)$ \\
\hline C34 & $7898(3)$ & $-1059(2)$ & $4299.6(13)$ & $32.9(4)$ \\
\hline C35 & $8468(3)$ & $-1880(2)$ & $5524.8(13)$ & $39.1(5)$ \\
\hline C36 & $7520(3)$ & $-638(2)$ & $5930.9(12)$ & $34.1(4)$ \\
\hline C37 & $10284(3)$ & $505.0(18)$ & $4339.5(10)$ & $24.2(3)$ \\
\hline C38 & $11315(3)$ & $655(2)$ & $4898.0(11)$ & $30.0(4)$ \\
\hline C39 & $13091(3)$ & $819(2)$ & $4667.3(14)$ & $39.1(5)$ \\
\hline C40 & $13835(3)$ & $845(2)$ & $3873.4(15)$ & $40.5(5)$ \\
\hline C41 & $12812(3)$ & $707(2)$ & $3315.3(12)$ & $36.6(5)$ \\
\hline C42 & $11045(3)$ & $532(2)$ & $3547.4(11)$ & $29.8(4)$ \\
\hline C43 & $7213(3)$ & $1853(2)$ & $5684.6(10)$ & $25.2(4)$ \\
\hline
\end{tabular}




\begin{tabular}{lcccc}
\hline Atom & $\mathbf{x}$ & $\mathbf{y}$ & $\mathbf{z}$ & $\boldsymbol{U}_{\boldsymbol{e q}}$ \\
\hline C44 & $6749(3)$ & $2058(2)$ & $6496.6(11)$ & $33.3(4)$ \\
C45 & $6372(3)$ & $3372(2)$ & $6790.7(11)$ & $37.7(5)$ \\
C46 & $6425(3)$ & $4467(2)$ & $6282.5(12)$ & $32.9(4)$ \\
C47 & $6872(3)$ & $4320(2)$ & $5484.0(12)$ & $35.3(4)$ \\
C48 & $7290(3)$ & $3011(2)$ & $5188.8(11)$ & $31.2(4)$ \\
F4 & $1341(2)$ & $271.5(15)$ & $11631.6(7)$ & $50.3(4)$ \\
O7 & $3029(2)$ & $-1243(2)$ & $6747.8(10)$ & $49.1(5)$ \\
O8 & $2401(3)$ & $981.9(18)$ & $6762.2(8)$ & $45.8(4)$ \\
N4 & $1750(2)$ & $781.9(15)$ & $8420.0(9)$ & $27.1(3)$ \\
C49 & $1478(2)$ & $-373.9(18)$ & $7966.2(10)$ & $23.3(4)$ \\
C50 & $2378(3)$ & $-254(2)$ & $7109.6(11)$ & $33.2(4)$ \\
C51 & $1586(4)$ & $2120(2)$ & $7241.0(13)$ & $43.7(5)$ \\
C52 & $2172(3)$ & $2062(2)$ & $8018.0(12)$ & $31.5(4)$ \\
C53 & $-442(2)$ & $-646.2(18)$ & $8004.9(10)$ & $23.2(3)$ \\
C54 & $-1780(3)$ & $243(2)$ & $8385.6(12)$ & $31.6(4)$ \\
C55 & $-3507(3)$ & $-72(3)$ & $8449.5(15)$ & $44.2(6)$ \\
C56 & $-3900(3)$ & $-1262(3)$ & $8142.6(15)$ & $48.1(6)$ \\
C57 & $-2578(4)$ & $-2145(3)$ & $7758.1(13)$ & $43.2(6)$ \\
C58 & $-842(3)$ & $-1840(2)$ & $7682.4(11)$ & $30.1(4)$ \\
C59 & $1684(2)$ & $635.5(19)$ & $9222.1(10)$ & $23.3(3)$ \\
C60 & $1111(3)$ & $-555.6(19)$ & $9633.8(10)$ & $27.5(4)$ \\
C61 & $1002(3)$ & $-681(2)$ & $10440.6(11)$ & $31.8(4)$ \\
C62 & $1470(3)$ & $388(2)$ & $10833.6(11)$ & $33.6(4)$ \\
C63 & $2066(3)$ & $1562(2)$ & $10458.6(12)$ & $32.6(4)$ \\
C64 & $2176(3)$ & $1689.7(19)$ & $9649.2(11)$ & $27.4(4)$ \\
& & & &
\end{tabular}

Table 2: Anisotropic Displacement Parameters $\left(\times 10^{4}\right)$ for yp-6-29. The anisotropic displacement factor exponent takes the form: $-2 \pi^{2}\left[h^{2} a^{* 2} \times U_{11}+\ldots+2 h k a^{*} \times b^{*} \times U_{12}\right]$

\begin{tabular}{|c|c|c|c|c|c|c|}
\hline Atom & $U_{11}$ & $U_{22}$ & $U_{33}$ & $U_{23}$ & $U_{13}$ & $U_{12}$ \\
\hline F1 & $64.0(9)$ & $33.5(7)$ & $44.5(7)$ & $-19.4(5)$ & $8.9(6)$ & $-10.0(6)$ \\
\hline 01 & $38.7(8)$ & $47.9(9)$ & $33.8(7)$ & $-16.7(6)$ & $-6.6(6)$ & $-8.9(7)$ \\
\hline 02 & $58.1(11)$ & $30.9(7)$ & $42.3(8)$ & $-13.5(6)$ & $7.5(7)$ & $-13.6(7)$ \\
\hline N1 & $27.2(8)$ & $22.7(7)$ & $21.1(7)$ & $-1.9(6)$ & $-0.5(6)$ & $-1.6(6)$ \\
\hline C1 & $23.4(9)$ & $24.9(8)$ & $20.4(8)$ & $-3.9(6)$ & $-3.2(6)$ & $-2.3(7)$ \\
\hline $\mathrm{C} 2$ & $22.4(9)$ & $33.8(10)$ & $34.2(10)$ & $-11.3(8)$ & $-2.7(7)$ & $-4.0(8)$ \\
\hline C3 & $66.9(17)$ & $34.8(11)$ & $40.1(12)$ & $-5.8(9)$ & $10.7(11)$ & $-20.7(11)$ \\
\hline $\mathrm{C} 4$ & $42.6(12)$ & $25.0(9)$ & $29.0(9)$ & $2.5(7)$ & $0.4(8)$ & $-4.3(8)$ \\
\hline $\mathrm{C} 5$ & $24.2(9)$ & $19.7(8)$ & $21.4(7)$ & $-0.5(6)$ & $-4.1(6)$ & $-0.7(7)$ \\
\hline $\mathrm{C} 6$ & 28.1(10) & 29.6(9) & $21.5(8)$ & $-0.3(6)$ & $-5.7(7)$ & $-2.0(7)$ \\
\hline C7 & $28.8(10)$ & $39.8(11)$ & $32.0(9)$ & $-0.2(8)$ & $-11.6(8)$ & $-4.4(8)$ \\
\hline C8 & $21.6(9)$ & $36.5(10)$ & $38.9(10)$ & $1.3(8)$ & $-3.5(8)$ & $-4.3(8)$ \\
\hline C9 & $28.6(10)$ & $29.6(9)$ & $25.8(8)$ & $0.2(7)$ & $0.4(7)$ & $-2.9(7)$ \\
\hline C10 & $28.0(9)$ & $25.2(8)$ & $21.9(8)$ & $-1.8(6)$ & $-5.2(7)$ & $-1.8(7)$ \\
\hline C11 & $21.9(8)$ & $24.9(8)$ & $21.8(8)$ & $-3.6(6)$ & $-1.1(6)$ & $-3.9(7)$ \\
\hline C12 & $35.7(10)$ & $26.8(9)$ & $25.7(8)$ & $-2.9(7)$ & $-5.6(7)$ & $-2.1(8)$ \\
\hline C13 & $40.5(12)$ & $26.2(9)$ & $36.2(10)$ & $-2.8(8)$ & $-3.8(9)$ & $-0.6(8)$ \\
\hline C14 & $35.8(11)$ & $28.6(9)$ & $33.8(10)$ & $-12.3(8)$ & $6.9(8)$ & $-7.9(8)$ \\
\hline C15 & $36.4(11)$ & $39.8(11)$ & $21.9(8)$ & $-7.1(7)$ & $1.4(8)$ & $-12.8(9)$ \\
\hline C16 & $30.8(10)$ & $32.1(9)$ & $20.8(8)$ & $-0.1(7)$ & $-2.1(7)$ & $-6.1(8)$ \\
\hline F2 & $73.3(11)$ & $54.3(8)$ & $21.0(6)$ & $-4.3(5)$ & $-17.1(6)$ & $-3.8(7)$ \\
\hline 03 & $39.0(9)$ & $64.8(11)$ & $35.6(8)$ & $-25.5(8)$ & $4.8(7)$ & $-7.5(8)$ \\
\hline 04 & $57.3(11)$ & $60.3(10)$ & $20.7(6)$ & $2.3(6)$ & $-2.7(7)$ & $-24.1(9)$ \\
\hline $\mathrm{N} 2$ & $35.8(9)$ & $27.5(8)$ & $21.3(7)$ & $0.0(6)$ & $-7.5(6)$ & $-10.8(7)$ \\
\hline C17 & $24.5(9)$ & $25.9(9)$ & $18.9(7)$ & $-4.0(6)$ & $-4.7(7)$ & $-1.4(7)$ \\
\hline C18 & $26.2(10)$ & $50.2(12)$ & $23.9(8)$ & $-8.1(8)$ & $-1.6(7)$ & $-10.7(9)$ \\
\hline C19 & $54.0(14)$ & $46.4(12)$ & $32.4(10)$ & $13.6(9)$ & $-15.7(10)$ & $-14.2(11)$ \\
\hline
\end{tabular}




\begin{tabular}{|c|c|c|c|c|c|c|}
\hline Atom & $U_{11}$ & $U_{22}$ & $U_{33}$ & $\boldsymbol{U}_{23}$ & $U_{13}$ & $U_{12}$ \\
\hline$\overline{\mathrm{C} 20}$ & $41.3(12)$ & $30.0(9)$ & $27.7(9)$ & $4.7(7)$ & $-8.8(8)$ & $-10.4(9)$ \\
\hline $\mathrm{C} 21$ & $22.0(9)$ & $24.8(8)$ & $18.0(7)$ & $2.0(6)$ & $-4.1(6)$ & $-0.7(7)$ \\
\hline $\mathrm{C} 22$ & $29.5(10)$ & $31.0(9)$ & $23.2(8)$ & $0.3(7)$ & $-3.5(7)$ & $3.5(8)$ \\
\hline C23 & $27.1(11)$ & $52.7(13)$ & $32.6(10)$ & $9.9(9)$ & $-0.8(8)$ & $7.8(9)$ \\
\hline $\mathrm{C} 24$ & $23.7(10)$ & $65.5(15)$ & $41.0(11)$ & $20.2(11)$ & $-11.6(9)$ & $-8.9(10)$ \\
\hline $\mathrm{C} 25$ & $39.2(13)$ & $44.0(12)$ & $38.9(11)$ & $9.2(9)$ & $-19.1(9)$ & $-15.8(10)$ \\
\hline $\mathrm{C} 26$ & $30.3(10)$ & $28.2(9)$ & $26.4(8)$ & $-0.8(7)$ & $-8.9(7)$ & $-3.3(8)$ \\
\hline $\mathrm{C} 27$ & $25.1(9)$ & $26.6(8)$ & $21.4(8)$ & $-4.0(6)$ & $-5.3(7)$ & $-1.2(7)$ \\
\hline C28 & $28.7(9)$ & $26.3(8)$ & $27.5(9)$ & $-5.4(7)$ & $-5.2(7)$ & $-1.7(7)$ \\
\hline C29 & $33.3(10)$ & $32.6(10)$ & $30.3(9)$ & $-12.1(7)$ & $-10.5(8)$ & $1.9(8)$ \\
\hline C30 & $40.8(12)$ & $42.1(11)$ & $19.3(8)$ & $-4.2(7)$ & $-10.9(8)$ & $3.7(9)$ \\
\hline C31 & $48.0(13)$ & $38.0(11)$ & $25.0(9)$ & $3.8(8)$ & $-11.3(9)$ & $-7.1(9)$ \\
\hline C32 & $40.1(11)$ & $32.8(10)$ & 23.1(9) & $0.7(7)$ & $-10.8(8)$ & $-9.7(8)$ \\
\hline F3 & $56.7(9)$ & $37.0(7)$ & $42.9(7)$ & $-20.4(5)$ & $8.1(6)$ & $-12.2(6)$ \\
\hline 05 & $59.5(11)$ & $40.1(8)$ & $45.0(9)$ & $-17.2(7)$ & $-21.6(8)$ & $1.6(8)$ \\
\hline 06 & $50.1(10)$ & $26.5(7)$ & $47.2(9)$ & $-3.1(6)$ & $-2.8(7)$ & $-5.7(7)$ \\
\hline N3 & $34.3(9)$ & $26.2(8)$ & $21.5(7)$ & $1.3(6)$ & $1.2(6)$ & $1.0(7)$ \\
\hline C33 & $27.6(9)$ & $24.2(8)$ & $21.6(8)$ & $-2.2(6)$ & $-5.0(7)$ & $1.6(7)$ \\
\hline C34 & $29.3(10)$ & $27.8(9)$ & $41.3(11)$ & $-8.4(8)$ & $-5.4(8)$ & $0.8(8)$ \\
\hline C35 & $41.8(12)$ & $30.7(10)$ & $42.5(11)$ & $6.3(8)$ & $-4.1(9)$ & $-0.8(9)$ \\
\hline C36 & $37.1(11)$ & $33.2(10)$ & $29.5(9)$ & $6.8(8)$ & $-1.0(8)$ & $-2.8(9)$ \\
\hline C37 & $27.0(9)$ & $22.1(8)$ & $22.7(8)$ & $0.7(6)$ & $-4.1(7)$ & $1.1(7)$ \\
\hline C38 & $33.2(11)$ & $32.8(10)$ & $25.0(8)$ & $1.8(7)$ & $-7.9(8)$ & $-2.8(8)$ \\
\hline C39 & $33.2(11)$ & $41.8(12)$ & $45.2(12)$ & $4.0(9)$ & $-14.3(9)$ & $-5.8(9)$ \\
\hline $\mathrm{C} 40$ & $28.7(11)$ & $35.3(11)$ & $53.9(13)$ & $8.4(9)$ & $-0.2(9)$ & $-1.2(9)$ \\
\hline C41 & $37.8(12)$ & $33.4(10)$ & $32.5(10)$ & $3.1(8)$ & $6.2(8)$ & $2.0(9)$ \\
\hline $\mathrm{C} 42$ & $34.1(11)$ & $30.0(9)$ & $23.5(8)$ & $-1.3(7)$ & $-1.7(7)$ & $1.3(8)$ \\
\hline C43 & $23.2(9)$ & $31.2(9)$ & $20.7(8)$ & $-2.3(7)$ & $-2.1(6)$ & $-1.7(7)$ \\
\hline C44 & $38.3(11)$ & $39.6(11)$ & $21.0(8)$ & $0.4(7)$ & $-1.1(8)$ & $-8.1(9)$ \\
\hline $\mathrm{C} 45$ & $42.4(12)$ & $47.5(12)$ & $21.7(8)$ & $-9.8(8)$ & $3.3(8)$ & $-13.6(10)$ \\
\hline C46 & $33.4(11)$ & $31.2(10)$ & $32.5(10)$ & $-14.3(8)$ & $2.5(8)$ & $-7.4(8)$ \\
\hline C47 & $45.1(12)$ & $29.6(10)$ & $29.5(9)$ & $-2.8(7)$ & $-2.5(8)$ & $-1.8(9)$ \\
\hline C48 & $41.5(11)$ & $29.1(9)$ & $21.7(8)$ & $-3.4(7)$ & $-2.7(8)$ & $0.5(8)$ \\
\hline $\mathrm{F} 4$ & $77.3(11)$ & $56.0(8)$ & $18.6(6)$ & $-5.5(5)$ & $-12.4(6)$ & $0.0(7)$ \\
\hline 07 & $48.1(10)$ & $60.5(11)$ & $35.1(8)$ & $-24.8(8)$ & $8.6(7)$ & $-11.6(9)$ \\
\hline 08 & $63.3(12)$ & $54.8(10)$ & $19.8(6)$ & $2.1(6)$ & $-2.9(7)$ & $-21.9(9)$ \\
\hline $\mathrm{N} 4$ & $36.2(9)$ & $24.8(8)$ & $21.7(7)$ & $-2.2(6)$ & $-5.8(6)$ & $-10.0(7)$ \\
\hline C49 & $25.5(9)$ & $25.3(8)$ & $19.9(7)$ & $-4.3(6)$ & $-5.0(6)$ & $-2.3(7)$ \\
\hline C50 & $31.7(10)$ & $46.2(12)$ & $22.8(9)$ & $-8.0(8)$ & $-3.2(8)$ & $-11.7(9)$ \\
\hline C51 & $60.6(16)$ & $41.3(12)$ & $32.2(10)$ & $8.7(9)$ & $-15.6(10)$ & $-9.5(11)$ \\
\hline C52 & $40.3(11)$ & $26.6(9)$ & $28.1(9)$ & $1.2(7)$ & $-6.2(8)$ & $-8.4(8)$ \\
\hline C53 & $26.1(9)$ & $23.8(8)$ & $20.6(7)$ & $1.4(6)$ & $-6.3(7)$ & $-2.9(7)$ \\
\hline C54 & $28.5(10)$ & $30.8(9)$ & $34.0(9)$ & $3.4(7)$ & $-3.9(8)$ & $0.9(8)$ \\
\hline C55 & $25.7(11)$ & $52.0(14)$ & $51.2(13)$ & $18.6(10)$ & $-3.4(9)$ & $3.7(10)$ \\
\hline C56 & $30.4(12)$ & $65.4(16)$ & $53.2(13)$ & $27.8(12)$ & $-19.3(10)$ & $-17.5(11)$ \\
\hline C57 & $52.6(15)$ & $48.0(13)$ & $37.7(11)$ & $13.2(10)$ & $-24.6(10)$ & $-26.4(11)$ \\
\hline C58 & $39.0(11)$ & $28.6(9)$ & $25.7(8)$ & $1.7(7)$ & $-12.0(8)$ & $-8.1(8)$ \\
\hline C59 & $22.3(8)$ & $25.3(8)$ & $22.5(8)$ & $-4.3(6)$ & $-3.9(6)$ & $-2.1(7)$ \\
\hline C60 & $33.2(10)$ & $28.4(9)$ & $22.3(8)$ & $-3.1(7)$ & $-6.1(7)$ & $-7.2(8)$ \\
\hline C61 & $36.4(11)$ & $34.6(10)$ & $24.1(9)$ & $1.9(7)$ & $-4.6(8)$ & $-3.2(8)$ \\
\hline C62 & $40.9(12)$ & $42.5(11)$ & $17.9(8)$ & $-5.6(7)$ & $-7.8(8)$ & $2.9(9)$ \\
\hline C63 & $34.4(11)$ & $35.0(10)$ & $30.1(9)$ & $-12.3(8)$ & $-9.0(8)$ & $-0.4(8)$ \\
\hline C64 & $29.4(10)$ & $26.7(8)$ & $26.8(9)$ & $-5.9(7)$ & $-5.2(7)$ & $-4.4(7)$ \\
\hline
\end{tabular}


Table 3: Bond Lengths in Å for yp-6-29.

\begin{tabular}{|c|c|c|}
\hline Atom & Atom & Length/Å \\
\hline$\overline{F 1}$ & C14 & $1.363(2)$ \\
\hline 01 & $\mathrm{C} 2$ & $1.200(2)$ \\
\hline 02 & $\mathrm{C} 2$ & $1.328(3)$ \\
\hline 02 & $\mathrm{C} 3$ & $1.449(3)$ \\
\hline N1 & $\mathrm{C} 1$ & $1.455(2)$ \\
\hline N1 & $\mathrm{C} 4$ & $1.448(2)$ \\
\hline N1 & C11 & $1.408(2)$ \\
\hline $\mathrm{C} 1$ & $\mathrm{C} 2$ & $1.536(2)$ \\
\hline $\mathrm{C} 1$ & $\mathrm{C} 5$ & $1.539(2)$ \\
\hline $\mathrm{C} 3$ & $\mathrm{C} 4$ & $1.499(3)$ \\
\hline $\mathrm{C} 5$ & C6 & $1.395(2)$ \\
\hline $\mathrm{C} 5$ & $\mathrm{C} 10$ & $1.393(2)$ \\
\hline $\mathrm{C} 6$ & $\mathrm{C} 7$ & $1.392(3)$ \\
\hline $\mathrm{C} 7$ & $\mathrm{C} 8$ & $1.386(3)$ \\
\hline C8 & C9 & $1.387(3)$ \\
\hline $\mathrm{C} 9$ & $\mathrm{C} 10$ & $1.388(3)$ \\
\hline C11 & $\mathrm{C} 12$ & $1.397(3)$ \\
\hline C11 & C16 & $1.404(2)$ \\
\hline C12 & C13 & $1.388(3)$ \\
\hline C13 & C14 & $1.376(3)$ \\
\hline C14 & C15 & $1.373(3)$ \\
\hline C15 & C16 & $1.394(3)$ \\
\hline $\mathrm{F} 2$ & C30 & $1.371(2)$ \\
\hline 03 & C18 & $1.200(3)$ \\
\hline 04 & C18 & $1.335(3)$ \\
\hline 04 & C19 & $1.442(3)$ \\
\hline $\mathrm{N} 2$ & $\mathrm{C} 17$ & $1.450(2)$ \\
\hline N2 & $\mathrm{C} 20$ & $1.445(2)$ \\
\hline $\mathrm{N} 2$ & $\mathrm{C} 27$ & $1.394(2)$ \\
\hline C17 & C18 & $1.523(2)$ \\
\hline $\mathrm{C} 17$ & $\mathrm{C} 21$ & $1.534(2)$ \\
\hline C19 & $\mathrm{C} 20$ & $1.507(3)$ \\
\hline $\mathrm{C} 21$ & $\mathrm{C} 22$ & $1.390(3)$ \\
\hline $\mathrm{C} 21$ & $\mathrm{C} 26$ & $1.398(2)$ \\
\hline $\mathrm{C} 22$ & $\mathrm{C} 23$ & $1.386(3)$ \\
\hline $\mathrm{C} 23$ & $\mathrm{C} 24$ & $1.383(4)$ \\
\hline $\mathrm{C} 24$ & $\mathrm{C} 25$ & $1.381(4)$ \\
\hline $\mathrm{C} 25$ & $\mathrm{C} 26$ & $1.391(3)$ \\
\hline $\mathrm{C} 27$ & $\mathrm{C} 28$ & $1.406(2)$ \\
\hline $\mathrm{C} 27$ & C32 & $1.405(3)$ \\
\hline $\mathrm{C} 28$ & $\mathrm{C} 29$ & $1.389(3)$ \\
\hline $\mathrm{C} 29$ & $\mathrm{C} 30$ & $1.375(3)$ \\
\hline C30 & C31 & $1.374(3)$ \\
\hline C31 & $\mathrm{C} 32$ & $1.392(3)$ \\
\hline
\end{tabular}

\begin{tabular}{|c|c|c|}
\hline Atom & Atom & Length/Å \\
\hline$\overline{\mathrm{F} 3}$ & C46 & $1.368(2)$ \\
\hline 05 & C34 & $1.195(3)$ \\
\hline 06 & C34 & $1.335(3)$ \\
\hline 06 & C35 & $1.454(3)$ \\
\hline N3 & C33 & $1.447(2)$ \\
\hline N3 & C36 & $1.450(2)$ \\
\hline N3 & C43 & $1.394(2)$ \\
\hline C33 & C34 & $1.535(3)$ \\
\hline C33 & C37 & $1.536(3)$ \\
\hline C35 & C36 & $1.501(3)$ \\
\hline C37 & C38 & $1.392(3)$ \\
\hline C37 & $\mathrm{C} 42$ & $1.392(2)$ \\
\hline C38 & C39 & $1.391(3)$ \\
\hline C39 & $\mathrm{C} 40$ & $1.391(3)$ \\
\hline $\mathrm{C} 40$ & C41 & $1.385(3)$ \\
\hline C41 & C42 & $1.387(3)$ \\
\hline C43 & $\mathrm{C} 44$ & $1.405(2)$ \\
\hline C43 & C48 & $1.398(3)$ \\
\hline C44 & $\mathrm{C} 45$ & $1.391(3)$ \\
\hline C45 & $\mathrm{C} 46$ & $1.364(3)$ \\
\hline C46 & $\mathrm{C} 47$ & $1.374(3)$ \\
\hline C47 & C48 & $1.390(3)$ \\
\hline F4 & C62 & $1.372(2)$ \\
\hline 07 & $\mathrm{C} 50$ & $1.199(3)$ \\
\hline 08 & C50 & $1.334(3)$ \\
\hline 08 & C51 & $1.448(3)$ \\
\hline N4 & C49 & $1.452(2)$ \\
\hline N4 & C52 & $1.444(2)$ \\
\hline N4 & C59 & $1.387(2)$ \\
\hline C49 & $\mathrm{C} 50$ & $1.525(2)$ \\
\hline C49 & C53 & $1.533(2)$ \\
\hline C51 & C52 & $1.503(3)$ \\
\hline C53 & C54 & $1.390(3)$ \\
\hline C53 & C58 & $1.394(3)$ \\
\hline C54 & C55 & $1.391(3)$ \\
\hline C55 & C56 & $1.377(4)$ \\
\hline C56 & C57 & $1.381(4)$ \\
\hline C57 & C58 & $1.394(3)$ \\
\hline C59 & $\mathrm{C} 60$ & $1.405(3)$ \\
\hline C59 & C64 & $1.408(2)$ \\
\hline $\mathrm{C} 60$ & C61 & $1.389(2)$ \\
\hline C61 & C62 & $1.377(3)$ \\
\hline C62 & C63 & $1.370(3)$ \\
\hline C63 & C64 & $1.394(3)$ \\
\hline
\end{tabular}

Table 4: Bond Angles in ${ }^{\circ}$ for yp-6-29.

\begin{tabular}{lllc}
\hline Atom & Atom & Atom & Angle $^{\circ}$ \\
\hline C2 & O2 & C3 & $121.77(15)$ \\
C4 & N1 & C1 & $114.01(15)$ \\
C11 & N1 & C1 & $118.70(14)$ \\
C11 & N1 & C4 & $121.12(15)$ \\
N1 & C1 & C2 & $111.51(14)$ \\
N1 & C1 & C5 & $116.18(14)$
\end{tabular}

\begin{tabular}{lllc}
\hline Atom & Atom & Atom & Angle $^{\circ}$ \\
\hline C2 & C1 & C5 & $109.08(15)$ \\
O1 & C2 & O2 & $118.97(18)$ \\
O1 & C2 & C1 & $120.81(18)$ \\
O2 & C2 & C1 & $120.13(16)$ \\
O2 & C3 & C4 & $113.19(17)$ \\
N1 & C4 & C3 & $108.49(18)$
\end{tabular}




\begin{tabular}{|c|c|c|c|}
\hline Atom & Atom & Atom & Angle $/^{\circ}$ \\
\hline C6 & $\mathrm{C} 5$ & C1 & $122.05(15)$ \\
\hline C10 & $\mathrm{C} 5$ & $\mathrm{C} 1$ & $118.99(15)$ \\
\hline C10 & $\mathrm{C} 5$ & $\mathrm{C} 6$ & $118.94(17)$ \\
\hline $\mathrm{C} 7$ & $\mathrm{C} 6$ & $\mathrm{C} 5$ & $120.27(17)$ \\
\hline $\mathrm{C} 8$ & $\mathrm{C} 7$ & $\mathrm{C} 6$ & $120.00(18)$ \\
\hline $\mathrm{C} 7$ & $\mathrm{C} 8$ & $\mathrm{C} 9$ & $120.29(19)$ \\
\hline $\mathrm{C} 8$ & $\mathrm{C} 9$ & $\mathrm{C} 10$ & $119.54(17)$ \\
\hline $\mathrm{C} 9$ & $\mathrm{C} 10$ & $\mathrm{C} 5$ & $120.93(17)$ \\
\hline C12 & C11 & N1 & $120.01(15)$ \\
\hline C12 & C11 & C16 & $118.44(17)$ \\
\hline C16 & C11 & $\mathrm{N} 1$ & $121.46(17)$ \\
\hline C13 & $\mathrm{C} 12$ & $\mathrm{C} 11$ & $121.09(18)$ \\
\hline C14 & $\mathrm{C} 13$ & $\mathrm{C} 12$ & $118.69(19)$ \\
\hline $\mathrm{F} 1$ & C14 & C13 & $118.3(2)$ \\
\hline $\mathrm{F} 1$ & $\mathrm{C} 14$ & C15 & $119.33(19)$ \\
\hline C15 & $\mathrm{C} 14$ & C13 & $122.34(18)$ \\
\hline C14 & C15 & C16 & $118.90(18)$ \\
\hline C15 & C16 & $\mathrm{C} 11$ & $120.53(18)$ \\
\hline C18 & 04 & C19 & $117.03(16)$ \\
\hline C20 & $\mathrm{N} 2$ & $\mathrm{C} 17$ & $119.31(15)$ \\
\hline $\mathrm{C} 27$ & $\mathrm{~N} 2$ & $\mathrm{C} 17$ & $119.94(14)$ \\
\hline $\mathrm{C} 27$ & $\mathrm{~N} 2$ & $\mathrm{C} 20$ & $120.35(15)$ \\
\hline N2 & $\mathrm{C} 17$ & C18 & $112.53(15)$ \\
\hline $\mathrm{N} 2$ & C17 & $\mathrm{C} 21$ & $114.66(15)$ \\
\hline C18 & C17 & $\mathrm{C} 21$ & $109.28(14)$ \\
\hline 03 & C18 & 04 & $120.13(19)$ \\
\hline 03 & C18 & $\mathrm{C} 17$ & $121.8(2)$ \\
\hline 04 & C18 & $\mathrm{C} 17$ & $118.10(18)$ \\
\hline 04 & C19 & $\mathrm{C} 20$ & $110.8(2)$ \\
\hline $\mathrm{N} 2$ & $\mathrm{C} 20$ & C19 & $109.59(16)$ \\
\hline $\mathrm{C} 22$ & $\mathrm{C} 21$ & $\mathrm{C} 17$ & $120.89(16)$ \\
\hline $\mathrm{C} 22$ & $\mathrm{C} 21$ & $\mathrm{C} 26$ & $119.68(18)$ \\
\hline C26 & $\mathrm{C} 21$ & $\mathrm{C} 17$ & $119.29(17)$ \\
\hline C23 & $\mathrm{C} 22$ & $\mathrm{C} 21$ & $120.00(19)$ \\
\hline C24 & $\mathrm{C} 23$ & $\mathrm{C} 22$ & $120.2(2)$ \\
\hline $\mathrm{C} 25$ & $\mathrm{C} 24$ & $\mathrm{C} 23$ & $120.3(2)$ \\
\hline $\mathrm{C} 24$ & $\mathrm{C} 25$ & $\mathrm{C} 26$ & $120.0(2)$ \\
\hline $\mathrm{C} 25$ & $\mathrm{C} 26$ & $\mathrm{C} 21$ & $119.8(2)$ \\
\hline N2 & $\mathrm{C} 27$ & $\mathrm{C} 28$ & $120.86(16)$ \\
\hline $\mathrm{N} 2$ & $\mathrm{C} 27$ & $\mathrm{C} 32$ & $120.84(16)$ \\
\hline C32 & $\mathrm{C} 27$ & $\mathrm{C} 28$ & $118.29(16)$ \\
\hline C29 & $\mathrm{C} 28$ & $\mathrm{C} 27$ & $120.93(17)$ \\
\hline C30 & $\mathrm{C} 29$ & $\mathrm{C} 28$ & $118.65(17)$ \\
\hline F2 & $\mathrm{C} 30$ & $\mathrm{C} 29$ & $119.15(18)$ \\
\hline $\mathrm{F} 2$ & $\mathrm{C} 30$ & C31 & 118.24(19) \\
\hline C31 & $\mathrm{C} 30$ & $\mathrm{C} 29$ & $122.60(18)$ \\
\hline C30 & C31 & $\mathrm{C} 32$ & $118.80(19)$ \\
\hline C31 & C32 & $\mathrm{C} 27$ & $120.72(18)$ \\
\hline C34 & 06 & $\mathrm{C} 35$ & $115.43(16)$ \\
\hline C33 & N3 & $\mathrm{C} 36$ & $118.97(16)$ \\
\hline C43 & N3 & $\mathrm{C} 33$ & $120.13(14)$ \\
\hline C43 & N3 & $\mathrm{C} 36$ & $120.08(15)$ \\
\hline N3 & C33 & $\mathrm{C} 34$ & $111.36(15)$ \\
\hline N3 & C33 & $\mathrm{C} 37$ & $115.22(15)$ \\
\hline
\end{tabular}

\begin{tabular}{|c|c|c|c|}
\hline Atom & Atom & Atom & Angle $/^{\circ}$ \\
\hline$\overline{\mathrm{C} 34}$ & C33 & C37 & $109.85(15)$ \\
\hline 05 & C34 & 06 & $120.25(19)$ \\
\hline 05 & C34 & C33 & $121.7(2)$ \\
\hline 06 & C34 & C33 & $118.03(18)$ \\
\hline 06 & C35 & $\mathrm{C} 36$ & $111.01(19)$ \\
\hline N3 & C36 & $\mathrm{C} 35$ & $110.67(16)$ \\
\hline C38 & C37 & C33 & $121.75(16)$ \\
\hline $\mathrm{C} 42$ & C37 & C33 & $118.92(17)$ \\
\hline $\mathrm{C} 42$ & C37 & $\mathrm{C} 38$ & $119.32(18)$ \\
\hline C39 & C38 & $\mathrm{C} 37$ & $120.35(18)$ \\
\hline C38 & C39 & $\mathrm{C} 40$ & $119.8(2)$ \\
\hline C41 & $\mathrm{C} 40$ & C39 & $120.1(2)$ \\
\hline $\mathrm{C} 40$ & C41 & $\mathrm{C} 42$ & $120.00(19)$ \\
\hline C41 & $\mathrm{C} 42$ & $\mathrm{C} 37$ & $120.46(19)$ \\
\hline N3 & $\mathrm{C} 43$ & $\mathrm{C} 44$ & $120.33(17)$ \\
\hline N3 & $\mathrm{C} 43$ & $\mathrm{C} 48$ & $121.94(16)$ \\
\hline C48 & $\mathrm{C} 43$ & $\mathrm{C} 44$ & $117.73(18)$ \\
\hline $\mathrm{C} 45$ & C44 & $\mathrm{C} 43$ & $120.68(19)$ \\
\hline C46 & $\mathrm{C} 45$ & $\mathrm{C} 44$ & $119.41(18)$ \\
\hline F3 & C46 & $\mathrm{C} 47$ & $118.57(19)$ \\
\hline $\mathrm{C} 45$ & C46 & F3 & $119.38(18)$ \\
\hline $\mathrm{C} 45$ & C46 & $\mathrm{C} 47$ & $122.05(18)$ \\
\hline C46 & $\mathrm{C} 47$ & $\mathrm{C} 48$ & $118.69(19)$ \\
\hline $\mathrm{C} 47$ & $\mathrm{C} 48$ & $\mathrm{C} 43$ & $121.40(17)$ \\
\hline C50 & 08 & C51 & $116.69(15)$ \\
\hline C52 & N4 & $\mathrm{C} 49$ & $118.98(15)$ \\
\hline C59 & N4 & $\mathrm{C} 49$ & $120.30(14)$ \\
\hline C59 & N4 & $\mathrm{C} 52$ & $120.66(15)$ \\
\hline $\mathrm{N} 4$ & C49 & $\mathrm{C} 50$ & $112.22(15)$ \\
\hline N4 & C49 & C53 & $114.55(15)$ \\
\hline C50 & C49 & C53 & $109.24(14)$ \\
\hline 07 & C50 & 08 & $120.25(19)$ \\
\hline 07 & C50 & $\mathrm{C} 49$ & $121.4(2)$ \\
\hline 08 & C50 & $\mathrm{C} 49$ & $118.35(18)$ \\
\hline 08 & C51 & C52 & $110.6(2)$ \\
\hline N4 & $\mathrm{C} 52$ & C51 & $109.87(16)$ \\
\hline C54 & C53 & $\mathrm{C} 49$ & $121.14(16)$ \\
\hline C54 & C53 & $\mathrm{C} 58$ & $119.75(18)$ \\
\hline C58 & C53 & $\mathrm{C} 49$ & $119.05(17)$ \\
\hline C53 & C54 & C55 & $119.8(2)$ \\
\hline C56 & $\mathrm{C} 55$ & C54 & $120.5(2)$ \\
\hline C55 & C56 & C57 & $120.1(2)$ \\
\hline C56 & C57 & C58 & $120.2(2)$ \\
\hline C57 & C58 & C53 & $119.7(2)$ \\
\hline $\mathrm{N} 4$ & C59 & $\mathrm{C} 60$ & $121.28(15)$ \\
\hline $\mathrm{N} 4$ & C59 & C64 & $120.68(16)$ \\
\hline C60 & C59 & $\mathrm{C} 64$ & $118.04(16)$ \\
\hline C61 & C60 & C59 & $121.04(17)$ \\
\hline C62 & C61 & $\mathrm{C} 60$ & $118.70(18)$ \\
\hline F4 & C62 & C61 & $118.73(19)$ \\
\hline C63 & C62 & $\mathrm{F} 4$ & $118.68(18)$ \\
\hline C63 & C62 & C61 & $122.59(17)$ \\
\hline C62 & C63 & $\mathrm{C} 64$ & 118.78(17) \\
\hline C63 & C64 & C59 & $120.82(17)$ \\
\hline
\end{tabular}


Table 5: Torsion Angles in ${ }^{\circ}$ for yp-6-29.

\begin{tabular}{|c|c|c|c|c|}
\hline Atom & Atom & Atom & Atom & Angle $/^{\circ}$ \\
\hline$\overline{\mathrm{F} 1}$ & C14 & C15 & $\mathrm{C} 16$ & $\begin{array}{c}-179.63(18 \\
)\end{array}$ \\
\hline 02 & $\mathrm{C} 3$ & $\mathrm{C} 4$ & $\mathrm{~N} 1$ & $51.0(3)$ \\
\hline N1 & $\mathrm{C} 1$ & $\mathrm{C} 2$ & 01 & $161.47(19)$ \\
\hline N1 & $\mathrm{C} 1$ & $\mathrm{C} 2$ & 02 & $-21.9(3)$ \\
\hline N1 & $\mathrm{C} 1$ & $\mathrm{C} 5$ & $\mathrm{C} 6$ & $4.2(2)$ \\
\hline N1 & $\mathrm{C} 1$ & $\mathrm{C} 5$ & C10 & $\begin{array}{c}-174.10(15 \\
)\end{array}$ \\
\hline N1 & C11 & $\mathrm{C} 12$ & $\mathrm{C} 13$ & $176.22(18)$ \\
\hline N1 & C11 & C16 & C15 & $-176.05(17$ \\
\hline $\mathrm{C} 1$ & $\mathrm{~N} 1$ & $\mathrm{C} 4$ & $\mathrm{C} 3$ & $-61.4(2)$ \\
\hline $\mathrm{C} 1$ & N1 & C11 & C12 & $44.0(3)$ \\
\hline $\mathrm{C} 1$ & N1 & C11 & C16 & $\begin{array}{c}-139.40(18 \\
)\end{array}$ \\
\hline $\mathrm{C} 1$ & $\mathrm{C} 5$ & $\mathrm{C} 6$ & $\mathrm{C} 7$ & $\begin{array}{c}-179.92(17 \\
)\end{array}$ \\
\hline $\mathrm{C} 1$ & $\mathrm{C} 5$ & $\mathrm{C} 10$ & $\mathrm{C} 9$ & $179.40(16)$ \\
\hline $\mathrm{C} 2$ & 02 & $\mathrm{C} 3$ & $\mathrm{C} 4$ & $-29.8(4)$ \\
\hline $\mathrm{C} 2$ & $\mathrm{C} 1$ & $\mathrm{C} 5$ & $\mathrm{C} 6$ & $\begin{array}{c}-122.82(18 \\
)\end{array}$ \\
\hline $\mathrm{C} 2$ & $\mathrm{C} 1$ & $\mathrm{C} 5$ & $\mathrm{C} 10$ & $58.9(2)$ \\
\hline $\mathrm{C} 3$ & 02 & $\mathrm{C} 2$ & 01 & $-168.3(2)$ \\
\hline $\mathrm{C} 3$ & 02 & $\mathrm{C} 2$ & $\mathrm{C} 1$ & $15.0(3)$ \\
\hline $\mathrm{C} 4$ & N1 & $\mathrm{C} 1$ & $\mathrm{C} 2$ & $46.1(2)$ \\
\hline $\mathrm{C} 4$ & $\mathrm{~N} 1$ & $\mathrm{C} 1$ & $\mathrm{C} 5$ & $-79.7(2)$ \\
\hline $\mathrm{C} 4$ & N1 & C11 & C12 & $\begin{array}{c}-165.31(18 \\
)\end{array}$ \\
\hline $\mathrm{C} 4$ & $\mathrm{~N} 1$ & C11 & C16 & $11.3(3)$ \\
\hline $\mathrm{C} 5$ & $\mathrm{C} 1$ & $\mathrm{C} 2$ & 01 & $-68.9(2)$ \\
\hline $\mathrm{C} 5$ & $\mathrm{C} 1$ & $\mathrm{C} 2$ & 02 & $107.8(2)$ \\
\hline $\mathrm{C} 5$ & $\mathrm{C} 6$ & $\mathrm{C} 7$ & $\mathrm{C} 8$ & $1.2(3)$ \\
\hline $\mathrm{C} 6$ & $\mathrm{C} 5$ & $\mathrm{C} 10$ & $\mathrm{C} 9$ & $1.0(3)$ \\
\hline $\mathrm{C} 6$ & $\mathrm{C} 7$ & $\mathrm{C} 8$ & $\mathrm{C} 9$ & $-0.1(3)$ \\
\hline $\mathrm{C} 7$ & $\mathrm{C} 8$ & $\mathrm{C} 9$ & $\mathrm{C} 10$ & $-0.4(3)$ \\
\hline C8 & $\mathrm{C9}$ & C10 & $\mathrm{C} 5$ & $0.0(3)$ \\
\hline C10 & $\mathrm{C} 5$ & $\mathrm{C} 6$ & $\mathrm{C} 7$ & $-1.6(3)$ \\
\hline $\mathrm{C} 11$ & N1 & $\mathrm{C} 1$ & $\mathrm{C} 2$ & $\begin{array}{c}-161.16(16 \\
)\end{array}$ \\
\hline C11 & N1 & $\mathrm{C} 1$ & $\mathrm{C} 5$ & $73.0(2)$ \\
\hline C11 & $\mathrm{N} 1$ & $\mathrm{C} 4$ & $\mathrm{C} 3$ & $146.59(19)$ \\
\hline C11 & $\mathrm{C} 12$ & C13 & C14 & $0.2(3)$ \\
\hline C12 & C11 & C16 & C15 & $0.6(3)$ \\
\hline C12 & $\mathrm{C} 13$ & C14 & $\mathrm{F} 1$ & $179.75(19)$ \\
\hline C12 & $\mathrm{C} 13$ & C14 & C15 & $0.1(3)$ \\
\hline C13 & $\mathrm{C} 14$ & C15 & C16 & $0.1(3)$ \\
\hline C14 & C15 & C16 & $\mathrm{C} 11$ & $-0.4(3)$ \\
\hline C16 & $\mathrm{C} 11$ & $\mathrm{C} 12$ & C13 & $-0.5(3)$ \\
\hline $\mathrm{F} 2$ & C30 & C31 & C32 & $178.9(2)$ \\
\hline 04 & C19 & $\mathrm{C} 20$ & N2 & $-57.5(3)$ \\
\hline $\mathrm{N} 2$ & $\mathrm{C} 17$ & C18 & 03 & $145.4(2)$ \\
\hline $\mathrm{N} 2$ & $\mathrm{C} 17$ & C18 & 04 & $-36.1(2)$ \\
\hline $\mathrm{N} 2$ & $\mathrm{C} 17$ & $\mathrm{C} 21$ & $\mathrm{C} 22$ & $1.0(2)$ \\
\hline N2 & $\mathrm{C} 17$ & $\mathrm{C} 21$ & $\mathrm{C} 26$ & $\begin{array}{c}-174.78(15 \\
)\end{array}$ \\
\hline $\mathrm{N} 2$ & $\mathrm{C} 27$ & $\mathrm{C} 28$ & C29 & $179.02(19)$ \\
\hline
\end{tabular}




\begin{tabular}{|c|c|c|c|c|}
\hline Atom & Atom & Atom & Atom & Angle ${ }^{\circ}$ \\
\hline $\mathrm{N} 2$ & C27 & C32 & C31 & $-178.2(2)$ \\
\hline C17 & $\mathrm{N} 2$ & $\mathrm{C} 20$ & C19 & $20.8(3)$ \\
\hline C17 & N2 & $\mathrm{C} 27$ & C28 & 166.71(18) \\
\hline C17 & N2 & $\mathrm{C} 27$ & C32 & $-14.3(3)$ \\
\hline C17 & $\mathrm{C} 21$ & $\mathrm{C} 22$ & $\mathrm{C} 23$ & $\begin{array}{c}-174.29(17 \\
)\end{array}$ \\
\hline C17 & $\mathrm{C} 21$ & $\mathrm{C} 26$ & $\mathrm{C} 25$ & $174.06(17)$ \\
\hline C18 & 04 & C19 & C20 & $48.0(3)$ \\
\hline C18 & C17 & $\mathrm{C} 21$ & C22 & $\begin{array}{c}-126.43(18 \\
)\end{array}$ \\
\hline C18 & C17 & $\mathrm{C} 21$ & $\mathrm{C} 26$ & $57.8(2)$ \\
\hline C19 & 04 & C18 & 03 & $178.0(2)$ \\
\hline C19 & 04 & C18 & C17 & $-0.4(3)$ \\
\hline C20 & N2 & C17 & C18 & $23.5(3)$ \\
\hline C20 & N2 & C17 & $\mathrm{C} 21$ & $-102.3(2)$ \\
\hline $\mathrm{C} 20$ & N2 & $\mathrm{C} 27$ & $\mathrm{C} 28$ & $-6.0(3)$ \\
\hline $\mathrm{C} 20$ & N2 & $\mathrm{C} 27$ & C32 & $173.0(2)$ \\
\hline $\mathrm{C} 21$ & C17 & C18 & 03 & $-86.0(2)$ \\
\hline C21 & C17 & C18 & 04 & $92.5(2)$ \\
\hline $\mathrm{C} 21$ & $\mathrm{C} 22$ & $\mathrm{C} 23$ & C24 & $0.3(3)$ \\
\hline C22 & $\mathrm{C} 21$ & $\mathrm{C} 26$ & $\mathrm{C} 25$ & $-1.7(3)$ \\
\hline $\mathrm{C} 22$ & $\mathrm{C} 23$ & $\mathrm{C} 24$ & $\mathrm{C} 25$ & $-1.8(3)$ \\
\hline C23 & C24 & $\mathrm{C} 25$ & C26 & $1.5(3)$ \\
\hline C24 & C25 & $\mathrm{C} 26$ & $\mathrm{C} 21$ & $0.3(3)$ \\
\hline $\mathrm{C} 26$ & $\mathrm{C} 21$ & $\mathrm{C} 22$ & $\mathrm{C} 23$ & $1.4(3)$ \\
\hline $\mathrm{C} 27$ & N2 & C17 & C18 & $\begin{array}{c}-149.33(18 \\
)\end{array}$ \\
\hline C27 & N2 & C17 & $\mathrm{C} 21$ & $85.0(2)$ \\
\hline $\mathrm{C} 27$ & $\mathrm{~N} 2$ & $\mathrm{C} 20$ & C19 & $-166.4(2)$ \\
\hline C27 & $\mathrm{C} 28$ & $\mathrm{C} 29$ & C30 & $-0.8(3)$ \\
\hline C28 & $\mathrm{C} 27$ & C32 & C31 & $0.8(3)$ \\
\hline C28 & C29 & C30 & F2 & $\begin{array}{c}-178.12(19 \\
)\end{array}$ \\
\hline C28 & C29 & C30 & C31 & $0.9(3)$ \\
\hline C29 & C30 & C31 & C32 & $-0.1(4)$ \\
\hline C30 & C31 & C32 & C27 & $-0.7(4)$ \\
\hline C32 & $\mathrm{C} 27$ & $\mathrm{C} 28$ & C29 & $0.0(3)$ \\
\hline F3 & C46 & C47 & C48 & $179.3(2)$ \\
\hline 06 & C35 & C36 & N3 & $-52.2(2)$ \\
\hline N3 & C33 & C34 & 05 & $145.0(2)$ \\
\hline N3 & C33 & C34 & 06 & $-35.4(3)$ \\
\hline N3 & C33 & C37 & C38 & $8.5(2)$ \\
\hline N3 & C33 & C37 & C42 & $\begin{array}{c}-170.58(16 \\
)\end{array}$ \\
\hline N3 & C43 & C44 & C45 & $-179.8(2)$ \\
\hline N3 & C43 & C48 & C47 & $178.3(2)$ \\
\hline C33 & N3 & C36 & C35 & $6.9(3)$ \\
\hline C33 & N3 & C43 & C44 & $\begin{array}{c}-170.50(19 \\
)\end{array}$ \\
\hline C33 & N3 & C43 & C48 & $9.4(3)$ \\
\hline C33 & C37 & C38 & C39 & $\begin{array}{c}-179.59(18 \\
)\end{array}$ \\
\hline C33 & C37 & C42 & C41 & $179.02(17)$ \\
\hline C34 & 06 & C35 & C36 & $54.3(2)$ \\
\hline C34 & C33 & C37 & C38 & $\begin{array}{c}-118.25(19 \\
)\end{array}$ \\
\hline C34 & C33 & C37 & C42 & $62.7(2)$ \\
\hline C35 & 06 & C34 & 05 & $170.5(2)$ \\
\hline
\end{tabular}




\begin{tabular}{|c|c|c|c|c|}
\hline Atom & Atom & Atom & Atom & Angle $/^{\circ}$ \\
\hline$\overline{C 35}$ & 06 & C34 & C33 & $-9.1(3)$ \\
\hline C36 & N3 & C33 & C34 & $35.1(2)$ \\
\hline C36 & N3 & C33 & C37 & $-90.8(2)$ \\
\hline C36 & N3 & $\mathrm{C} 43$ & $\mathrm{C} 44$ & $-1.0(3)$ \\
\hline C36 & N3 & $\mathrm{C} 43$ & $\mathrm{C} 48$ & $178.9(2)$ \\
\hline C37 & C33 & C34 & 05 & $-86.1(2)$ \\
\hline C37 & C33 & C34 & 06 & $93.5(2)$ \\
\hline C37 & C38 & C39 & $\mathrm{C} 40$ & $0.6(3)$ \\
\hline C38 & $\mathrm{C} 37$ & $\mathrm{C} 42$ & $\mathrm{C} 41$ & $-0.1(3)$ \\
\hline C38 & C39 & $\mathrm{C} 40$ & $\mathrm{C} 41$ & $0.0(3)$ \\
\hline C39 & $\mathrm{C} 40$ & $\mathrm{C} 41$ & $\mathrm{C} 42$ & $-0.6(3)$ \\
\hline $\mathrm{C} 40$ & $\mathrm{C} 41$ & $\mathrm{C} 42$ & $\mathrm{C} 37$ & $0.6(3)$ \\
\hline C42 & $\mathrm{C} 37$ & C38 & C39 & $-0.5(3)$ \\
\hline C43 & N3 & C33 & C34 & $\begin{array}{c}-155.24(17 \\
)\end{array}$ \\
\hline C43 & N3 & C33 & $\mathrm{C} 37$ & $78.8(2)$ \\
\hline C43 & N3 & C36 & C35 & $\begin{array}{c}-162.75(18 \\
)\end{array}$ \\
\hline C43 & $\mathrm{C} 44$ & $\mathrm{C} 45$ & $\mathrm{C} 46$ & $1.1(3)$ \\
\hline C44 & $\mathrm{C} 43$ & $\mathrm{C} 48$ & $\mathrm{C} 47$ & $-1.9(3)$ \\
\hline C44 & $\mathrm{C} 45$ & $\mathrm{C} 46$ & F3 & $179.2(2)$ \\
\hline C44 & $\mathrm{C} 45$ & C46 & $\mathrm{C} 47$ & $-1.2(3)$ \\
\hline $\mathrm{C} 45$ & $\mathrm{C} 46$ & $\mathrm{C} 47$ & $\mathrm{C} 48$ & $-0.3(4)$ \\
\hline C46 & $\mathrm{C} 47$ & $\mathrm{C} 48$ & $\mathrm{C} 43$ & $1.9(3)$ \\
\hline C48 & $\mathrm{C} 43$ & C44 & $\mathrm{C} 45$ & $0.3(3)$ \\
\hline F4 & C62 & C63 & C64 & $\begin{array}{c}-179.22(19 \\
)\end{array}$ \\
\hline 08 & C51 & C52 & N4 & $-58.2(2)$ \\
\hline $\mathrm{N} 4$ & $\mathrm{C} 49$ & C50 & 07 & $143.9(2)$ \\
\hline $\mathrm{N} 4$ & $\mathrm{C} 49$ & C50 & 08 & $-37.3(3)$ \\
\hline N4 & C49 & C53 & C54 & $6.0(2)$ \\
\hline $\mathrm{N} 4$ & $\mathrm{C} 49$ & C53 & C58 & $\begin{array}{c}-171.09(15 \\
)\end{array}$ \\
\hline N4 & C59 & C60 & C61 & $\begin{array}{c}-178.19(19 \\
)\end{array}$ \\
\hline $\mathrm{N} 4$ & C59 & C64 & C63 & $178.18(19)$ \\
\hline C49 & N4 & C52 & C51 & $21.8(3)$ \\
\hline C49 & $\mathrm{N} 4$ & C59 & $\mathrm{C} 60$ & $-9.3(3)$ \\
\hline C49 & $\mathrm{N} 4$ & C59 & C64 & $171.35(18)$ \\
\hline C49 & $\mathrm{C} 53$ & C54 & C55 & $\begin{array}{c}-176.35(18 \\
)\end{array}$ \\
\hline C49 & C53 & C58 & C57 & $175.83(17)$ \\
\hline C50 & 08 & C51 & $\mathrm{C} 52$ & $47.1(3)$ \\
\hline C50 & $\mathrm{C} 49$ & C53 & $\mathrm{C} 54$ & $\begin{array}{c}-120.87(19 \\
)\end{array}$ \\
\hline $\mathrm{C} 50$ & $\mathrm{C} 49$ & C53 & C58 & $62.1(2)$ \\
\hline C51 & 08 & $\mathrm{C} 50$ & 07 & $180.0(2)$ \\
\hline C51 & 08 & C50 & C49 & $1.2(3)$ \\
\hline C52 & $\mathrm{N} 4$ & C49 & $\mathrm{C} 50$ & $23.3(3)$ \\
\hline $\mathrm{C} 52$ & $\mathrm{~N} 4$ & $\mathrm{C} 49$ & C53 & $-102.0(2)$ \\
\hline C52 & $\mathrm{N} 4$ & C59 & $\mathrm{C} 60$ & $173.70(19)$ \\
\hline C52 & $\mathrm{N} 4$ & C59 & C64 & $-5.6(3)$ \\
\hline C53 & $\mathrm{C} 49$ & C50 & 07 & $-87.9(2)$ \\
\hline C53 & C49 & C50 & 08 & $90.8(2)$ \\
\hline C53 & C54 & C55 & C56 & $0.4(3)$ \\
\hline C54 & C53 & C58 & C57 & $-1.3(3)$ \\
\hline C54 & C55 & C56 & $\mathrm{C} 57$ & $-0.9(3)$ \\
\hline C55 & C56 & C57 & C58 & $0.3(3)$ \\
\hline
\end{tabular}




\begin{tabular}{llllr}
\hline Atom & Atom & Atom & Atom & Angle $/^{\circ}$ \\
\hline C56 & C57 & C58 & C53 & $0.8(3)$ \\
C58 & C53 & C54 & C55 & $0.7(3)$ \\
C59 & N4 & C49 & C50 & $-153.72(18$ \\
& & & & ) \\
C59 & N4 & C49 & C53 & $81.0(2)$ \\
C59 & N4 & C52 & C51 & $-161.2(2)$ \\
C59 & C60 & C61 & C62 & $-0.1(3)$ \\
C60 & C59 & C64 & C63 & $-1.2(3)$ \\
C60 & C61 & C62 & F4 & $179.2(2)$ \\
C60 & C61 & C62 & C63 & $-1.1(3)$ \\
C61 & C62 & C63 & C64 & $1.1(3)$ \\
C62 & C63 & C64 & C59 & $0.1(3)$ \\
C64 & C59 & C60 & C61 & $1.2(3)$
\end{tabular}


Table 6: Hydrogen Fractional Atomic Coordinates $\left(\times 10^{4}\right)$ and Equivalent Isotropic Displacement Parameters $\left(\AA^{2} \times 10^{3}\right)$ for $\mathbf{y p - 6 - 2 9}$. $U_{e q}$ is defined as $1 / 3$ of the trace of the orthogonalised $U_{i j .}$.

\begin{tabular}{|c|c|c|c|c|}
\hline Atom & $\mathbf{x}$ & $\mathbf{y}$ & $\mathbf{z}$ & $U_{e q}$ \\
\hline$\overline{\mathrm{H} 1}$ & 7804.42 & 6117.1 & -793.6 & 27 \\
\hline $\mathrm{H} 3 \mathrm{~A}$ & 7130.39 & 2149.63 & 642.95 & 59 \\
\hline H3B & 5577.02 & 3223.14 & 495.61 & 59 \\
\hline $\mathrm{H} 4 \mathrm{~A}$ & 7080.51 & 4263.86 & 1302.21 & 40 \\
\hline $\mathrm{H} 4 \mathrm{~B}$ & 8918.07 & 3884.78 & 737.56 & 40 \\
\hline H6 & 10662.16 & 5566.75 & 454.51 & 32 \\
\hline H7 & 13676.43 & 5782.42 & 160.99 & 39 \\
\hline H8 & 15119.35 & 5885.92 & -1143.21 & 39 \\
\hline H9 & 13564.94 & 5757.7 & -2160.79 & 34 \\
\hline H10 & 10565.7 & 5505.41 & -1867.77 & 30 \\
\hline H12 & 6485.2 & 7773.69 & -244.17 & 35 \\
\hline H13 & 5901.33 & 9883.66 & 379.3 & 42 \\
\hline H15 & 7273.07 & 8155.24 & 2341.44 & 40 \\
\hline H16 & 7872.96 & 6034.03 & 1721.21 & 34 \\
\hline H17 & 2412.74 & 3810.61 & 3193.36 & 28 \\
\hline H19A & 1010.46 & 7077.33 & 2190.36 & 52 \\
\hline H19B & 2700.68 & 7877.39 & 1824.81 & 52 \\
\hline $\mathrm{H} 20 \mathrm{~A}$ & 4083.17 & 7079.52 & 2859.79 & 39 \\
\hline H20B & 2239.44 & 7858.7 & 3213.33 & 39 \\
\hline $\mathrm{H} 22$ & -988.14 & 6154.25 & 3566.26 & 34 \\
\hline $\mathrm{H} 23$ & -3921.85 & 5714.83 & 3689.79 & 47 \\
\hline $\mathrm{H} 24$ & -4687.58 & 3727.73 & 3177.34 & 51 \\
\hline $\mathrm{H} 25$ & -2542.25 & 2227.48 & 2480.97 & 46 \\
\hline $\mathrm{H} 26$ & 405.58 & 2661.81 & 2341.41 & 33 \\
\hline $\mathrm{H} 28$ & 3385.98 & 7486.15 & 4259.9 & 33 \\
\hline H29 & 3327.67 & 7303.27 & 5612.93 & 38 \\
\hline H31 & 994.8 & 3734.79 & 5706.33 & 44 \\
\hline H32 & 1099.97 & 3875.14 & 4347.13 & 37 \\
\hline H33 & 7722.76 & 1048.5 & 4272.59 & 29 \\
\hline H35A & 9734.57 & -1751.11 & 5400.34 & 47 \\
\hline Н35В & 8287.57 & -2690.64 & 5879.54 & 47 \\
\hline H36A & 6288.62 & -825.66 & 6135.29 & 41 \\
\hline H36B & 8060.21 & -429.65 & 6381.01 & 41 \\
\hline H38 & 10803.61 & 646.2 & 5439.73 & 36 \\
\hline H39 & 13793.17 & 913.29 & 5050.24 & 47 \\
\hline $\mathrm{H} 40$ & 15047.82 & 957.13 & 3714.06 & 49 \\
\hline $\mathrm{H} 41$ & 13319.81 & 733.47 & 2773.36 & 44 \\
\hline $\mathrm{H} 42$ & 10348.61 & 428.67 & 3163 & 36 \\
\hline $\mathrm{H} 44$ & 6692.83 & 1291.2 & 6848.26 & 40 \\
\hline $\mathrm{H} 45$ & 6079.67 & 3504.62 & 7340.63 & 45 \\
\hline $\mathrm{H} 47$ & 6895.66 & 5096.58 & 5140.61 & 42 \\
\hline $\mathrm{H} 48$ & 7633.99 & 2900.64 & 4638.59 & 37 \\
\hline $\mathrm{H} 49$ & 2036.44 & -1204.99 & 8193.3 & 28 \\
\hline H51A & 301.28 & 2088.67 & 7327.92 & 52 \\
\hline H51B & 1896.78 & 2992.4 & 6963.82 & 52 \\
\hline H52A & 3446.87 & 2147.67 & 7934.14 & 38 \\
\hline H52B & 1586.01 & 2834.91 & 8342.77 & 38 \\
\hline H54 & -1515.4 & 1063.03 & 8601.4 & 38 \\
\hline H55 & -4420.99 & 539.07 & 8706.64 & 53 \\
\hline H56 & -5081.49 & -1476.48 & 8195.35 & 58 \\
\hline H57 & -2853.64 & -2963.41 & 7544.43 & 52 \\
\hline H58 & 65.41 & -2442.38 & 7412.27 & 36 \\
\hline $\mathrm{H} 60$ & 792.84 & -1286.76 & 9356.12 & 33 \\
\hline
\end{tabular}




\begin{tabular}{lccccc}
\hline Atom & $\mathbf{x}$ & $\mathbf{y}$ & $\mathbf{z}$ & & $\boldsymbol{U}_{\text {eq }}$ \\
\hline H61 & 612.11 & -1489.17 & 10715.76 & 38 & \\
H63 & 2397.11 & 2275.48 & 10745.06 & 39 & \\
H64 & 2589.32 & 2498.01 & 9382.23 & 33 &
\end{tabular}




\section{Crystallographic data for $1 \mathrm{k}$}<smiles></smiles>

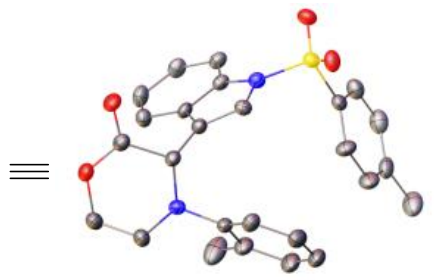

\begin{tabular}{|c|c|}
\hline Compound & YP-6-98 \\
\hline Formula & $\mathrm{C}_{26} \mathrm{H}_{24} \mathrm{~N}_{2} \mathrm{O}_{4} \mathrm{~S}$ \\
\hline$D_{\text {calc. }} / \mathrm{g} \mathrm{cm}^{-3}$ & 1.315 \\
\hline$\mu / \mathrm{mm}^{-1}$ & 1.528 \\
\hline Formula Weight & 460.53 \\
\hline Colour & clear colourless \\
\hline Shape & plate \\
\hline $\mathrm{Size} / \mathrm{mm}^{3}$ & $0.35 \times 0.16 \times 0.04$ \\
\hline$T / \mathrm{K}$ & $140.00(10)$ \\
\hline Crystal System & orthorhombic \\
\hline Flack Parameter & $0.016(8)$ \\
\hline Space Group & $P 2_{1} 2_{1} 2_{1}$ \\
\hline$a / \AA ̊$ & $8.58380(17)$ \\
\hline$b / \AA$ & $32.7737(5)$ \\
\hline$c / \AA$ & $33.0649(7)$ \\
\hline$\left.\alpha\right|^{\circ}$ & 90 \\
\hline$\beta /^{\circ}$ & 90 \\
\hline$\gamma /{ }^{\circ}$ & 90 \\
\hline $\mathrm{V} / \AA^{3}$ & $9301.9(3)$ \\
\hline$Z$ & 16 \\
\hline$Z^{\prime}$ & 4 \\
\hline Wavelength/Å & 1.54184 \\
\hline Radiation type & $\mathrm{Cu} K \alpha$ \\
\hline$\Theta_{\min } /^{\circ}$ & 3.010 \\
\hline$\Theta_{\max } /^{\circ}$ & 72.852 \\
\hline Measured Refl's. & 76968 \\
\hline Indep't Refl's & 18198 \\
\hline Refl's I $\geq 2 \sigma(\mathrm{I})$ & 15928 \\
\hline$R_{\text {int }}$ & 0.0570 \\
\hline Parameters & 1197 \\
\hline Restraints & 1144 \\
\hline Largest Peak/e $\AA^{-3}$ & 1.121 \\
\hline Deepest Hole/e $\AA^{-3}$ & -0.625 \\
\hline GooF & 1.073 \\
\hline$w R_{2}$ (all data) & 0.2843 \\
\hline$w R_{2}$ & 0.2760 \\
\hline$R_{1}$ (all data) & 0.0963 \\
\hline$R_{1}$ & 0.0881 \\
\hline
\end{tabular}


Table 7: Fractional Atomic Coordinates $\left(\times 10^{4}\right)$ and Equivalent Isotropic Displacement Parameters $\left(\AA^{2} \times 10^{3}\right)$ for yp-6-98. $U_{e q}$ is defined as $1 / 3$ of the trace of the orthogonalised $U_{i j}$.

\begin{tabular}{|c|c|c|c|c|}
\hline Atom & $\mathbf{x}$ & $\mathbf{y}$ & $\mathbf{z}$ & $U_{e q}$ \\
\hline$\overline{\mathrm{S} 1}$ & $7884(2)$ & $3531.2(5)$ & $7056.6(5)$ & $38.8(4)$ \\
\hline 01 & $6956(8)$ & $3233.7(18)$ & $7260.0(17)$ & $49.8(14)$ \\
\hline 02 & $9518(7)$ & $3480(2)$ & $7012.9(17)$ & $48.0(13)$ \\
\hline 03 & $6206(8)$ & $3795.9(19)$ & 4879.8(17) & 49.7(14) \\
\hline 04 & $6977(9)$ & $3247.3(18)$ & $5191.3(19)$ & $53.8(15)$ \\
\hline N1 & $7222(8)$ & $3559.4(19)$ & 6585.6(19) & $39.6(13)$ \\
\hline N2 & 7498(8) & $4306.2(19)$ & 5469.1(19) & 39.1(13) \\
\hline $\mathrm{C} 1$ & $8146(9)$ & $3661(2)$ & $6251(2)$ & $36.5(14)$ \\
\hline $\mathrm{C} 2$ & $7210(9)$ & $3736(2)$ & $5924(2)$ & $36.9(14)$ \\
\hline $\mathrm{C} 3$ & $5632(9)$ & $3682(2)$ & $6060(2)$ & $37.6(14)$ \\
\hline $\mathrm{C} 4$ & 4193(10) & $3715(3)$ & $5860(2)$ & $43.6(16)$ \\
\hline $\mathrm{C} 5$ & 2861(10) & $3631(3)$ & $6081(3)$ & $50.3(19)$ \\
\hline C6 & $2908(10)$ & $3525(3)$ & $6490(3)$ & $47.0(17)$ \\
\hline $\mathrm{C} 7$ & $4315(10)$ & $3492(2)$ & $6691(2)$ & $41.9(16)$ \\
\hline C8 & $5651(9)$ & $3576(2)$ & $6468(2)$ & $35.8(14)$ \\
\hline $\mathrm{C} 9$ & $7757(9)$ & $3865(2)$ & $5513(2)$ & $37.4(14)$ \\
\hline C10 & $6930(10)$ & $3616(2)$ & $5186(2)$ & $42.5(16)$ \\
\hline C11 & 6171(13) & $4234(3)$ & $4843(3)$ & $55(2)$ \\
\hline $\mathrm{C} 12$ & 7548(11) & $4430(3)$ & $5048(2)$ & $48.7(19)$ \\
\hline C13 & $8479(10)$ & $4546(2)$ & $5733(2)$ & $40.3(16)$ \\
\hline C14 & $7739(13)$ & $4847(3)$ & $5969(3)$ & $55(2)$ \\
\hline C15 & $8654(15)$ & $5076(3)$ & $6238(3)$ & $65(3)$ \\
\hline C16 & $10235(14)$ & $5003(3)$ & $6267(3)$ & $62(2)$ \\
\hline C17 & $10953(13)$ & $4713(3)$ & $6026(3)$ & $59(2)$ \\
\hline C18 & $10063(11)$ & $4488(3)$ & $5758(3)$ & $48.7(19)$ \\
\hline C19 & $6034(15)$ & $4924(4)$ & $5938(5)$ & $91(5)$ \\
\hline $\mathrm{C} 20$ & $7518(9)$ & $4012(2)$ & $7269(2)$ & $39.2(15)$ \\
\hline $\mathrm{C} 21$ & 8233(11) & $4359(2)$ & $7114(3)$ & $49.8(19)$ \\
\hline $\mathrm{C} 22$ & 7981(14) & $4728(3)$ & $7289(3)$ & $59(2)$ \\
\hline C23 & $7023(12)$ & $4763(3)$ & $7624(3)$ & $56(2)$ \\
\hline $\mathrm{C} 24$ & $6291(12)$ & $4413(4)$ & $7777(3)$ & $60(2)$ \\
\hline $\mathrm{C} 25$ & $6540(11)$ & $4038(3)$ & $7599(3)$ & $52(2)$ \\
\hline C26 & $6775(17)$ & $5177(4)$ & $7822(4)$ & $79(4)$ \\
\hline S2 & $9338(2)$ & $6100.4(6)$ & $6896.9(6)$ & $43.6(4)$ \\
\hline 05 & $8770(9)$ & $5762.3(18)$ & $7121(2)$ & $56.4(16)$ \\
\hline 06 & $10963(7)$ & $6134(2)$ & $6803(2)$ & 62.1(18) \\
\hline 07 & $6525(9)$ & $6341(2)$ & $4791.9(19)$ & $62.2(17)$ \\
\hline 08 & $7560(9)$ & $5812(2)$ & $5089(2)$ & $58.6(16)$ \\
\hline N3 & $8470(8)$ & $6087(2)$ & $6445(2)$ & $43.6(14)$ \\
\hline $\mathrm{N} 4$ & $7756(9)$ & $6877(2)$ & $5374(2)$ & $47.0(15)$ \\
\hline $\mathrm{C} 27$ & $9132(9)$ & $6241(2)$ & $6094(3)$ & $42.7(16)$ \\
\hline C28 & $8019(9)$ & $6286(2)$ & $5806(2)$ & $39.9(15)$ \\
\hline C29 & $6534(9)$ & $6168(2)$ & $5983(2)$ & $39.4(15)$ \\
\hline C30 & $5036(10)$ & $6153(3)$ & $5837(3)$ & $46.3(18)$ \\
\hline C31 & $3855(10)$ & $6011(3)$ & $6093(3)$ & $50.2(19)$ \\
\hline C32 & 4197(10) & $5887(3)$ & $6482(3)$ & $48.7(18)$ \\
\hline C33 & $5692(11)$ & $5897(3)$ & $6641(3)$ & $45.3(17)$ \\
\hline C34 & $6858(9)$ & $6051(2)$ & $6384(2)$ & $36.4(14)$ \\
\hline C35 & $8246(10)$ & $6451(3)$ & $5387(2)$ & $42.7(16)$ \\
\hline C36 & $7408(11)$ & $6173(3)$ & $5084(2)$ & 49.1(18) \\
\hline C37 & $6202(16)$ & $6768(4)$ & $4787(4)$ & $75(3)$ \\
\hline C38 & $7535(13)$ & $7019(3)$ & $4964(3)$ & $61(2)$ \\
\hline C39 & $8662(10)$ & $7147(2)$ & $5625(3)$ & $42.6(16)$ \\
\hline $\mathrm{C} 40$ & $7890(12)$ & $7442(3)$ & $5842(3)$ & $50.8(19)$ \\
\hline C41 & $8732(13)$ & $7702(3)$ & $6096(3)$ & $59(2)$ \\
\hline
\end{tabular}




\begin{tabular}{|c|c|c|c|c|}
\hline Atom & $\mathbf{x}$ & $\mathbf{y}$ & $\mathbf{z}$ & $U_{e q}$ \\
\hline C42 & $10321(13)$ & $7668(3)$ & $6130(3)$ & $56(2)$ \\
\hline C43 & $11107(11)$ & $7373(3)$ & $5907(3)$ & $53(2)$ \\
\hline C44 & $10270(10)$ & $7119(3)$ & $5654(3)$ & $45.8(17)$ \\
\hline $\mathrm{C} 45$ & $6132(14)$ & $7493(4)$ & $5812(5)$ & $82(4)$ \\
\hline $\mathrm{C} 46$ & $8671(9)$ & $6551(2)$ & $7124(2)$ & $39.3(15)$ \\
\hline $\mathrm{C} 47$ & $8009(11)$ & $6534(2)$ & $7501(3)$ & $46.5(18)$ \\
\hline C48 & $7514(11)$ & $6888(3)$ & $7683(2)$ & $46.9(18)$ \\
\hline C49 & 7681(11) & $7260(2)$ & $7490(3)$ & $47.6(18)$ \\
\hline $\mathrm{C} 50$ & $8377(13)$ & $7271(2)$ & $7114(3)$ & $55(2)$ \\
\hline C51 & $8835(12)$ & $6922(2)$ & $6924(3)$ & $51(2)$ \\
\hline C52 & $7125(15)$ & $7658(3)$ & $7689(3)$ & $64(3)$ \\
\hline S3 & $2782(2)$ & $3922.7(5)$ & $3013.0(5)$ & $38.4(4)$ \\
\hline 09 & $4421(6)$ & $3949.6(18)$ & $3062.0(16)$ & $42.5(12)$ \\
\hline 010 & $1866(7)$ & $4249.4(16)$ & $2858.0(17)$ & $45.7(13)$ \\
\hline 011 & $1263(7)$ & $3486(2)$ & $5179.8(18)$ & $50.4(14)$ \\
\hline 012 & $1908(8)$ & $4052.7(19)$ & $4872.5(19)$ & $54.0(15)$ \\
\hline N5 & 2121(8) & $3830.2(19)$ & $3480.4(19)$ & $38.1(13)$ \\
\hline N6 & $2424(9)$ & $3005.6(19)$ & $4547.3(19)$ & $41.7(14)$ \\
\hline C53 & $3051(9)$ & $3706(2)$ & $3808(2)$ & $39.3(15)$ \\
\hline C54 & 2131(9) & $3611(2)$ & $4125(2)$ & $34.6(13)$ \\
\hline C55 & $538(9)$ & $3658(2)$ & $3996(2)$ & $35.7(14)$ \\
\hline C56 & $-916(10)$ & $3594(3)$ & $4184(2)$ & $43.9(17)$ \\
\hline C57 & $-2243(11)$ & $3677(3)$ & $3971(3)$ & $49.5(18)$ \\
\hline C58 & $-2193(10)$ & $3808(3)$ & $3571(2)$ & $44.6(17)$ \\
\hline C59 & $-784(10)$ & $3879(2)$ & $3374(2)$ & $40.8(15)$ \\
\hline C60 & $560(9)$ & $3792(2)$ & $3596(2)$ & $36.6(14)$ \\
\hline C61 & $2687(9)$ & $3449(2)$ & $4528(2)$ & $36.9(14)$ \\
\hline C62 & 1908(9) & $3686(2)$ & $4869(2)$ & $40.3(15)$ \\
\hline C63 & $1259(13)$ & $3041(3)$ & $5199(3)$ & $58(2)$ \\
\hline C64 & $2563(11)$ & $2865(3)$ & $4964(2)$ & $47.8(19)$ \\
\hline C65 & $3381(14)$ & $2795(2)$ & $4257(3)$ & $54.3(19)$ \\
\hline C66 & $2670(20)$ & $2566(3)$ & $3954(3)$ & $76(3)$ \\
\hline C67 & $3590(20)$ & $2388(3)$ & $3662(4)$ & $91(3)$ \\
\hline C68 & $5170(20)$ & $2435(4)$ & $3672(4)$ & $98(3)$ \\
\hline C69 & $5930(20)$ & $2652(4)$ & $3977(4)$ & $89(3)$ \\
\hline $\mathrm{C} 70$ & 4993(15) & $2828(3)$ & $4272(3)$ & $66(2)$ \\
\hline C71 & $890(20)$ & $2516(4)$ & $3950(5)$ & $98(4)$ \\
\hline C72 & $2368(9)$ & $3484(2)$ & $2733(2)$ & $37.9(14)$ \\
\hline C73 & $3121(12)$ & $3117(2)$ & $2826(3)$ & $48.3(19)$ \\
\hline C74 & $2818(14)$ & 2781(3) & $2585(3)$ & $57(2)$ \\
\hline C75 & $1847(13)$ & $2807(3)$ & $2253(3)$ & $56(2)$ \\
\hline C76 & $1111(13)$ & $3181(3)$ & $2168(3)$ & $56(2)$ \\
\hline C77 & $1373(12)$ & $3513(3)$ & $2406(2)$ & $49.6(19)$ \\
\hline C78 & $1563(18)$ & $2442(3)$ & $1987(3)$ & $76(3)$ \\
\hline S4 & $4422(2)$ & $6331.7(5)$ & $3188.7(6)$ & $41.7(4)$ \\
\hline 013 & $3943(8)$ & 6708.1(16) & $3012.6(18)$ & $50.9(15)$ \\
\hline 014 & $6022(7)$ & $6261(2)$ & $3287(2)$ & $54.7(15)$ \\
\hline 015 & $1343(9)$ & $5841(2)$ & $5234(2)$ & $62.1(17)$ \\
\hline 016 & $2367(8)$ & $6412(2)$ & $5020(2)$ & $56.3(15)$ \\
\hline N7 & $3495(7)$ & $6290.2(19)$ & $3632(2)$ & $38.1(13)$ \\
\hline N8 & $2812(9)$ & $5397(2)$ & $4613(2)$ & $45.6(15)$ \\
\hline C79 & $4122(9)$ & $6106(2)$ & $3980(2)$ & $41.1(15)$ \\
\hline C80 & $2985(10)$ & $6039(2)$ & $4246(2)$ & $39.4(15)$ \\
\hline C81 & $1543(10)$ & $6173(2)$ & $4074(2)$ & $39.6(15)$ \\
\hline C82 & $-1(10)$ & $6177(3)$ & $4202(3)$ & $47.9(19)$ \\
\hline C83 & $-1131(11)$ & $6343(3)$ & $3953(3)$ & $57(2)$ \\
\hline C84 & $-735(11)$ & $6503(3)$ & $3570(3)$ & $52.1(19)$ \\
\hline C85 & $784(10)$ & $6497(2)$ & $3431(3)$ & $44.0(16)$ \\
\hline
\end{tabular}




\begin{tabular}{lcccc}
\hline Atom & $\mathbf{x}$ & $\mathbf{y}$ & $\mathbf{z}$ & $\boldsymbol{U}_{\boldsymbol{e q}}$ \\
\hline C86 & $1880(9)$ & $6329(2)$ & $3688(2)$ & $38.7(15)$ \\
C87 & $3191(10)$ & $5833(2)$ & $4651(2)$ & $42.1(16)$ \\
C88 & $2258(10)$ & $6050(3)$ & $4977(2)$ & $47.0(17)$ \\
C89 & $1106(16)$ & $5408(3)$ & $5183(4)$ & $75(3)$ \\
C90 & $2489(13)$ & $5206(3)$ & $4998(3)$ & $62(2)$ \\
C91 & $3920(14)$ & $5182(3)$ & $4361(3)$ & $55.0(19)$ \\
C92 & $3374(19)$ & $4922(3)$ & $4062(3)$ & $72(3)$ \\
C93 & $4550(20)$ & $4735(3)$ & $3808(3)$ & $79(3)$ \\
C94 & $6090(20)$ & $4797(4)$ & $3857(4)$ & $87(3)$ \\
C95 & $6586(18)$ & $5053(3)$ & $4156(4)$ & $77(3)$ \\
C96 & $5503(14)$ & $5235(3)$ & $4407(3)$ & $60(2)$ \\
C97 & $1670(20)$ & $4848(4)$ & $4010(5)$ & $105(5)$ \\
C98 & $3698(10)$ & $5930(2)$ & $2897(2)$ & $39.6(15)$ \\
C99 & $3918(13)$ & $5528(2)$ & $3027(3)$ & $53(2)$ \\
C100 & $3370(13)$ & $5217(2)$ & $2784(3)$ & $53(2)$ \\
C101 & $2604(12)$ & $5289(2)$ & $2420(3)$ & $47.8(19)$ \\
C102 & $2418(11)$ & $5696(3)$ & $2298(3)$ & $47.1(18)$ \\
C103 & $2977(11)$ & $6010(2)$ & $2539(3)$ & $46.6(18)$ \\
C104 & $1997(14)$ & $4945(3)$ & $2160(3)$ & $61(2)$
\end{tabular}

Table 8: Anisotropic Displacement Parameters $\left(\times 10^{4}\right)$ for yp-6-98. The anisotropic displacement factor exponent takes the form: $-2 \pi^{2}\left[h^{2} a^{* 2} \times U_{11}+\ldots+2 h k a^{*} \times b^{*} \times U_{12}\right]$

\begin{tabular}{|c|c|c|c|c|c|c|}
\hline Atom & $U_{11}$ & $U_{22}$ & $\boldsymbol{U}_{33}$ & $\boldsymbol{U}_{23}$ & $U_{13}$ & $U_{12}$ \\
\hline$\overline{\mathrm{S} 1}$ & $43.2(9)$ & $36.3(8)$ & $36.7(9)$ & $3.4(7)$ & $0.1(7)$ & $3.0(7)$ \\
\hline 01 & $65(4)$ & $44(3)$ & $41(3)$ & $9(2)$ & $4(3)$ & $-1(3)$ \\
\hline 02 & $37(3)$ & $66(4)$ & $40(3)$ & $-8(3)$ & $-1(2)$ & $7(3)$ \\
\hline 03 & $62(4)$ & $48(3)$ & $39(3)$ & $-5(2)$ & $-6(3)$ & $-10(3)$ \\
\hline 04 & $72(4)$ & $39(3)$ & $50(3)$ & $-11(2)$ & $-4(3)$ & $-2(3)$ \\
\hline N1 & $43(3)$ & $38(3)$ & $38(3)$ & $0(2)$ & $4(3)$ & $-2(3)$ \\
\hline $\mathrm{N} 2$ & $46(3)$ & $34(3)$ & $38(3)$ & $3(2)$ & $1(3)$ & $-3(2)$ \\
\hline $\mathrm{C} 1$ & $36(3)$ & $36(3)$ & $38(3)$ & $2(3)$ & $5(3)$ & $4(3)$ \\
\hline $\mathrm{C} 2$ & $42(3)$ & $35(3)$ & $34(3)$ & $-2(3)$ & $1(3)$ & $-4(3)$ \\
\hline $\mathrm{C} 3$ & $39(3)$ & $33(3)$ & $41(3)$ & $-6(3)$ & $-4(3)$ & $-8(3)$ \\
\hline $\mathrm{C} 4$ & $42(4)$ & $51(4)$ & $38(4)$ & $-1(3)$ & $-3(3)$ & $-2(3)$ \\
\hline $\mathrm{C} 5$ & $38(4)$ & $65(5)$ & $49(4)$ & $-12(4)$ & $-4(3)$ & $-6(4)$ \\
\hline $\mathrm{C} 6$ & $37(4)$ & $56(5)$ & $48(4)$ & $-6(4)$ & $9(3)$ & $-9(3)$ \\
\hline $\mathrm{C} 7$ & $41(4)$ & $40(4)$ & $45(4)$ & $-5(3)$ & $7(3)$ & $-10(3)$ \\
\hline $\mathrm{C} 8$ & $34(3)$ & $32(3)$ & $42(3)$ & $-6(3)$ & $4(3)$ & $-2(3)$ \\
\hline C9 & $41(4)$ & $33(3)$ & $38(3)$ & $-5(3)$ & $-1(3)$ & $0(3)$ \\
\hline C10 & $46(4)$ & $42(4)$ & $39(4)$ & $-4(3)$ & $5(3)$ & $-5(3)$ \\
\hline C11 & $69(6)$ & $51(4)$ & $46(5)$ & $10(4)$ & $-13(4)$ & $-8(4)$ \\
\hline C12 & $59(5)$ & $49(4)$ & $38(4)$ & $4(3)$ & $-7(3)$ & $-4(4)$ \\
\hline C13 & $49(4)$ & $32(3)$ & $40(4)$ & $2(3)$ & $-8(3)$ & $-3(3)$ \\
\hline C14 & $65(5)$ & $35(4)$ & $64(5)$ & $-10(3)$ & $-7(4)$ & $7(4)$ \\
\hline C15 & $86(6)$ & $41(5)$ & $69(6)$ & $-15(4)$ & $-18(5)$ & $7(4)$ \\
\hline C16 & $82(6)$ & $40(4)$ & $64(6)$ & $-7(4)$ & $-22(5)$ & $-6(4)$ \\
\hline C17 & $57(5)$ & $50(5)$ & $71(6)$ & $4(4)$ & $-15(4)$ & $-9(4)$ \\
\hline C18 & $46(4)$ & $47(4)$ & $53(5)$ & $-5(4)$ & $-1(4)$ & $-7(3)$ \\
\hline C19 & $66(6)$ & $75(8)$ & $131(12)$ & $-46(8)$ & $-18(7)$ & $23(6)$ \\
\hline $\mathrm{C} 20$ & $42(4)$ & $39(3)$ & $37(4)$ & $4(3)$ & $-7(3)$ & $3(3)$ \\
\hline $\mathrm{C} 21$ & $58(5)$ & $30(3)$ & $61(5)$ & $-5(3)$ & $7(4)$ & $1(3)$ \\
\hline $\mathrm{C} 22$ & $80(7)$ & $33(4)$ & $65(5)$ & $-7(4)$ & $-6(5)$ & $10(4)$ \\
\hline $\mathrm{C} 23$ & $59(5)$ & $59(5)$ & $52(5)$ & $-13(4)$ & $-20(4)$ & $5(4)$ \\
\hline $\mathrm{C} 24$ & $55(5)$ & $78(5)$ & $48(5)$ & $-13(4)$ & $2(4)$ & $12(4)$ \\
\hline $\mathrm{C} 25$ & $53(5)$ & $64(5)$ & $39(4)$ & $2(3)$ & $1(3)$ & $1(4)$ \\
\hline
\end{tabular}




\begin{tabular}{|c|c|c|c|c|c|c|}
\hline Atom & $U_{11}$ & $U_{22}$ & $U_{33}$ & $U_{23}$ & $U_{13}$ & $U_{12}$ \\
\hline$\overline{\mathrm{C} 26}$ & $97(9)$ & $67(6)$ & $72(7)$ & $-27(5)$ & $-16(7)$ & $25(6)$ \\
\hline S2 & $49.7(10)$ & $35.0(9)$ & $46.2(10)$ & $-7.6(7)$ & $-11.6(8)$ & $8.9(8)$ \\
\hline 05 & $79(4)$ & $35(3)$ & $56(4)$ & $2(2)$ & $-16(3)$ & $5(3)$ \\
\hline 06 & $41(3)$ & $78(5)$ & $68(4)$ & $-22(3)$ & $-16(3)$ & $16(3)$ \\
\hline 07 & $73(4)$ & $71(4)$ & $43(3)$ & $5(3)$ & $-10(3)$ & $-23(3)$ \\
\hline 08 & $68(4)$ & $52(3)$ & $56(4)$ & $-15(3)$ & $2(3)$ & $-12(3)$ \\
\hline N3 & $42(3)$ & $46(4)$ & $43(3)$ & $-8(3)$ & $-5(3)$ & $4(3)$ \\
\hline $\mathrm{N} 4$ & $48(4)$ & $51(4)$ & $42(3)$ & $3(3)$ & $0(3)$ & $-2(3)$ \\
\hline $\mathrm{C} 27$ & $38(3)$ & $39(4)$ & $51(4)$ & $-7(3)$ & $1(3)$ & $2(3)$ \\
\hline C28 & $40(4)$ & $39(4)$ & $41(3)$ & $-5(3)$ & $4(3)$ & $1(3)$ \\
\hline C29 & $37(3)$ & $39(4)$ & $42(4)$ & $-4(3)$ & $1(3)$ & $1(3)$ \\
\hline C30 & $42(4)$ & $57(5)$ & $40(4)$ & $2(3)$ & $0(3)$ & $5(3)$ \\
\hline C31 & $38(4)$ & $57(5)$ & $55(5)$ & $0(4)$ & $2(3)$ & $-2(3)$ \\
\hline C32 & $41(4)$ & $56(5)$ & $49(4)$ & $-1(4)$ & $13(3)$ & $-5(4)$ \\
\hline C33 & $51(4)$ & $45(4)$ & $40(4)$ & $3(3)$ & $5(3)$ & $0(4)$ \\
\hline C34 & $37(3)$ & $30(3)$ & $43(4)$ & $-3(3)$ & $3(3)$ & $5(3)$ \\
\hline C35 & $43(4)$ & $46(4)$ & $39(4)$ & $-7(3)$ & $5(3)$ & $-3(3)$ \\
\hline C36 & $50(4)$ & $61(4)$ & $37(4)$ & $1(3)$ & $5(3)$ & $-15(4)$ \\
\hline C37 & $88(8)$ & $80(6)$ & $57(6)$ & $17(5)$ & $-28(6)$ & $-21(6)$ \\
\hline C38 & $70(6)$ & $68(6)$ & $44(4)$ & $12(4)$ & $-7(4)$ & $-19(5)$ \\
\hline C39 & $46(4)$ & $38(4)$ & $44(4)$ & $2(3)$ & $3(3)$ & $-1(3)$ \\
\hline $\mathrm{C} 40$ & $57(5)$ & $42(4)$ & $53(5)$ & $0(3)$ & $2(4)$ & $11(4)$ \\
\hline C41 & $78(5)$ & $38(4)$ & $62(5)$ & $-8(4)$ & $5(5)$ & $8(4)$ \\
\hline C42 & $77(5)$ & $37(4)$ & $54(5)$ & $0(3)$ & $-3(4)$ & $-8(4)$ \\
\hline C43 & $51(5)$ & $42(4)$ & $66(5)$ & $-1(4)$ & $-1(4)$ & $-4(3)$ \\
\hline C44 & $45(4)$ & $42(4)$ & $50(4)$ & $-1(3)$ & $6(3)$ & $-1(3)$ \\
\hline $\mathrm{C} 45$ & $58(6)$ & $86(8)$ & $102(10)$ & $-13(7)$ & $-3(6)$ & $24(6)$ \\
\hline $\mathrm{C} 46$ & $47(4)$ & $30(3)$ & $41(4)$ & $-1(3)$ & $-7(3)$ & $0(3)$ \\
\hline $\mathrm{C} 47$ & $57(5)$ & $36(4)$ & $47(4)$ & $3(3)$ & $-1(4)$ & $-1(3)$ \\
\hline C48 & $61(5)$ & $43(4)$ & $36(4)$ & $-3(3)$ & $5(4)$ & $3(4)$ \\
\hline C49 & $59(5)$ & $35(4)$ & $50(4)$ & $0(3)$ & $-6(4)$ & $-1(3)$ \\
\hline C50 & $83(6)$ & $25(3)$ & $58(5)$ & $2(3)$ & $8(4)$ & $3(4)$ \\
\hline C51 & $73(6)$ & $33(4)$ & $47(4)$ & $1(3)$ & $14(4)$ & $-2(4)$ \\
\hline C52 & $91(7)$ & $44(5)$ & $57(5)$ & $-8(4)$ & $-1(5)$ & $13(5)$ \\
\hline S3 & $43.4(9)$ & $35.8(8)$ & $36.0(8)$ & $3.5(7)$ & $-0.6(7)$ & $-5.6(7)$ \\
\hline 09 & $34(2)$ & $55(3)$ & $39(3)$ & $-11(2)$ & $3(2)$ & $-6(2)$ \\
\hline 010 & $55(3)$ & $36(3)$ & $47(3)$ & $7(2)$ & $0(3)$ & $0(2)$ \\
\hline 011 & $50(3)$ & $60(3)$ & $41(3)$ & $1(3)$ & $7(2)$ & $5(3)$ \\
\hline 012 & $66(4)$ & $50(3)$ & $46(3)$ & $-10(2)$ & $7(3)$ & $4(3)$ \\
\hline N5 & $40(3)$ & $39(3)$ & $35(3)$ & $4(2)$ & $-3(3)$ & $-1(3)$ \\
\hline N6 & $52(4)$ & $37(3)$ & $36(3)$ & $1(2)$ & $1(3)$ & $-2(3)$ \\
\hline C53 & $37(3)$ & $42(4)$ & $40(4)$ & $-2(3)$ & $-3(3)$ & $-4(3)$ \\
\hline C54 & $42(3)$ & $27(3)$ & $35(3)$ & $1(2)$ & $-2(3)$ & $-4(3)$ \\
\hline C55 & $40(3)$ & $32(3)$ & $35(3)$ & $-2(3)$ & $-1(3)$ & $-1(3)$ \\
\hline C56 & $46(4)$ & $46(4)$ & $40(4)$ & $-1(3)$ & $3(3)$ & $1(3)$ \\
\hline C57 & $41(4)$ & $64(5)$ & $44(4)$ & $-2(4)$ & $5(3)$ & $-2(4)$ \\
\hline C58 & $37(3)$ & $56(5)$ & $40(4)$ & $-6(3)$ & $-10(3)$ & $4(3)$ \\
\hline C59 & $45(4)$ & $41(4)$ & $37(4)$ & $-3(3)$ & $-5(3)$ & $4(3)$ \\
\hline C60 & $37(3)$ & $36(3)$ & $37(3)$ & $-3(3)$ & $-2(3)$ & $-2(3)$ \\
\hline C61 & $39(4)$ & $37(3)$ & $35(3)$ & $0(3)$ & $-1(3)$ & $0(3)$ \\
\hline C62 & $40(4)$ & $46(4)$ & $35(3)$ & $-2(3)$ & $-3(3)$ & $3(3)$ \\
\hline C63 & $65(6)$ & $59(5)$ & $49(5)$ & $8(4)$ & $14(4)$ & $-3(4)$ \\
\hline C64 & $58(5)$ & $47(4)$ & $38(4)$ & $8(3)$ & $1(3)$ & $4(4)$ \\
\hline C65 & $88(5)$ & $31(3)$ & $44(4)$ & $6(3)$ & $13(4)$ & $6(4)$ \\
\hline C66 & $140(7)$ & $32(4)$ & $56(5)$ & $0(3)$ & $-1(5)$ & $-2(5)$ \\
\hline C67 & $176(9)$ & $34(4)$ & $64(5)$ & $-11(4)$ & $11(7)$ & $5(6)$ \\
\hline C68 & $167(9)$ & $50(5)$ & $76(6)$ & $0(4)$ & $42(7)$ & $25(6)$ \\
\hline C69 & $124(8)$ & $51(5)$ & $92(6)$ & $8(5)$ & $48(6)$ & $20(5)$ \\
\hline
\end{tabular}




\begin{tabular}{|c|c|c|c|c|c|c|}
\hline Atom & $U_{11}$ & $U_{22}$ & $U_{33}$ & $U_{23}$ & $U_{13}$ & $U_{12}$ \\
\hline C70 & $88(5)$ & $40(4)$ & $69(5)$ & $7(4)$ & $28(5)$ & $17(4)$ \\
\hline C71 & $143(9)$ & $54(6)$ & $97(10)$ & $-13(6)$ & $-42(9)$ & $-21(7)$ \\
\hline C72 & $42(4)$ & $36(3)$ & $36(3)$ & $4(3)$ & $5(3)$ & $-5(3)$ \\
\hline C73 & $67(5)$ & $26(3)$ & $53(4)$ & $0(3)$ & $-7(4)$ & $-3(3)$ \\
\hline C74 & $82(6)$ & $30(4)$ & $58(5)$ & $1(3)$ & $-1(4)$ & $2(4)$ \\
\hline C75 & $79(6)$ & $49(4)$ & $41(4)$ & $-4(3)$ & $11(4)$ & $-9(4)$ \\
\hline C76 & $70(6)$ & $54(5)$ & $45(4)$ & $-4(4)$ & $-7(4)$ & $-4(4)$ \\
\hline C77 & $62(5)$ & $50(4)$ & $37(4)$ & $3(3)$ & $-4(3)$ & $5(4)$ \\
\hline C78 & $113(10)$ & $63(6)$ & $52(5)$ & $-16(5)$ & $18(6)$ & $-26(6)$ \\
\hline S4 & $46.8(10)$ & $32.3(8)$ & $45.9(10)$ & $-3.9(7)$ & $6.8(8)$ & $-7.1(7)$ \\
\hline 013 & $76(4)$ & $28(2)$ & $48(3)$ & $3(2)$ & $7(3)$ & $-13(3)$ \\
\hline 014 & $41(3)$ & $61(4)$ & $61(4)$ & $-6(3)$ & $9(3)$ & $-12(3)$ \\
\hline 015 & $71(4)$ & $67(4)$ & $48(3)$ & $12(3)$ & $15(3)$ & $13(3)$ \\
\hline 016 & $57(4)$ & $53(3)$ & $59(4)$ & $-16(3)$ & $-1(3)$ & $10(3)$ \\
\hline N7 & $36(3)$ & $33(3)$ & $45(3)$ & $2(2)$ & $0(3)$ & $-2(2)$ \\
\hline N8 & $54(4)$ & $40(3)$ & $43(3)$ & $1(3)$ & $-7(3)$ & $-5(3)$ \\
\hline C79 & $41(4)$ & $36(4)$ & $46(4)$ & $-4(3)$ & $-1(3)$ & $-3(3)$ \\
\hline C80 & $44(4)$ & $38(4)$ & $36(3)$ & $-4(3)$ & $-3(3)$ & $-1(3)$ \\
\hline C81 & $41(3)$ & $37(4)$ & $41(4)$ & $-1(3)$ & $-2(3)$ & $-3(3)$ \\
\hline C82 & $42(4)$ & $60(5)$ & $42(4)$ & $4(4)$ & $2(3)$ & $-4(3)$ \\
\hline C83 & $42(4)$ & $79(7)$ & $51(5)$ & $2(4)$ & $-3(4)$ & $-4(4)$ \\
\hline C84 & $45(4)$ & $66(5)$ & $46(4)$ & $6(4)$ & $-10(3)$ & $-1(4)$ \\
\hline C85 & $43(4)$ & $42(4)$ & $47(4)$ & $0(3)$ & $-6(3)$ & $-5(3)$ \\
\hline C86 & $38(3)$ & $35(3)$ & $43(4)$ & $-4(3)$ & $0(3)$ & $-2(3)$ \\
\hline C87 & $43(4)$ & $44(4)$ & $39(4)$ & $-4(3)$ & $-2(3)$ & $4(3)$ \\
\hline C88 & $45(4)$ & $55(4)$ & $41(4)$ & $-4(3)$ & $-5(3)$ & $9(3)$ \\
\hline C89 & $80(7)$ & $64(5)$ & $80(7)$ & $26(5)$ & $25(6)$ & $13(5)$ \\
\hline C90 & $63(6)$ & $68(6)$ & $54(5)$ & $18(4)$ & $6(4)$ & $9(5)$ \\
\hline C91 & $89(5)$ & $34(3)$ & $42(4)$ & $1(3)$ & $3(4)$ & $3(4)$ \\
\hline C92 & $125(7)$ & $42(4)$ & $50(5)$ & $0(3)$ & $-10(5)$ & $-5(5)$ \\
\hline C93 & $158(8)$ & $34(4)$ & $44(4)$ & $-1(3)$ & $1(6)$ & $3(5)$ \\
\hline C94 & $143(8)$ & $55(5)$ & $61(5)$ & $8(4)$ & $26(6)$ & $21(6)$ \\
\hline C95 & $106(7)$ & $54(5)$ & $71(6)$ & $7(4)$ & $22(5)$ & $16(5)$ \\
\hline C96 & $81(5)$ & $42(4)$ & $57(5)$ & $-2(3)$ & $8(4)$ & $9(4)$ \\
\hline C97 & $146(10)$ & $70(8)$ & $99(10)$ & $2(7)$ & $-52(9)$ & $-31(8)$ \\
\hline C98 & $48(4)$ & $29(3)$ & $42(4)$ & $1(3)$ & $9(3)$ & $-6(3)$ \\
\hline C99 & $75(6)$ & $32(4)$ & $50(5)$ & $-4(3)$ & $-7(4)$ & $-4(4)$ \\
\hline C100 & $82(6)$ & $23(3)$ & $54(4)$ & $2(3)$ & $4(4)$ & $-7(3)$ \\
\hline C101 & $63(5)$ & $33(3)$ & $48(4)$ & $1(3)$ & $13(4)$ & $0(3)$ \\
\hline C102 & $57(5)$ & $43(4)$ & $42(4)$ & $-2(3)$ & $1(4)$ & $-7(3)$ \\
\hline C103 & $54(5)$ & $30(3)$ & $56(4)$ & $2(3)$ & $-1(4)$ & $-2(3)$ \\
\hline C104 & $82(7)$ & $47(5)$ & $53(5)$ & $-2(4)$ & $6(5)$ & $-15(5)$ \\
\hline
\end{tabular}

Table 9: Bond Lengths in $\AA$ for yp-6-98.

\begin{tabular}{lll}
\hline Atom & Atom & Length/Å \\
\hline S1 & O1 & $1.427(6)$ \\
S1 & O2 & $1.420(6)$ \\
S1 & N1 & $1.660(7)$ \\
S1 & C20 & $1.754(8)$ \\
O3 & C10 & $1.328(11)$ \\
O3 & C11 & $1.442(11)$ \\
O4 & C10 & $1.209(10)$ \\
N1 & C1 & $1.400(9)$ \\
N1 & C8 & $1.404(10)$ \\
N2 & C9 & $1.472(9)$
\end{tabular}

\begin{tabular}{|c|c|c|}
\hline Atom & Atom & Length/Å \\
\hline N2 & C12 & $1.451(10)$ \\
\hline $\mathrm{N} 2$ & C13 & $1.445(10)$ \\
\hline $\mathrm{C} 1$ & $\mathrm{C} 2$ & $1.371(11)$ \\
\hline $\mathrm{C} 2$ & C3 & $1.437(11)$ \\
\hline $\mathrm{C} 2$ & $\mathrm{C} 9$ & $1.497(10)$ \\
\hline $\mathrm{C} 3$ & $\mathrm{C} 4$ & $1.404(11)$ \\
\hline $\mathrm{C} 3$ & $\mathrm{C} 8$ & $1.395(11)$ \\
\hline $\mathrm{C} 4$ & C5 & $1.385(12)$ \\
\hline $\mathrm{C} 5$ & C6 & $1.396(13)$ \\
\hline C6 & $\mathrm{C} 7$ & $1.383(12)$ \\
\hline
\end{tabular}




\begin{tabular}{|c|c|c|}
\hline Atom & Atom & Length/Å \\
\hline$\overline{\mathrm{C} 7}$ & C8 & $1.391(11)$ \\
\hline C9 & C10 & $1.528(10)$ \\
\hline C11 & $\mathrm{C} 12$ & $1.506(13)$ \\
\hline C13 & C14 & $1.409(12)$ \\
\hline C13 & C18 & $1.376(12)$ \\
\hline C14 & C15 & $1.403(14)$ \\
\hline C14 & C19 & $1.489(16)$ \\
\hline C15 & C16 & $1.381(17)$ \\
\hline C16 & $\mathrm{C} 17$ & $1.386(16)$ \\
\hline C17 & C18 & $1.382(13)$ \\
\hline $\mathrm{C} 20$ & $\mathrm{C} 21$ & $1.391(12)$ \\
\hline $\mathrm{C} 20$ & $\mathrm{C} 25$ & $1.379(12)$ \\
\hline C21 & $\mathrm{C} 22$ & $1.359(12)$ \\
\hline C22 & $\mathrm{C} 23$ & $1.383(16)$ \\
\hline C23 & $\mathrm{C} 24$ & $1.402(16)$ \\
\hline C23 & $\mathrm{C} 26$ & $1.522(13)$ \\
\hline $\mathrm{C} 24$ & $\mathrm{C} 25$ & $1.379(14)$ \\
\hline S2 & 05 & $1.420(7)$ \\
\hline S2 & 06 & $1.433(7)$ \\
\hline S2 & N3 & $1.670(7)$ \\
\hline S2 & $\mathrm{C} 46$ & $1.752(8)$ \\
\hline 07 & C36 & $1.345(12)$ \\
\hline 07 & C37 & $1.427(15)$ \\
\hline 08 & C36 & $1.189(12)$ \\
\hline N3 & $\mathrm{C} 27$ & $1.387(11)$ \\
\hline N3 & C34 & $1.403(10)$ \\
\hline $\mathrm{N} 4$ & C35 & $1.459(11)$ \\
\hline $\mathrm{N} 4$ & C38 & $1.443(11)$ \\
\hline $\mathrm{N} 4$ & C39 & $1.441(11)$ \\
\hline C27 & $\mathrm{C} 28$ & $1.359(12)$ \\
\hline C28 & $\mathrm{C} 29$ & $1.455(11)$ \\
\hline C28 & C35 & $1.499(11)$ \\
\hline C29 & C30 & $1.375(12)$ \\
\hline C29 & C34 & $1.408(11)$ \\
\hline C30 & C31 & $1.401(12)$ \\
\hline C31 & C32 & $1.382(13)$ \\
\hline C32 & C33 & $1.387(13)$ \\
\hline C33 & C34 & $1.406(11)$ \\
\hline C35 & C36 & $1.534(11)$ \\
\hline C37 & C38 & $1.525(15)$ \\
\hline C39 & $\mathrm{C} 40$ & $1.374(12)$ \\
\hline C39 & $\mathrm{C} 44$ & $1.387(12)$ \\
\hline $\mathrm{C} 40$ & $\mathrm{C} 41$ & $1.398(14)$ \\
\hline C40 & $\mathrm{C} 45$ & $1.522(15)$ \\
\hline C41 & $\mathrm{C} 42$ & $1.373(15)$ \\
\hline $\mathrm{C} 42$ & $\mathrm{C} 43$ & $1.392(14)$ \\
\hline C43 & $\mathrm{C} 44$ & $1.380(13)$ \\
\hline C46 & $\mathrm{C} 47$ & $1.371(12)$ \\
\hline C46 & C51 & $1.391(11)$ \\
\hline $\mathrm{C} 47$ & $\mathrm{C} 48$ & $1.376(12)$ \\
\hline C48 & $\mathrm{C} 49$ & $1.383(12)$ \\
\hline C49 & $\mathrm{C} 50$ & $1.382(13)$ \\
\hline C49 & C52 & $1.536(12)$ \\
\hline C50 & C51 & $1.364(12)$ \\
\hline S3 & 09 & $1.419(6)$ \\
\hline S3 & 010 & $1.424(6)$ \\
\hline S3 & N5 & $1.674(6)$ \\
\hline S3 & C72 & $1.746(8)$ \\
\hline
\end{tabular}

\begin{tabular}{|c|c|c|}
\hline Atom & Atom & Length/Å \\
\hline$\overline{011}$ & C62 & $1.338(10)$ \\
\hline 011 & C63 & $1.461(12)$ \\
\hline 012 & C62 & $1.202(10)$ \\
\hline N5 & C53 & $1.405(10)$ \\
\hline N5 & C60 & $1.399(10)$ \\
\hline N6 & C61 & $1.473(9)$ \\
\hline N6 & C64 & $1.457(10)$ \\
\hline N6 & C65 & $1.439(11)$ \\
\hline C53 & C54 & $1.351(11)$ \\
\hline C54 & C55 & $1.440(11)$ \\
\hline C54 & C61 & $1.511(10)$ \\
\hline C55 & C56 & $1.410(11)$ \\
\hline C55 & $\mathrm{C} 60$ & $1.396(10)$ \\
\hline C56 & $\mathrm{C} 57$ & $1.367(12)$ \\
\hline C57 & C58 & $1.392(12)$ \\
\hline C58 & C59 & $1.393(12)$ \\
\hline C59 & $\mathrm{C} 60$ & $1.396(11)$ \\
\hline C61 & C62 & $1.522(10)$ \\
\hline C63 & C64 & $1.481(13)$ \\
\hline C65 & C66 & $1.393(16)$ \\
\hline C65 & $\mathrm{C} 70$ & $1.388(17)$ \\
\hline C66 & C67 & $1.377(19)$ \\
\hline C66 & C71 & $1.54(2)$ \\
\hline C67 & C68 & $1.37(2)$ \\
\hline C68 & C69 & $1.40(2)$ \\
\hline C69 & $\mathrm{C} 70$ & $1.391(15)$ \\
\hline C72 & $\mathrm{C} 73$ & $1.400(11)$ \\
\hline C72 & C77 & $1.381(12)$ \\
\hline C73 & C74 & $1.384(12)$ \\
\hline C74 & $\mathrm{C} 75$ & $1.382(15)$ \\
\hline C75 & C76 & $1.408(14)$ \\
\hline C75 & $\mathrm{C} 78$ & $1.506(13)$ \\
\hline C76 & C77 & $1.362(13)$ \\
\hline S4 & 013 & $1.425(6)$ \\
\hline S4 & 014 & $1.430(7)$ \\
\hline S4 & N7 & $1.673(7)$ \\
\hline S4 & C98 & $1.746(8)$ \\
\hline 015 & C88 & $1.345(12)$ \\
\hline 015 & C89 & $1.446(14)$ \\
\hline 016 & C88 & $1.199(11)$ \\
\hline N7 & C79 & $1.406(10)$ \\
\hline N7 & C86 & $1.404(10)$ \\
\hline N8 & C87 & $1.470(10)$ \\
\hline N8 & C90 & $1.445(11)$ \\
\hline N8 & C91 & $1.449(13)$ \\
\hline C79 & $\mathrm{C} 80$ & $1.333(12)$ \\
\hline C80 & C81 & $1.431(11)$ \\
\hline C80 & C87 & $1.511(11)$ \\
\hline C81 & $\mathrm{C} 82$ & $1.391(12)$ \\
\hline C81 & C86 & $1.406(11)$ \\
\hline C82 & C83 & $1.384(13)$ \\
\hline C83 & C84 & $1.413(13)$ \\
\hline C84 & C85 & $1.382(13)$ \\
\hline C85 & C86 & $1.382(12)$ \\
\hline C87 & C88 & $1.519(11)$ \\
\hline C89 & C90 & $1.490(15)$ \\
\hline C91 & C92 & $1.384(14)$ \\
\hline C91 & C96 & $1.379(17)$ \\
\hline
\end{tabular}




\begin{tabular}{lll}
\hline Atom & Atom & Length/Å \\
\hline C92 & C93 & $1.45(2)$ \\
C92 & C97 & $1.50(2)$ \\
C93 & C94 & $1.35(2)$ \\
C94 & C95 & $1.36(2)$ \\
C95 & C96 & $1.383(16)$ \\
C98 & C99 & $1.399(11)$
\end{tabular}

\begin{tabular}{lll}
\hline Atom & Atom & Length/Å \\
\hline C98 & C103 & $1.361(12)$ \\
C99 & C100 & $1.381(12)$ \\
C100 & C101 & $1.391(14)$ \\
C101 & C102 & $1.402(11)$ \\
C101 & C104 & $1.512(12)$ \\
C102 & C103 & $1.387(12)$
\end{tabular}

Table 10: Bond Angles in ${ }^{\circ}$ for yp-6-98.

\begin{tabular}{|c|c|c|c|}
\hline Atom & Atom & Atom & Angle $/^{\circ}$ \\
\hline$\overline{01}$ & S1 & N1 & $106.8(4)$ \\
\hline 01 & $\mathrm{~S} 1$ & $\mathrm{C} 20$ & $108.9(4)$ \\
\hline 02 & $\mathrm{~S} 1$ & 01 & $121.2(4)$ \\
\hline 02 & $\mathrm{~S} 1$ & N1 & $104.4(3)$ \\
\hline 02 & $\mathrm{~S} 1$ & $\mathrm{C} 20$ & $108.9(4)$ \\
\hline N1 & $\mathrm{S} 1$ & $\mathrm{C} 20$ & $105.4(3)$ \\
\hline C10 & 03 & C11 & $121.1(6)$ \\
\hline $\mathrm{C} 1$ & N1 & $\mathrm{S} 1$ & $124.0(6)$ \\
\hline $\mathrm{C} 1$ & $\mathrm{~N} 1$ & $\mathrm{C} 8$ & $108.5(6)$ \\
\hline $\mathrm{C} 8$ & N1 & $\mathrm{S} 1$ & $126.2(5)$ \\
\hline C12 & N2 & $\mathrm{C} 9$ & $111.5(6)$ \\
\hline C13 & N2 & C9 & $112.8(6)$ \\
\hline C13 & N2 & C12 & $114.2(6)$ \\
\hline $\mathrm{C} 2$ & $\mathrm{C} 1$ & N1 & $109.5(7)$ \\
\hline $\mathrm{C} 1$ & $\mathrm{C} 2$ & $\mathrm{C} 3$ & $106.5(6)$ \\
\hline $\mathrm{C} 1$ & $\mathrm{C} 2$ & $\mathrm{C} 9$ & $125.6(7)$ \\
\hline $\mathrm{C} 3$ & $\mathrm{C} 2$ & $\mathrm{C} 9$ & $127.9(7)$ \\
\hline $\mathrm{C} 4$ & C3 & $\mathrm{C} 2$ & $132.3(7)$ \\
\hline $\mathrm{C} 8$ & $\mathrm{C} 3$ & $\mathrm{C} 2$ & $108.8(7)$ \\
\hline $\mathrm{C} 8$ & $\mathrm{C} 3$ & $\mathrm{C} 4$ & $118.9(7)$ \\
\hline $\mathrm{C} 5$ & $\mathrm{C} 4$ & $\mathrm{C} 3$ & $117.6(7)$ \\
\hline $\mathrm{C} 4$ & C5 & C6 & $122.4(8)$ \\
\hline $\mathrm{C} 7$ & C6 & C5 & $120.7(8)$ \\
\hline $\mathrm{C} 6$ & $\mathrm{C} 7$ & $\mathrm{C} 8$ & $116.7(7)$ \\
\hline $\mathrm{C} 3$ & $\mathrm{C} 8$ & N1 & $106.8(6)$ \\
\hline $\mathrm{C} 7$ & $\mathrm{C} 8$ & N1 & $129.6(7)$ \\
\hline $\mathrm{C} 7$ & $\mathrm{C} 8$ & $\mathrm{C} 3$ & $123.6(8)$ \\
\hline N2 & $\mathrm{C} 9$ & $\mathrm{C} 2$ & $108.7(6)$ \\
\hline N2 & $\mathrm{C} 9$ & C10 & $112.6(6)$ \\
\hline $\mathrm{C} 2$ & C9 & C10 & $110.1(6)$ \\
\hline 03 & C10 & $\mathrm{C} 9$ & $121.3(7)$ \\
\hline 04 & C10 & 03 & $118.0(7)$ \\
\hline 04 & C10 & $\mathrm{C} 9$ & $120.5(8)$ \\
\hline 03 & C11 & $\mathrm{C} 12$ & $111.7(8)$ \\
\hline N2 & C12 & C11 & $106.8(7)$ \\
\hline C14 & C13 & N2 & $117.0(8)$ \\
\hline C18 & C13 & N2 & $122.5(8)$ \\
\hline C18 & C13 & C14 & $120.6(8)$ \\
\hline C13 & C14 & C19 & $121.5(9)$ \\
\hline C15 & C14 & C13 & $118.2(10)$ \\
\hline C15 & C14 & C19 & $120.3(10)$ \\
\hline C16 & C15 & C14 & $120.2(10)$ \\
\hline C15 & C16 & C17 & $121.0(9)$ \\
\hline C18 & C17 & C16 & $119.3(10)$ \\
\hline C13 & C18 & C17 & $120.7(9)$ \\
\hline $\mathrm{C} 21$ & $\mathrm{C} 20$ & S1 & $120.5(6)$ \\
\hline
\end{tabular}

\begin{tabular}{|c|c|c|c|}
\hline Atom & Atom & Atom & Angle $/^{\circ}$ \\
\hline$\overline{\mathrm{C} 25}$ & $\mathrm{C} 20$ & S1 & $118.8(7)$ \\
\hline $\mathrm{C} 25$ & $\mathrm{C} 20$ & $\mathrm{C} 21$ & $120.7(8)$ \\
\hline $\mathrm{C} 22$ & $\mathrm{C} 21$ & $\mathrm{C} 20$ & $120.0(9)$ \\
\hline $\mathrm{C} 21$ & $\mathrm{C} 22$ & $\mathrm{C} 23$ & $120.6(10)$ \\
\hline $\mathrm{C} 22$ & $\mathrm{C} 23$ & $\mathrm{C} 24$ & $119.3(9)$ \\
\hline $\mathrm{C} 22$ & $\mathrm{C} 23$ & $\mathrm{C} 26$ & $120.0(11)$ \\
\hline $\mathrm{C} 24$ & $\mathrm{C} 23$ & $\mathrm{C} 26$ & $120.7(11)$ \\
\hline $\mathrm{C} 25$ & $\mathrm{C} 24$ & $\mathrm{C} 23$ & $120.2(9)$ \\
\hline $\mathrm{C} 20$ & $\mathrm{C} 25$ & $\mathrm{C} 24$ & $119.2(9)$ \\
\hline 05 & $\mathrm{~S} 2$ & 06 & $120.5(4)$ \\
\hline 05 & $\mathrm{~S} 2$ & N3 & $107.1(4)$ \\
\hline 05 & $\mathrm{~S} 2$ & C46 & $108.7(4)$ \\
\hline 06 & $\mathrm{~S} 2$ & N3 & $104.0(4)$ \\
\hline 06 & $\mathrm{~S} 2$ & C46 & $110.3(4)$ \\
\hline N3 & $\mathrm{S} 2$ & C46 & $105.0(4)$ \\
\hline C36 & 07 & C37 & $121.2(8)$ \\
\hline $\mathrm{C} 27$ & N3 & $\mathrm{S} 2$ & $123.8(6)$ \\
\hline $\mathrm{C} 27$ & N3 & C34 & $108.3(7)$ \\
\hline C34 & N3 & $\mathrm{S} 2$ & $124.8(6)$ \\
\hline C38 & $\mathrm{N} 4$ & C35 & $111.9(7)$ \\
\hline C39 & N4 & C35 & $114.5(7)$ \\
\hline C39 & $\mathrm{N} 4$ & C38 & $114.5(7)$ \\
\hline $\mathrm{C} 28$ & $\mathrm{C} 27$ & N3 & $109.8(7)$ \\
\hline $\mathrm{C} 27$ & $\mathrm{C} 28$ & C29 & $107.7(7)$ \\
\hline $\mathrm{C} 27$ & $\mathrm{C} 28$ & C35 & $126.6(8)$ \\
\hline $\mathrm{C} 29$ & $\mathrm{C} 28$ & C35 & $125.6(7)$ \\
\hline $\mathrm{C} 30$ & $\mathrm{C} 29$ & $\mathrm{C} 28$ & $133.3(8)$ \\
\hline $\mathrm{C} 30$ & $\mathrm{C} 29$ & C34 & $120.5(7)$ \\
\hline C34 & $\mathrm{C} 29$ & $\mathrm{C} 28$ & $106.2(7)$ \\
\hline $\mathrm{C} 29$ & C30 & C31 & $118.4(8)$ \\
\hline $\mathrm{C} 32$ & C31 & C30 & $120.5(8)$ \\
\hline C31 & C32 & C33 & $122.8(8)$ \\
\hline C32 & C33 & C34 & 116.1(8) \\
\hline N3 & C34 & $\mathrm{C} 29$ & $107.9(7)$ \\
\hline N3 & C34 & C33 & $130.2(8)$ \\
\hline C33 & C34 & $\mathrm{C} 29$ & $121.7(7)$ \\
\hline N4 & C35 & $\mathrm{C} 28$ & $109.6(6)$ \\
\hline N4 & C35 & C36 & $114.5(7)$ \\
\hline $\mathrm{C} 28$ & C35 & C36 & $109.1(7)$ \\
\hline 07 & C36 & C35 & $119.3(8)$ \\
\hline 08 & C36 & 07 & $118.7(8)$ \\
\hline 08 & C36 & C35 & $122.0(8)$ \\
\hline 07 & C37 & C38 & $112.2(10)$ \\
\hline N4 & C38 & C37 & $106.6(8)$ \\
\hline $\mathrm{C} 40$ & C39 & N4 & $118.2(8)$ \\
\hline $\mathrm{C} 40$ & C39 & C44 & 119.3(9) \\
\hline
\end{tabular}




\begin{tabular}{|c|c|c|c|}
\hline Atom & Atom & Atom & Angle $/^{\circ}$ \\
\hline$\overline{\mathrm{C} 44}$ & C39 & $\mathrm{N} 4$ & $122.4(8)$ \\
\hline C39 & $\mathrm{C} 40$ & C41 & $119.6(9)$ \\
\hline C39 & $\mathrm{C} 40$ & $\mathrm{C} 45$ & $121.4(10)$ \\
\hline C41 & $\mathrm{C} 40$ & $\mathrm{C} 45$ & $119.0(9)$ \\
\hline $\mathrm{C} 42$ & $\mathrm{C} 41$ & $\mathrm{C} 40$ & $120.8(9)$ \\
\hline C41 & $\mathrm{C} 42$ & $\mathrm{C} 43$ & $119.7(9)$ \\
\hline C44 & $\mathrm{C} 43$ & $\mathrm{C} 42$ & $119.2(9)$ \\
\hline C43 & $\mathrm{C} 44$ & C39 & $121.4(8)$ \\
\hline C47 & C46 & $\mathrm{S} 2$ & $119.4(6)$ \\
\hline C47 & $\mathrm{C} 46$ & C51 & $120.6(7)$ \\
\hline C51 & $\mathrm{C} 46$ & $\mathrm{~S} 2$ & $120.0(6)$ \\
\hline C46 & $\mathrm{C} 47$ & $\mathrm{C} 48$ & $119.4(8)$ \\
\hline C47 & $\mathrm{C} 48$ & C49 & $120.8(8)$ \\
\hline C48 & $\mathrm{C} 49$ & C52 & $121.3(8)$ \\
\hline C50 & $\mathrm{C} 49$ & $\mathrm{C} 48$ & $118.9(8)$ \\
\hline C50 & $\mathrm{C} 49$ & C52 & $119.8(8)$ \\
\hline C51 & $\mathrm{C} 50$ & $\mathrm{C} 49$ & $121.1(8)$ \\
\hline C50 & C51 & $\mathrm{C} 46$ & $119.2(8)$ \\
\hline 09 & S3 & 010 & $122.8(4)$ \\
\hline 09 & S3 & N5 & $104.0(3)$ \\
\hline 09 & S3 & $\mathrm{C} 72$ & $108.3(4)$ \\
\hline 010 & S3 & N5 & $106.3(3)$ \\
\hline 010 & S3 & $\mathrm{C} 72$ & $108.4(4)$ \\
\hline N5 & S3 & $\mathrm{C} 72$ & $105.8(3)$ \\
\hline C62 & 011 & C63 & $121.6(7)$ \\
\hline C53 & N5 & S3 & $124.8(6)$ \\
\hline C60 & N5 & S3 & $126.3(5)$ \\
\hline C60 & N5 & C53 & $108.0(6)$ \\
\hline C64 & N6 & C61 & $109.9(6)$ \\
\hline C65 & N6 & C61 & $110.9(6)$ \\
\hline C65 & N6 & C64 & $115.6(7)$ \\
\hline C54 & C53 & N5 & $109.5(7)$ \\
\hline C53 & C54 & C55 & $107.5(6)$ \\
\hline C53 & C54 & C61 & $125.6(7)$ \\
\hline C55 & C54 & C61 & $126.7(7)$ \\
\hline C56 & C55 & $\mathrm{C} 54$ & $133.9(7)$ \\
\hline C60 & C55 & C54 & $107.6(7)$ \\
\hline C60 & C55 & C56 & $118.5(7)$ \\
\hline C57 & C56 & C55 & $118.7(7)$ \\
\hline C56 & C57 & C58 & $121.7(8)$ \\
\hline C57 & C58 & C59 & $121.5(8)$ \\
\hline C58 & C59 & C60 & $116.0(7)$ \\
\hline C55 & $\mathrm{C} 60$ & N5 & $107.4(7)$ \\
\hline C59 & $\mathrm{C} 60$ & N5 & $129.1(7)$ \\
\hline C59 & $\mathrm{C} 60$ & $\mathrm{C} 55$ & $123.4(8)$ \\
\hline N6 & C61 & C54 & $109.6(6)$ \\
\hline N6 & C61 & C62 & $113.8(6)$ \\
\hline C54 & C61 & C62 & $109.6(6)$ \\
\hline 011 & C62 & C61 & $120.1(7)$ \\
\hline 012 & C62 & 011 & $118.8(7)$ \\
\hline 012 & C62 & C61 & $121.1(7)$ \\
\hline 011 & C63 & C64 & $111.3(7)$ \\
\hline N6 & C64 & C63 & $108.2(7)$ \\
\hline C66 & C65 & N6 & $119.1(11)$ \\
\hline C70 & C65 & N6 & $120.5(9)$ \\
\hline C70 & C65 & C66 & $120.4(11)$ \\
\hline C65 & C66 & C71 & $120.0(12)$ \\
\hline C67 & C66 & C65 & $118.7(15)$ \\
\hline
\end{tabular}

\begin{tabular}{|c|c|c|c|}
\hline Atom & Atom & Atom & Angle ${ }^{\circ}$ \\
\hline$\overline{C 67}$ & C66 & C71 & $121.3(13)$ \\
\hline C68 & C67 & C66 & $120.4(14)$ \\
\hline C67 & C68 & C69 & $122.7(13)$ \\
\hline $\mathrm{C} 70$ & C69 & C68 & $116.3(16)$ \\
\hline C65 & $\mathrm{C} 70$ & C69 & $121.4(13)$ \\
\hline C73 & $\mathrm{C} 72$ & S3 & $119.8(6)$ \\
\hline C77 & $\mathrm{C} 72$ & S3 & $119.0(6)$ \\
\hline C77 & $\mathrm{C} 72$ & C73 & 121.1(8) \\
\hline C74 & C73 & $\mathrm{C} 72$ & $118.2(8)$ \\
\hline C75 & C74 & C73 & $121.4(9)$ \\
\hline C74 & C75 & C76 & $118.9(8)$ \\
\hline C74 & $\mathrm{C} 75$ & C78 & $120.9(10)$ \\
\hline C76 & $\mathrm{C} 75$ & C78 & $120.1(10)$ \\
\hline C77 & C76 & $\mathrm{C} 75$ & $120.4(9)$ \\
\hline C76 & $\mathrm{C} 77$ & $\mathrm{C} 72$ & $120.0(9)$ \\
\hline 013 & S4 & 014 & $120.6(4)$ \\
\hline 013 & S4 & N7 & $106.9(4)$ \\
\hline 013 & S4 & C98 & $108.9(4)$ \\
\hline 014 & S4 & N7 & $104.1(4)$ \\
\hline 014 & S4 & C98 & $110.3(4)$ \\
\hline N7 & S4 & C98 & $104.7(3)$ \\
\hline C88 & 015 & C89 & $120.6(8)$ \\
\hline C79 & N7 & $\mathrm{S} 4$ & $124.7(5)$ \\
\hline C86 & N7 & $\mathrm{S} 4$ & $125.3(6)$ \\
\hline C86 & N7 & C79 & $108.0(6)$ \\
\hline C90 & N8 & C87 & $112.8(7)$ \\
\hline C90 & N8 & C91 & $115.0(7)$ \\
\hline C91 & N8 & $\mathrm{C} 87$ & $112.2(7)$ \\
\hline C80 & C79 & N7 & $109.3(7)$ \\
\hline C79 & $\mathrm{C} 80$ & C81 & $108.7(7)$ \\
\hline C79 & $\mathrm{C} 80$ & C87 & $125.0(8)$ \\
\hline C81 & $\mathrm{C} 80$ & C87 & $126.2(7)$ \\
\hline C82 & C81 & $\mathrm{C} 80$ & $134.9(8)$ \\
\hline C82 & C81 & C86 & $118.0(8)$ \\
\hline C86 & C81 & $\mathrm{C} 80$ & 107.1(7) \\
\hline C83 & C82 & C81 & $119.3(8)$ \\
\hline C82 & C83 & C84 & $120.8(9)$ \\
\hline C85 & $\mathrm{C} 84$ & C83 & $121.3(8)$ \\
\hline C86 & $\mathrm{C} 85$ & $\mathrm{C} 84$ & $116.3(8)$ \\
\hline N7 & C86 & C81 & $106.9(7)$ \\
\hline C85 & C86 & N7 & $128.8(8)$ \\
\hline C85 & C86 & C81 & $124.3(8)$ \\
\hline N8 & $\mathrm{C} 87$ & $\mathrm{C} 80$ & $109.5(6)$ \\
\hline N8 & C87 & C88 & $113.6(7)$ \\
\hline C80 & C87 & C88 & $110.9(7)$ \\
\hline 015 & C88 & C87 & $121.2(8)$ \\
\hline 016 & C88 & 015 & $118.3(8)$ \\
\hline 016 & C88 & C87 & $120.5(8)$ \\
\hline 015 & C89 & C90 & $111.8(10)$ \\
\hline N8 & C90 & C89 & $108.8(8)$ \\
\hline C92 & C91 & N8 & $119.2(11)$ \\
\hline C96 & C91 & N8 & $121.4(9)$ \\
\hline C96 & C91 & C92 & $119.4(11)$ \\
\hline C91 & C92 & C93 & $116.1(13)$ \\
\hline C91 & C92 & C97 & $121.0(13)$ \\
\hline C93 & C92 & C97 & $123.0(12)$ \\
\hline C94 & C93 & C92 & $123.3(11)$ \\
\hline C93 & C94 & C95 & $118.9(13)$ \\
\hline
\end{tabular}




\begin{tabular}{llll}
\hline Atom & Atom & Atom & \multicolumn{1}{c}{ Angle $^{\circ}$} \\
\hline C94 & C95 & C96 & $119.6(15)$ \\
C91 & C96 & C95 & $122.8(11)$ \\
C99 & C98 & S4 & $119.5(7)$ \\
C103 & C98 & S4 & $119.9(6)$ \\
C103 & C98 & C99 & $120.6(7)$ \\
C100 & C99 & C98 & $118.2(8)$
\end{tabular}

\begin{tabular}{llll}
\hline Atom & Atom & Atom & Angle $^{\circ}$ \\
\hline C99 & C100 & C101 & $122.5(8)$ \\
C100 & C101 & C102 & $117.7(8)$ \\
C100 & C101 & C104 & $121.9(8)$ \\
C102 & C101 & C104 & $120.4(8)$ \\
C103 & C102 & C101 & $120.0(8)$ \\
C98 & C103 & C102 & $121.0(7)$
\end{tabular}

Table 11: Torsion Angles in ${ }^{\circ}$ for yp-6-98.

\begin{tabular}{|c|c|c|c|c|}
\hline Atom & Atom & Atom & Atom & Angle $/^{\circ}$ \\
\hline$\overline{\mathrm{S} 1}$ & N1 & $\mathrm{C} 1$ & $\mathrm{C} 2$ & $-168.2(5)$ \\
\hline S1 & N1 & $\mathrm{C} 8$ & $\mathrm{C} 3$ & $167.9(5)$ \\
\hline $\mathrm{S} 1$ & N1 & $\mathrm{C} 8$ & $\mathrm{C} 7$ & $-14.3(11)$ \\
\hline $\mathrm{S} 1$ & $\mathrm{C} 20$ & $\mathrm{C} 21$ & $\mathrm{C} 22$ & $-177.8(8)$ \\
\hline $\mathrm{S} 1$ & $\mathrm{C} 20$ & $\mathrm{C} 25$ & $\mathrm{C} 24$ & $177.6(7)$ \\
\hline 01 & $\mathrm{~S} 1$ & N1 & $\mathrm{C} 1$ & $-150.1(6)$ \\
\hline 01 & $\mathrm{~S} 1$ & N1 & $\mathrm{C} 8$ & $44.6(7)$ \\
\hline 01 & $\mathrm{~S} 1$ & $\mathrm{C} 20$ & $\mathrm{C} 21$ & $-177.4(7)$ \\
\hline 01 & $\mathrm{~S} 1$ & $\mathrm{C} 20$ & $\mathrm{C} 25$ & $4.2(8)$ \\
\hline 02 & $\mathrm{~S} 1$ & N1 & $\mathrm{C} 1$ & $-20.6(7)$ \\
\hline 02 & $\mathrm{~S} 1$ & N1 & $\mathrm{C} 8$ & $174.2(6)$ \\
\hline 02 & $\mathrm{~S} 1$ & $\mathrm{C} 20$ & $\mathrm{C} 21$ & $48.4(8)$ \\
\hline 02 & $\mathrm{~S} 1$ & $\mathrm{C} 20$ & $\mathrm{C} 25$ & $-130.0(7)$ \\
\hline 03 & $\mathrm{C} 11$ & $\mathrm{C} 12$ & $\mathrm{~N} 2$ & $-58.4(10)$ \\
\hline N1 & $\mathrm{S} 1$ & $\mathrm{C} 20$ & $\mathrm{C} 21$ & $-63.1(8)$ \\
\hline N1 & $\mathrm{S} 1$ & $\mathrm{C} 20$ & $\mathrm{C} 25$ & $118.5(7)$ \\
\hline N1 & $\mathrm{C} 1$ & $\mathrm{C} 2$ & $\mathrm{C} 3$ & $0.4(8)$ \\
\hline N1 & $\mathrm{C} 1$ & $\mathrm{C} 2$ & C9 & $178.2(7)$ \\
\hline N2 & $\mathrm{C} 9$ & C10 & 03 & $6.4(11)$ \\
\hline $\mathrm{N} 2$ & $\mathrm{C} 9$ & C10 & 04 & $-176.9(8)$ \\
\hline $\mathrm{N} 2$ & $\mathrm{C} 13$ & C14 & C15 & $178.0(8)$ \\
\hline $\mathrm{N} 2$ & $\mathrm{C} 13$ & C14 & C19 & $-2.1(15)$ \\
\hline $\mathrm{N} 2$ & $\mathrm{C} 13$ & C18 & $\mathrm{C} 17$ & $-177.3(8)$ \\
\hline $\mathrm{C} 1$ & N1 & C8 & $\mathrm{C} 3$ & $0.8(8)$ \\
\hline $\mathrm{C} 1$ & N1 & C8 & $\mathrm{C} 7$ & $178.5(7)$ \\
\hline $\mathrm{C} 1$ & $\mathrm{C} 2$ & $\mathrm{C} 3$ & $\mathrm{C} 4$ & $-179.4(8)$ \\
\hline $\mathrm{C} 1$ & $\mathrm{C} 2$ & $\mathrm{C} 3$ & $\mathrm{C} 8$ & $0.0(8)$ \\
\hline $\mathrm{C} 1$ & $\mathrm{C} 2$ & $\mathrm{C} 9$ & $\mathrm{~N} 2$ & $-101.8(9)$ \\
\hline $\mathrm{C} 1$ & $\mathrm{C} 2$ & $\mathrm{C} 9$ & C10 & $134.5(8)$ \\
\hline $\mathrm{C} 2$ & $\mathrm{C} 3$ & $\mathrm{C} 4$ & $\mathrm{C} 5$ & $178.3(8)$ \\
\hline $\mathrm{C} 2$ & $\mathrm{C} 3$ & $\mathrm{C} 8$ & N1 & $-0.5(8)$ \\
\hline $\mathrm{C} 2$ & $\mathrm{C} 3$ & $\mathrm{C} 8$ & $\mathrm{C} 7$ & $-178.4(7)$ \\
\hline $\mathrm{C} 2$ & $\mathrm{C} 9$ & C10 & 03 & $127.8(8)$ \\
\hline $\mathrm{C} 2$ & $\mathrm{C} 9$ & C10 & 04 & $-55.5(10)$ \\
\hline $\mathrm{C} 3$ & $\mathrm{C} 2$ & $\mathrm{C} 9$ & $\mathrm{~N} 2$ & $75.5(9)$ \\
\hline $\mathrm{C} 3$ & $\mathrm{C} 2$ & $\mathrm{C} 9$ & C10 & $-48.3(10)$ \\
\hline $\mathrm{C} 3$ & $\mathrm{C} 4$ & $\mathrm{C} 5$ & $\mathrm{C} 6$ & $1.2(13)$ \\
\hline $\mathrm{C} 4$ & $\mathrm{C} 3$ & $\mathrm{C} 8$ & $\mathrm{~N} 1$ & $179.1(7)$ \\
\hline $\mathrm{C} 4$ & $\mathrm{C} 3$ & $\mathrm{C} 8$ & $\mathrm{C} 7$ & $1.1(11)$ \\
\hline $\mathrm{C} 4$ & $\mathrm{C} 5$ & C6 & $\mathrm{C} 7$ & $-1.1(14)$ \\
\hline $\mathrm{C} 5$ & $\mathrm{C} 6$ & $\mathrm{C} 7$ & $\mathrm{C} 8$ & $0.9(12)$ \\
\hline $\mathrm{C} 6$ & $\mathrm{C} 7$ & $\mathrm{C} 8$ & N1 & $-178.4(8)$ \\
\hline $\mathrm{C} 6$ & $\mathrm{C} 7$ & C8 & $\mathrm{C} 3$ & $-1.0(11)$ \\
\hline $\mathrm{C} 8$ & N1 & $\mathrm{C} 1$ & $\mathrm{C} 2$ & $-0.8(8)$ \\
\hline $\mathrm{C} 8$ & $\mathrm{C} 3$ & $\mathrm{C} 4$ & $\mathrm{C} 5$ & $-1.2(11)$ \\
\hline $\mathrm{C} 9$ & $\mathrm{~N} 2$ & C12 & C11 & $66.0(9)$ \\
\hline
\end{tabular}




\begin{tabular}{|c|c|c|c|c|}
\hline Atom & Atom & Atom & Atom & Angle $/^{\circ}$ \\
\hline$\overline{\mathrm{C} 9}$ & N2 & C13 & C14 & $-129.7(8)$ \\
\hline $\mathrm{C} 9$ & $\mathrm{~N} 2$ & C13 & C18 & $50.0(10)$ \\
\hline $\mathrm{C} 9$ & $\mathrm{C} 2$ & $\mathrm{C} 3$ & $\mathrm{C} 4$ & $2.9(13)$ \\
\hline $\mathrm{C} 9$ & $\mathrm{C} 2$ & $\mathrm{C} 3$ & $\mathrm{C} 8$ & $-177.6(7)$ \\
\hline C10 & 03 & C11 & C12 & $26.3(12)$ \\
\hline C11 & 03 & C10 & 04 & $-176.8(8)$ \\
\hline C11 & 03 & C10 & $\mathrm{C} 9$ & $-0.1(12)$ \\
\hline $\mathrm{C} 12$ & $\mathrm{~N} 2$ & $\mathrm{C} 9$ & $\mathrm{C} 2$ & $-162.5(7)$ \\
\hline $\mathrm{C} 12$ & $\mathrm{~N} 2$ & $\mathrm{C} 9$ & C10 & $-40.2(9)$ \\
\hline $\mathrm{C} 12$ & $\mathrm{~N} 2$ & C13 & C14 & $101.6(9)$ \\
\hline C12 & $\mathrm{N} 2$ & C13 & C18 & $-78.6(10)$ \\
\hline C13 & $\mathrm{N} 2$ & $\mathrm{C} 9$ & $\mathrm{C} 2$ & $67.5(8)$ \\
\hline C13 & $\mathrm{N} 2$ & $\mathrm{C} 9$ & C10 & $-170.2(7)$ \\
\hline C13 & $\mathrm{N} 2$ & C12 & C11 & $-164.7(7)$ \\
\hline C13 & C14 & C15 & C16 & $-0.2(16)$ \\
\hline C14 & $\mathrm{C} 13$ & C18 & C17 & $2.4(14)$ \\
\hline C14 & C15 & C16 & C17 & $1.7(18)$ \\
\hline C15 & $\mathrm{C} 16$ & C17 & C18 & $-1.1(17)$ \\
\hline C16 & C17 & C18 & C13 & $-1.0(15)$ \\
\hline C18 & C13 & C14 & C15 & $-1.8(14)$ \\
\hline C18 & C13 & C14 & C19 & $178.1(11)$ \\
\hline C19 & $\mathrm{C} 14$ & C15 & C16 & $179.9(12)$ \\
\hline $\mathrm{C} 20$ & $\mathrm{~S} 1$ & $\mathrm{~N} 1$ & $\mathrm{C} 1$ & $94.1(7)$ \\
\hline $\mathrm{C} 20$ & $\mathrm{~S} 1$ & $\mathrm{~N} 1$ & $\mathrm{C} 8$ & $-71.1(7)$ \\
\hline $\mathrm{C} 20$ & $\mathrm{C} 21$ & C22 & $\mathrm{C} 23$ & $0.3(16)$ \\
\hline $\mathrm{C} 21$ & $\mathrm{C} 20$ & $\mathrm{C} 25$ & $\mathrm{C} 24$ & $-0.8(13)$ \\
\hline $\mathrm{C} 21$ & $\mathrm{C} 22$ & C23 & $\mathrm{C} 24$ & $-1.0(16)$ \\
\hline $\mathrm{C} 21$ & $\mathrm{C} 22$ & C23 & $\mathrm{C} 26$ & $178.9(10)$ \\
\hline $\mathrm{C} 22$ & C23 & C24 & $\mathrm{C} 25$ & $0.8(15)$ \\
\hline $\mathrm{C} 23$ & $\mathrm{C} 24$ & $\mathrm{C} 25$ & $\mathrm{C} 20$ & $0.1(14)$ \\
\hline $\mathrm{C} 25$ & $\mathrm{C} 20$ & C21 & $\mathrm{C} 22$ & $0.6(14)$ \\
\hline $\mathrm{C} 26$ & $\mathrm{C} 23$ & C24 & $\mathrm{C} 25$ & $-179.1(10)$ \\
\hline $\mathrm{S} 2$ & N3 & C27 & $\mathrm{C} 28$ & $-163.8(6)$ \\
\hline $\mathrm{S} 2$ & N3 & C34 & $\mathrm{C} 29$ & $163.2(6)$ \\
\hline S2 & N3 & C34 & C33 & $-22.2(12)$ \\
\hline $\mathrm{S} 2$ & $\mathrm{C} 46$ & C47 & $\mathrm{C} 48$ & $178.7(7)$ \\
\hline $\mathrm{S} 2$ & $\mathrm{C} 46$ & C51 & C50 & $-176.9(8)$ \\
\hline 05 & $\mathrm{~S} 2$ & N3 & $\mathrm{C} 27$ & $-151.5(7)$ \\
\hline 05 & $\mathrm{~S} 2$ & N3 & C34 & $50.8(8)$ \\
\hline 05 & $\mathrm{~S} 2$ & C46 & $\mathrm{C} 47$ & $12.1(8)$ \\
\hline 05 & $\mathrm{~S} 2$ & C46 & C51 & $-168.8(7)$ \\
\hline 06 & $\mathrm{~S} 2$ & N3 & $\mathrm{C} 27$ & $-22.9(8)$ \\
\hline 06 & $\mathrm{~S} 2$ & N3 & C34 & $179.3(7)$ \\
\hline 06 & $\mathrm{~S} 2$ & C46 & $\mathrm{C} 47$ & $-122.0(7)$ \\
\hline 06 & $\mathrm{~S} 2$ & C46 & C51 & $57.1(8)$ \\
\hline 07 & C37 & C38 & N4 & $-58.9(13)$ \\
\hline N3 & $\mathrm{S} 2$ & C46 & $\mathrm{C} 47$ & $126.5(7)$ \\
\hline N3 & $\mathrm{S} 2$ & C46 & C51 & $-54.5(8)$ \\
\hline N3 & $\mathrm{C} 27$ & C28 & $\mathrm{C} 29$ & $2.1(9)$ \\
\hline N3 & $\mathrm{C} 27$ & C28 & $\mathrm{C} 35$ & $178.7(7)$ \\
\hline $\mathrm{N} 4$ & C35 & C36 & 07 & $11.2(11)$ \\
\hline $\mathrm{N} 4$ & C35 & C36 & 08 & $-171.7(8)$ \\
\hline $\mathrm{N} 4$ & C39 & $\mathrm{C} 40$ & C41 & $178.5(8)$ \\
\hline N4 & C39 & $\mathrm{C} 40$ & $\mathrm{C} 45$ & $-1.8(14)$ \\
\hline $\mathrm{N} 4$ & C39 & C44 & $\mathrm{C} 43$ & $-177.8(8)$ \\
\hline $\mathrm{C} 27$ & N3 & C34 & $\mathrm{C} 29$ & $2.6(9)$ \\
\hline $\mathrm{C} 27$ & N3 & C34 & C33 & $177.2(8)$ \\
\hline $\mathrm{C} 27$ & $\mathrm{C} 28$ & C29 & $\mathrm{C} 30$ & $179.9(9)$ \\
\hline
\end{tabular}




\begin{tabular}{|c|c|c|c|c|}
\hline Atom & Atom & Atom & Atom & Angle $/^{\circ}$ \\
\hline$\overline{\mathrm{C} 27}$ & $\mathrm{C} 28$ & C29 & $\mathrm{C} 34$ & $-0.4(9)$ \\
\hline $\mathrm{C} 27$ & $\mathrm{C} 28$ & C35 & $\mathrm{N} 4$ & $-99.8(9)$ \\
\hline $\mathrm{C} 27$ & C28 & C35 & C36 & $134.2(9)$ \\
\hline $\mathrm{C} 28$ & C29 & C30 & C31 & $178.2(9)$ \\
\hline $\mathrm{C} 28$ & $\mathrm{C} 29$ & C34 & N3 & $-1.3(8)$ \\
\hline $\mathrm{C} 28$ & $\mathrm{C} 29$ & C34 & C33 & $-176.5(7)$ \\
\hline $\mathrm{C} 28$ & C35 & C36 & 07 & $134.5(8)$ \\
\hline $\mathrm{C} 28$ & C35 & C36 & 08 & $-48.4(11)$ \\
\hline $\mathrm{C} 29$ & $\mathrm{C} 28$ & C35 & N4 & $76.3(10)$ \\
\hline C29 & $\mathrm{C} 28$ & C35 & C36 & $-49.8(10)$ \\
\hline $\mathrm{C} 29$ & C30 & C31 & C32 & $-0.3(14)$ \\
\hline C30 & C29 & C34 & N3 & $178.4(8)$ \\
\hline C30 & $\mathrm{C} 29$ & C34 & C33 & $3.2(12)$ \\
\hline C30 & C31 & $\mathrm{C} 32$ & C33 & $0.2(14)$ \\
\hline C31 & C32 & C33 & C34 & $1.5(13)$ \\
\hline C32 & C33 & C34 & N3 & $-177.2(8)$ \\
\hline C32 & C33 & C34 & C29 & $-3.2(12)$ \\
\hline C34 & N3 & $\mathrm{C} 27$ & C28 & $-2.9(9)$ \\
\hline C34 & C29 & C30 & C31 & $-1.4(13)$ \\
\hline C35 & $\mathrm{N} 4$ & C38 & C37 & $63.7(12)$ \\
\hline C35 & $\mathrm{N} 4$ & C39 & $\mathrm{C} 40$ & $-138.4(8)$ \\
\hline C35 & $\mathrm{N} 4$ & C39 & C44 & $41.5(11)$ \\
\hline C35 & $\mathrm{C} 28$ & C29 & C30 & $3.3(15)$ \\
\hline C35 & $\mathrm{C} 28$ & $\mathrm{C} 29$ & C34 & $-177.1(7)$ \\
\hline C36 & 07 & C37 & C38 & $31.7(14)$ \\
\hline C37 & 07 & C36 & 08 & $175.2(10)$ \\
\hline C37 & 07 & C36 & C35 & $-7.6(13)$ \\
\hline C38 & N4 & C35 & $\mathrm{C} 28$ & $-163.9(8)$ \\
\hline C38 & $\mathrm{N} 4$ & C35 & C36 & $-40.9(10)$ \\
\hline C38 & $\mathrm{N} 4$ & C39 & $\mathrm{C} 40$ & $90.5(10)$ \\
\hline C38 & $\mathrm{N} 4$ & C39 & C44 & $-89.6(11)$ \\
\hline C39 & $\mathrm{N} 4$ & C35 & C28 & $63.7(9)$ \\
\hline C39 & N4 & C35 & C36 & $-173.3(7)$ \\
\hline C39 & N4 & C38 & C37 & $-163.9(9)$ \\
\hline C39 & $\mathrm{C} 40$ & C41 & $\mathrm{C} 42$ & $0.2(15)$ \\
\hline $\mathrm{C} 40$ & C39 & C44 & $\mathrm{C} 43$ & $2.1(14)$ \\
\hline $\mathrm{C} 40$ & C41 & $\mathrm{C} 42$ & C43 & $0.4(16)$ \\
\hline C41 & C42 & $\mathrm{C} 43$ & C44 & $0.2(15)$ \\
\hline $\mathrm{C} 42$ & $\mathrm{C} 43$ & $\mathrm{C} 44$ & C39 & $-1.5(14)$ \\
\hline C44 & C39 & C40 & C41 & $-1.4(13)$ \\
\hline C44 & C39 & $\mathrm{C} 40$ & $\mathrm{C} 45$ & $178.3(10)$ \\
\hline $\mathrm{C} 45$ & $\mathrm{C} 40$ & $\mathrm{C} 41$ & C42 & $-179.6(11)$ \\
\hline C46 & $\mathrm{S} 2$ & N3 & $\mathrm{C} 27$ & $93.0(7)$ \\
\hline C46 & $\mathrm{S} 2$ & N3 & C34 & $-64.7(7)$ \\
\hline C46 & C47 & $\mathrm{C} 48$ & C49 & $-0.2(14)$ \\
\hline C47 & C46 & C51 & C50 & $2.2(15)$ \\
\hline C47 & C48 & C49 & $\mathrm{C} 50$ & $-1.0(15)$ \\
\hline C47 & C48 & $\mathrm{C} 49$ & C52 & $179.7(9)$ \\
\hline $\mathrm{C} 48$ & C49 & C50 & C51 & $2.8(16)$ \\
\hline C49 & C50 & C51 & $\mathrm{C} 46$ & $-3.4(16)$ \\
\hline C51 & $\mathrm{C} 46$ & $\mathrm{C} 47$ & C48 & $-0.4(14)$ \\
\hline C52 & C49 & C50 & C51 & $-177.8(10)$ \\
\hline S3 & N5 & C53 & C54 & $-172.6(5)$ \\
\hline S3 & N5 & C60 & C55 & $171.6(5)$ \\
\hline S3 & N5 & C60 & C59 & $-10.6(11)$ \\
\hline S3 & C72 & C73 & C74 & $-177.1(7)$ \\
\hline S3 & C72 & C77 & C76 & $175.9(8)$ \\
\hline 09 & S3 & N5 & C53 & $-14.4(7)$ \\
\hline
\end{tabular}




\begin{tabular}{|c|c|c|c|c|}
\hline Atom & Atom & Atom & Atom & Angle $/^{\circ}$ \\
\hline 09 & S3 & N5 & $\mathrm{C} 60$ & $177.7(6)$ \\
\hline 09 & S3 & C72 & $\mathrm{C} 73$ & $44.7(8)$ \\
\hline 09 & S3 & C72 & $\mathrm{C} 77$ & $-131.1(7)$ \\
\hline 010 & S3 & N5 & C53 & $-145.3(6)$ \\
\hline 010 & S3 & N5 & C60 & $46.7(7)$ \\
\hline 010 & S3 & C72 & C73 & $-179.9(7)$ \\
\hline 010 & S3 & C72 & $\mathrm{C} 77$ & $4.2(8)$ \\
\hline 011 & C63 & C64 & N6 & $-57.8(10)$ \\
\hline N5 & S3 & C72 & $\mathrm{C} 73$ & $-66.2(7)$ \\
\hline N5 & S3 & C72 & $\mathrm{C} 77$ & $117.9(7)$ \\
\hline N5 & C53 & C54 & C55 & $2.4(8)$ \\
\hline N5 & C53 & C54 & C61 & $177.5(6)$ \\
\hline N6 & C61 & C62 & 011 & $9.3(10)$ \\
\hline N6 & C61 & C62 & 012 & $-173.4(8)$ \\
\hline N6 & C65 & C66 & C67 & $175.7(9)$ \\
\hline N6 & C65 & C66 & C71 & $-4.1(14)$ \\
\hline N6 & C65 & $\mathrm{C} 70$ & C69 & $-175.2(9)$ \\
\hline C53 & N5 & C60 & C55 & $1.9(8)$ \\
\hline C53 & N5 & C60 & C59 & $179.7(8)$ \\
\hline C53 & C54 & C55 & C56 & $178.0(8)$ \\
\hline C53 & C54 & C55 & C60 & $-1.2(8)$ \\
\hline C53 & C54 & C61 & N6 & $-101.7(8)$ \\
\hline C53 & C54 & C61 & C62 & $132.7(8)$ \\
\hline $\mathrm{C} 54$ & C55 & C56 & C57 & $178.9(9)$ \\
\hline C54 & C55 & $\mathrm{C} 60$ & N5 & $-0.5(8)$ \\
\hline C54 & C55 & $\mathrm{C} 60$ & C59 & $-178.4(7)$ \\
\hline $\mathrm{C} 54$ & C61 & C62 & 011 & $132.4(7)$ \\
\hline C54 & C61 & C62 & 012 & $-50.4(10)$ \\
\hline C55 & C54 & C61 & N6 & $72.4(9)$ \\
\hline C55 & C54 & C61 & C62 & $-53.1(9)$ \\
\hline C55 & C56 & $\mathrm{C} 57$ & C58 & $2.5(13)$ \\
\hline C56 & C55 & $\mathrm{C} 60$ & N5 & $-179.8(7)$ \\
\hline C56 & C55 & $\mathrm{C} 60$ & C59 & $2.3(11)$ \\
\hline C56 & $\mathrm{C} 57$ & C58 & C59 & $-3.1(14)$ \\
\hline C57 & C58 & C59 & C60 & $3.0(12)$ \\
\hline C58 & C59 & $\mathrm{C} 60$ & N5 & $179.8(8)$ \\
\hline C58 & C59 & $\mathrm{C} 60$ & C55 & $-2.7(11)$ \\
\hline C60 & N5 & C53 & C54 & $-2.7(8)$ \\
\hline C60 & C55 & C56 & $\mathrm{C} 57$ & $-2.0(11)$ \\
\hline C61 & N6 & C64 & C63 & $66.8(9)$ \\
\hline C61 & N6 & C65 & C66 & $-119.0(8)$ \\
\hline C61 & N6 & C65 & $\mathrm{C} 70$ & $59.5(10)$ \\
\hline C61 & C54 & C55 & C56 & $2.9(13)$ \\
\hline C61 & C54 & C55 & C60 & $-176.2(7)$ \\
\hline C62 & 011 & C63 & C64 & $25.4(12)$ \\
\hline C63 & 011 & $\mathrm{C} 62$ & 012 & $-178.4(8)$ \\
\hline C63 & 011 & $\mathrm{C} 62$ & C61 & $-1.1(12)$ \\
\hline C64 & N6 & C61 & C54 & $-165.1(7)$ \\
\hline C64 & N6 & C61 & C62 & $-42.0(9)$ \\
\hline C64 & N6 & C65 & C66 & $115.0(9)$ \\
\hline C64 & N6 & C65 & $\mathrm{C} 70$ & $-66.5(11)$ \\
\hline C65 & N6 & C61 & C54 & $65.9(9)$ \\
\hline C65 & N6 & C61 & C62 & $-171.0(7)$ \\
\hline C65 & N6 & C64 & C63 & $-166.8(8)$ \\
\hline C65 & C66 & $\mathrm{C} 67$ & C68 & $0.4(18)$ \\
\hline C66 & C65 & $\mathrm{C} 70$ & C69 & $3.3(15)$ \\
\hline C66 & C67 & C68 & C69 & $2(2)$ \\
\hline C67 & C68 & C69 & $\mathrm{C} 70$ & $-1.2(19)$ \\
\hline
\end{tabular}




\begin{tabular}{|c|c|c|c|c|}
\hline Atom & Atom & Atom & Atom & Angle $/^{\circ}$ \\
\hline$\overline{\mathrm{C} 68}$ & C69 & C70 & C65 & $-1.3(16)$ \\
\hline $\mathrm{C} 70$ & $\mathrm{C} 65$ & C66 & C67 & $-2.8(15)$ \\
\hline $\mathrm{C} 70$ & C65 & C66 & C71 & $177.4(10)$ \\
\hline C71 & C66 & C67 & C68 & $-179.8(12)$ \\
\hline C72 & S3 & N5 & C53 & $99.6(7)$ \\
\hline C72 & S3 & N5 & C60 & $-68.4(7)$ \\
\hline C72 & C73 & C74 & C75 & $2.5(15)$ \\
\hline $\mathrm{C} 73$ & $\mathrm{C} 72$ & C77 & C76 & $0.0(14)$ \\
\hline C73 & C74 & $\mathrm{C} 75$ & C76 & $-2.3(16)$ \\
\hline $\mathrm{C} 73$ & C74 & $\mathrm{C} 75$ & C78 & $178.1(10)$ \\
\hline C74 & C75 & C76 & C77 & $1.0(16)$ \\
\hline C75 & C76 & C77 & C72 & $0.1(15)$ \\
\hline C77 & C72 & $\mathrm{C} 73$ & C74 & $-1.3(13)$ \\
\hline C78 & C75 & C76 & C77 & $-179.5(10)$ \\
\hline $\mathrm{S} 4$ & N7 & C79 & $\mathrm{C} 80$ & $-165.8(6)$ \\
\hline S4 & N7 & $\mathrm{C} 86$ & C81 & $165.3(5)$ \\
\hline $\mathrm{S} 4$ & N7 & $\mathrm{C} 86$ & C85 & $-16.7(12)$ \\
\hline $\mathrm{S} 4$ & C98 & C99 & $\mathrm{C} 100$ & $-178.3(8)$ \\
\hline S4 & C98 & C103 & C102 & 178.8(7) \\
\hline 013 & S4 & N7 & $\mathrm{C} 79$ & $-145.8(6)$ \\
\hline 013 & S4 & N7 & C86 & $52.3(7)$ \\
\hline 013 & S4 & C98 & C99 & $-175.2(7)$ \\
\hline 013 & S4 & C98 & C103 & $7.2(8)$ \\
\hline 014 & $\mathrm{~S} 4$ & N7 & C79 & $-17.0(7)$ \\
\hline 014 & $\mathrm{~S} 4$ & N7 & C86 & $-179.0(6)$ \\
\hline 014 & $\mathrm{~S} 4$ & C98 & C99 & $50.3(8)$ \\
\hline 014 & $\mathrm{~S} 4$ & C98 & C103 & $-127.3(7)$ \\
\hline 015 & C89 & C90 & N8 & $-57.7(13)$ \\
\hline N7 & $\mathrm{S} 4$ & C98 & C99 & $-61.2(8)$ \\
\hline N7 & S4 & C98 & C103 & $121.2(7)$ \\
\hline N7 & C79 & $\mathrm{C} 80$ & $\mathrm{C} 81$ & $1.1(9)$ \\
\hline N7 & C79 & C80 & $\mathrm{C} 87$ & $177.7(7)$ \\
\hline N8 & C87 & $\mathrm{C} 88$ & 015 & $8.3(11)$ \\
\hline N8 & C87 & C88 & 016 & $-174.5(8)$ \\
\hline N8 & C91 & C92 & C93 & $176.8(8)$ \\
\hline N8 & C91 & C92 & C97 & $-2.8(15)$ \\
\hline N8 & C91 & C96 & C95 & $-176.1(9)$ \\
\hline C79 & N7 & $\mathrm{C} 86$ & C81 & $0.8(8)$ \\
\hline C79 & N7 & $\mathrm{C} 86$ & C85 & $178.8(8)$ \\
\hline C79 & $\mathrm{C} 80$ & C81 & $\mathrm{C} 82$ & $179.1(9)$ \\
\hline C79 & $\mathrm{C} 80$ & C81 & C86 & $-0.6(9)$ \\
\hline C79 & $\mathrm{C} 80$ & C87 & N8 & $-95.5(9)$ \\
\hline C79 & $\mathrm{C} 80$ & C87 & C88 & $138.4(8)$ \\
\hline $\mathrm{C} 80$ & C81 & C82 & C83 & $178.5(9)$ \\
\hline $\mathrm{C} 80$ & $\mathrm{C} 81$ & $\mathrm{C} 86$ & N7 & $-0.2(8)$ \\
\hline $\mathrm{C} 80$ & C81 & $\mathrm{C} 86$ & $\mathrm{C} 85$ & $-178.3(7)$ \\
\hline $\mathrm{C} 80$ & C87 & C88 & 015 & $132.0(8)$ \\
\hline $\mathrm{C} 80$ & C87 & C88 & 016 & $-50.8(11)$ \\
\hline $\mathrm{C} 81$ & $\mathrm{C} 80$ & C87 & N8 & $80.5(10)$ \\
\hline C81 & $\mathrm{C} 80$ & C87 & $\mathrm{C} 88$ & $-45.5(11)$ \\
\hline C81 & $\mathrm{C} 82$ & C83 & $\mathrm{C} 84$ & $0.8(15)$ \\
\hline C82 & C81 & $\mathrm{C} 86$ & N7 & $-179.9(7)$ \\
\hline C82 & C81 & $\mathrm{C} 86$ & C85 & $2.0(12)$ \\
\hline $\mathrm{C} 82$ & $\mathrm{C} 83$ & C84 & $\mathrm{C} 85$ & $0.4(16)$ \\
\hline $\mathrm{C} 83$ & $\mathrm{C} 84$ & C85 & C86 & $-0.4(14)$ \\
\hline C84 & C85 & C86 & N7 & $-178.6(8)$ \\
\hline $\mathrm{C} 84$ & $\mathrm{C} 85$ & C86 & C81 & $-0.8(12)$ \\
\hline C86 & N7 & C79 & $\mathrm{C} 80$ & $-1.2(9)$ \\
\hline
\end{tabular}




\begin{tabular}{|c|c|c|c|c|}
\hline Atom & Atom & Atom & Atom & Angle $/^{\circ}$ \\
\hline$\overline{\mathrm{C} 86}$ & C81 & C82 & $\mathrm{C} 83$ & $-1.8(13)$ \\
\hline $\mathrm{C} 87$ & N8 & $\mathrm{C} 90$ & C89 & $61.7(12)$ \\
\hline C87 & N8 & C91 & C92 & $-132.7(9)$ \\
\hline $\mathrm{C} 87$ & N8 & C91 & C96 & $46.1(11)$ \\
\hline C87 & $\mathrm{C} 80$ & C81 & C82 & $2.5(15)$ \\
\hline C87 & $\mathrm{C} 80$ & C81 & C86 & $-177.2(7)$ \\
\hline $\mathrm{C} 88$ & 015 & C89 & $\mathrm{C} 90$ & $30.5(14)$ \\
\hline C89 & 015 & C88 & 016 & $176.8(10)$ \\
\hline C89 & 015 & C88 & C87 & $-6.0(13)$ \\
\hline $\mathrm{C} 90$ & N8 & C87 & $\mathrm{C} 80$ & $-161.3(8)$ \\
\hline $\mathrm{C} 90$ & N8 & C87 & C88 & $-36.7(10)$ \\
\hline $\mathrm{C} 90$ & N8 & C91 & C92 & $96.6(11)$ \\
\hline C90 & N8 & C91 & C96 & $-84.5(11)$ \\
\hline C91 & N8 & C87 & $\mathrm{C} 80$ & $67.0(9)$ \\
\hline C91 & N8 & C87 & C88 & $-168.5(7)$ \\
\hline C91 & N8 & $\mathrm{C} 90$ & C89 & $-167.9(10)$ \\
\hline C91 & C92 & C93 & C94 & $1.4(16)$ \\
\hline C92 & C91 & C96 & C95 & $2.8(15)$ \\
\hline C92 & C93 & C94 & C95 & $-1.1(18)$ \\
\hline C93 & C94 & C95 & C96 & $1.5(17)$ \\
\hline C94 & $\mathrm{C} 95$ & C96 & C91 & $-2.4(17)$ \\
\hline C96 & C91 & C92 & C93 & $-2.1(14)$ \\
\hline C96 & C91 & C92 & C97 & $178.3(11)$ \\
\hline C97 & $\mathrm{C} 92$ & $\mathrm{C} 93$ & C94 & $-179.1(12)$ \\
\hline C98 & S4 & N7 & C79 & $98.8(7)$ \\
\hline C98 & S4 & N7 & C86 & $-63.2(7)$ \\
\hline C98 & C99 & C100 & C101 & $-0.5(16)$ \\
\hline C99 & C98 & C103 & C102 & $1.2(14)$ \\
\hline C99 & $\mathrm{C} 100$ & C101 & C102 & $1.0(15)$ \\
\hline C99 & $\mathrm{C} 100$ & C101 & C104 & $-179.4(10)$ \\
\hline C100 & C101 & C102 & C103 & $-0.5(14)$ \\
\hline C101 & $\mathrm{C} 102$ & C103 & C98 & $-0.6(14)$ \\
\hline C103 & C98 & C99 & C100 & $-0.7(14)$ \\
\hline C104 & C101 & C102 & C103 & $179.9(9)$ \\
\hline
\end{tabular}


Table 12: Hydrogen Fractional Atomic Coordinates $\left(\times 10^{4}\right)$ and Equivalent Isotropic Displacement Parameters $\left(\AA^{2} \times 10^{3}\right)$ for yp-6-98. $U_{e q}$ is defined as $1 / 3$ of the trace of the orthogonalised $U_{i j}$.

\begin{tabular}{|c|c|c|c|c|}
\hline Atom & $\mathbf{x}$ & $\mathbf{y}$ & $\mathbf{z}$ & $U_{e q}$ \\
\hline$\overline{\mathrm{H} 1}$ & 9251.78 & 3675 & 6251.49 & 44 \\
\hline $\mathrm{H} 4$ & 4135.45 & 3791.43 & 5583.59 & 52 \\
\hline H5 & 1877.9 & 3646.84 & 5950.04 & 60 \\
\hline H6 & 1963.85 & 3474.64 & 6631.35 & 56 \\
\hline H7 & 4366.53 & 3416.62 & 6968.21 & 50 \\
\hline H9 & 8901.51 & 3810.39 & 5493.81 & 45 \\
\hline $\mathrm{H} 11 \mathrm{~A}$ & 5197.95 & 4339.27 & 4965.25 & 66 \\
\hline H11B & 6170.53 & 4309.42 & 4553.07 & 66 \\
\hline $\mathrm{H} 12 \mathrm{~A}$ & 8531.08 & 4338.08 & 4920.72 & 58 \\
\hline H12B & 7484.16 & 4730.78 & 5025.89 & 58 \\
\hline H15 & 8184.17 & 5281.08 & 6399.92 & 78 \\
\hline H16 & 10839.12 & 5155.16 & 6454.93 & 74 \\
\hline H17 & 12043.89 & 4668.44 & 6045.38 & 71 \\
\hline H18 & 10551.78 & 4291.69 & 5589.24 & 58 \\
\hline H19A & 5790.91 & 5031.39 & 5668.56 & 136 \\
\hline H19B & 5720.85 & 5122.58 & 6143.7 & 136 \\
\hline H19C & 5466.84 & 4667.75 & 5980.42 & 136 \\
\hline H21 & 8896.48 & 4338.24 & 6884.82 & 60 \\
\hline H22 & 8467.04 & 4964.78 & 7181.43 & 71 \\
\hline H24 & 5620.25 & 4434.45 & 8005.11 & 72 \\
\hline H25 & 6044.29 & 3801.06 & 7702.4 & 62 \\
\hline $\mathrm{H} 26 \mathrm{~A}$ & 7787.25 & 5305.56 & 7872.63 & 118 \\
\hline H26B & 6221.43 & 5141.12 & 8078.67 & 118 \\
\hline $\mathrm{H} 26 \mathrm{C}$ & 6157.5 & 5350.84 & 7641.9 & 118 \\
\hline H27 & 10203.93 & 6305.01 & 6060.85 & 51 \\
\hline H30 & 4807.5 & 6237.4 & 5568.13 & 56 \\
\hline H31 & 2811.62 & 5999.01 & 5998 & 60 \\
\hline H32 & 3372.85 & 5790.63 & 6648.59 & 58 \\
\hline H33 & 5915.88 & 5805.31 & 6907.12 & 54 \\
\hline H35 & 9385.25 & 6441.74 & 5324.78 & 51 \\
\hline H37A & 5242.36 & 6820.97 & 4944.95 & 90 \\
\hline H37B & 6011.38 & 6856.17 & 4505.35 & 90 \\
\hline H38A & 8497.17 & 6977.84 & 4804.22 & 73 \\
\hline H38B & 7269.45 & 7312.47 & 4962.13 & 73 \\
\hline H41 & 8197.75 & 7905.52 & 6247.31 & 71 \\
\hline H42 & 10881.79 & 7845.56 & 6304.2 & 67 \\
\hline H43 & 12205.78 & 7345.56 & 5928.16 & 64 \\
\hline H44 & 10806.09 & 6921.6 & 5496.47 & 55 \\
\hline $\mathrm{H} 45 \mathrm{~A}$ & 5848.98 & 7565.4 & 5534.21 & 123 \\
\hline H45B & 5792.87 & 7709.92 & 5996.29 & 123 \\
\hline $\mathrm{H} 45 \mathrm{C}$ & 5620.47 & 7236.41 & 5886.91 & 123 \\
\hline $\mathrm{H} 47$ & 7893.67 & 6279.2 & 7635.2 & 56 \\
\hline H48 & 7052.16 & 6877.17 & 7943.68 & 56 \\
\hline H50 & 8539.61 & 7526.88 & 6984.58 & 66 \\
\hline H51 & 9261.28 & 6931.55 & 6659.26 & 61 \\
\hline $\mathrm{H} 52 \mathrm{~A}$ & 8016.26 & 7840.88 & 7728.16 & 96 \\
\hline H52B & 6650.07 & 7596.59 & 7951.39 & 96 \\
\hline $\mathrm{H} 52 \mathrm{C}$ & 6355.5 & 7790.71 & 7513.92 & 96 \\
\hline H53 & 4156.62 & 3692.06 & 3806.12 & 47 \\
\hline H56 & -972.08 & 3494.24 & 4453.87 & 53 \\
\hline H57 & -3225.27 & 3644.37 & 4099.78 & 59 \\
\hline H58 & -3141.55 & 3849.92 & 3428.98 & 54 \\
\hline
\end{tabular}




\begin{tabular}{|c|c|c|c|c|}
\hline H59 & -740.94 & 3980.64 & 3105.49 & 49 \\
\hline H61 & 3834.76 & 3498.55 & 4546.35 & 44 \\
\hline H63A & 259.11 & 2936.35 & 5091.82 & 69 \\
\hline H63B & 1347.14 & 2953.26 & 5485.03 & 69 \\
\hline H64A & 3571.81 & 2953.66 & 5079.1 & 57 \\
\hline H64B & 2518.74 & 2563.05 & 4972.93 & 57 \\
\hline H67 & 3121.44 & 2232.11 & 3452.92 & 110 \\
\hline H68 & 5773.7 & 2315.14 & 3462.9 & 117 \\
\hline H69 & 7036.17 & 2677.89 & 3982.99 & 107 \\
\hline $\mathrm{H} 70$ & 5462.95 & 2973.65 & 4488.51 & 79 \\
\hline H71A & 559.99 & 2358.34 & 4187.26 & 147 \\
\hline H71B & 571.46 & 2372.51 & 3703.47 & 147 \\
\hline H71C & 394.28 & 2785.74 & 3957.18 & 147 \\
\hline H73 & 3821.57 & 3099.87 & 3047.39 & 58 \\
\hline H74 & 3286.6 & 2526.58 & 2650.44 & 68 \\
\hline H76 & 427.1 & 3201.8 & 1942.87 & 68 \\
\hline H77 & 870.92 & 3764.61 & 2347.08 & 60 \\
\hline H78A & 2559.86 & 2339.95 & 1883.44 & 114 \\
\hline H78B & 895.15 & 2520.77 & 1759.48 & 114 \\
\hline H78C & 1049.06 & 2226.93 & 2143.96 & 114 \\
\hline H79 & 5188.75 & 6040.48 & 4019.14 & 49 \\
\hline H82 & -277.57 & 6066.47 & 4457.91 & 57 \\
\hline H83 & -2184.54 & 6349.15 & 4040.1 & 69 \\
\hline H84 & -1528.07 & 6617.41 & 3404.24 & 63 \\
\hline H85 & 1059.56 & 6603.25 & 3173.13 & 53 \\
\hline H87 & 4316.7 & 5853.83 & 4726.06 & 50 \\
\hline H89A & 185.08 & 5361.68 & 5008.3 & 89 \\
\hline H89B & 891.48 & 5282.22 & 5449.52 & 89 \\
\hline $\mathrm{H} 90 \mathrm{~A}$ & 3401.49 & 5234.69 & 5179.05 & 74 \\
\hline H90B & 2280.32 & 4911.99 & 4958.54 & 74 \\
\hline H93 & 4212.5 & 4559.6 & 3596.2 & 94 \\
\hline H94 & 6825.46 & 4663.75 & 3686.4 & 104 \\
\hline H95 & 7665.91 & 5105.21 & 4191.28 & 92 \\
\hline H96 & 5865.17 & 5404.34 & 4620.58 & 72 \\
\hline H97A & 1306.78 & 4653.8 & 4215.77 & 157 \\
\hline H97B & 1471.1 & 4734.53 & 3740.35 & 157 \\
\hline H97C & 1098.67 & 5105.45 & 4039.91 & 157 \\
\hline H99 & 4430.37 & 5471.02 & 3275.2 & 63 \\
\hline H100 & 3520.62 & 4942.65 & 2869.3 & 64 \\
\hline H102 & 1907.92 & 5756.06 & 2050.19 & 57 \\
\hline H103 & 2853.99 & 6284.13 & 2452.52 & 56 \\
\hline $\mathrm{H} 10 \mathrm{~A}$ & 2828.43 & 4744.78 & 2115.13 & 91 \\
\hline H10B & 1647.62 & 5053.74 & 1899.48 & 91 \\
\hline $\mathrm{H} 10 \mathrm{C}$ & 1119.73 & 4811.86 & 2296.96 & 91 \\
\hline
\end{tabular}




\section{Crystallographic data for 7}

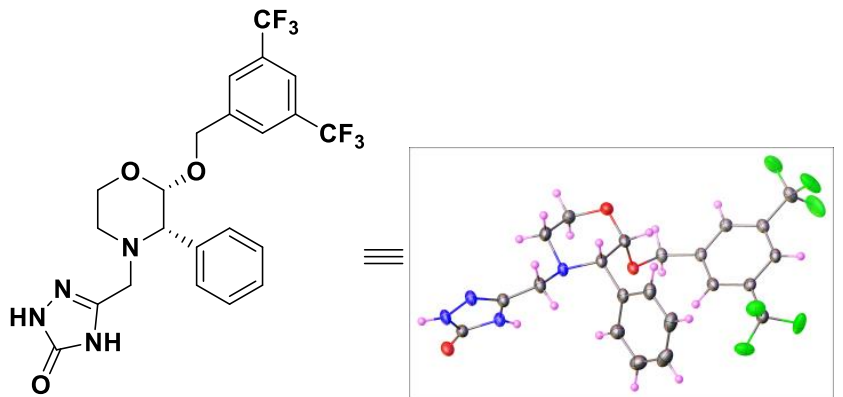

\begin{tabular}{|c|c|}
\hline Compound & YP-6-143 \\
\hline Formula & $\mathrm{C}_{22} \mathrm{H}_{20} \mathrm{~F}_{6} \mathrm{~N}_{4} \mathrm{O}_{3}$ \\
\hline$D_{\text {calc. }} / \mathrm{g} \mathrm{cm}^{-3}$ & 1.360 \\
\hline$\mu / \mathrm{mm}^{-1}$ & 1.073 \\
\hline Formula Weight & 502.42 \\
\hline Colour & colourless \\
\hline Shape & irregular \\
\hline Size $/ \mathrm{mm}^{3}$ & $0.23 \times 0.13 \times 0.12$ \\
\hline$T / \mathrm{K}$ & $140.00(10)$ \\
\hline Crystal System & monoclinic \\
\hline Space Group & $C 2 / c$ \\
\hline$a / \AA ̊$ & $17.11684(17)$ \\
\hline$b / \AA ̊$ & $28.2538(3)$ \\
\hline$c / \AA$ & $10.15151(9)$ \\
\hline$\left.\alpha\right|^{\circ}$ & 90 \\
\hline$\beta /^{\circ}$ & $91.3149(8)$ \\
\hline$\gamma /^{\circ}$ & 90 \\
\hline $\mathrm{V} / \AA^{3}$ & $4908.14(8)$ \\
\hline$Z$ & 8 \\
\hline$Z^{\prime}$ & 1 \\
\hline Wavelength/Å & 1.54184 \\
\hline Radiation type & $\mathrm{CuK \alpha}$ \\
\hline$\Theta_{\min } /^{\circ}$ & 3.019 \\
\hline$\Theta_{\max } /^{\circ}$ & 72.654 \\
\hline Measured Refl's. & 41521 \\
\hline Indep't Refl's & 4868 \\
\hline Refl's $I \geq 2 \sigma(I)$ & 4571 \\
\hline$R_{\text {int }}$ & 0.0297 \\
\hline Parameters & 325 \\
\hline Restraints & 0 \\
\hline Largest Peak/e $\AA^{-3}$ & 0.306 \\
\hline Deepest Hole/e $\AA^{-3}$ & -0.288 \\
\hline GooF & 1.033 \\
\hline$w R_{2}$ (all data) & 0.0890 \\
\hline$w R_{2}$ & 0.0873 \\
\hline$R_{1}$ (all data) & 0.0339 \\
\hline$R_{1}$ & 0.0323 \\
\hline
\end{tabular}


Table 13: Fractional Atomic Coordinates $\left(\times 10^{4}\right)$ and Equivalent Isotropic Displacement Parameters $\left(\AA^{2} \times 10^{3}\right)$ for yp-6-143. $U_{e q}$ is defined as $1 / 3$ of the trace of the orthogonalised $U_{i j}$.

\begin{tabular}{|c|c|c|c|c|}
\hline Atom & $\mathbf{x}$ & $\mathbf{y}$ & $\mathbf{z}$ & $U_{e q}$ \\
\hline$\overline{F 1}$ & $916.0(4)$ & $2232.9(3)$ & $3046.1(7)$ & $35.50(19)$ \\
\hline $\mathrm{F} 2$ & $819.7(4)$ & $2719.7(3)$ & $1443.8(8)$ & $38.4(2)$ \\
\hline F3 & $791.7(4)$ & $1975.4(3)$ & $1070.5(9)$ & $48.2(2)$ \\
\hline F4 & $3521.0(7)$ & $1294.8(3)$ & $-169.2(9)$ & $60.6(3)$ \\
\hline F5 & $4365.4(5)$ & $1437.8(3)$ & $1344.6(12)$ & $55.1(3)$ \\
\hline F6 & $3323.8(5)$ & 1059.8(3) & $1782.9(10)$ & $49.6(2)$ \\
\hline 01 & $4402.9(5)$ & $3346.3(3)$ & $4427.9(8)$ & $25.63(18)$ \\
\hline 02 & $3418.2(5)$ & $3484.9(3)$ & $2857.7(7)$ & $20.67(17)$ \\
\hline 03 & $3463.0(5)$ & $5925.0(3)$ & $4558.9(8)$ & $26.90(19)$ \\
\hline N1 & $3396.4(5)$ & $4104.5(3)$ & 5198.9(9) & $20.4(2)$ \\
\hline $\mathrm{N} 2$ & $3228.8(6)$ & $5123.1(3)$ & $5013.9(9)$ & $22.1(2)$ \\
\hline N3 & $3350.1(6)$ & $5597.3(3)$ & $6656.0(9)$ & $25.1(2)$ \\
\hline N4 & $3222.9(6)$ & $5151.9(3)$ & 7186.7(9) & $24.6(2)$ \\
\hline $\mathrm{C} 1$ & $3598.9(7)$ & $3325.4(4)$ & $4156.7(10)$ & $21.0(2)$ \\
\hline $\mathrm{C} 2$ & $3108.8(6)$ & $3613.8(4)$ & $5119.2(10)$ & $20.6(2)$ \\
\hline $\mathrm{C} 3$ & $4236.7(7)$ & $4094.8(4)$ & $5546.6(11)$ & $26.0(2)$ \\
\hline $\mathrm{C} 4$ & $4679.8(7)$ & $3825.2(4)$ & $4524.4(12)$ & $26.9(2)$ \\
\hline $\mathrm{C} 5$ & $2974.8(7)$ & $4362.0(4)$ & $6221.9(11)$ & $24.1(2)$ \\
\hline $\mathrm{C} 6$ & $3152.3(7)$ & $4878.0(4)$ & $6161.6(10)$ & $21.6(2)$ \\
\hline $\mathrm{C} 7$ & $3355.3(6)$ & $5593.3(4)$ & $5318.0(11)$ & $21.6(2)$ \\
\hline C8 & $3699.2(7)$ & $3172.4(4)$ & $1859.8(11)$ & $22.7(2)$ \\
\hline $\mathrm{C} 9$ & $3239.6(7)$ & $2716.4(4)$ & $1776.8(10)$ & $21.0(2)$ \\
\hline C10 & $2427.5(7)$ & $2720.3(4)$ & $1864.0(10)$ & $21.2(2)$ \\
\hline C11 & $2009.0(7)$ & $2302.7(4)$ & $1691.6(10)$ & $21.3(2)$ \\
\hline $\mathrm{C} 12$ & $2386.0(7)$ & $1876.7(4)$ & $1440.0(10)$ & $21.8(2)$ \\
\hline C13 & $3194.8(7)$ & $1875.7(4)$ & $1377.0(10)$ & $22.6(2)$ \\
\hline C14 & $3620.7(7)$ & $2291.0(4)$ & $1548.8(10)$ & $21.9(2)$ \\
\hline C15 & $1134.8(7)$ & $2306.6(4)$ & $1810.6(11)$ & $24.3(2)$ \\
\hline C16 & $3603.4(7)$ & $1417.8(4)$ & $1092.6(13)$ & $29.3(3)$ \\
\hline C17 & $2250.7(7)$ & $3576.7(4)$ & $4730.7(10)$ & $22.1(2)$ \\
\hline C18 & $1820.9(7)$ & $3195.8(4)$ & $5192.7(11)$ & $27.6(3)$ \\
\hline C19 & $1038.5(8)$ & $3141.6(5)$ & $4836.0(13)$ & $34.9(3)$ \\
\hline $\mathrm{C} 20$ & $676.8(8)$ & $3467.7(5)$ & $4014.1(14)$ & $37.4(3)$ \\
\hline $\mathrm{C} 21$ & 1101.6(8) & $3845.8(5)$ & $3538.3(13)$ & $35.7(3)$ \\
\hline $\mathrm{C} 22$ & 1883.8(7) & $3901.3(4)$ & $3892.4(12)$ & $28.3(3)$ \\
\hline
\end{tabular}

Table 14: Anisotropic Displacement Parameters $\left(\times 10^{4}\right)$ for yp-6-143. The anisotropic displacement factor exponent takes the form: $-2 \pi^{2}\left[h^{2} a^{* 2} \times U_{11}+\ldots+2 h k a^{*} \times b^{*} \times U_{12}\right]$

\begin{tabular}{lrrrrrr}
\hline Atom & $\boldsymbol{U}_{\mathbf{1 1}}$ & $\boldsymbol{U}_{\mathbf{2 2}}$ & $\boldsymbol{U}_{\mathbf{3 3}}$ & $\boldsymbol{U}_{\mathbf{2 3}}$ & $\boldsymbol{U}_{\mathbf{1 3}}$ & $\boldsymbol{U}_{\mathbf{1 2}}$ \\
\hline F1 & $32.8(4)$ & $47.8(5)$ & $26.3(4)$ & $7.9(3)$ & $8.0(3)$ & $2.5(3)$ \\
F2 & $29.5(4)$ & $40.5(4)$ & $45.2(5)$ & $14.2(3)$ & $2.0(3)$ & $8.5(3)$ \\
F3 & $28.1(4)$ & $57.5(5)$ & $58.9(5)$ & $-35.0(4)$ & $0.5(4)$ & $-8.2(4)$ \\
F4 & $101.7(8)$ & $42.5(5)$ & $37.7(5)$ & $-15.6(4)$ & $4.5(5)$ & $25.9(5)$ \\
F5 & $32.1(4)$ & $33.1(4)$ & $100.4(8)$ & $-8.2(5)$ & $5.2(5)$ & $8.0(3)$ \\
F6 & $56.1(5)$ & $23.0(4)$ & $70.9(6)$ & $14.3(4)$ & $25.6(4)$ & $8.0(3)$ \\
O1 & $28.0(4)$ & $22.2(4)$ & $26.5(4)$ & $-1.1(3)$ & $-3.3(3)$ & $2.7(3)$ \\
O2 & $29.5(4)$ & $17.1(4)$ & $15.4(4)$ & $0.0(3)$ & $-0.1(3)$ & $0.1(3)$ \\
O3 & $37.8(5)$ & $19.9(4)$ & $22.9(4)$ & $3.8(3)$ & $-2.5(3)$ & $-0.8(3)$ \\
N1 & $27.0(5)$ & $16.6(4)$ & $17.6(4)$ & $-1.6(3)$ & $0.7(3)$ & $-2.3(3)$ \\
N2 & $32.7(5)$ & $18.6(5)$ & $15.0(4)$ & $-1.4(3)$ & $-0.4(4)$ & $-1.5(4)$ \\
N3 & $40.8(6)$ & $15.4(5)$ & $19.1(5)$ & $-1.9(4)$ & $-0.7(4)$ & $-1.2(4)$
\end{tabular}




\begin{tabular}{lrrrrrr}
\hline Atom & $\boldsymbol{U}_{11}$ & $\boldsymbol{U}_{22}$ & $\boldsymbol{U}_{33}$ & $\boldsymbol{U}_{23}$ & $\boldsymbol{U}_{13}$ & $\boldsymbol{U}_{12}$ \\
\hline N4 & $38.0(5)$ & $17.7(4)$ & $18.1(4)$ & $-0.6(3)$ & $1.2(4)$ & $-1.4(4)$ \\
C1 & $29.1(6)$ & $16.6(5)$ & $17.2(5)$ & $1.6(4)$ & $-1.7(4)$ & $-0.5(4)$ \\
C2 & $29.6(6)$ & $16.7(5)$ & $15.5(5)$ & $1.2(4)$ & $-0.2(4)$ & $-2.4(4)$ \\
C3 & $30.1(6)$ & $22.9(6)$ & $24.8(6)$ & $-2.4(4)$ & $-4.5(4)$ & $-3.6(4)$ \\
C4 & $25.5(6)$ & $25.1(6)$ & $29.9(6)$ & $-1.2(5)$ & $-2.6(5)$ & $-2.0(4)$ \\
C5 & $35.2(6)$ & $19.3(5)$ & $17.8(5)$ & $-1.3(4)$ & $3.8(4)$ & $-3.4(4)$ \\
C6 & $28.3(5)$ & $19.7(5)$ & $17.0(5)$ & $-0.5(4)$ & $1.2(4)$ & $-0.6(4)$ \\
C7 & $25.9(5)$ & $18.9(5)$ & $19.9(5)$ & $-0.4(4)$ & $-1.8(4)$ & $0.8(4)$ \\
C8 & $28.9(6)$ & $20.7(5)$ & $18.7(5)$ & $-2.5(4)$ & $3.8(4)$ & $-2.5(4)$ \\
C9 & $28.6(6)$ & $21.0(5)$ & $13.4(5)$ & $-0.4(4)$ & $0.2(4)$ & $-2.0(4)$ \\
C10 & $29.3(6)$ & $19.0(5)$ & $15.3(5)$ & $-0.8(4)$ & $0.1(4)$ & $1.6(4)$ \\
C11 & $26.7(6)$ & $23.2(5)$ & $14.0(5)$ & $0.0(4)$ & $-1.0(4)$ & $-0.5(4)$ \\
C12 & $29.9(6)$ & $19.5(5)$ & $16.0(5)$ & $-1.0(4)$ & $0.0(4)$ & $-3.1(4)$ \\
C13 & $30.4(6)$ & $20.5(5)$ & $17.0(5)$ & $0.1(4)$ & $2.2(4)$ & $0.6(4)$ \\
C14 & $24.9(5)$ & $23.5(6)$ & $17.2(5)$ & $0.3(4)$ & $1.6(4)$ & $-0.3(4)$ \\
C15 & $28.6(6)$ & $24.3(6)$ & $20.1(5)$ & $-3.2(4)$ & $0.0(4)$ & $-1.2(4)$ \\
C16 & $34.1(6)$ & $21.6(6)$ & $32.3(6)$ & $0.0(5)$ & $7.1(5)$ & $0.7(5)$ \\
C17 & $29.0(6)$ & $21.1(5)$ & $16.3(5)$ & $-4.1(4)$ & $2.9(4)$ & $-2.1(4)$ \\
C18 & $36.1(6)$ & $26.5(6)$ & $20.2(5)$ & $-2.6(4)$ & $3.0(5)$ & $-6.6(5)$ \\
C19 & $37.4(7)$ & $39.2(7)$ & $28.3(6)$ & $-6.3(5)$ & $5.5(5)$ & $-15.0(6)$ \\
C20 & $27.6(6)$ & $51.9(8)$ & $32.7(7)$ & $-9.5(6)$ & $0.7(5)$ & $-6.1(6)$ \\
C21 & $33.2(7)$ & $42.3(7)$ & $31.5(6)$ & $-0.1(5)$ & $-3.3(5)$ & $4.4(6)$ \\
C22 & $31.6(6)$ & $27.6(6)$ & $25.7(6)$ & $1.4(5)$ & $0.8(5)$ & $-0.7(5)$
\end{tabular}

Table 15: Bond Lengths in Å for yp-6-143.

\begin{tabular}{lll}
\hline Atom & Atom & Length/Å \\
\hline F1 & C15 & $1.3335(13)$ \\
F2 & C15 & $1.3351(14)$ \\
F3 & C15 & $1.3286(13)$ \\
F4 & C16 & $1.3315(16)$ \\
F5 & C16 & $1.3246(16)$ \\
F6 & C16 & $1.3259(14)$ \\
O1 & C1 & $1.3985(14)$ \\
O1 & C4 & $1.4363(14)$ \\
O2 & C1 & $1.4209(12)$ \\
O2 & C8 & $1.4351(13)$ \\
O3 & C7 & $1.2299(14)$ \\
N1 & C2 & $1.4728(13)$ \\
N1 & C3 & $1.4734(14)$ \\
N1 & C5 & $1.4707(14)$ \\
N2 & C6 & $1.3642(14)$ \\
N2 & C7 & $1.3797(14)$ \\
N3 & N4 & $1.3881(13)$ \\
N3 & C7 & $1.3586(14)$ \\
N4 & C6 & $1.3002(14)$
\end{tabular}

\begin{tabular}{lll}
\hline Atom & Atom & Length/̊̊ \\
\hline C1 & C2 & $1.5362(15)$ \\
C2 & C17 & $1.5156(15)$ \\
C3 & C4 & $1.5062(16)$ \\
C5 & C6 & $1.4907(15)$ \\
C8 & C9 & $1.5109(15)$ \\
C9 & C10 & $1.3949(16)$ \\
C9 & C14 & $1.3895(15)$ \\
C10 & C11 & $1.3893(15)$ \\
C11 & C12 & $1.3922(15)$ \\
C11 & C15 & $1.5041(16)$ \\
C12 & C13 & $1.3874(16)$ \\
C13 & C14 & $1.3902(16)$ \\
C13 & C16 & $1.5020(16)$ \\
C17 & C18 & $1.3914(16)$ \\
C17 & C22 & $1.3912(16)$ \\
C18 & C19 & $1.3877(18)$ \\
C19 & C20 & $1.380(2)$ \\
C20 & C21 & $1.385(2)$ \\
C21 & C22 & $1.3872(18)$
\end{tabular}

Table 16: Bond Angles in ${ }^{\circ}$ for yp-6-143.

\begin{tabular}{lllc}
\hline Atom & Atom & Atom & Angle $^{\circ}$ \\
\hline C1 & O1 & C4 & $112.02(8)$ \\
C1 & O2 & C8 & $113.00(8)$ \\
C2 & N1 & C3 & $108.60(8)$ \\
C5 & N1 & C2 & $109.62(8)$
\end{tabular}

\begin{tabular}{lllc}
\hline Atom & Atom & Atom & Angle $^{\circ}$ \\
\hline C5 & N1 & C3 & $109.40(8)$ \\
C6 & N2 & C7 & $108.38(9)$ \\
C7 & N3 & N4 & $112.65(9)$ \\
C6 & N4 & N3 & $103.97(9)$
\end{tabular}




\begin{tabular}{llll}
\hline Atom & Atom & Atom & Angle $^{\circ}$ \\
\hline O1 & C1 & O2 & $111.19(9)$ \\
O1 & C1 & C2 & $113.69(9)$ \\
O2 & C1 & C2 & $108.16(8)$ \\
N1 & C2 & C1 & $110.33(9)$ \\
N1 & C2 & C17 & $113.58(9)$ \\
C17 & C2 & C1 & $109.75(8)$ \\
N1 & C3 & C4 & $110.40(9)$ \\
O1 & C4 & C3 & $110.66(9)$ \\
N1 & C5 & C6 & $110.56(9)$ \\
N2 & C6 & C5 & $123.72(10)$ \\
N4 & C6 & N2 & $111.86(10)$ \\
N4 & C6 & C5 & $124.37(10)$ \\
O3 & C7 & N2 & $128.21(10)$ \\
O3 & C7 & N3 & $128.64(10)$ \\
N3 & C7 & N2 & $103.13(9)$ \\
O2 & C8 & C9 & $112.43(9)$ \\
C10 & C9 & C8 & $120.53(10)$ \\
C14 & C9 & C8 & $120.07(10)$ \\
C14 & C9 & C10 & $119.32(10)$ \\
C11 & C10 & C9 & $119.80(10)$ \\
C10 & C11 & C12 & $121.17(10)$ \\
C10 & C11 & C15 & $119.65(10)$ \\
C12 & C11 & C15 & $119.16(10)$ \\
C13 & C12 & C11 & $118.51(10)$ \\
C12 & C13 & C14 & $120.89(10)$ \\
& & &
\end{tabular}

\begin{tabular}{llll}
\hline Atom & Atom & Atom & \multicolumn{1}{c}{ Angle $^{\circ}$} \\
\hline C12 & C13 & C16 & $118.69(10)$ \\
C14 & C13 & C16 & $120.41(10)$ \\
C9 & C14 & C13 & $120.28(10)$ \\
F1 & C15 & F2 & $106.17(9)$ \\
F1 & C15 & C11 & $112.07(9)$ \\
F2 & C15 & C11 & $112.35(9)$ \\
F3 & C15 & F1 & $106.97(10)$ \\
F3 & C15 & F2 & $106.72(10)$ \\
F3 & C15 & C11 & $112.15(9)$ \\
F4 & C16 & C13 & $111.72(10)$ \\
F5 & C16 & F4 & $106.20(11)$ \\
F5 & C16 & F6 & $107.24(11)$ \\
F5 & C16 & C13 & $112.75(10)$ \\
F6 & C16 & F4 & $106.13(11)$ \\
F6 & C16 & C13 & $112.35(10)$ \\
C18 & C17 & C2 & $118.85(10)$ \\
C18 & C17 & C22 & $118.77(11)$ \\
C22 & C17 & C2 & $122.34(10)$ \\
C19 & C18 & C17 & $120.80(12)$ \\
C20 & C19 & C18 & $120.06(12)$ \\
C19 & C20 & C21 & $119.60(12)$ \\
C20 & C21 & C22 & $120.53(12)$ \\
C21 & C22 & C17 & $120.24(12)$
\end{tabular}

Table 17: Torsion Angles in ${ }^{\circ}$ for yp-6-143.

\begin{tabular}{llllc}
\hline Atom & Atom & Atom & Atom & Angle $/^{\circ}$ \\
\hline O1 & C1 & C2 & N1 & $53.05(11)$ \\
O1 & C1 & C2 & C17 & $178.94(8)$ \\
O2 & C1 & C2 & N1 & $-70.97(11)$ \\
O2 & C1 & C2 & C17 & $54.92(11)$ \\
O2 & C8 & C9 & C10 & $41.74(14)$ \\
O2 & C8 & C9 & C14 & $-141.36(10$ \\
& & & & ) \\
N1 & C2 & C17 & C18 & $-150.03(10$ \\
& & & & ) \\
N1 & C2 & C17 & C22 & $32.20(14)$ \\
N1 & C3 & C4 & O1 & $-59.83(12)$ \\
N1 & C5 & C6 & N2 & $39.01(15)$ \\
N1 & C5 & C6 & N4 & $-143.75(11$ \\
& & & & ) \\
N3 & N4 & C6 & N2 & $0.14(13)$ \\
N3 & N4 & C6 & C5 & $-177.39(11$ \\
& & & & ) \\
N4 & N3 & C7 & 03 & $-178.87(11$ \\
& & & & ) \\
N4 & N3 & C7 & N2 & $-0.26(13)$ \\
C1 & O1 & C4 & C3 & $56.07(12)$ \\
C1 & O2 & C8 & C9 & $71.80(11)$ \\
C1 & C2 & C17 & C18 & $85.95(12)$ \\
C1 & C2 & C17 & C22 & $-91.82(12)$ \\
C2 & N1 & C3 & C4 & $59.40(11)$ \\
C2 & N1 & C5 & C6 & $-170.01(9)$ \\
C2 & C17 & C18 & C19 & $-178.44(10$
\end{tabular}




\begin{tabular}{|c|c|c|c|c|}
\hline Atom & Atom & Atom & Atom & Angle $/^{\circ}$ \\
\hline $\mathrm{C} 2$ & C17 & $\mathrm{C} 22$ & $\mathrm{C} 21$ & 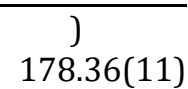 \\
\hline $\mathrm{C} 3$ & N1 & $\mathrm{C} 2$ & $\mathrm{C} 1$ & $-54.77(11)$ \\
\hline C3 & N1 & $\mathrm{C} 2$ & C17 & $-178.48(8)$ \\
\hline C3 & N1 & C5 & C6 & $71.01(11)$ \\
\hline $\mathrm{C} 4$ & 01 & $\mathrm{C} 1$ & 02 & $69.23(11)$ \\
\hline $\mathrm{C} 4$ & 01 & C1 & $\mathrm{C} 2$ & $-53.13(11)$ \\
\hline C5 & N1 & $\mathrm{C} 2$ & $\mathrm{C} 1$ & $-174.24(9)$ \\
\hline C5 & N1 & $\mathrm{C} 2$ & C17 & $62.06(11)$ \\
\hline C5 & N1 & C3 & C4 & $179.00(9)$ \\
\hline C6 & $\mathrm{N} 2$ & C7 & 03 & $178.94(11)$ \\
\hline C6 & $\mathrm{N} 2$ & C7 & N3 & $0.33(12)$ \\
\hline C7 & $\mathrm{N} 2$ & C6 & N4 & $-0.31(13)$ \\
\hline $\mathrm{C} 7$ & N2 & C6 & $\mathrm{C} 5$ & $177.24(10)$ \\
\hline C7 & N3 & N4 & $\mathrm{C} 6$ & $0.08(13)$ \\
\hline $\mathrm{C} 8$ & 02 & C1 & 01 & $68.07(11)$ \\
\hline C8 & 02 & $\mathrm{C} 1$ & $\mathrm{C} 2$ & $-166.43(8)$ \\
\hline $\mathrm{C} 8$ & C9 & C10 & C11 & $175.40(9)$ \\
\hline $\mathrm{C} 8$ & C9 & C14 & C13 & $\begin{array}{c}-175.35(10 \\
)\end{array}$ \\
\hline C9 & C10 & C11 & C12 & $0.41(16)$ \\
\hline C9 & C10 & C11 & C15 & $178.90(9)$ \\
\hline C10 & C9 & C14 & $\mathrm{C} 13$ & $1.58(16)$ \\
\hline C10 & C11 & C12 & $\mathrm{C} 13$ & $0.64(16)$ \\
\hline $\mathrm{C} 10$ & C11 & C15 & $\mathrm{F} 1$ & $-88.82(12)$ \\
\hline C10 & C11 & C15 & F2 & $30.63(14)$ \\
\hline C10 & C11 & C15 & F3 & $150.84(10)$ \\
\hline C11 & C12 & $\mathrm{C} 13$ & C14 & $-0.58(16)$ \\
\hline C11 & $\mathrm{C} 12$ & $\mathrm{C} 13$ & C16 & $\begin{array}{c}-179.41(10 \\
)\end{array}$ \\
\hline C12 & C11 & C15 & F1 & $89.70(12)$ \\
\hline C12 & C11 & C15 & F2 & $\begin{array}{c}-150.85(10 \\
)\end{array}$ \\
\hline C12 & C11 & C15 & F3 & $-30.64(14)$ \\
\hline C12 & C13 & C14 & C9 & $-0.53(16)$ \\
\hline C12 & C13 & C16 & F4 & $74.58(14)$ \\
\hline C12 & $\mathrm{C} 13$ & C16 & F5 & $\begin{array}{c}-165.89(10 \\
)\end{array}$ \\
\hline C12 & C13 & C16 & F6 & $-44.57(15)$ \\
\hline C14 & C9 & C10 & C11 & $-1.52(15)$ \\
\hline C14 & C13 & C16 & F4 & $\begin{array}{c}-104.25(13 \\
)\end{array}$ \\
\hline C14 & C13 & C16 & F5 & $15.28(16)$ \\
\hline C14 & C13 & C16 & F6 & $136.59(11)$ \\
\hline C15 & C11 & C12 & C13 & $-177.86(9)$ \\
\hline C16 & C13 & C14 & C9 & $178.28(10)$ \\
\hline C17 & C18 & C19 & $\mathrm{C} 20$ & $-0.03(19)$ \\
\hline C18 & C17 & $\mathrm{C} 22$ & $\mathrm{C} 21$ & $0.58(17)$ \\
\hline C18 & C19 & $\mathrm{C} 20$ & $\mathrm{C} 21$ & $0.7(2)$ \\
\hline C19 & $\mathrm{C} 20$ & $\mathrm{C} 21$ & $\mathrm{C} 22$ & $-0.7(2)$ \\
\hline $\mathrm{C} 20$ & $\mathrm{C} 21$ & $\mathrm{C} 22$ & C17 & $0.03(19)$ \\
\hline $\mathrm{C} 22$ & C17 & C18 & C19 & $-0.59(17)$ \\
\hline
\end{tabular}

Table 18: Hydrogen Fractional Atomic Coordinates $\left(\times 10^{4}\right)$ and Equivalent Isotropic Displacement Parameters $\left(\AA^{2} \times 10^{3}\right)$ for $\mathbf{y p - 6 - 1 4 3}$. $U_{e q}$ is defined as $1 / 3$ of the trace of the orthogonalised $U_{i j}$. 


\begin{tabular}{lclll}
\hline Atom & \multicolumn{1}{c}{$\mathbf{x}$} & $\mathbf{y}$ & $\mathbf{z}$ & \multicolumn{1}{c}{$\boldsymbol{U}_{\text {eq }}$} \\
\hline H2 & $3210(9)$ & $5012(6)$ & $4238(17)$ & $33(4)$ \\
H3 & $3381(9)$ & $5854(6)$ & $7150(16)$ & $35(4)$ \\
H1 & 3433.29 & 2986.94 & 4219.76 & 25 \\
H2A & 3178.98 & 3468.62 & 6012.51 & 25 \\
H3A & 4315.9 & 3942.65 & 6417.86 & 31 \\
H3B & 4438.65 & 4422.65 & 5606.6 & 31 \\
H4A & 4613.85 & 3983.39 & 3658.85 & 32 \\
H4B & 5243.62 & 3824.75 & 4765.1 & 32 \\
H5A & 3130.07 & 4237.31 & 7100.87 & 29 \\
H5B & 2405.62 & 4312.26 & 6094.05 & 29 \\
H8A & 4255.41 & 3098.17 & 2052.25 & 27 \\
H8B & 3667.08 & 3334.78 & 996.07 & 27 \\
H10 & 2161.42 & 3007.68 & 2040.75 & 25 \\
H12 & 2095.93 & 1592.98 & 1314.21 & 26 \\
H14 & 4174.56 & 2283.94 & 1510.12 & 26 \\
H18 & 2065.92 & 2969.98 & 5759.22 & 33 \\
H19 & 751.53 & 2879.78 & 5157.85 & 42 \\
H20 & 139.87 & 3433.13 & 3776.06 & 45 \\
H21 & 855.18 & 4068.89 & 2965 & 43 \\
H22 & 2169.85 & 4161.9 & 3561.15 & 34
\end{tabular}


Table 19: Solvent masking (PLATON/SQUEEZE) information for yp-6-143.

\begin{tabular}{lllrrrl}
\hline No & $\mathbf{x}$ & $\mathbf{y}$ & $\mathbf{z}$ & $\mathbf{V}$ & \multicolumn{1}{c}{$\mathbf{e}$} & Content \\
\hline 1 & 0.000 & 0.000 & -0.553 & 85.1 & 0.3 & $?$ \\
2 & 0.000 & 0.500 & -0.604 & 351.6 & 59.5 & 1.2 hexane \\
3 & 0.500 & 0.000 & -0.715 & 351.6 & 59.5 & 1.2 hexane \\
4 & 0.500 & 0.500 & -0.928 & 85.1 & 0.3 & $?$
\end{tabular}




\section{Copies of NMR spectra and SFC chromatograms}

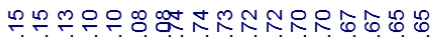

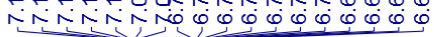

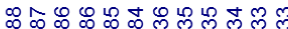

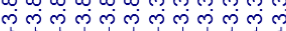

3b, ${ }^{1} \mathrm{H}$ NMR (400 MHz, $\mathrm{CDCl}_{3}$ )
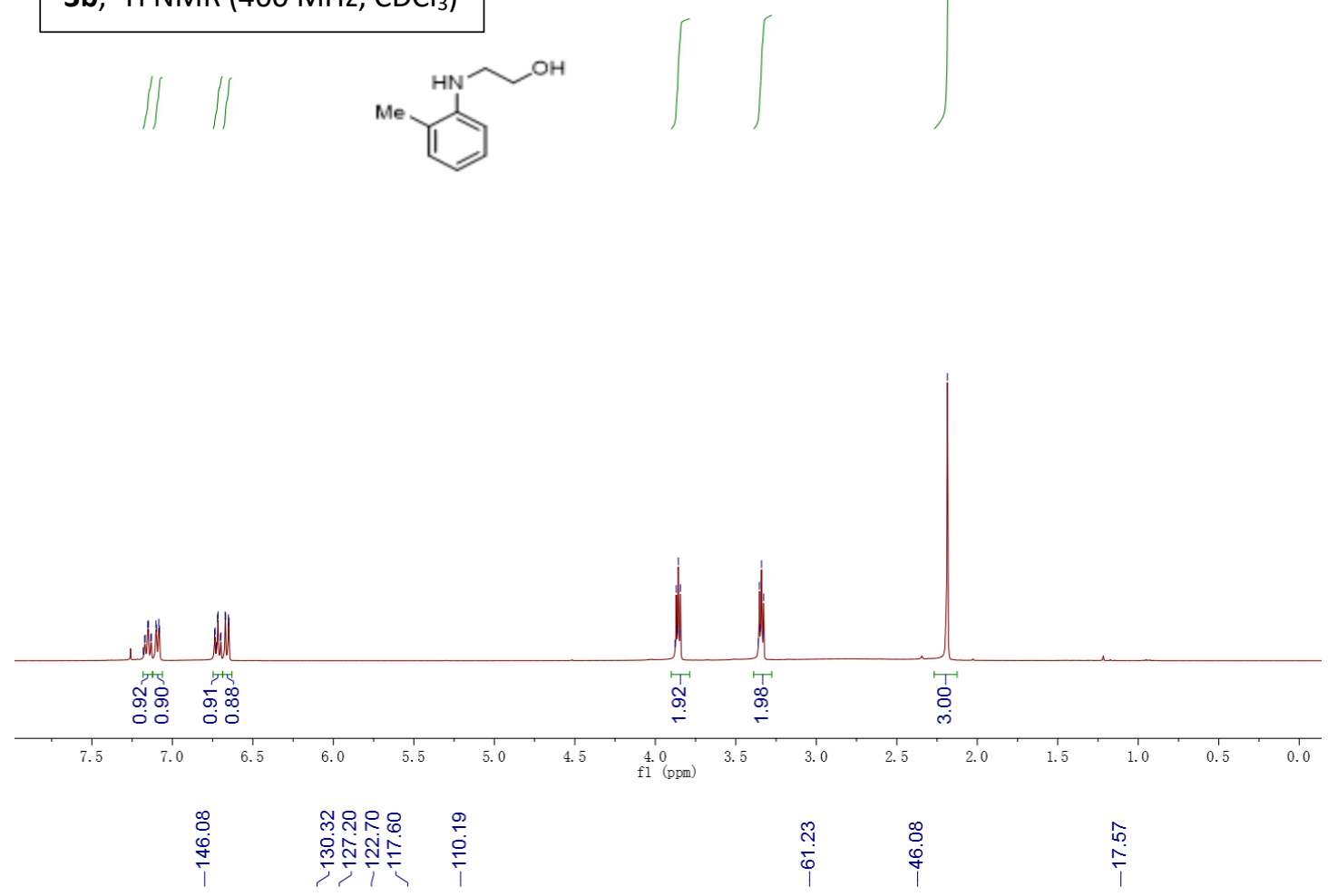

3b, ${ }^{13} \mathrm{C}$ NMR (101 MHz, $\mathrm{CDCl}_{3}$ )
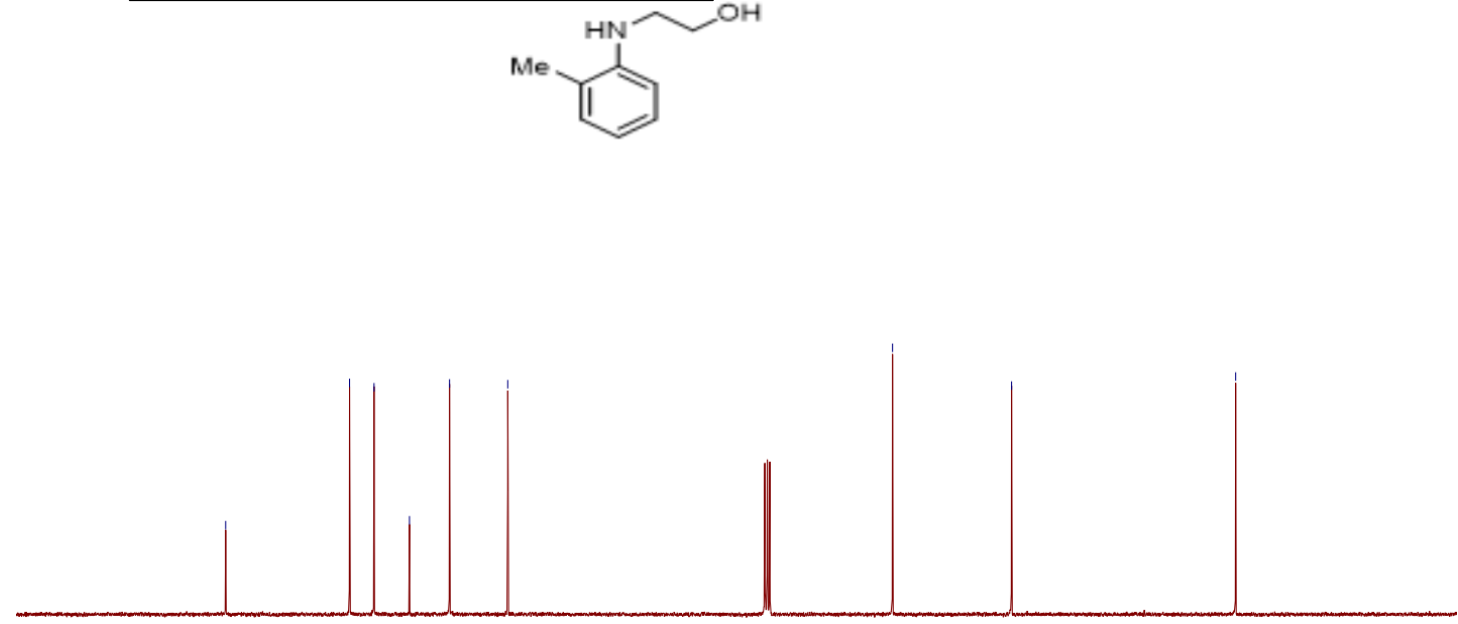

$\frac{1}{170} \quad 160$

$150 \quad 140$

$130 \quad 120 \quad 110 \quad 100$

f1 $\stackrel{80}{(\mathrm{ppm})}$ 


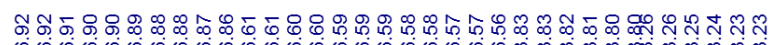

प0
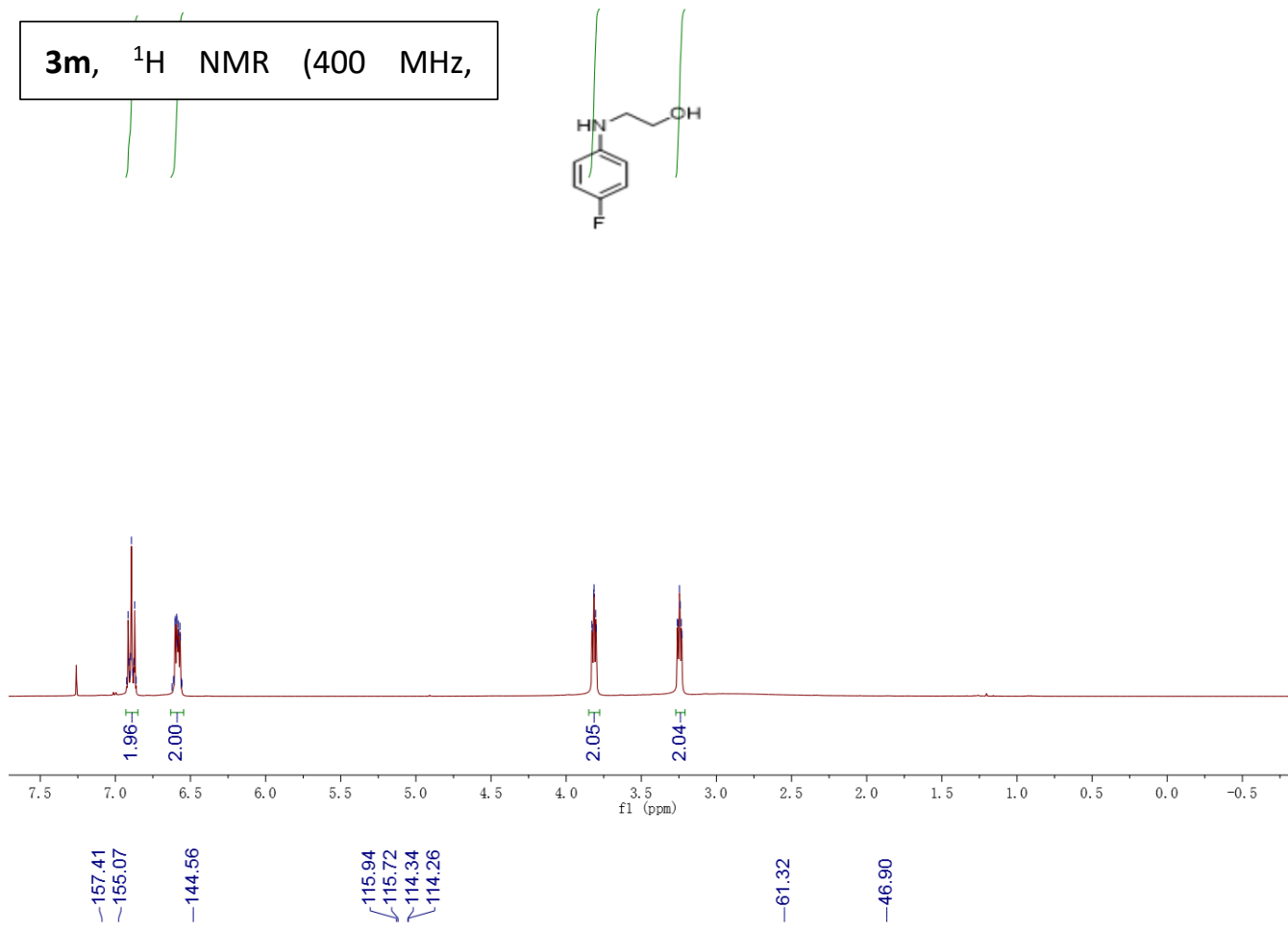

3m, ${ }^{13} \mathrm{C}$ NMR (101 MHz, $\mathrm{CDCl}_{3}$ )<smiles>OCCNc1ccc(F)cc1</smiles>
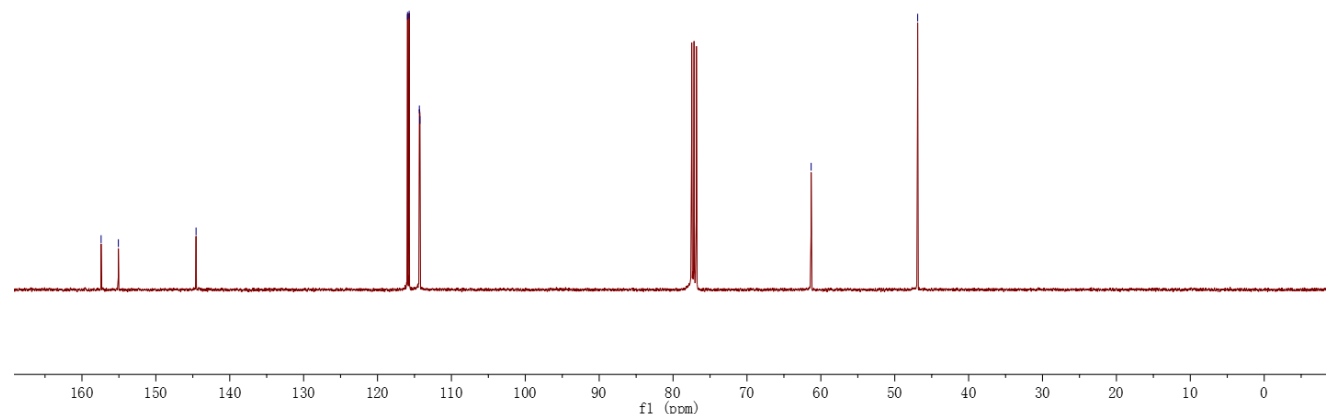


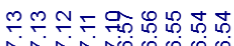

ivinino

mim

3p, ${ }^{1} \mathrm{H}$ NMR (400 MHz, $\left.\mathrm{CDCl}_{3}\right)$
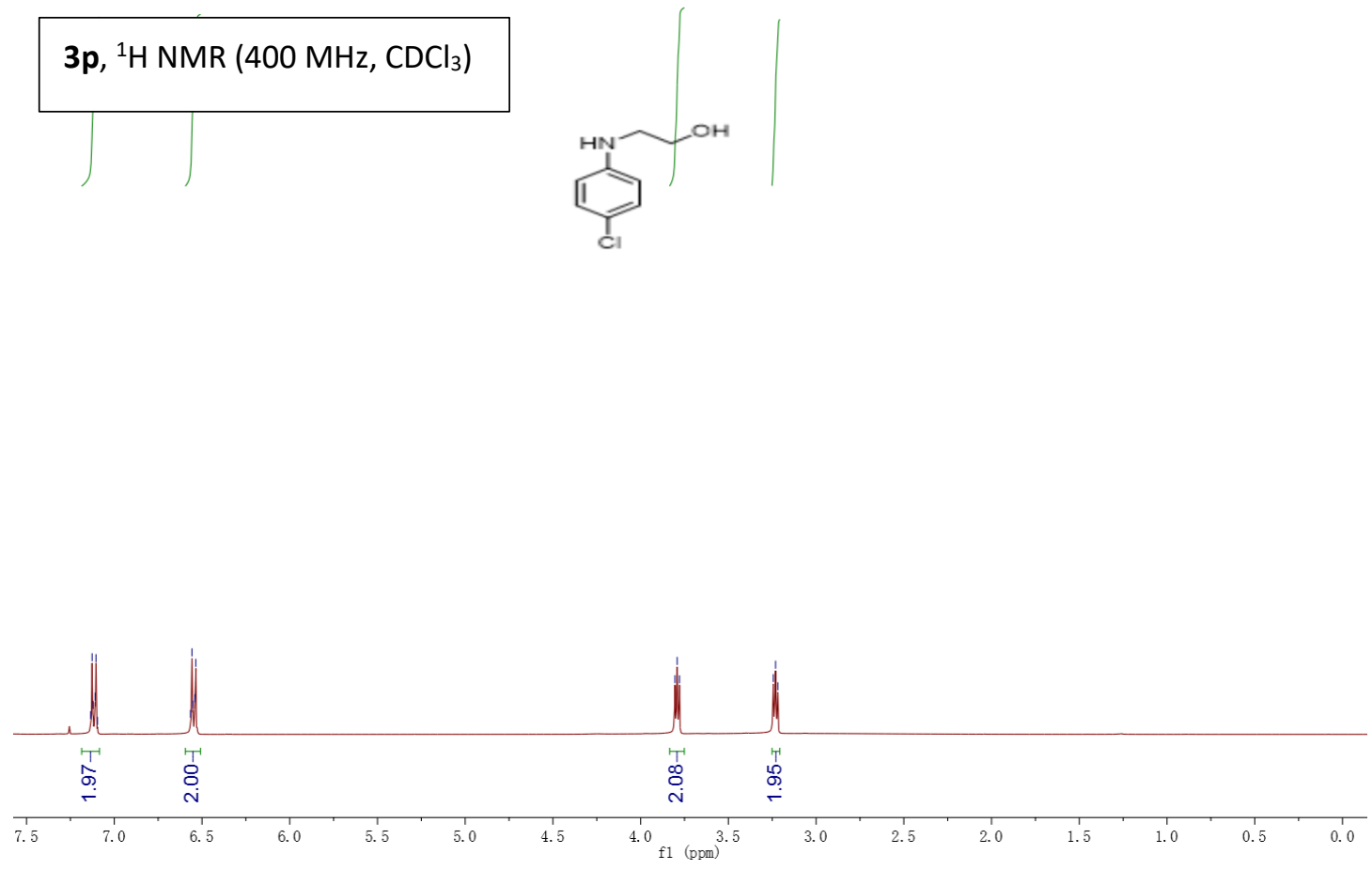

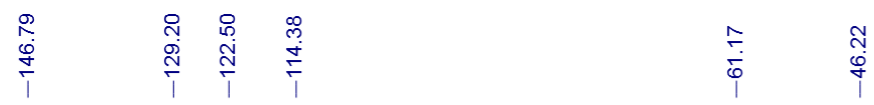

3p, ${ }^{13} \mathrm{C}$ NMR $\left(101 \mathrm{MHz}, \mathrm{CDCl}_{3}\right)$<smiles>OCCNc1ccc(Cl)cc1</smiles>

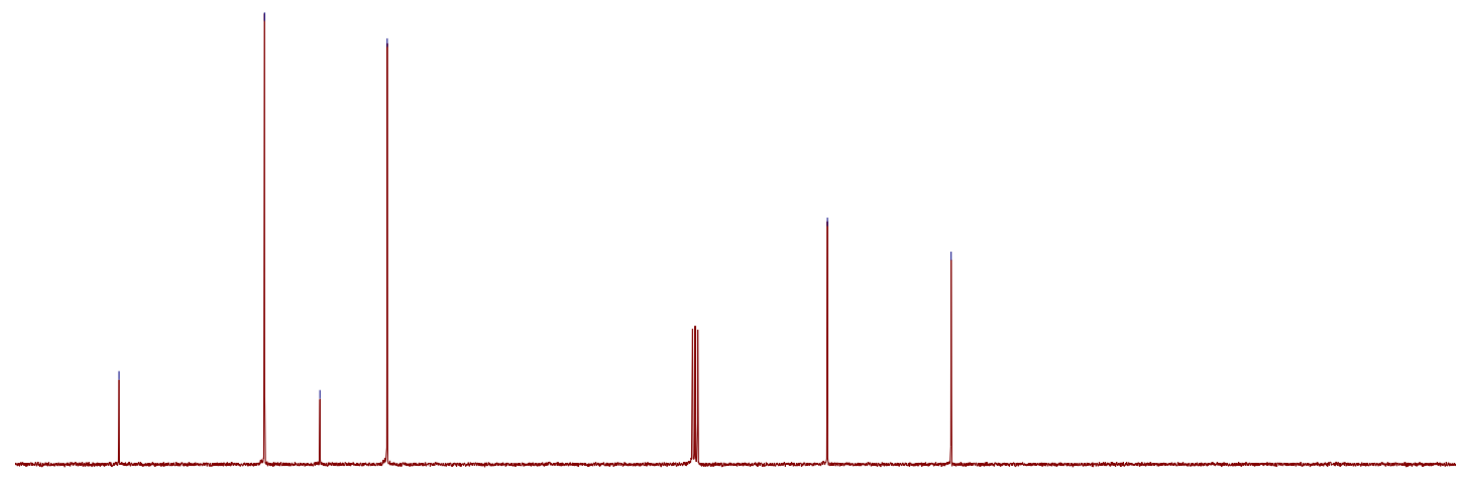

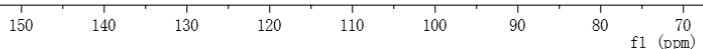



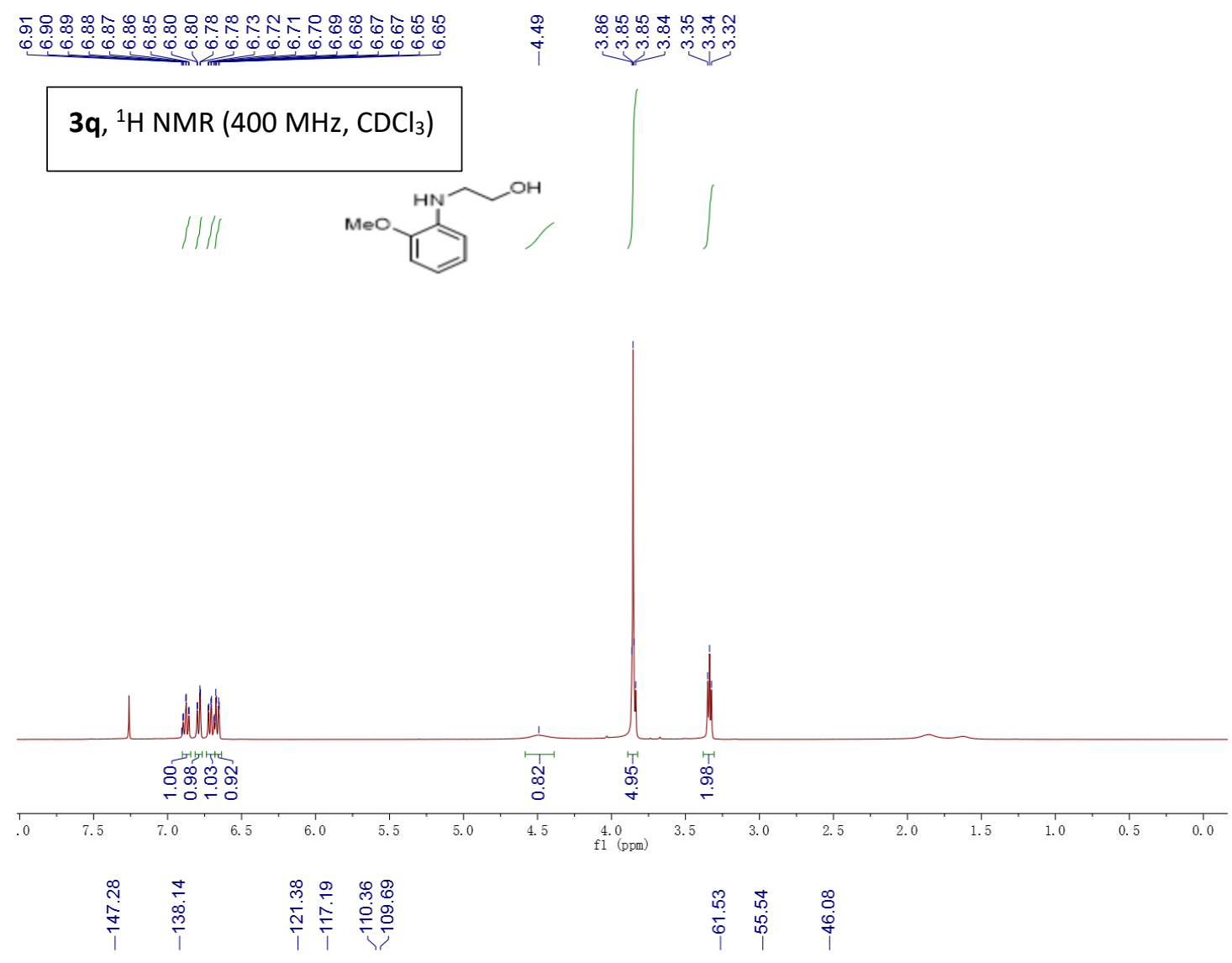

$$
\text { 3q, } \left.{ }^{13} \mathrm{C} \mathrm{NMR} \mathrm{(101} \mathrm{MHz,} \mathrm{CDCl}_{3}\right)
$$
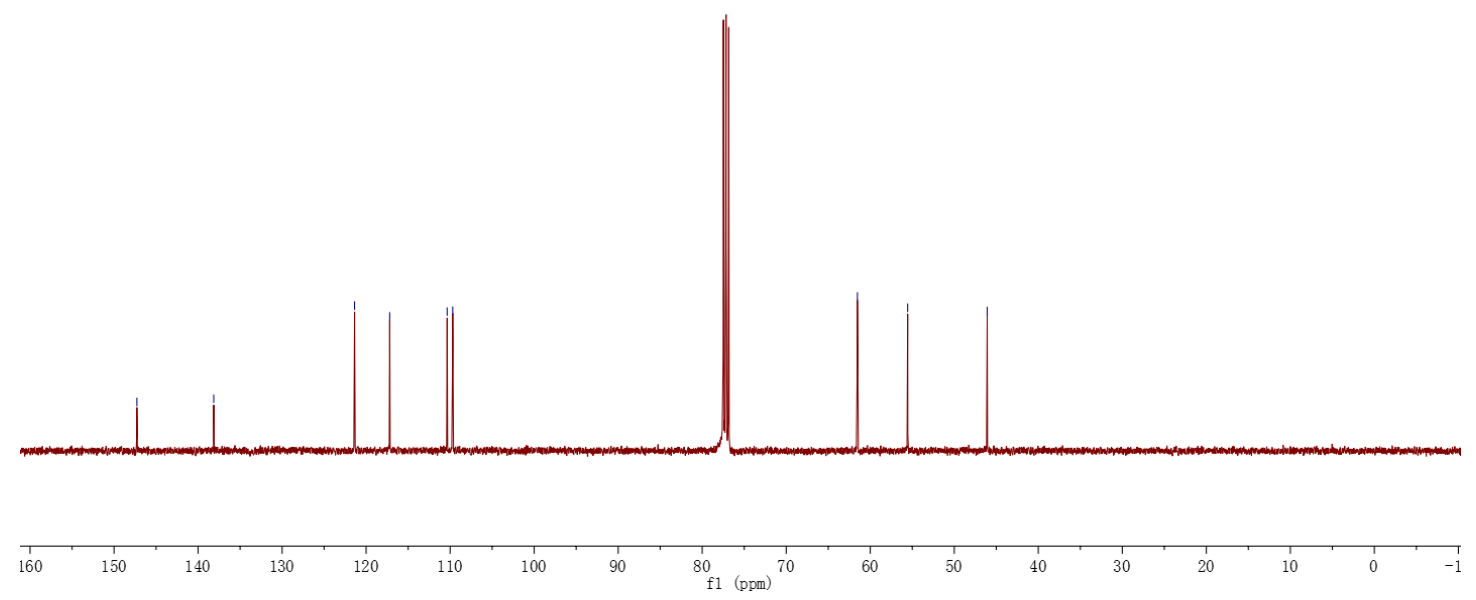

S63 


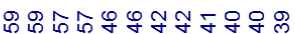

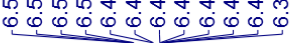

3s, ${ }^{1} \mathrm{H}$ NMR (400 MHz, $\mathrm{CDCl}_{3}$ )

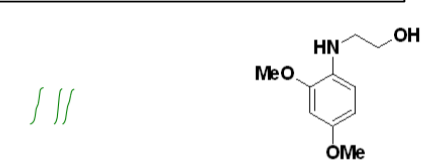

OMe
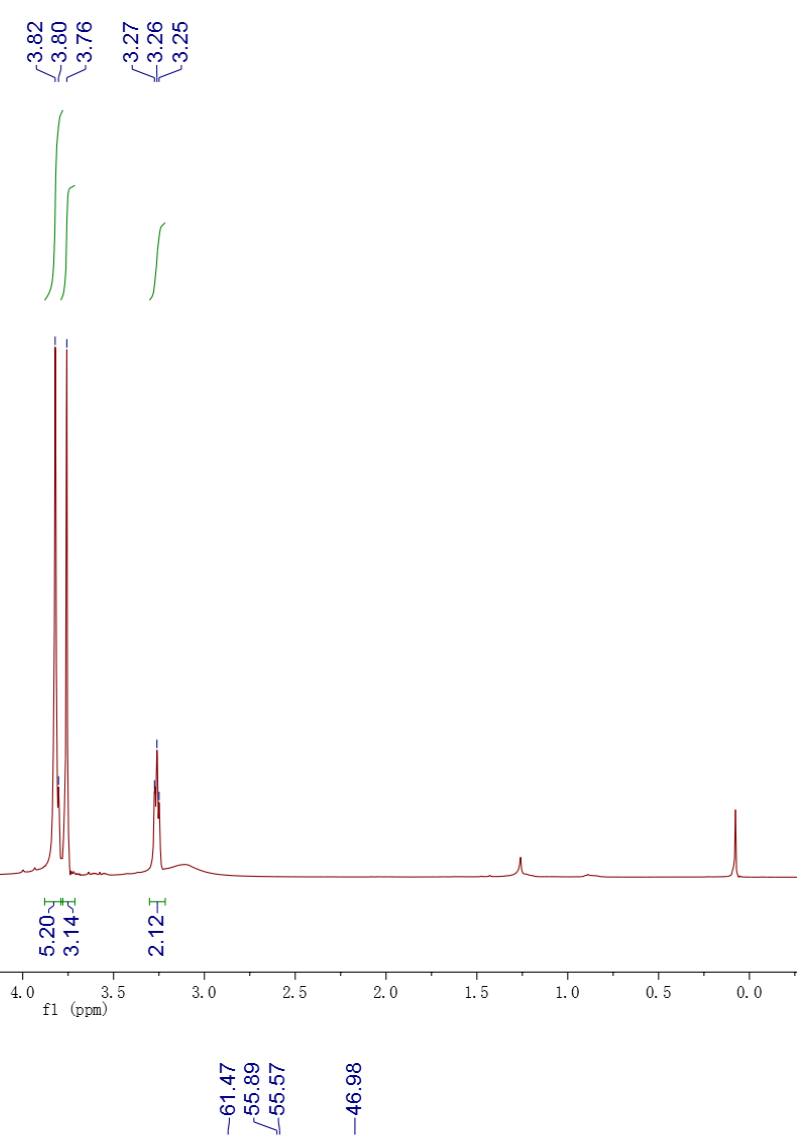

3s, ${ }^{13} \mathrm{C}$ NMR (101 MHz, CDCl $)$
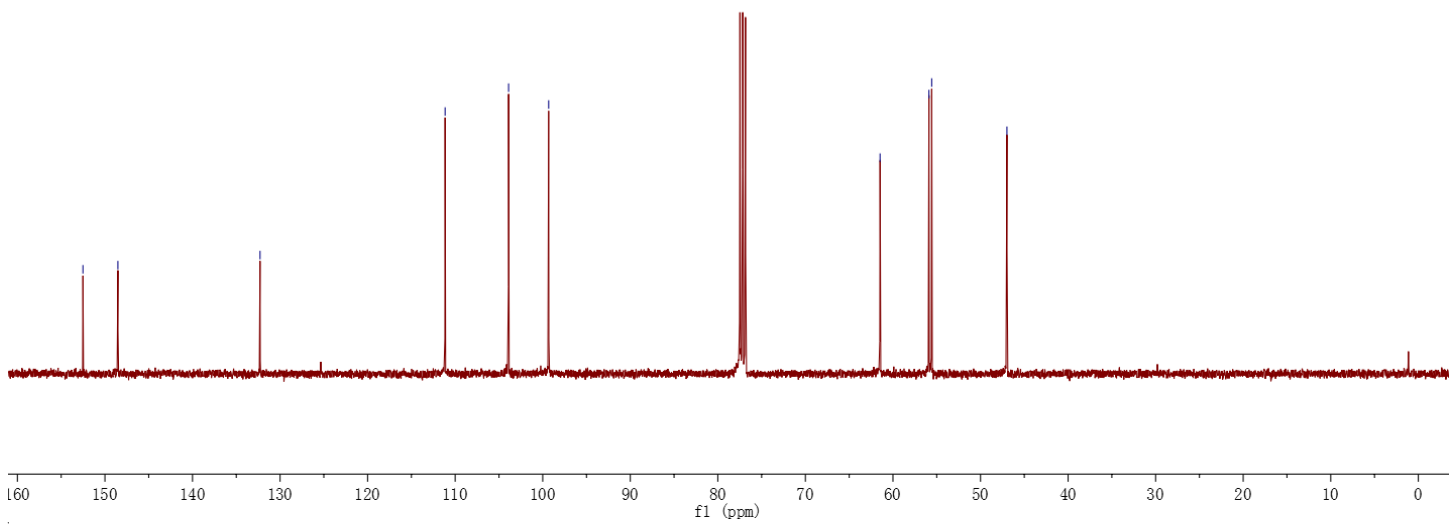

S64 


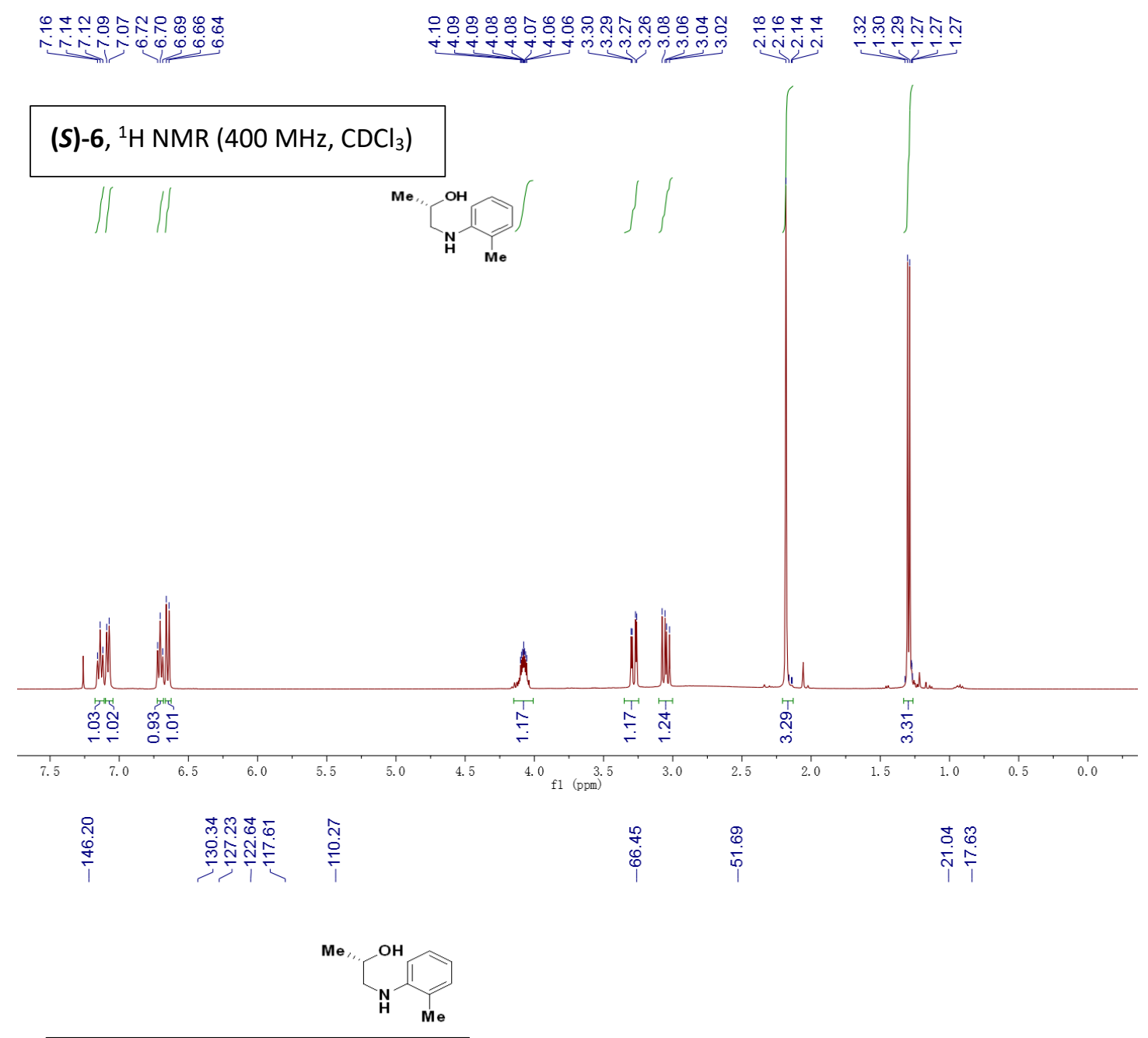

(S)-6, ${ }^{13} \mathrm{C}$ NMR (101 MHz, CDCl 3 )
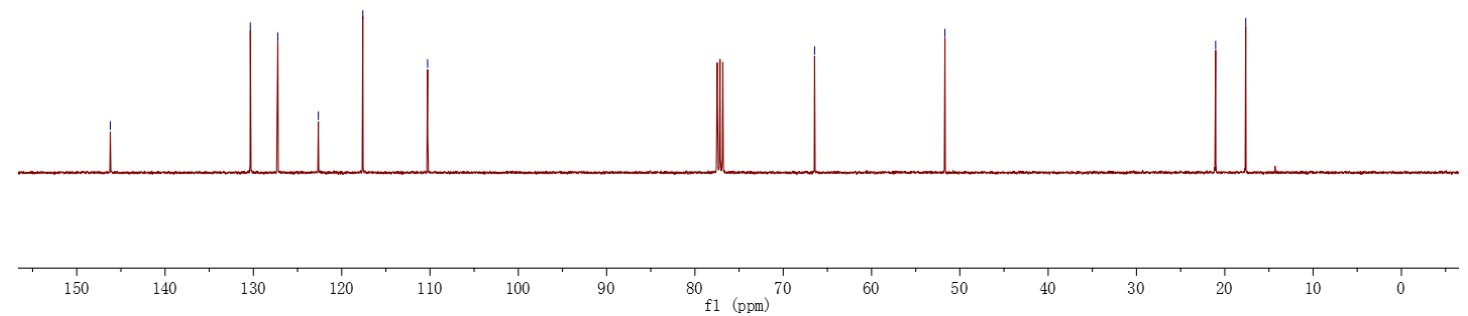


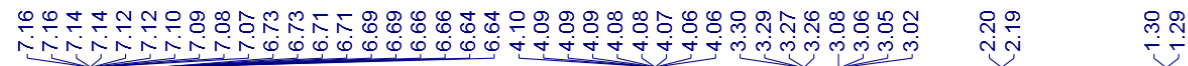

(R)-6, ${ }^{1} \mathrm{H}$ NMR $\left(400 \mathrm{MHz}, \mathrm{CDCl}_{3}\right)$

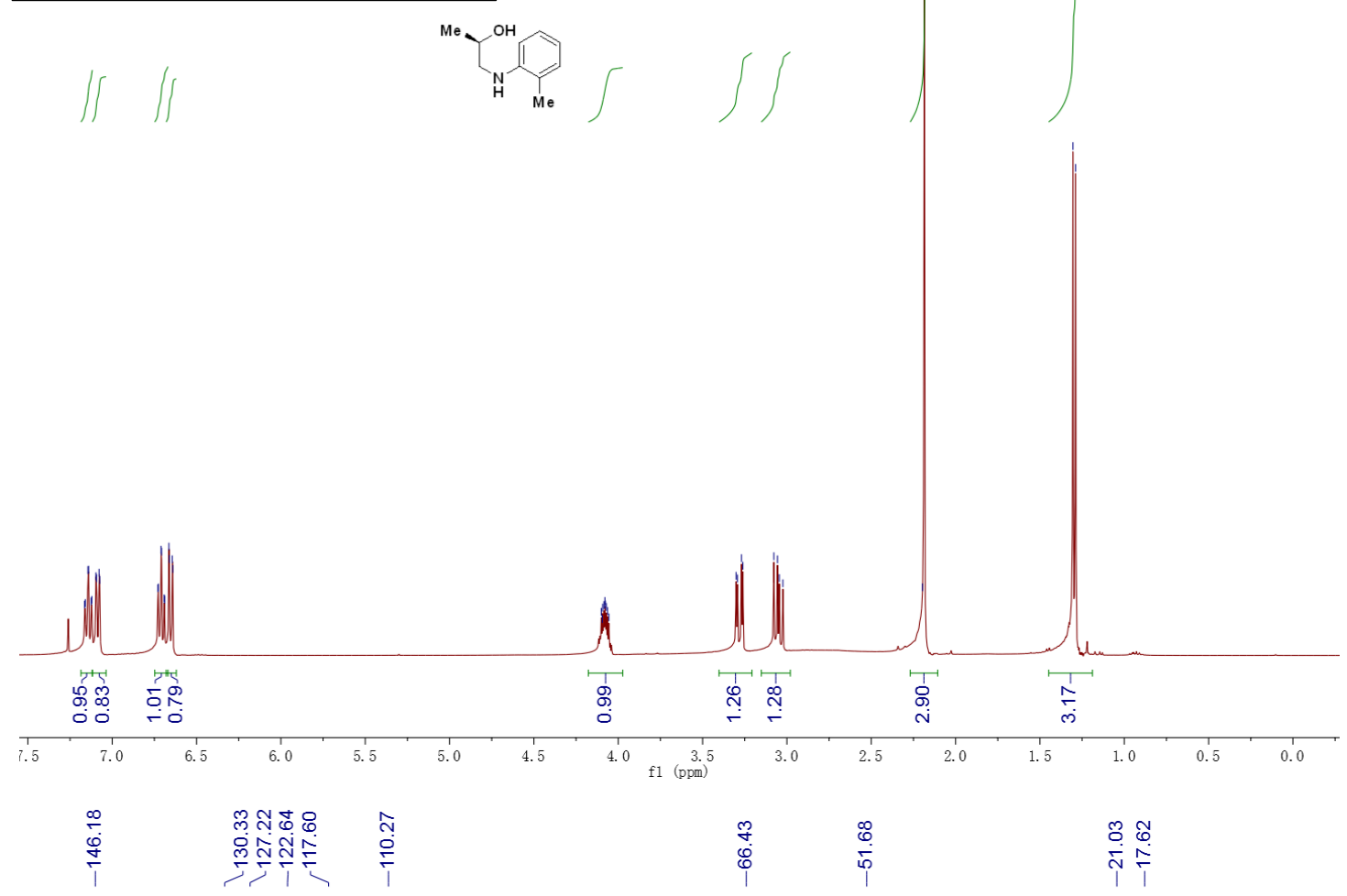

(R)-6, ${ }^{13} \mathrm{C} \mathrm{NMR}\left(101 \mathrm{MHz}, \mathrm{CDCl}_{3}\right)$
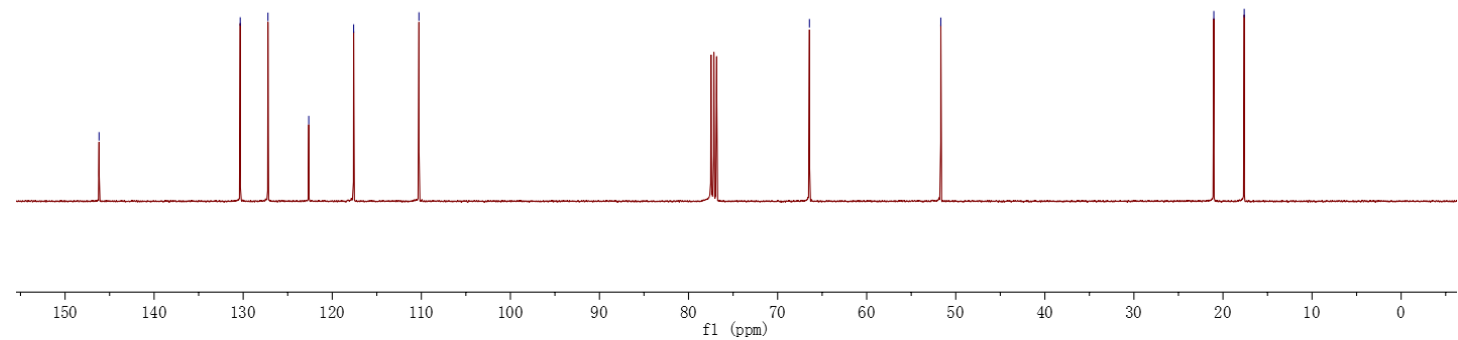


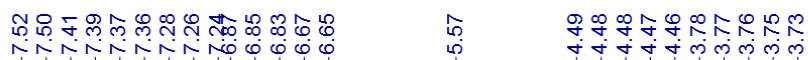

1a, ${ }^{1} \mathrm{H}$ NMR $\left(400 \mathrm{MHz}, \mathrm{CDCl}_{3}\right)$
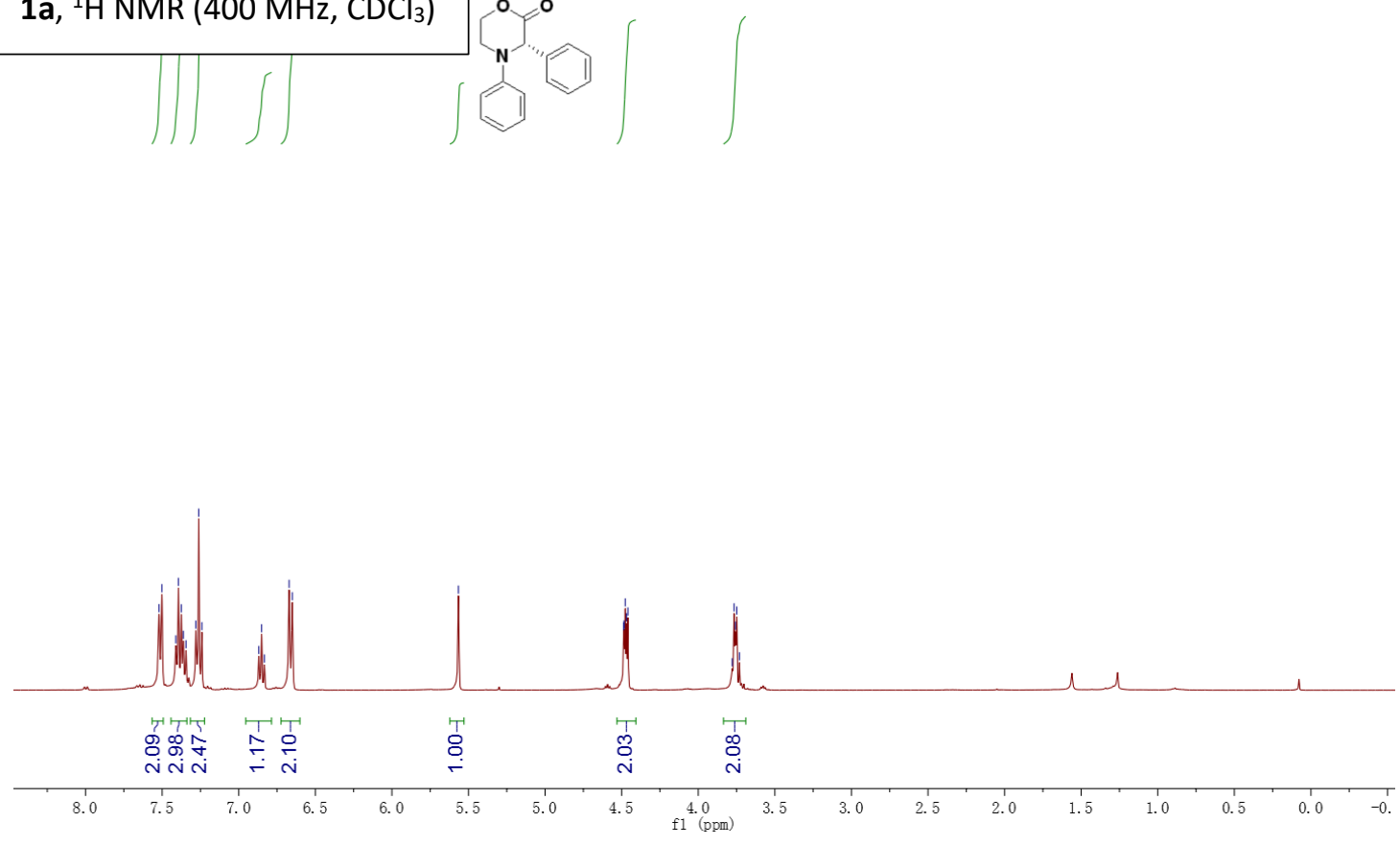

\begin{tabular}{|c|c|c|c|c|c|}
\hline $\begin{array}{l}5 \\
i \infty \\
0 \\
0\end{array}$ & 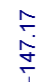 & 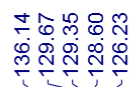 & & $\underset{\stackrel{N}{N}}{\stackrel{N}{\leftarrow}}$ & 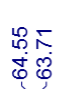 \\
\hline
\end{tabular}

1a, ${ }^{13} \mathrm{C}$ NMR (101 MHz, $\left.\mathrm{CDCl}_{3}\right)$

${ }_{N}^{0} Y^{0}$

1)

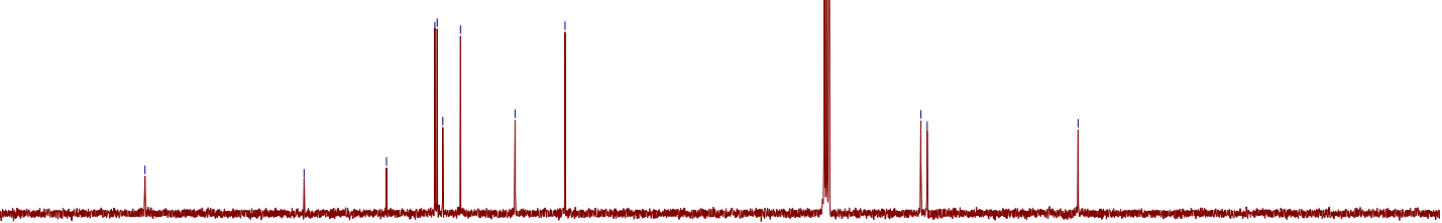

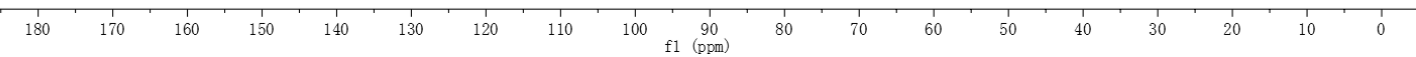




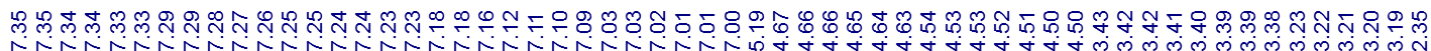

$\int_{\mathrm{H}_{3} \mathrm{C}}$

1b, ${ }^{1} \mathrm{H}$ NMR $\left(400 \mathrm{MHz}^{\mathrm{CDCl}} 3\right)$

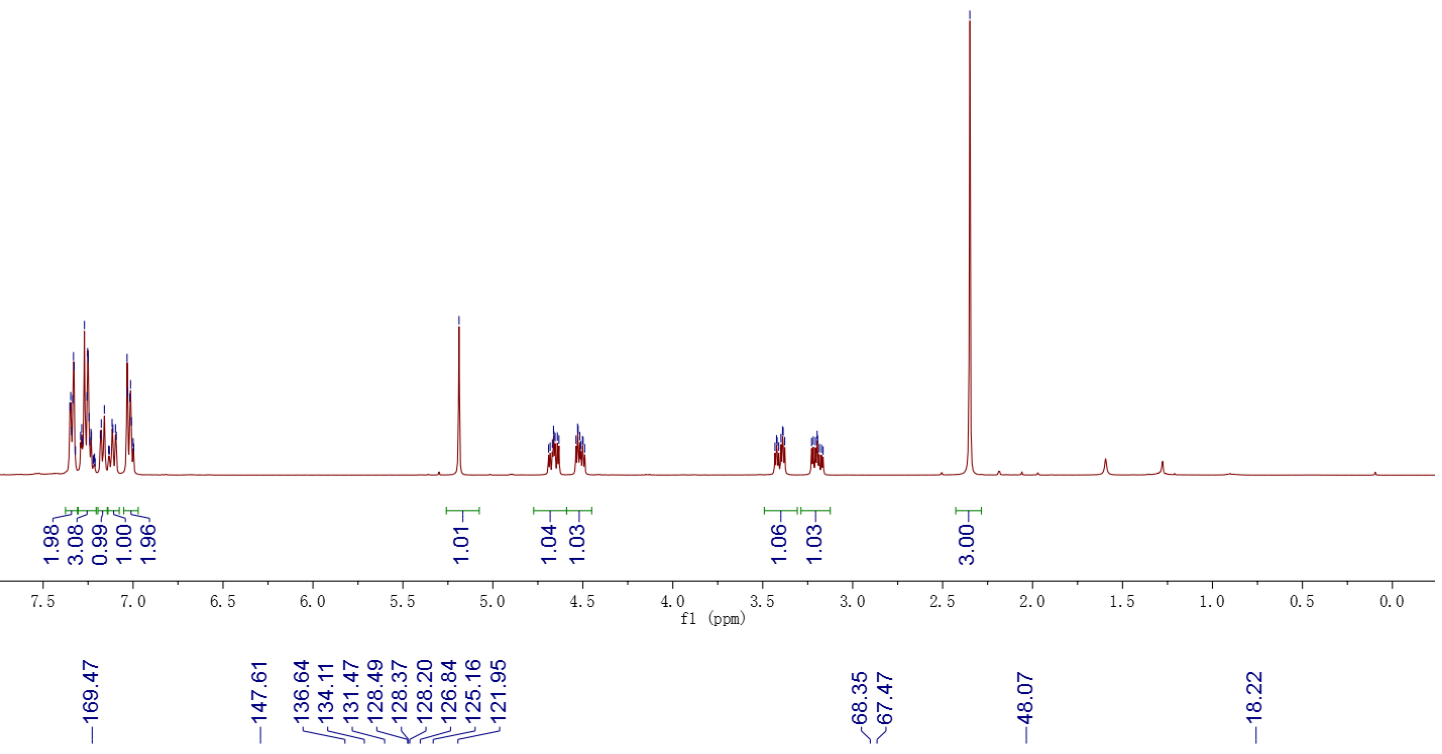

1b, $\left.{ }^{13} \mathrm{C} \mathrm{NMR} \mathrm{(101} \mathrm{MHz,} \mathrm{CDCl}_{3}\right)$

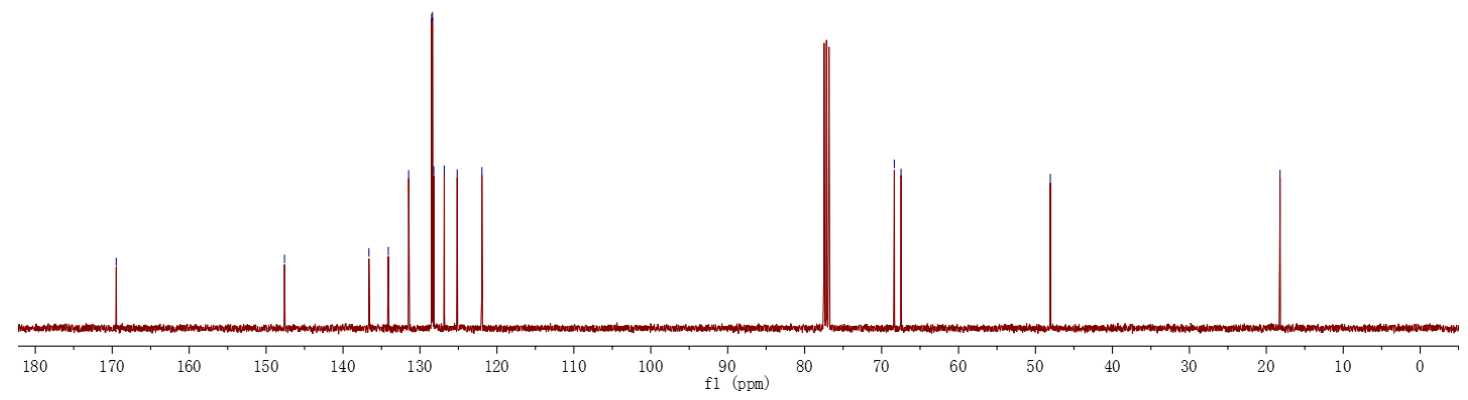




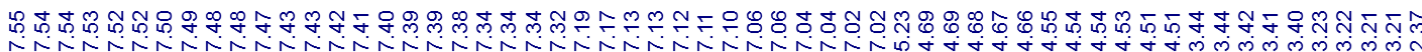
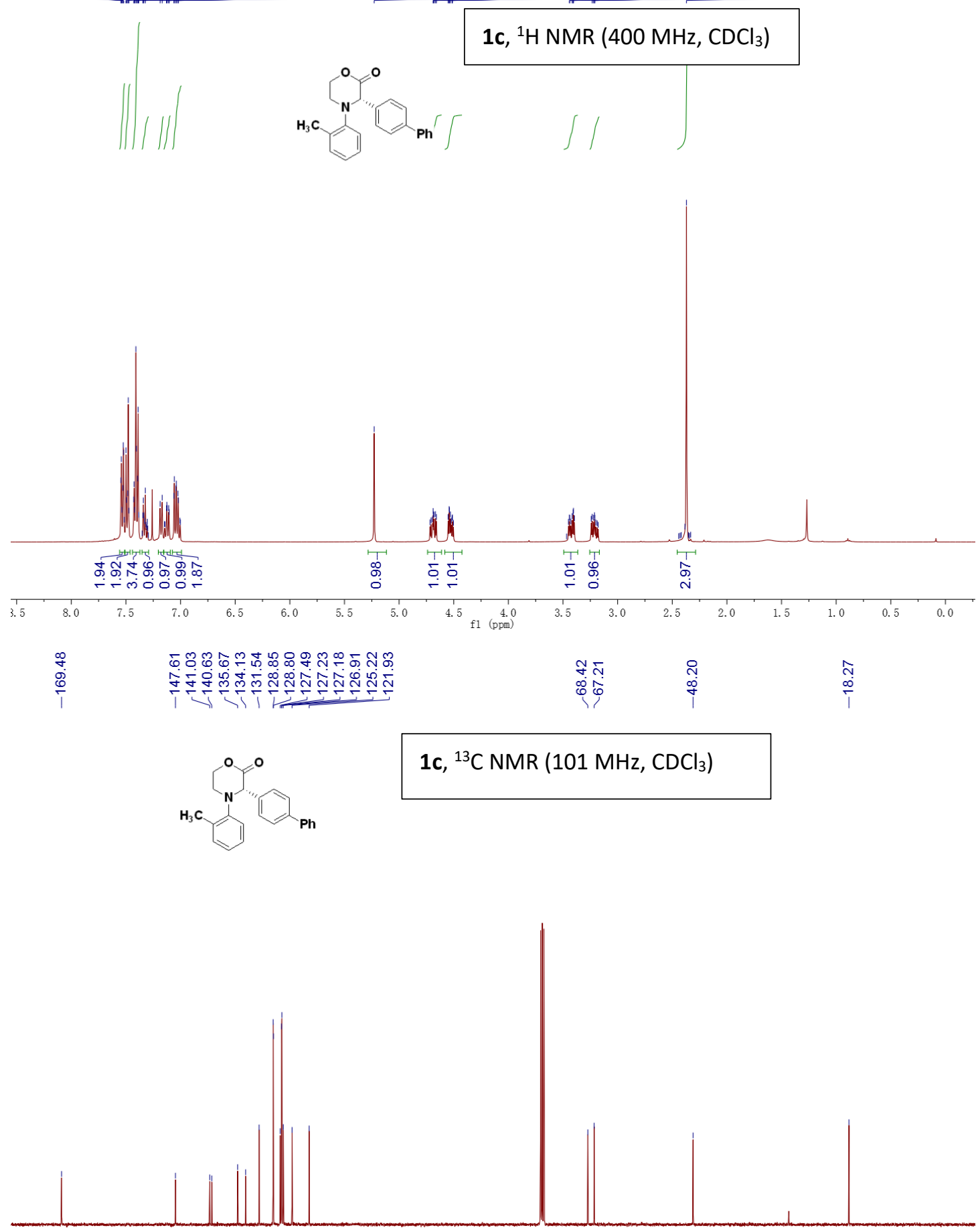

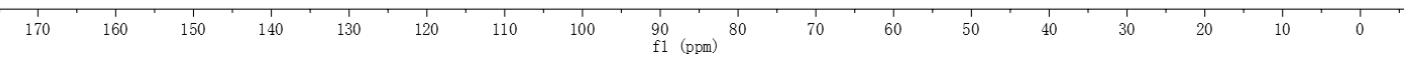




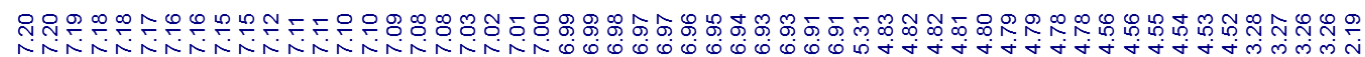

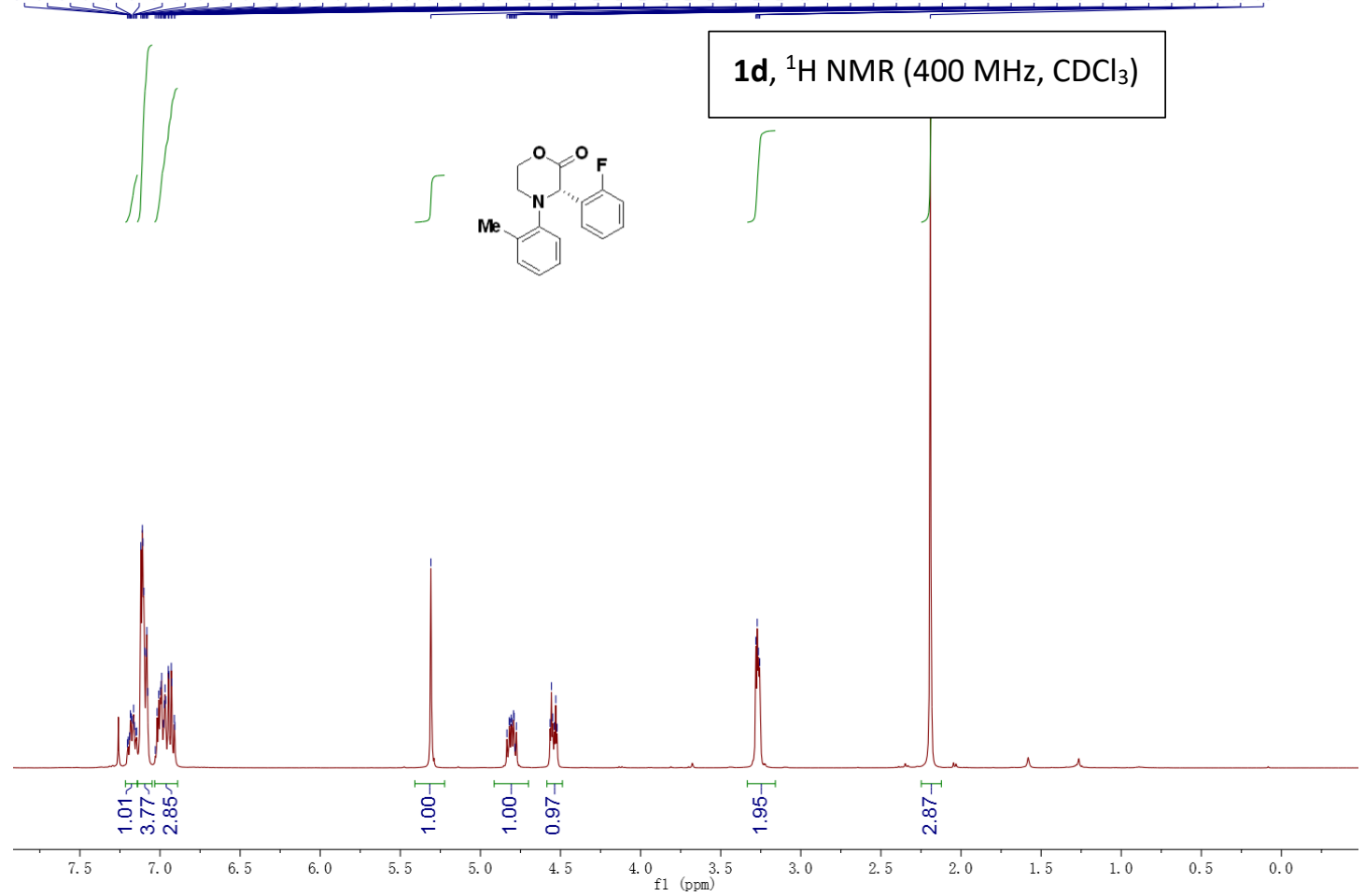

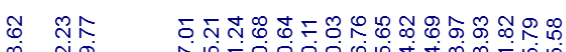

i

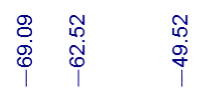

ำ

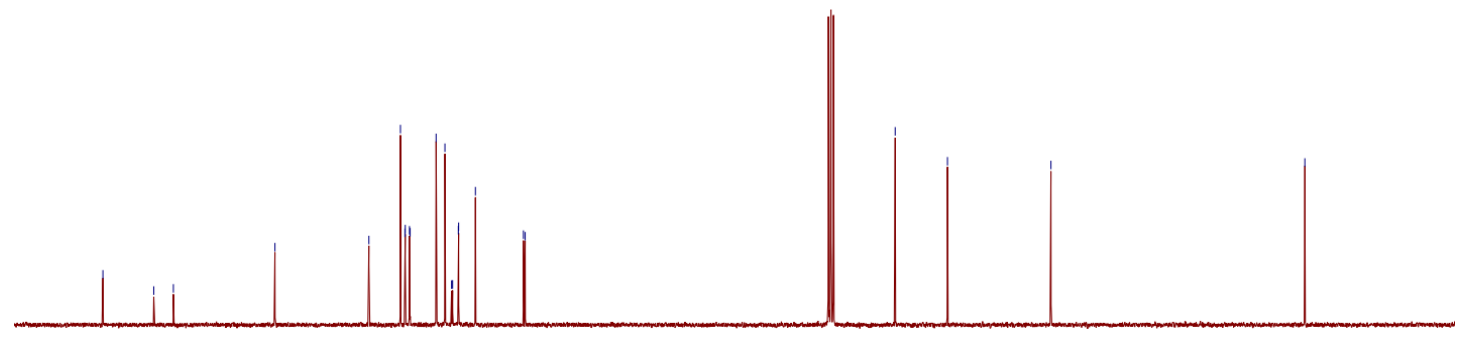

1d, ${ }^{13} \mathrm{C} \mathrm{NMR}\left(101 \mathrm{MHz}, \mathrm{CDCl}_{3}\right)$

Me

(

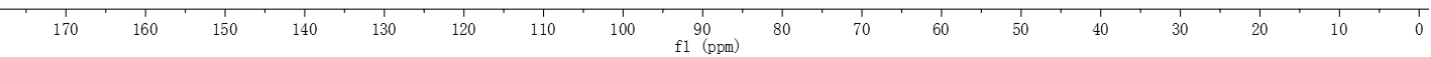




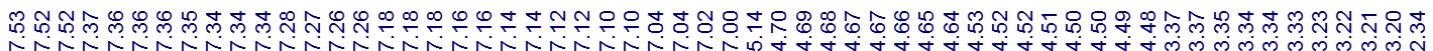

1e, ${ }^{1} \mathrm{H}$ NMR $\left(400 \mathrm{MHz} \mathrm{CDCl}_{3}\right)$
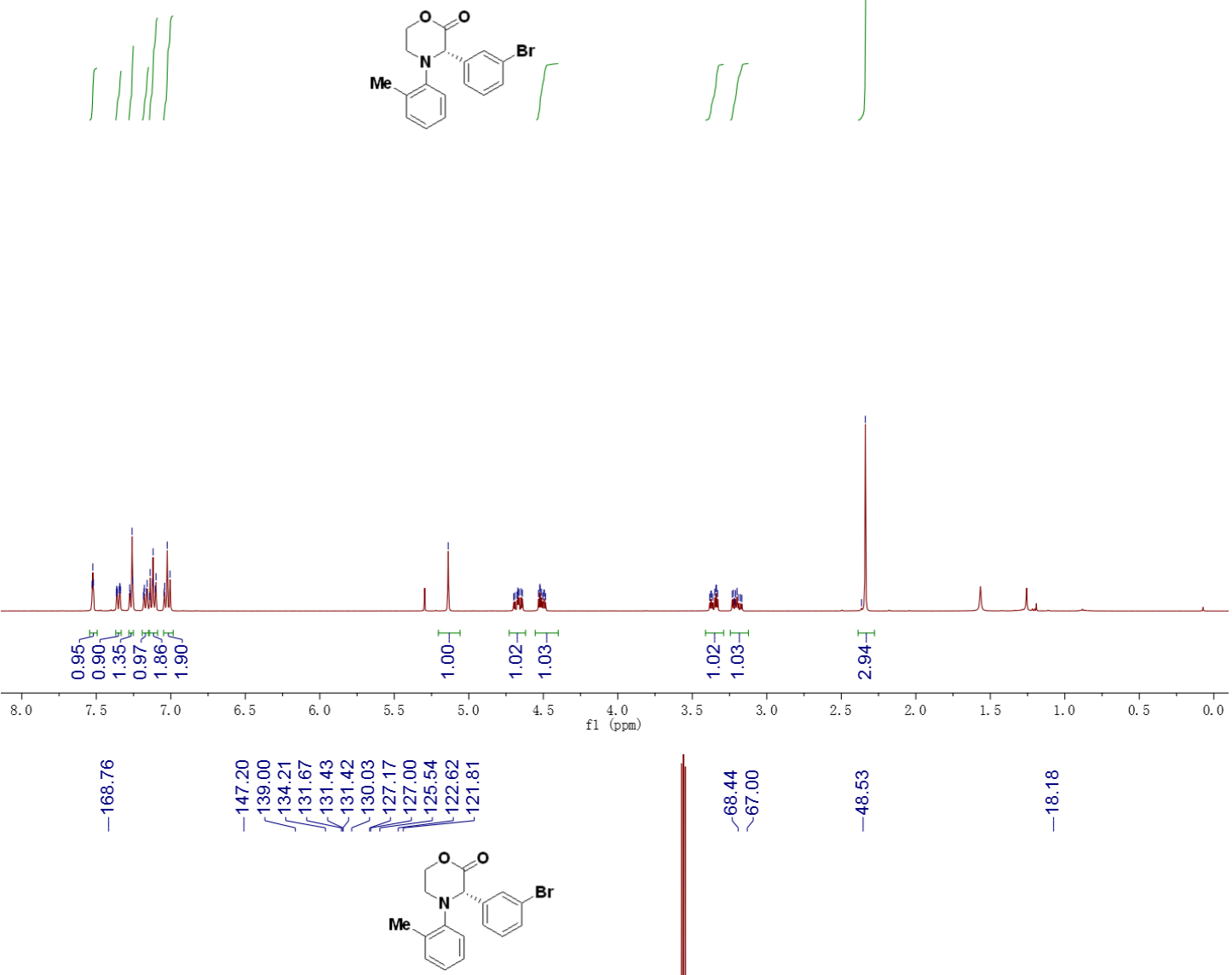

1e, ${ }^{13} \mathrm{C}$ NMR $\left(101 \mathrm{MHz}, \mathrm{CDCl}_{3}\right)$
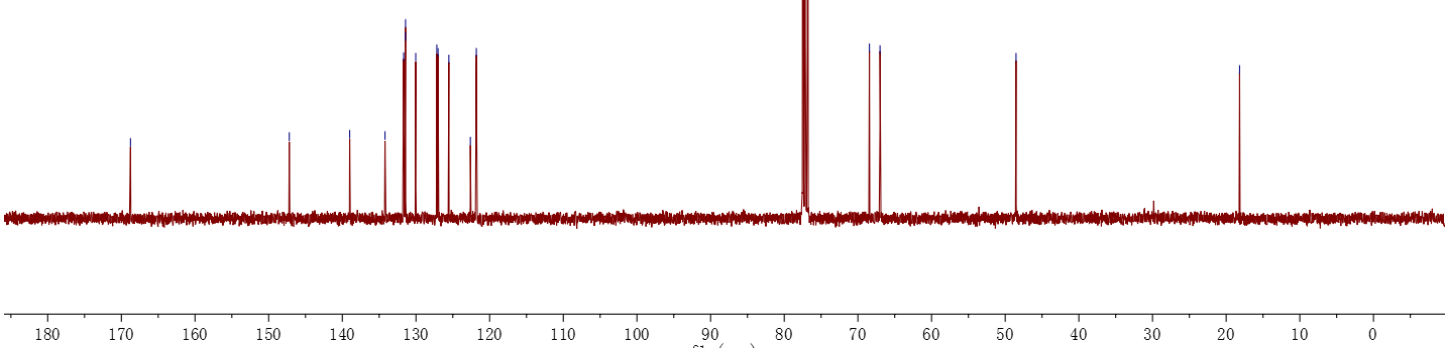

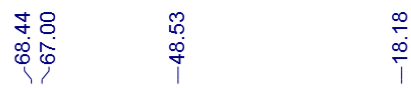

$\mathrm{fl}^{90}(\mathrm{ppm}) \stackrel{80}{ }$ 


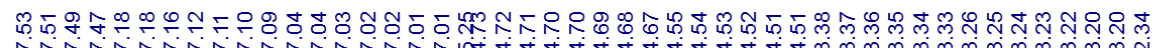

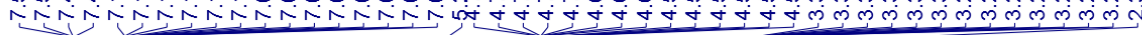

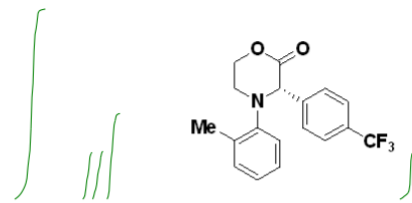

1f, ${ }^{1} \mathrm{H}$ NMR $\left(400 \mathrm{MHz}, \mathrm{CDCl}_{3}\right)$
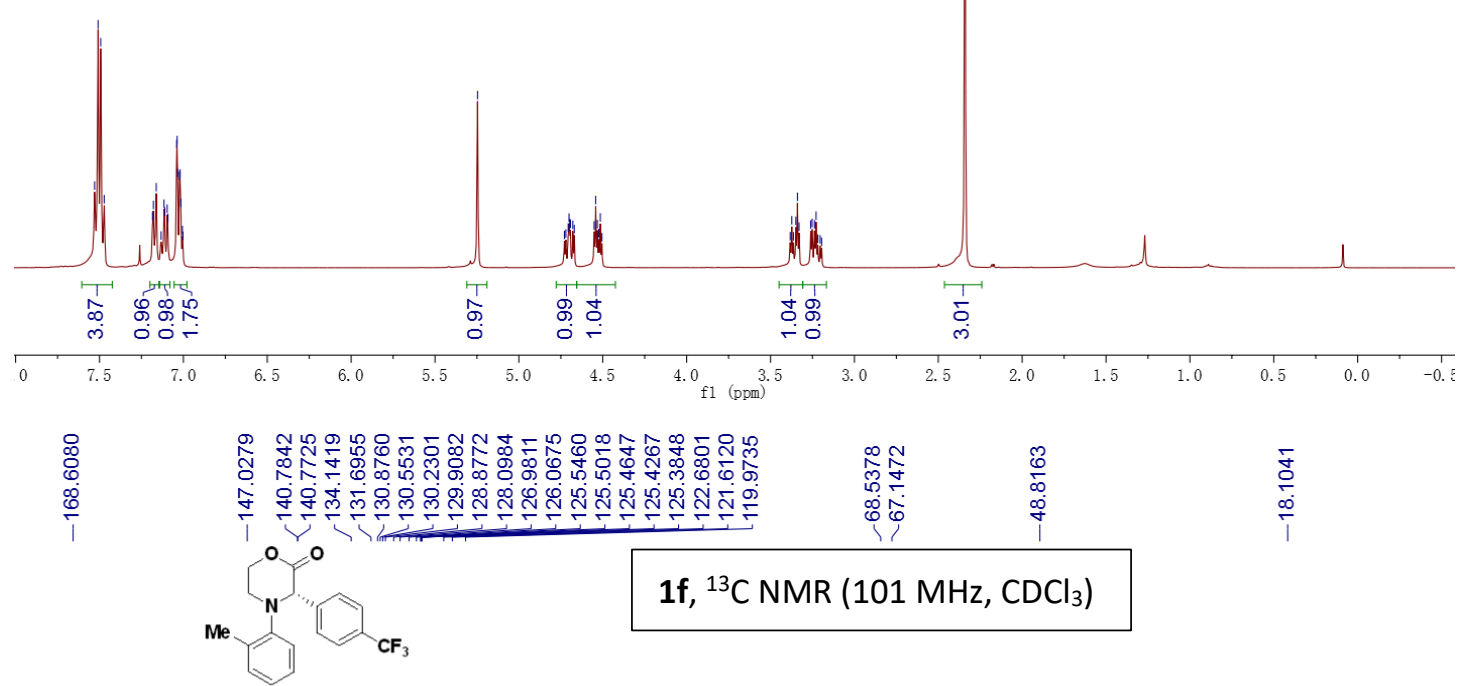

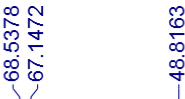

$\frac{5}{\frac{5}{0}}$

1f, ${ }^{13} \mathrm{C}$ NMR (101 MHz, $\left.\mathrm{CDCl}_{3}\right)$

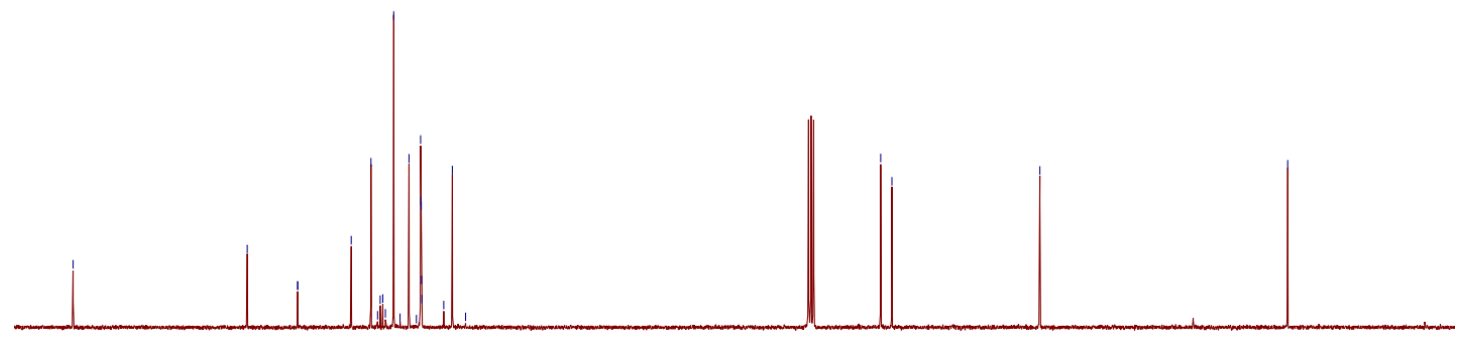

170

160

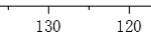

${ }_{\mathrm{f} 1(\mathrm{ppm})}^{80}$ 


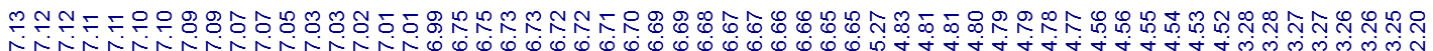
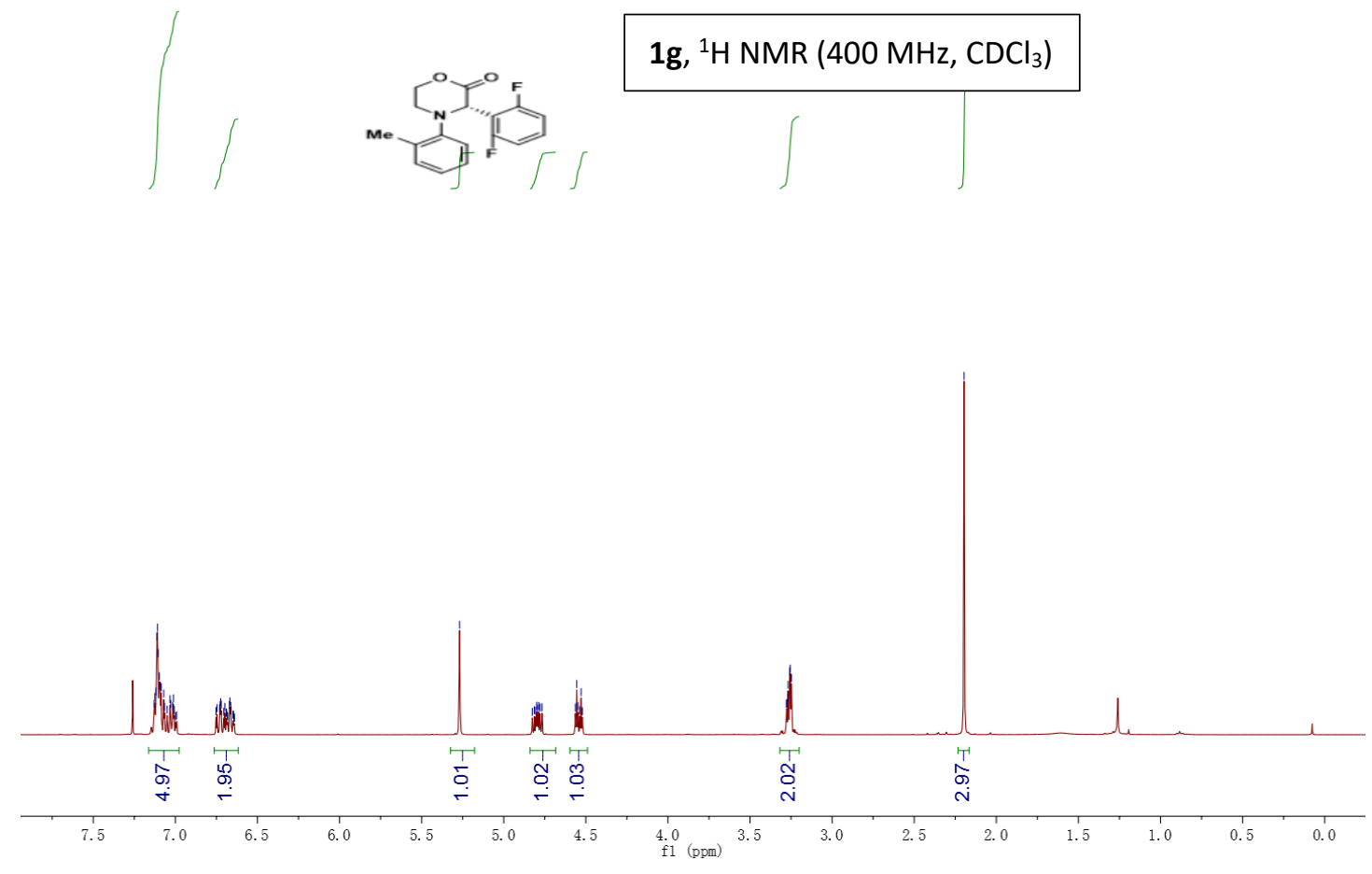

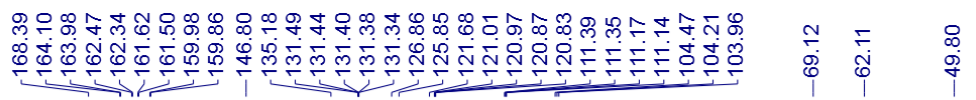

1g, ${ }^{13} \mathrm{C}$ NMR $\left(101 \mathrm{MHz}, \mathrm{CDCl}_{3}\right)$

$\operatorname{me}$

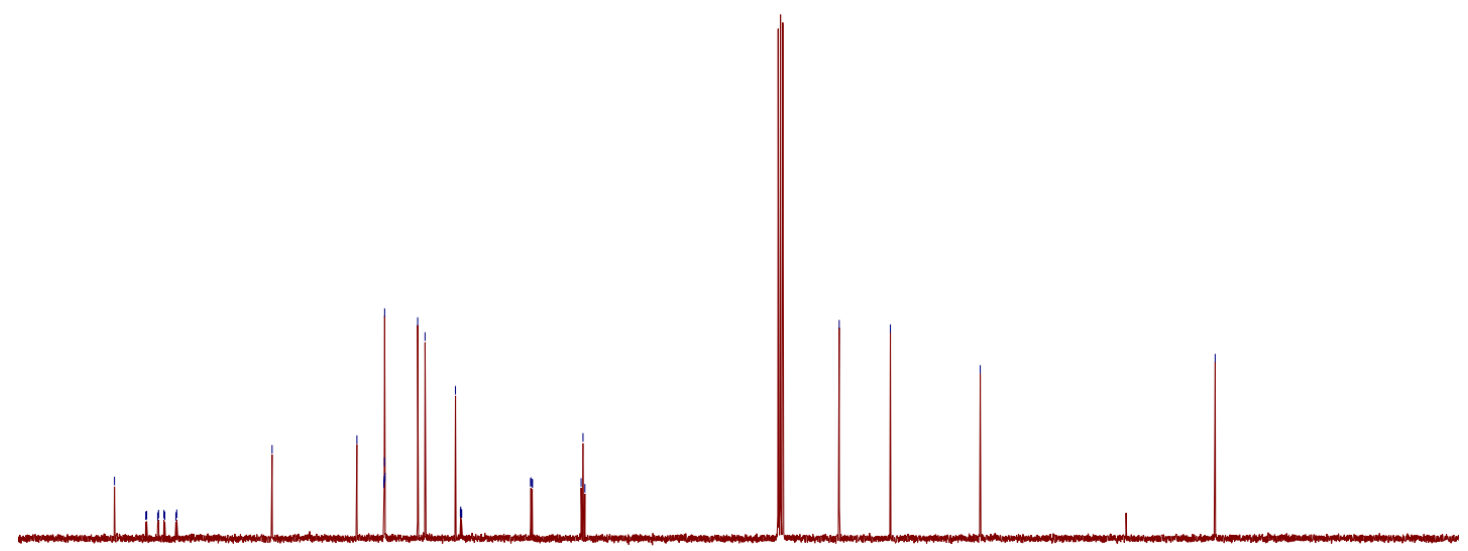

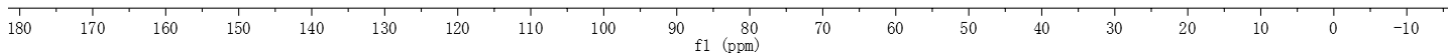


1h, ${ }^{1} \mathrm{H}$ NMR $\left(400 \mathrm{MHz}, \mathrm{CDCl}_{3}\right)$<smiles>COC1COCCN(c2ccccc2C)[C@@H](c2ccc3ccccc3c2)C1=O</smiles>
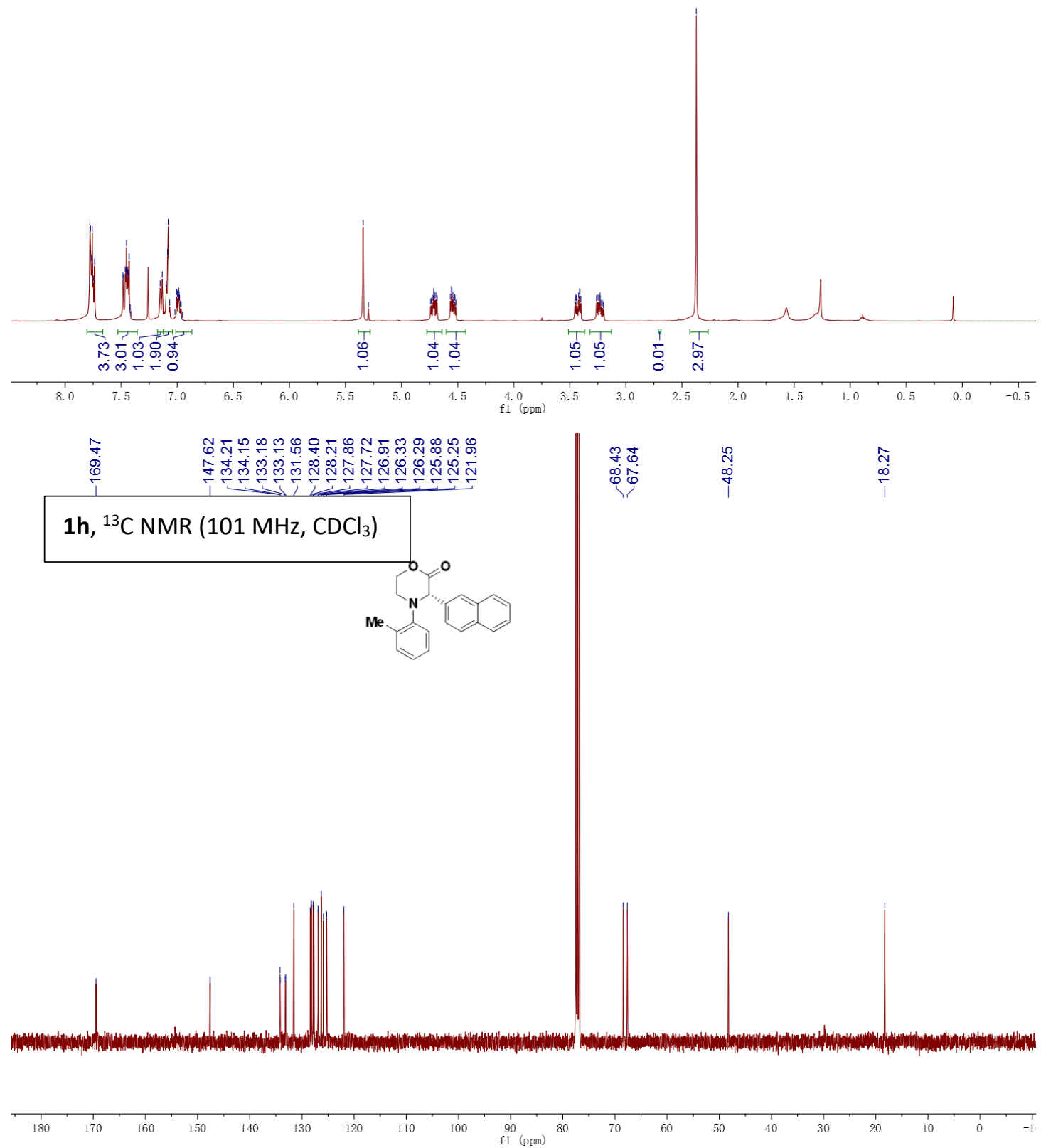


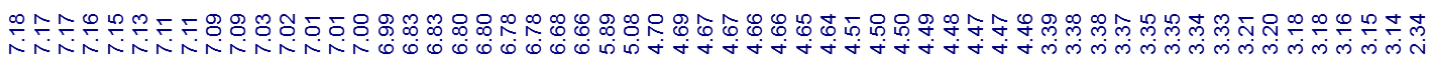

1i, ${ }^{1} \mathrm{H}$ NMR (400 MHz, $\mathrm{CDCl}_{3}$ )

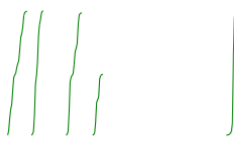

$\mathrm{me}$
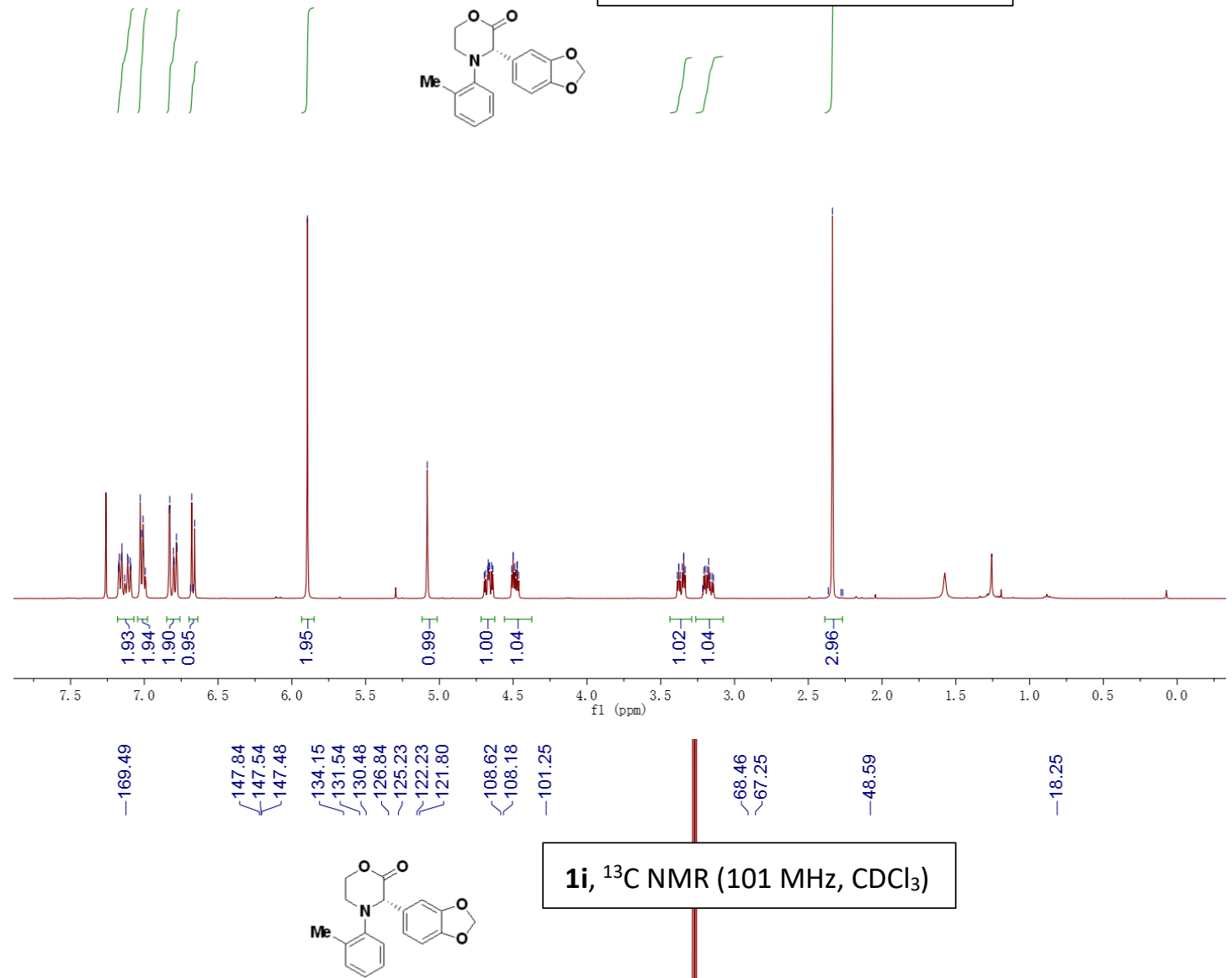

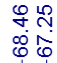

$\stackrel{\infty}{\infty}$
$\infty$
+
+

$\stackrel{\stackrel{n}{N}}{\stackrel{\infty}{\infty}}$

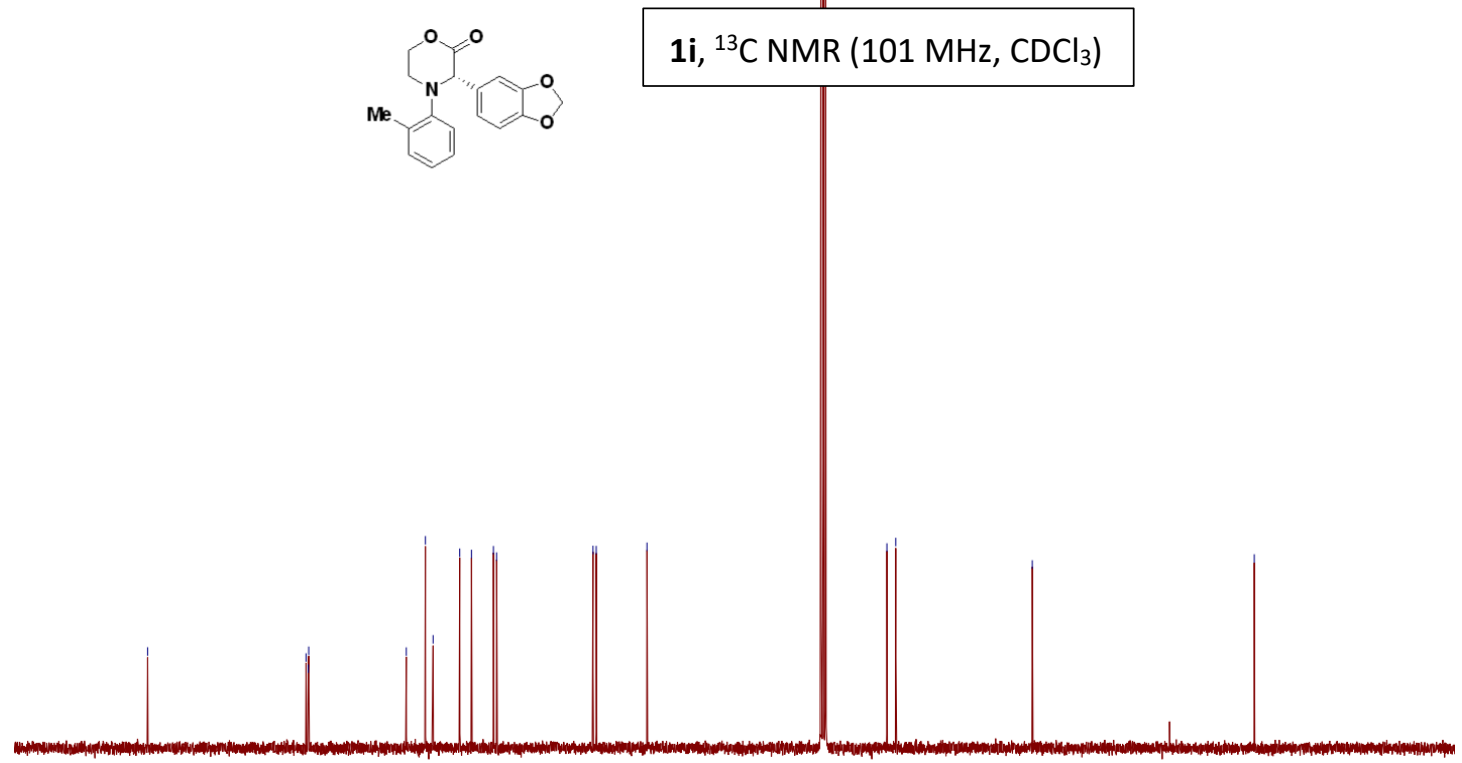

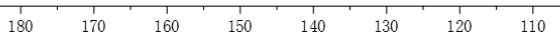
$\mathrm{f} 1 \mathrm{90}(\mathrm{ppm})$ 


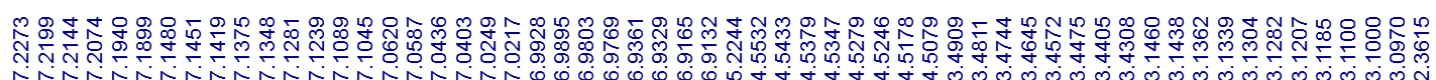

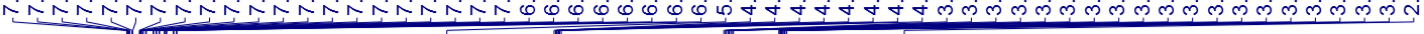
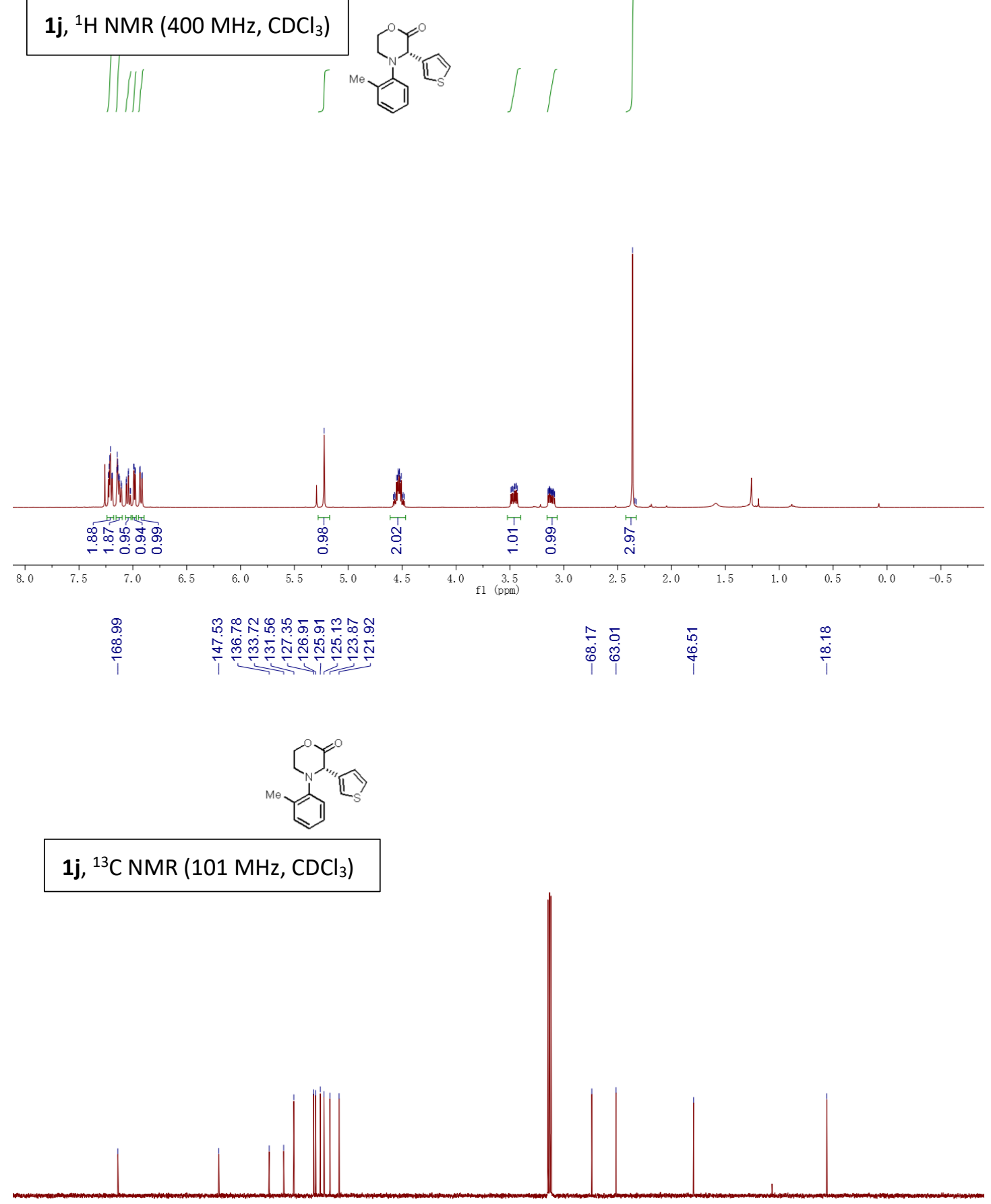

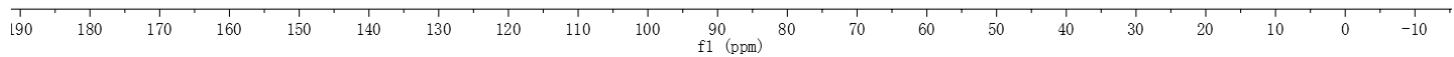




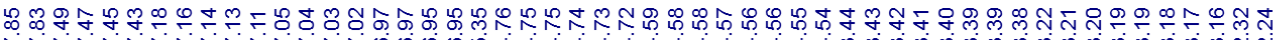

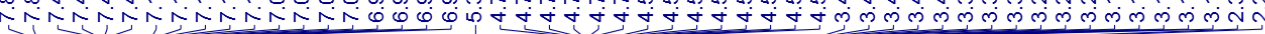

1k, ${ }^{1} \mathrm{H}$ NMR $\left(400 \mathrm{MHz}, \mathrm{CDCl}_{3}\right)$

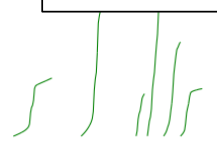

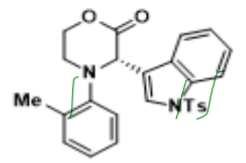
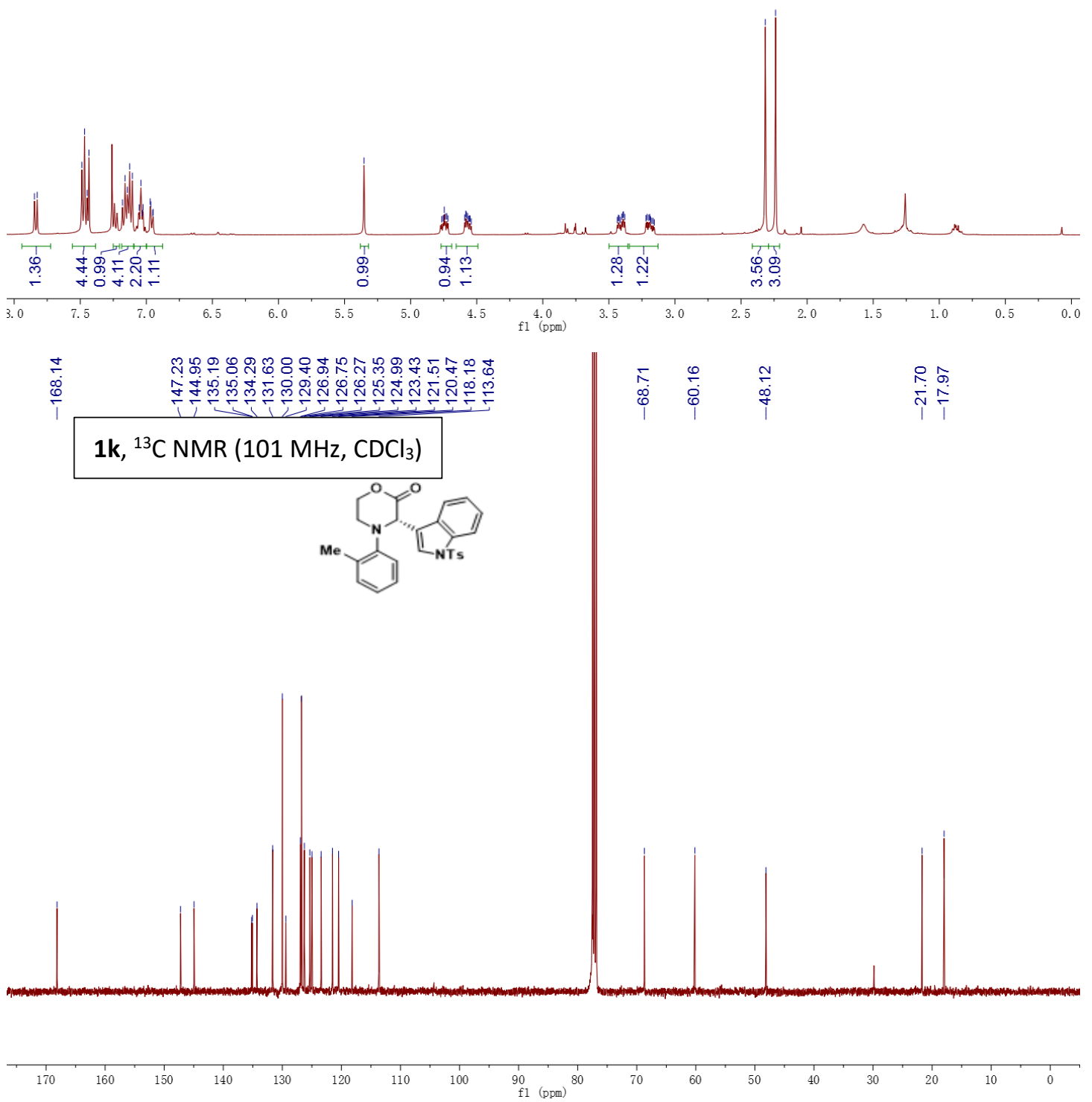


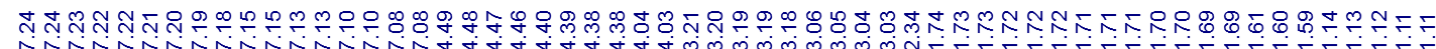

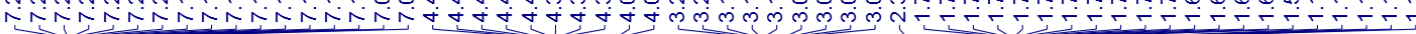

1I, ${ }^{1} \mathrm{H}$ NMR $\left(400 \mathrm{MHz}, \mathrm{CDCl}_{3}\right)$
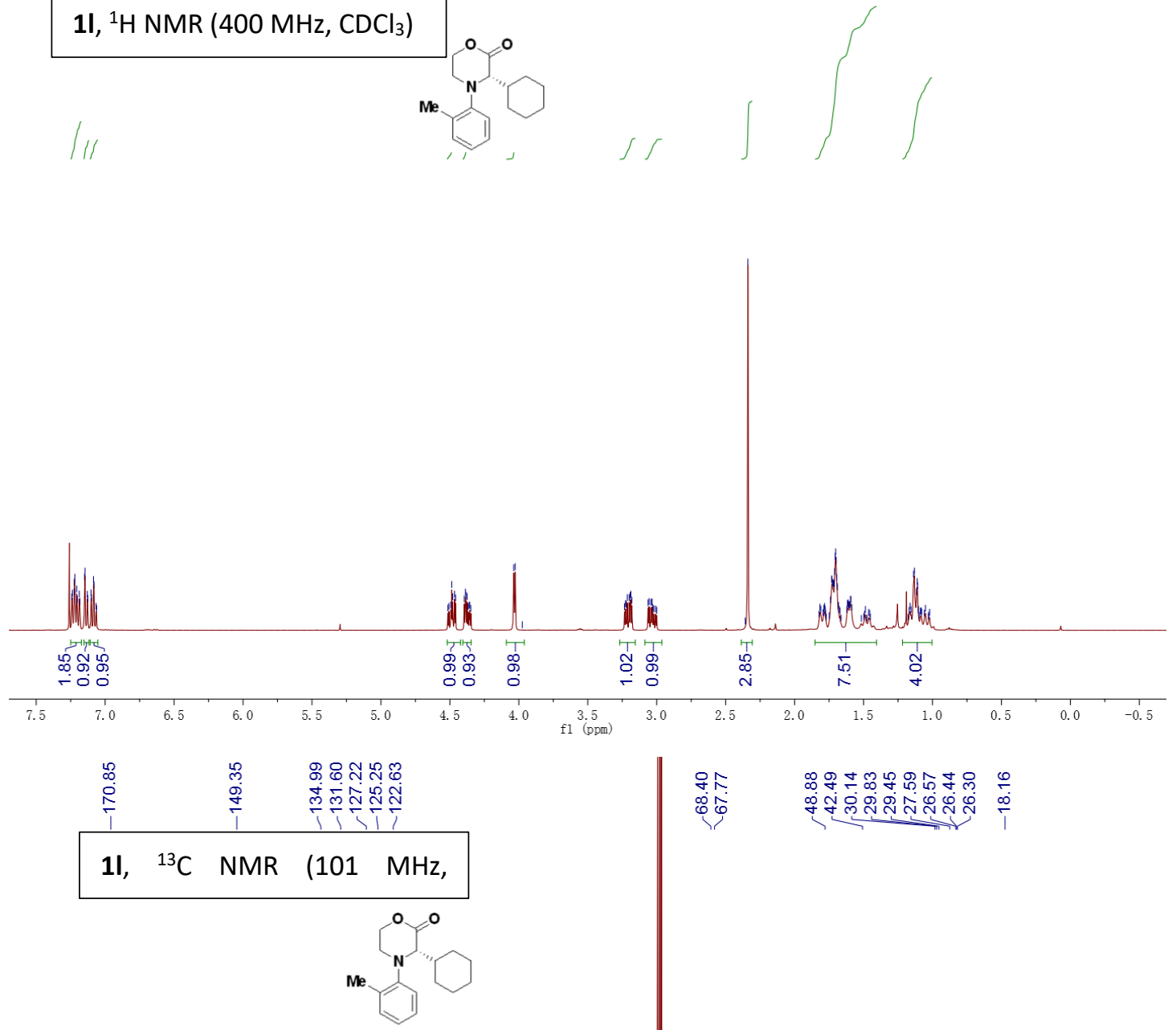

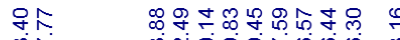

il

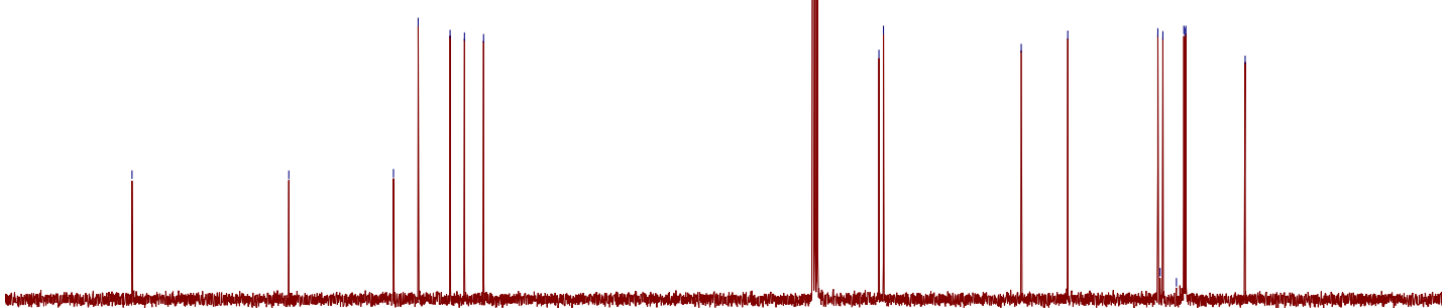

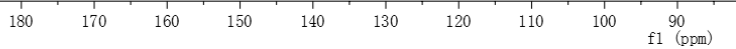




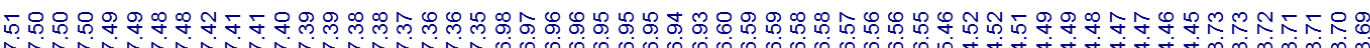

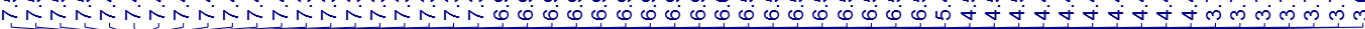
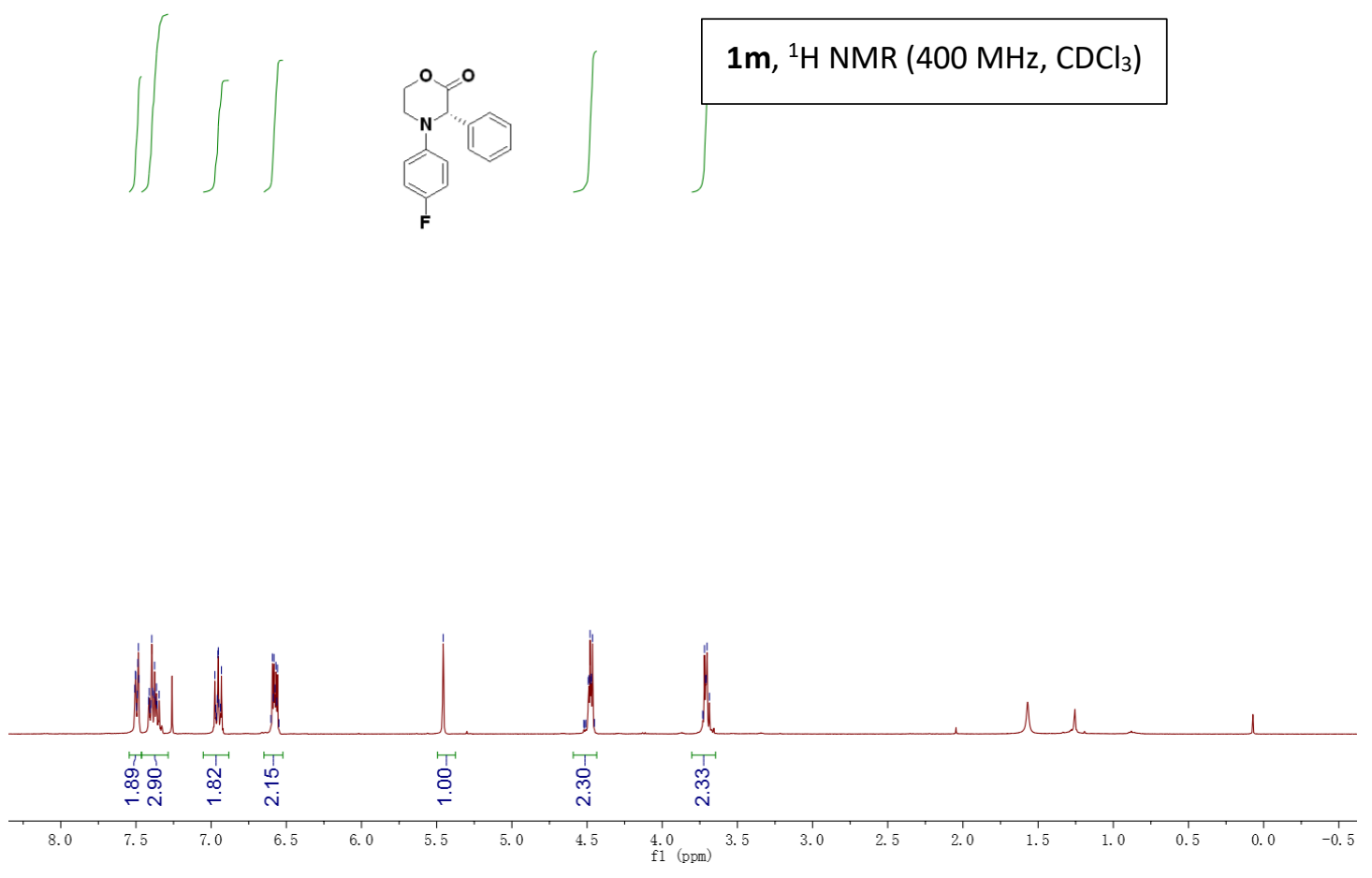

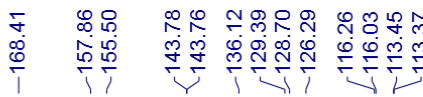

ली

$1 \mathrm{~m},{ }^{13} \mathrm{C}$ NMR $\left(101 \mathrm{MHz}, \mathrm{CDCl}_{3}\right)$
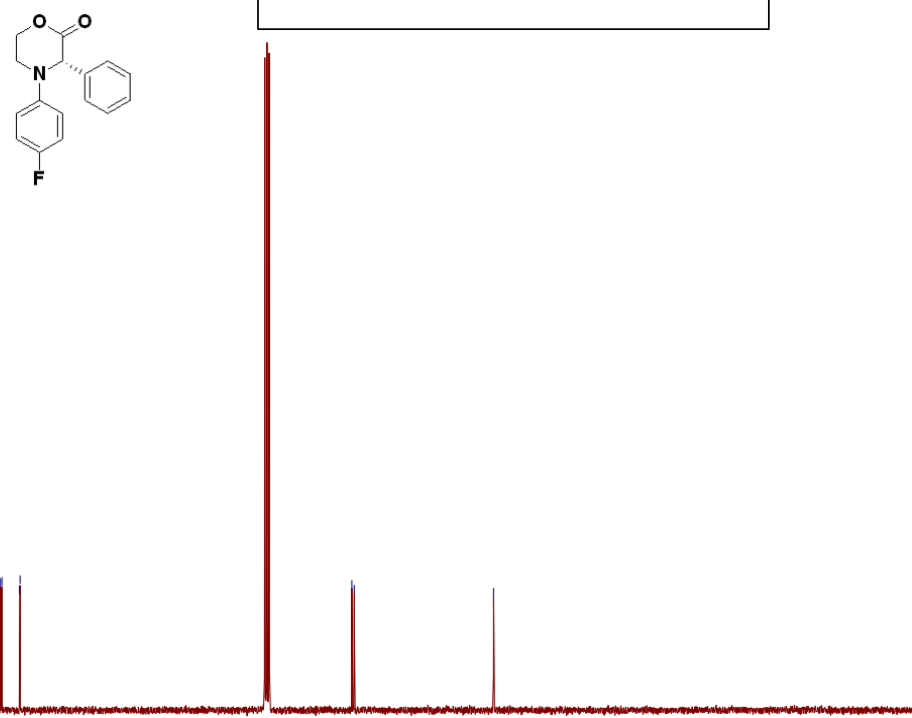

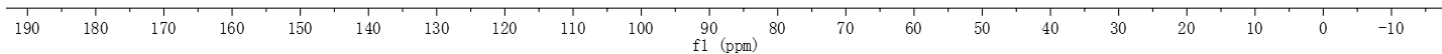




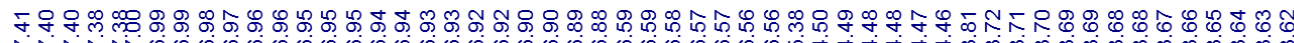

NiN Ni
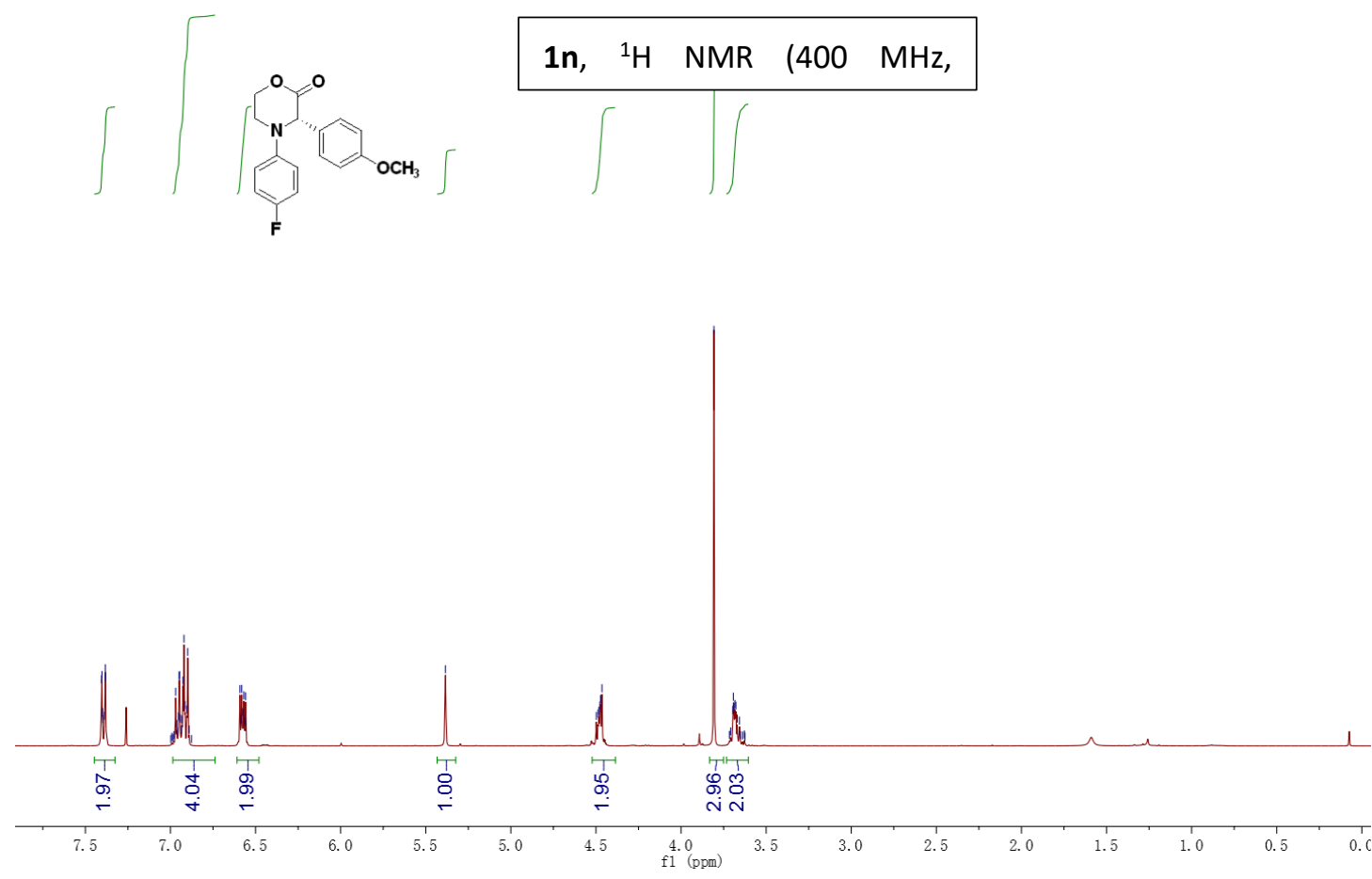

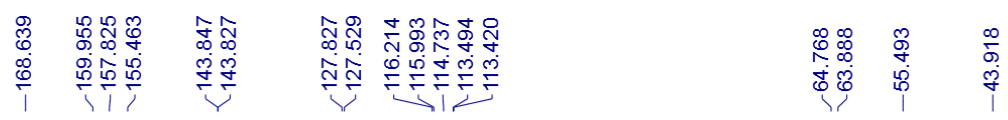

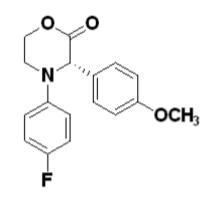

1n, ${ }^{13} \mathrm{C}$ NMR $\left(101 \mathrm{MHz}, \mathrm{CDCl}_{3}\right)$

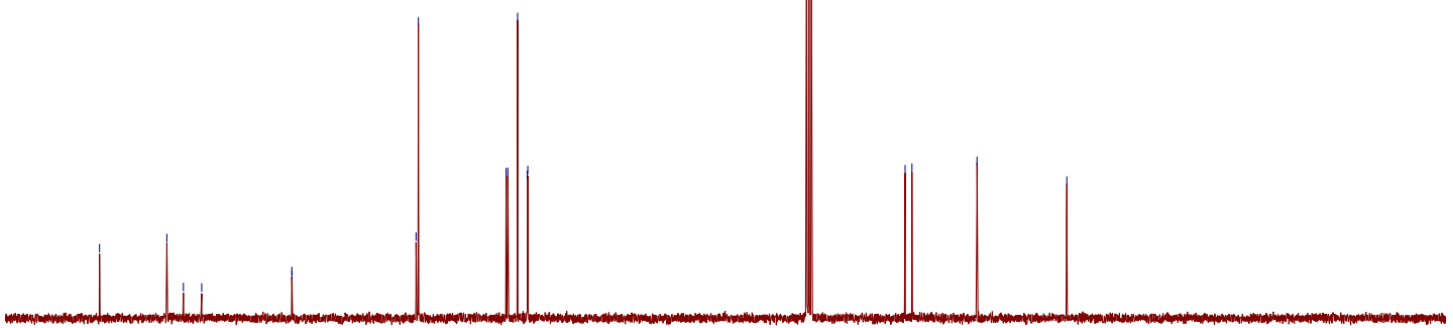

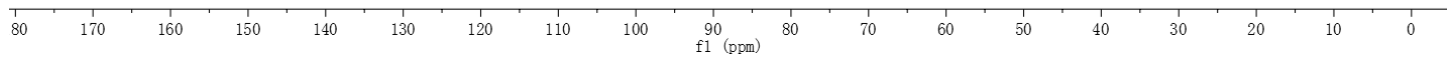




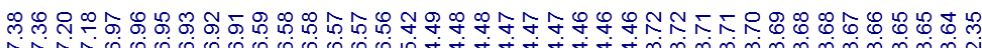

rí,

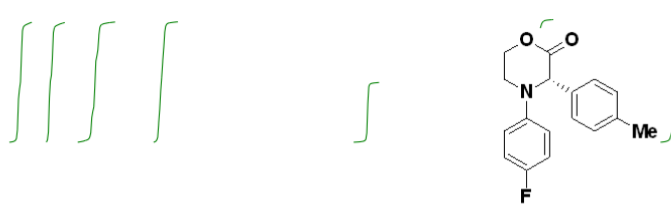

10, ${ }^{1} \mathrm{H}$ NMR (400 MHz, $\mathrm{CDCl}_{3}$ )

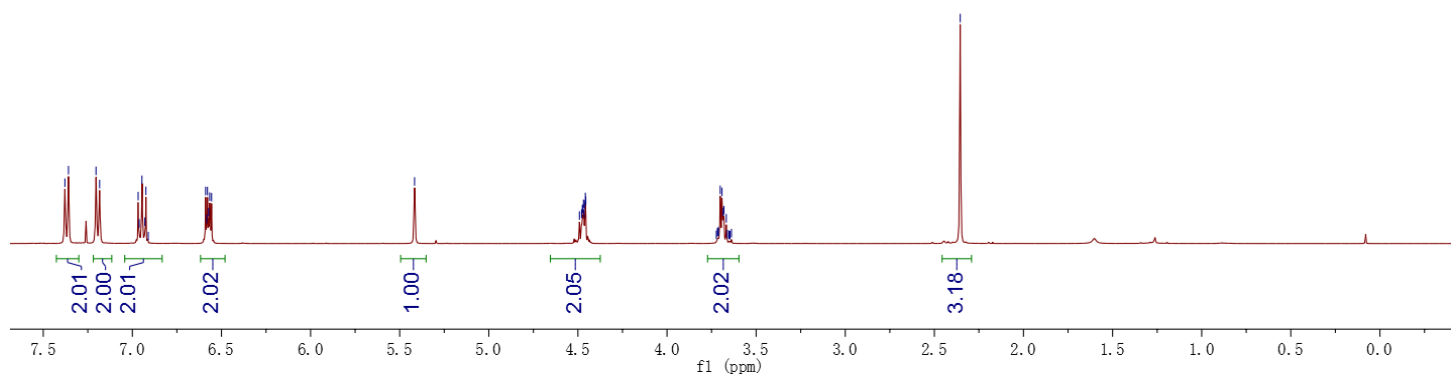

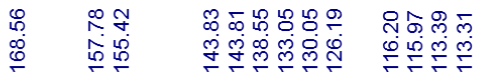
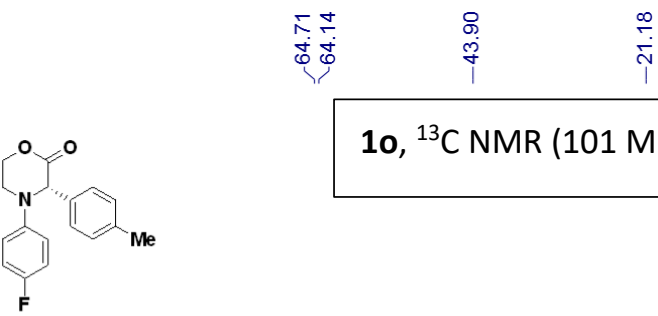

10, ${ }^{13} \mathrm{C}$ NMR $\left(101 \mathrm{MHz}, \mathrm{CDCl}_{3}\right)$

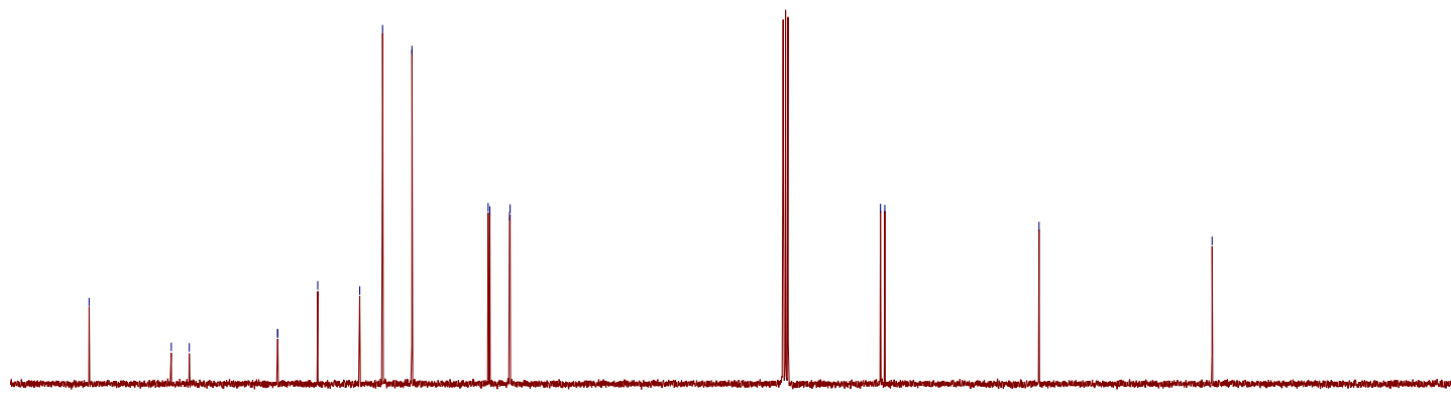

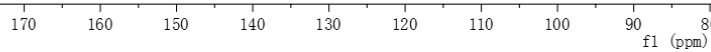




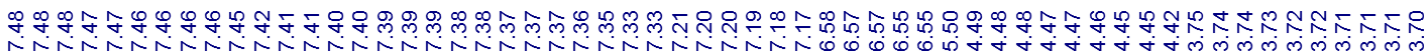

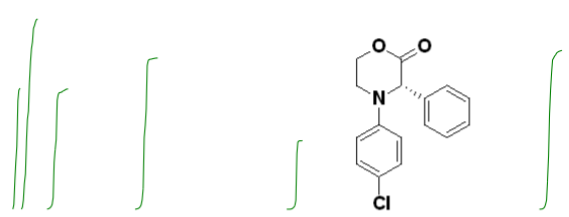

1p, ${ }^{1} \mathrm{H}$ NMR (400 MHz, $\mathrm{CDCl}_{3}$ )

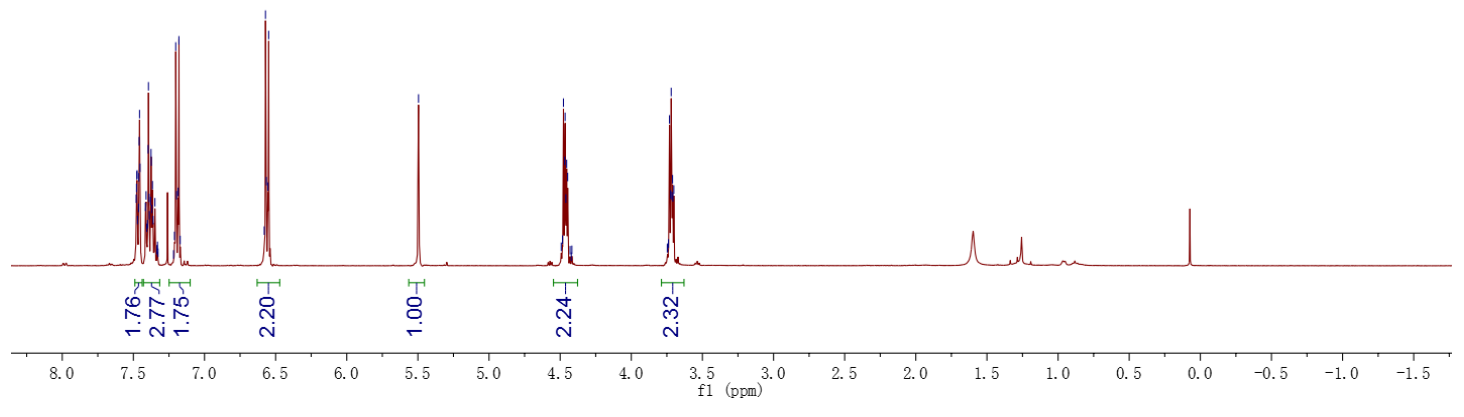

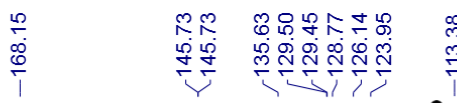

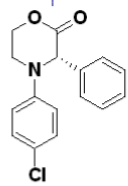

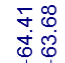

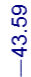

1p, ${ }^{13} \mathrm{C}$ NMR (101 MHz, $\left.\mathrm{CDCl}_{3}\right)$

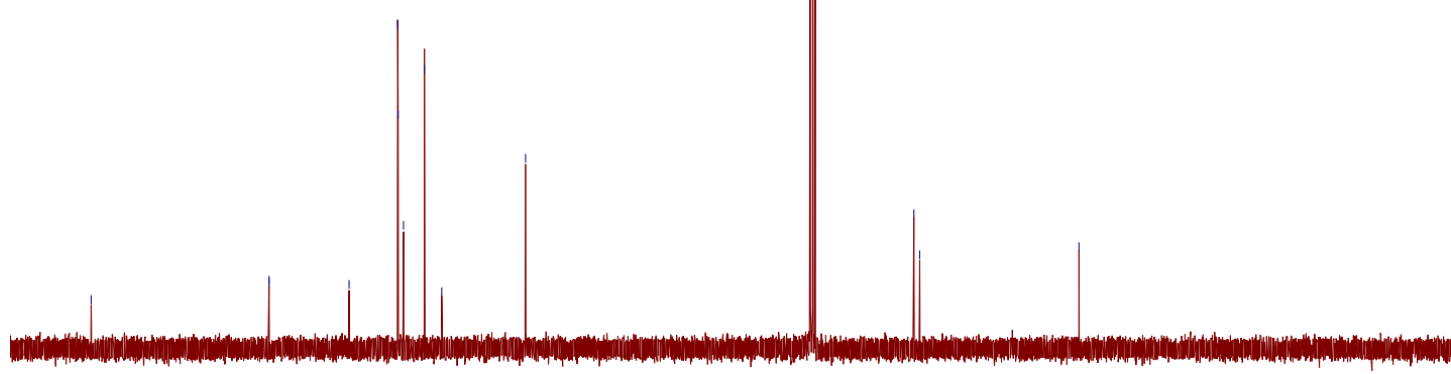

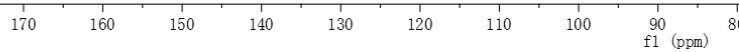

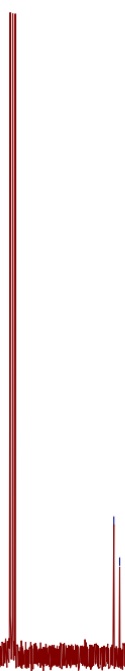




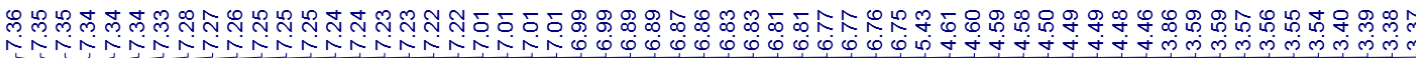
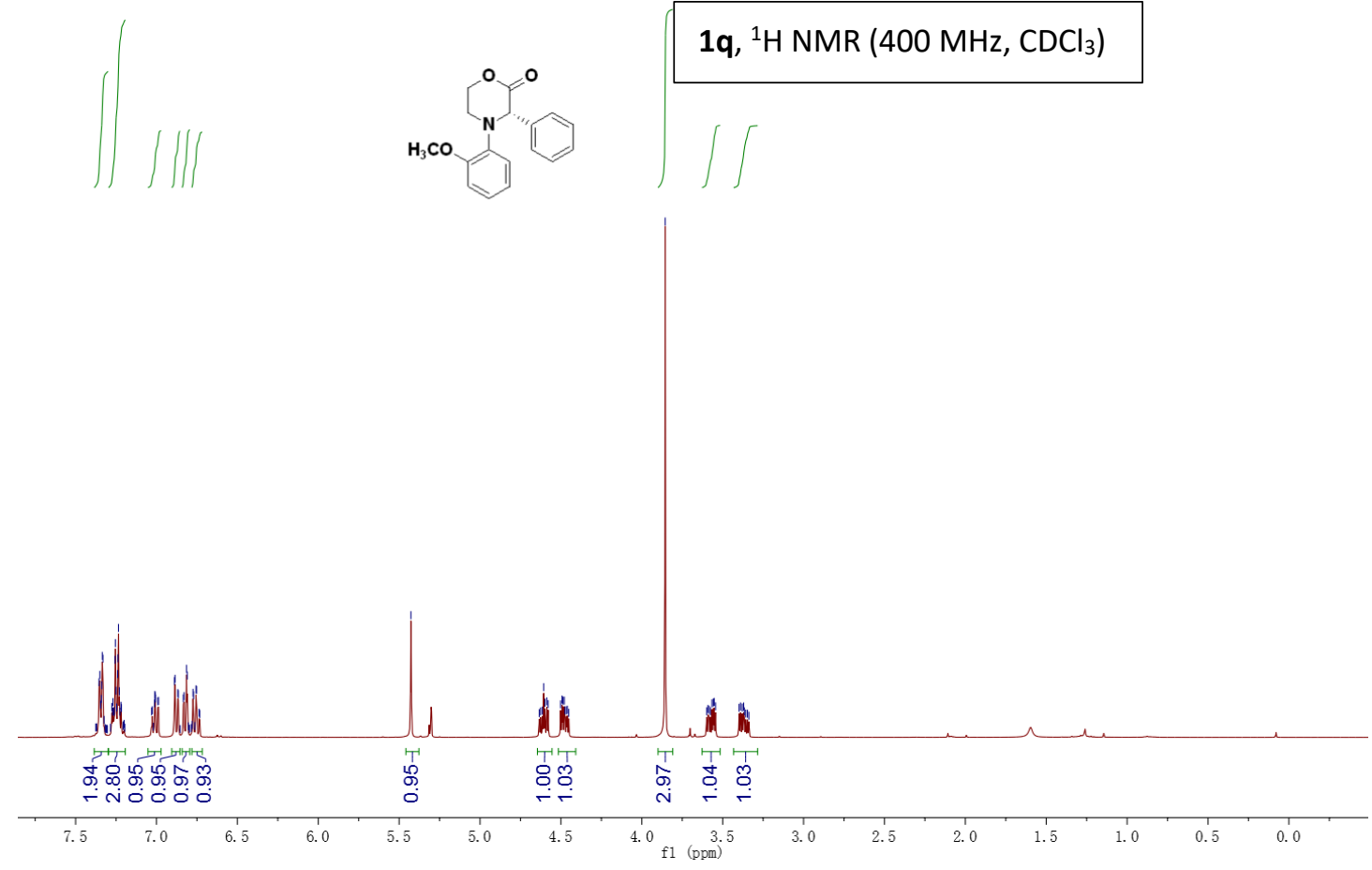

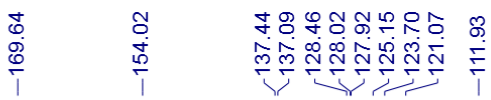

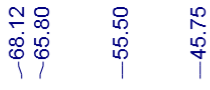

$\mathrm{H}_{3} \mathrm{CO}$

1q, ${ }^{13} \mathrm{C} \quad \mathrm{NMR} \quad(101 \mathrm{MHz}$

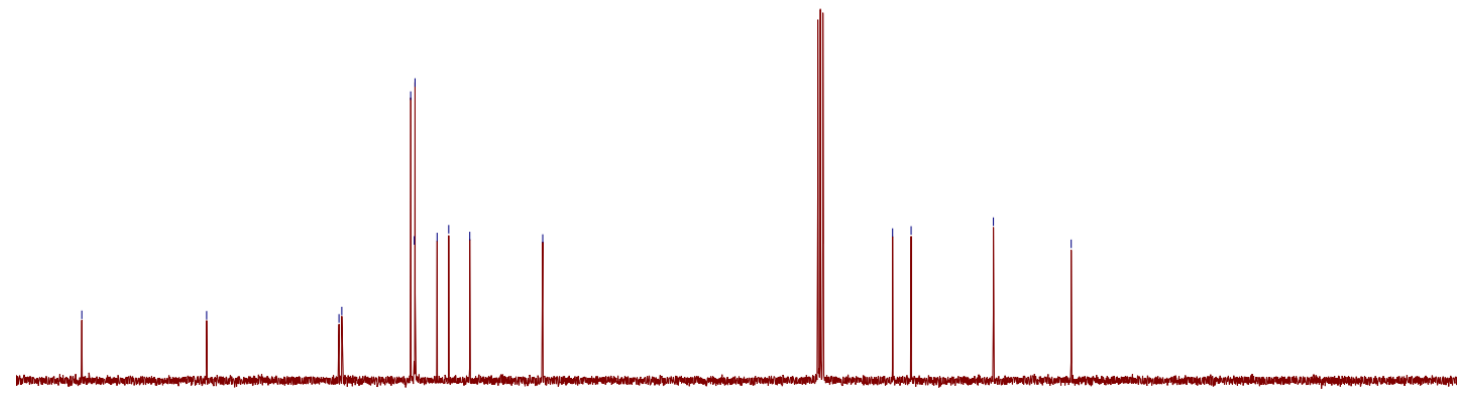

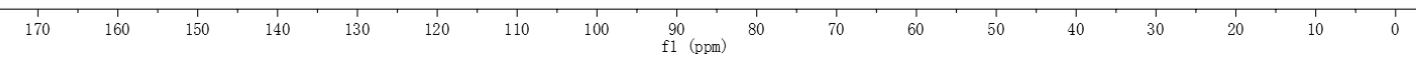




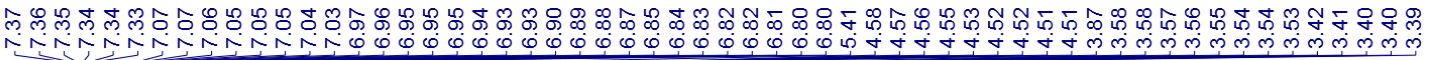
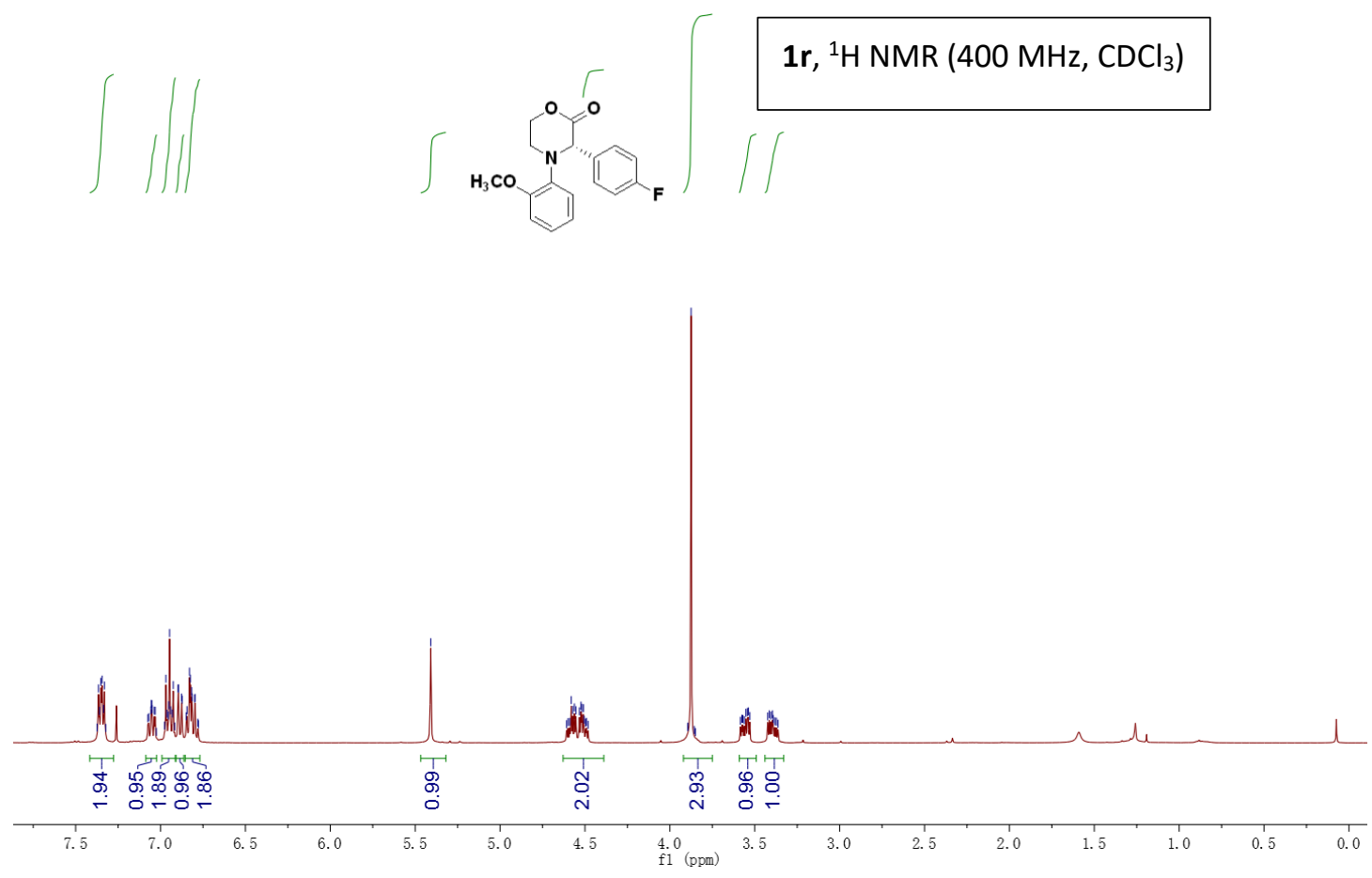

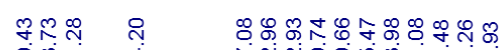

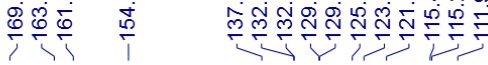

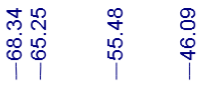

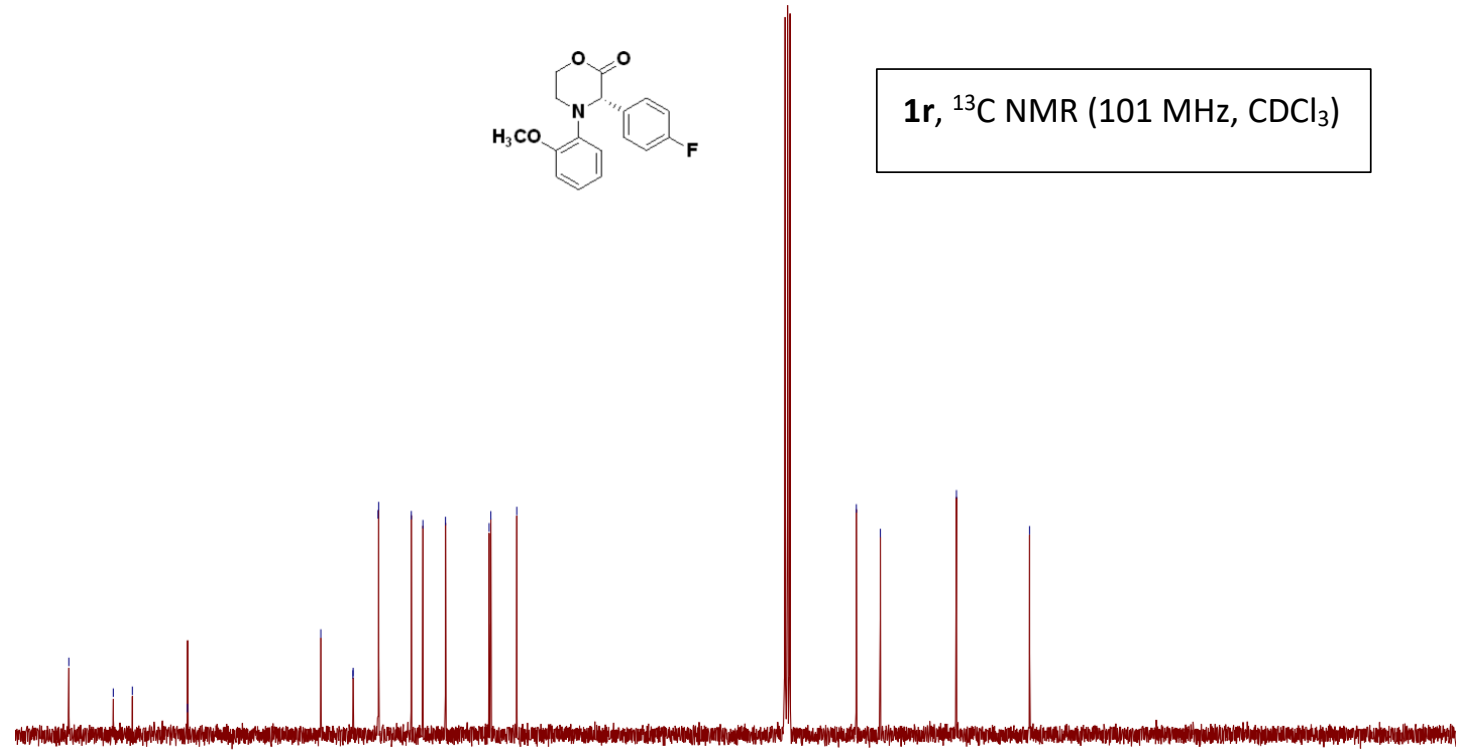

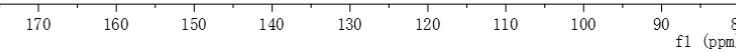



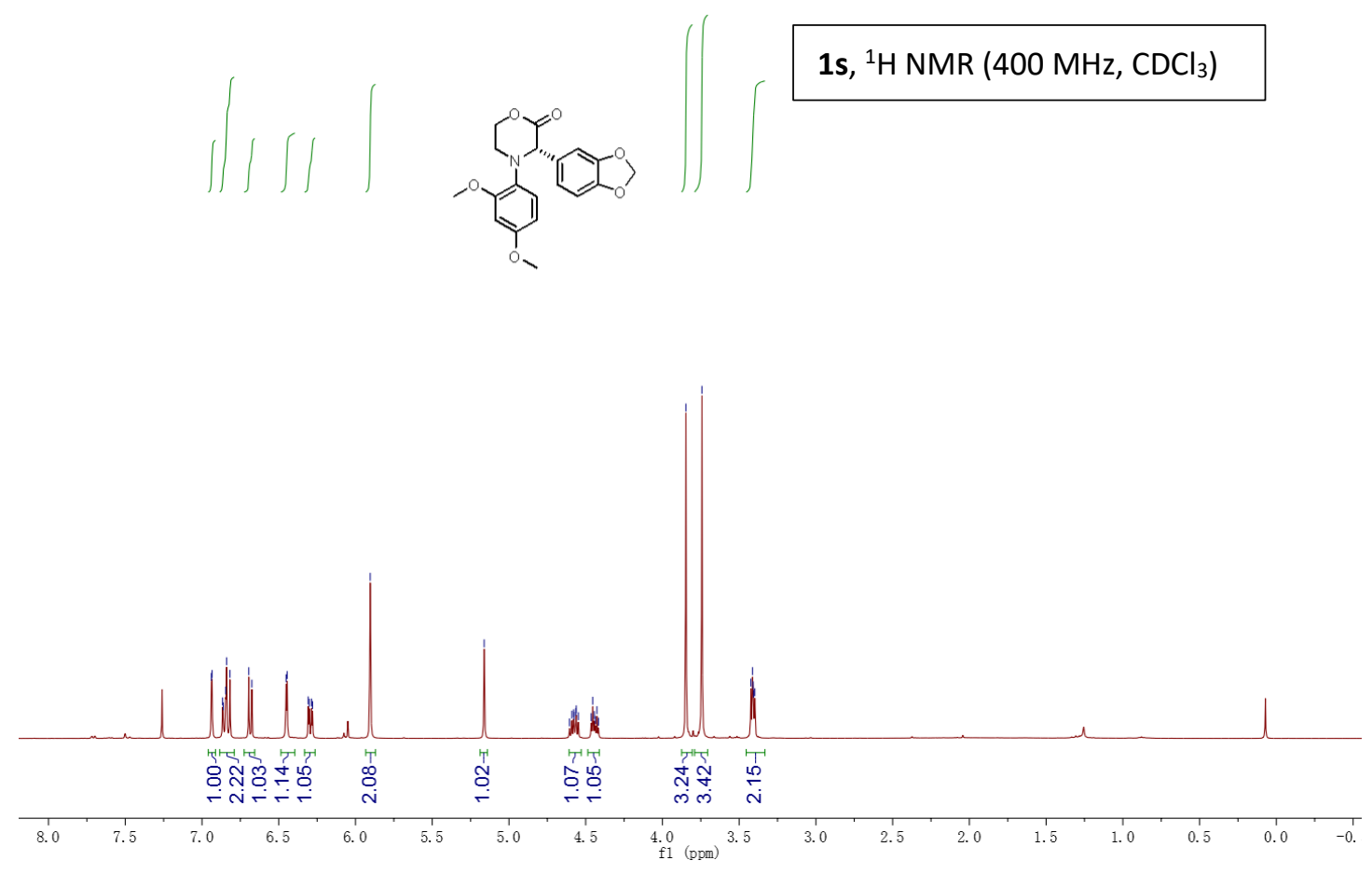

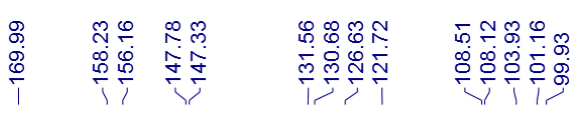

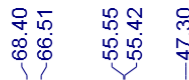

1s, ${ }^{13} \mathrm{C}$ NMR (101 MHz, $\left.\mathrm{CDCl}_{3}\right)$

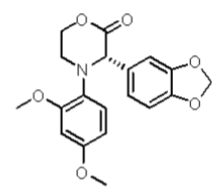

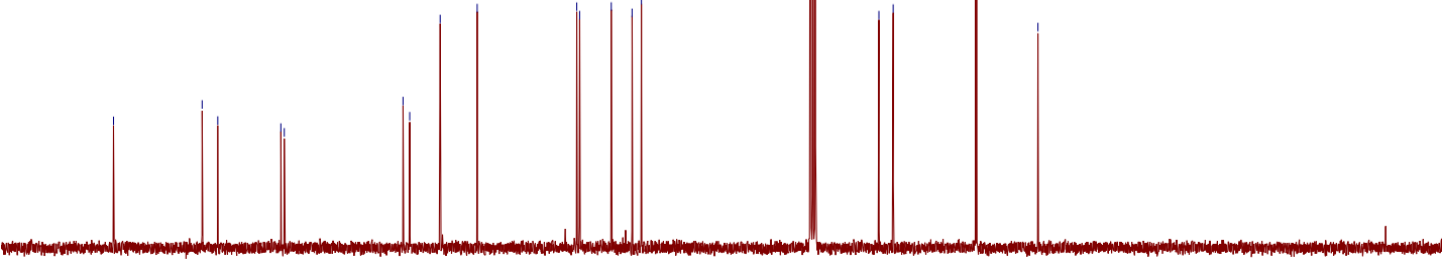

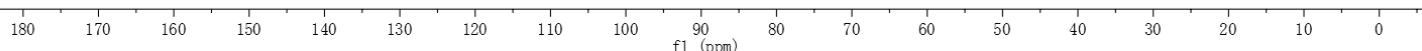




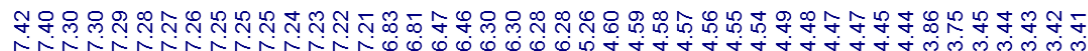

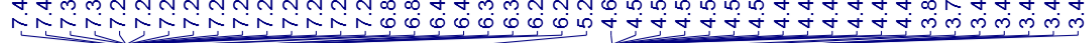
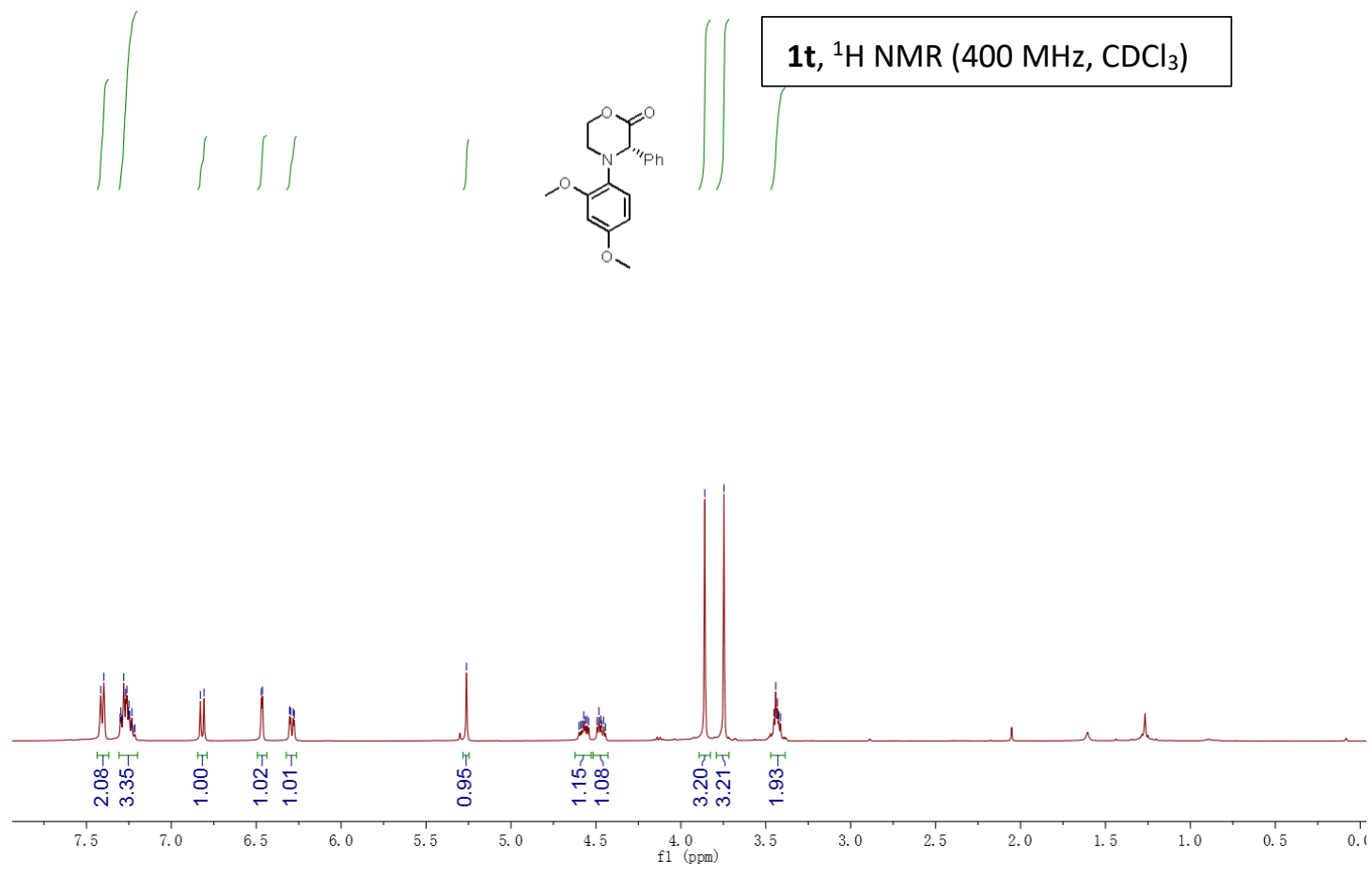

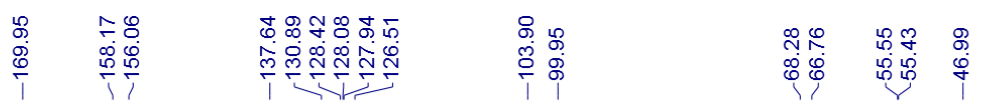

1t, ${ }^{13} \mathrm{C} \quad \mathrm{NMR} \quad(101 \mathrm{MHz}$

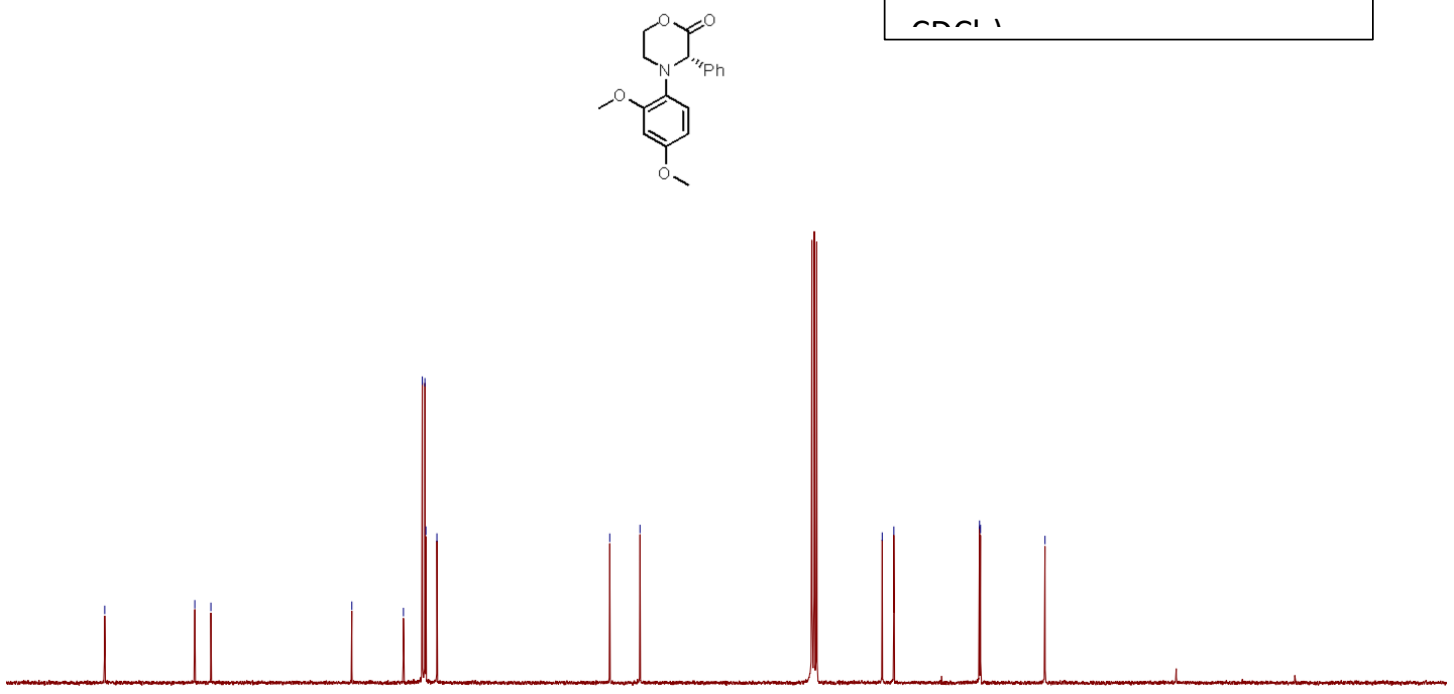

and

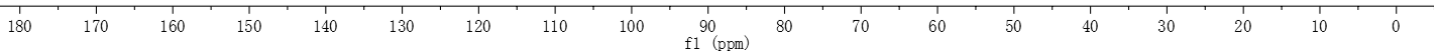




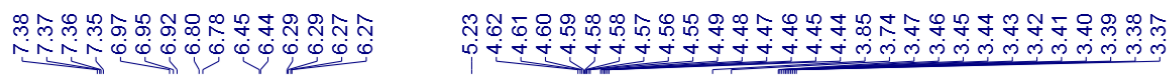
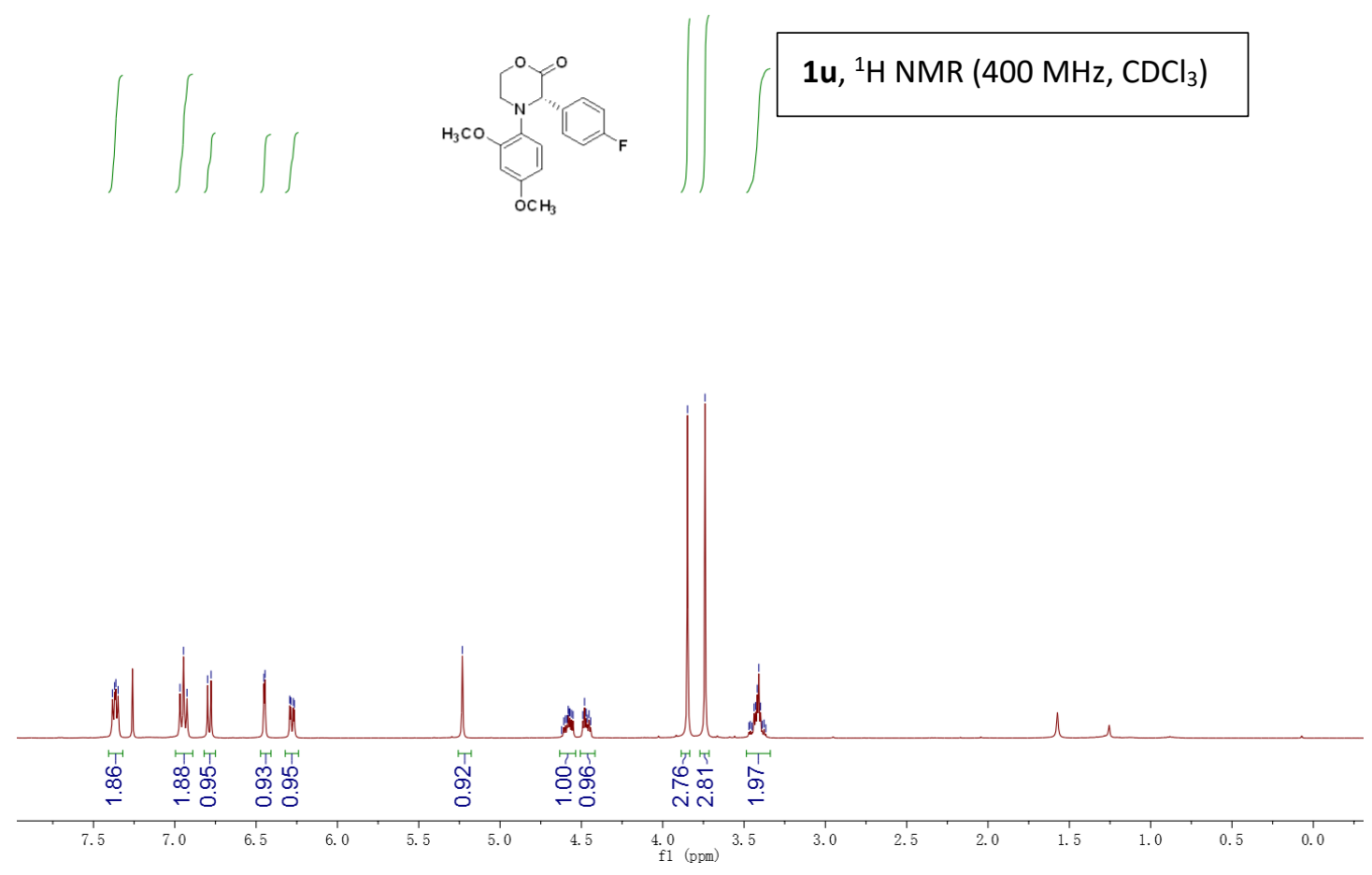

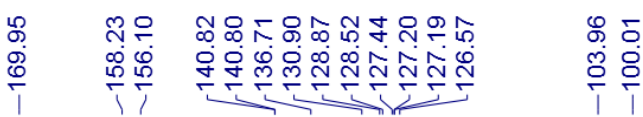

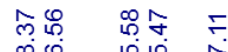

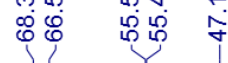

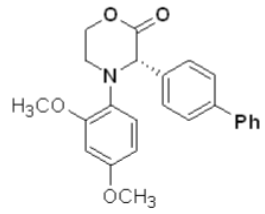

1u, ${ }^{13} \mathrm{C}$ NMR $\left(101 \mathrm{MHz}, \mathrm{CDCl}_{3}\right)$

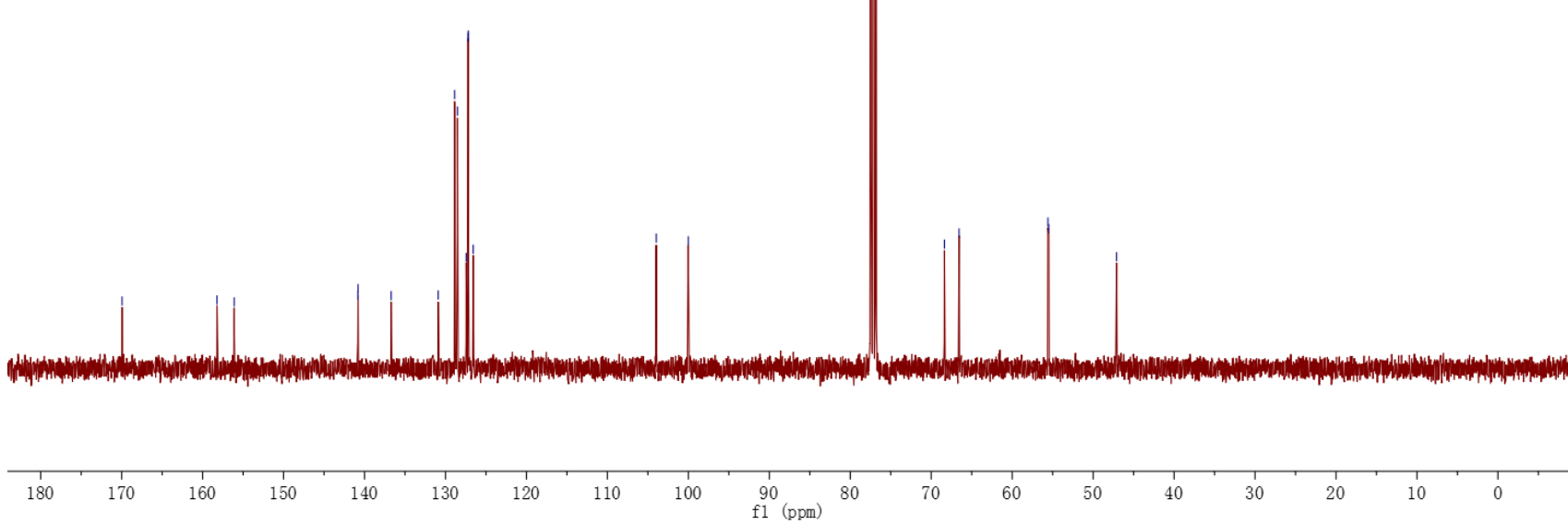




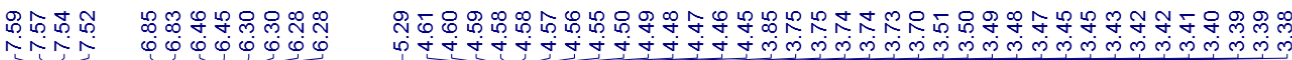
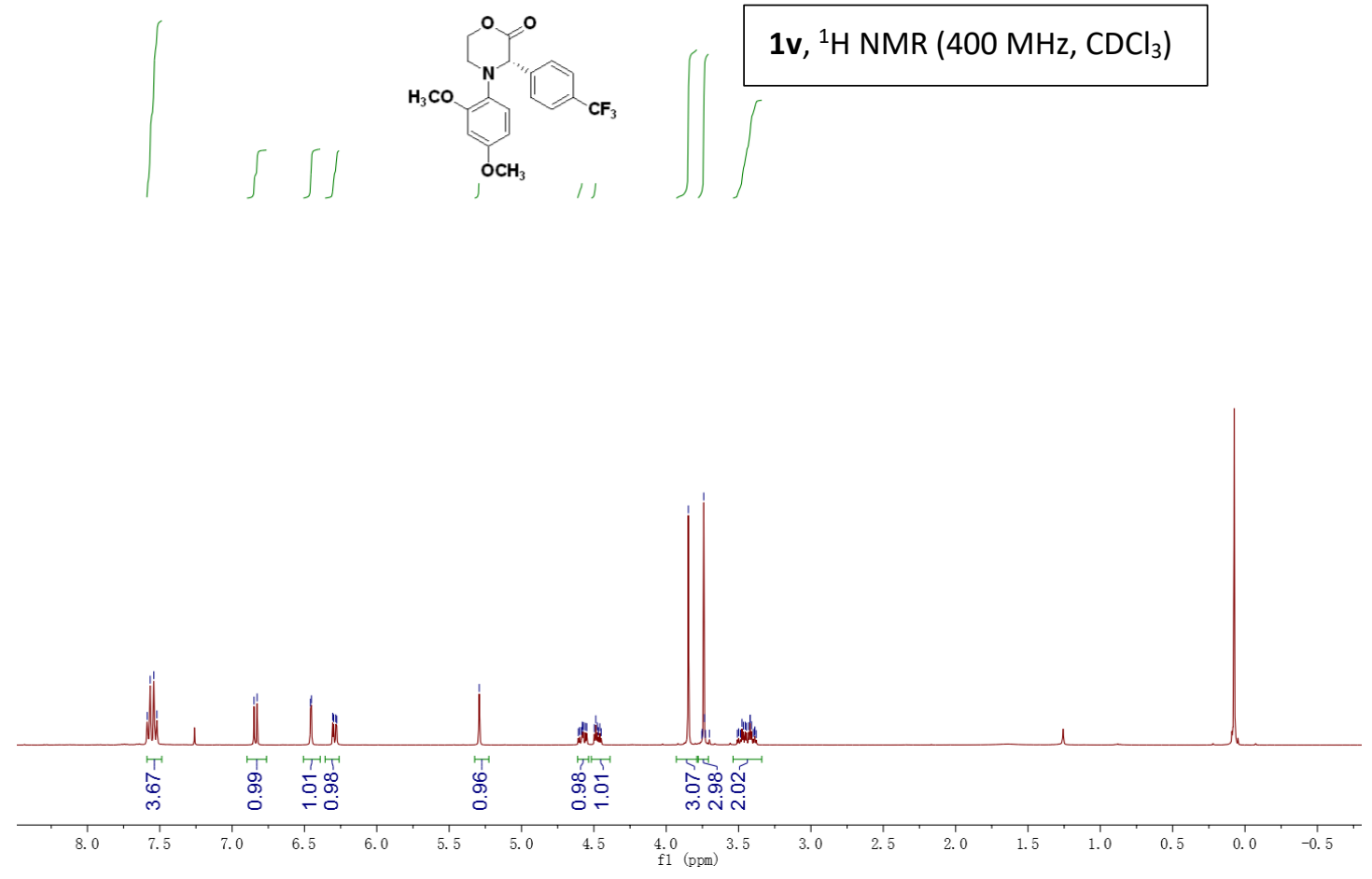

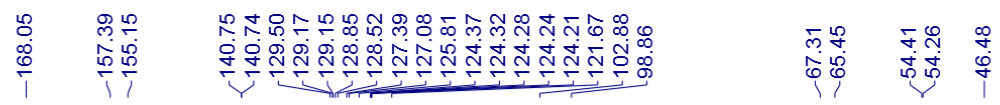
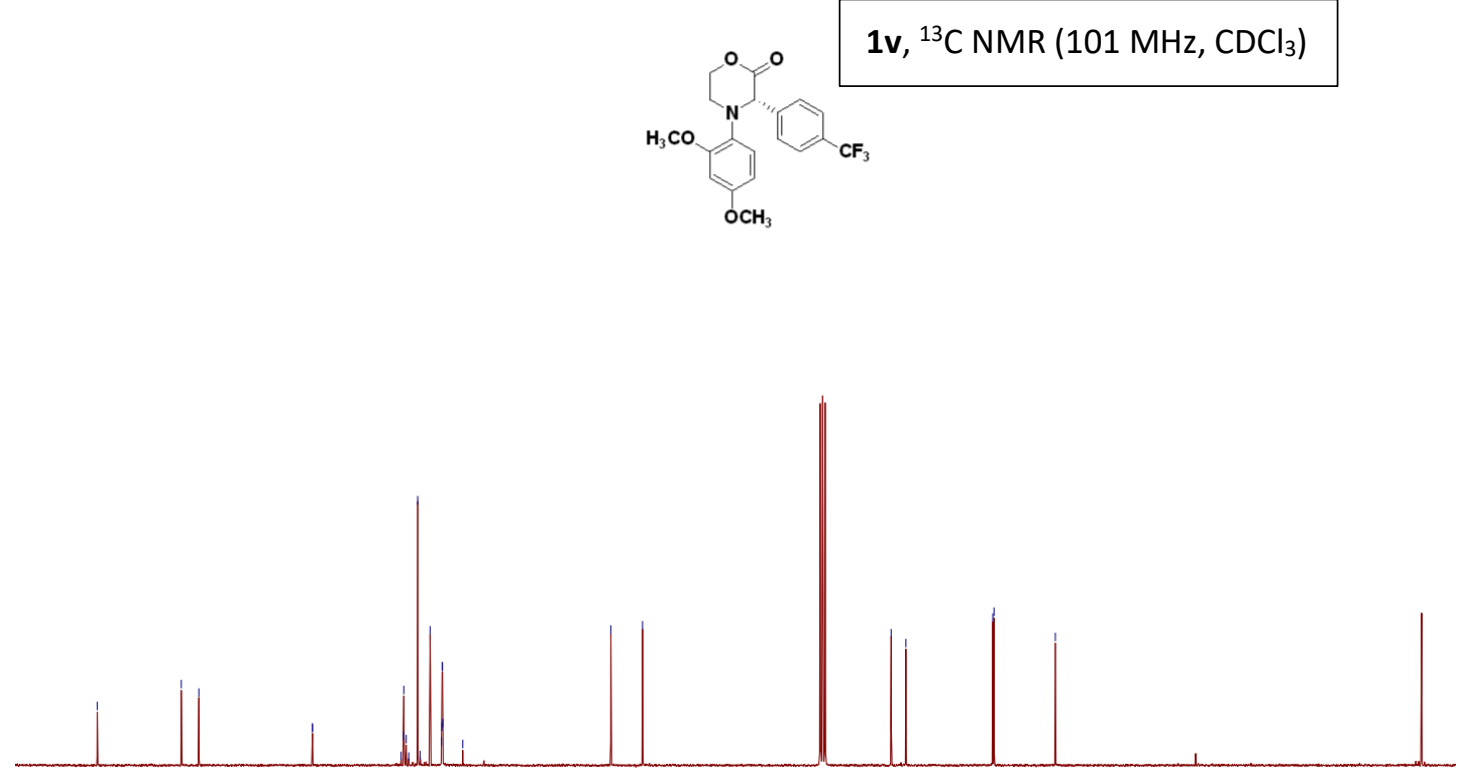

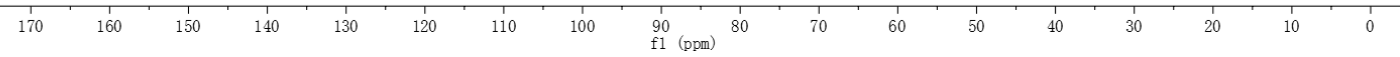



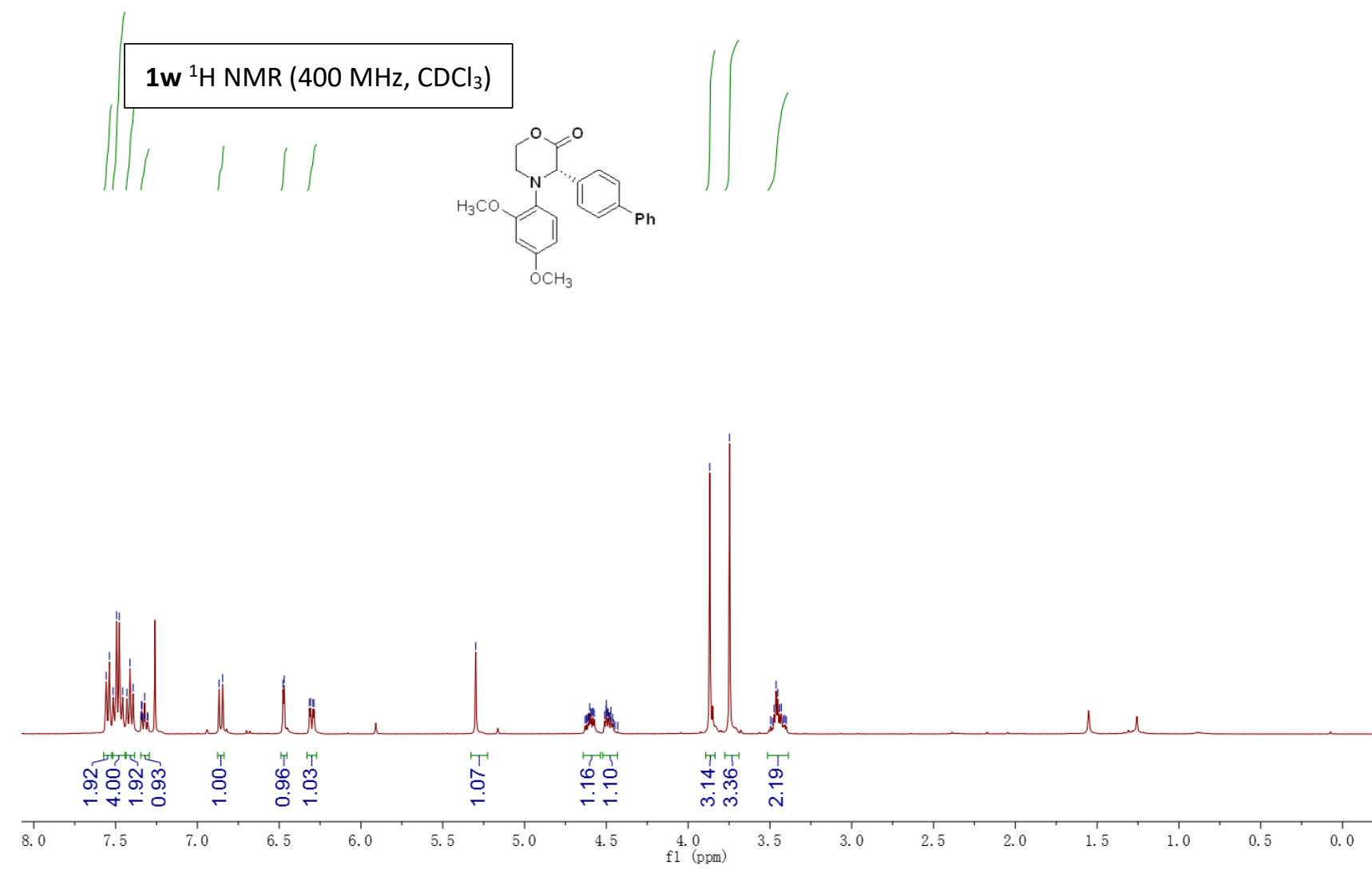

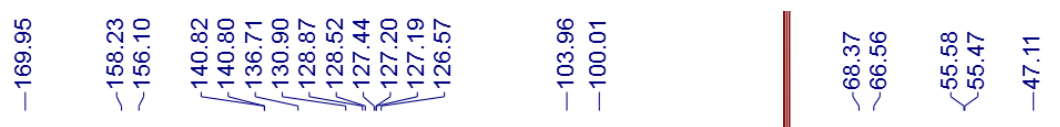

1w, ${ }^{13} \mathrm{C} \mathrm{NMR}\left(101 \mathrm{MHz}, \mathrm{CDCl}_{3}\right)$

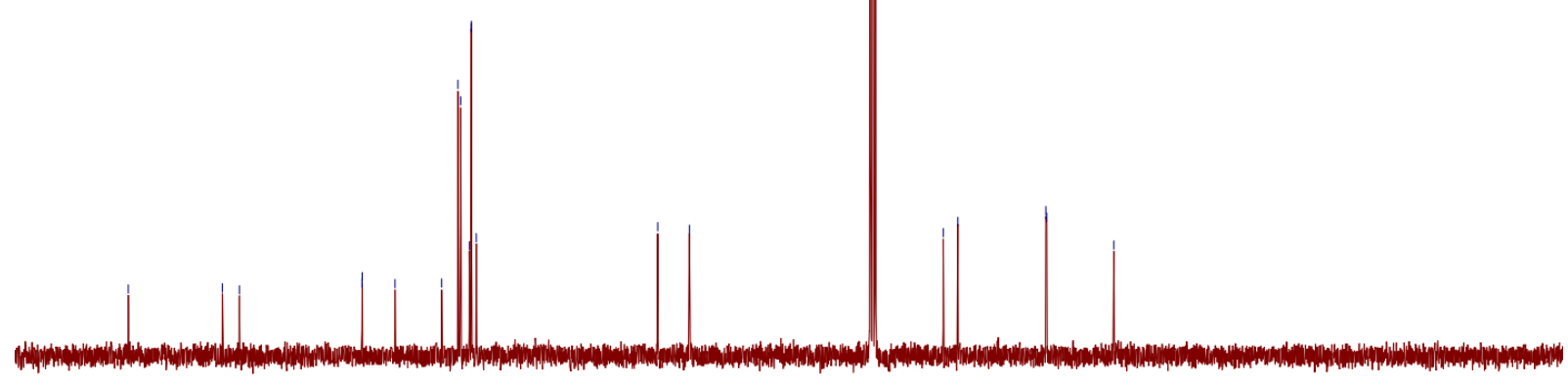

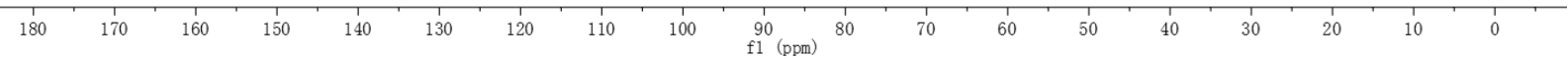




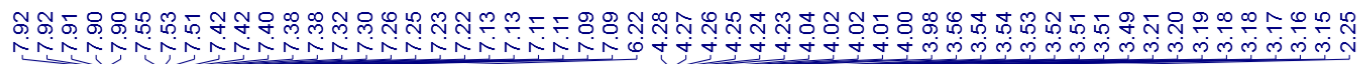

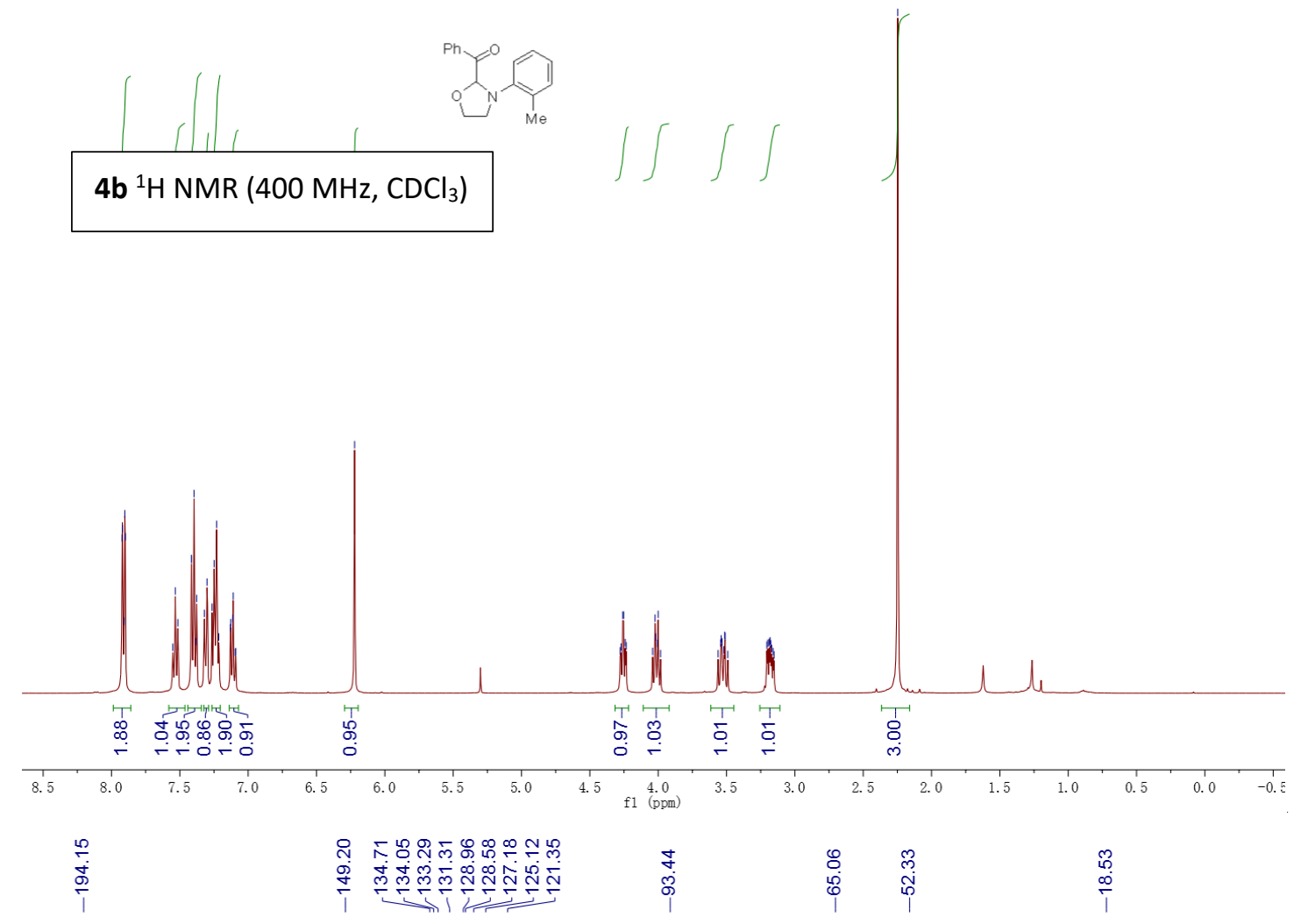

4b, ${ }^{13} \mathrm{C}$ NMR $(101 \mathrm{MHz}$,

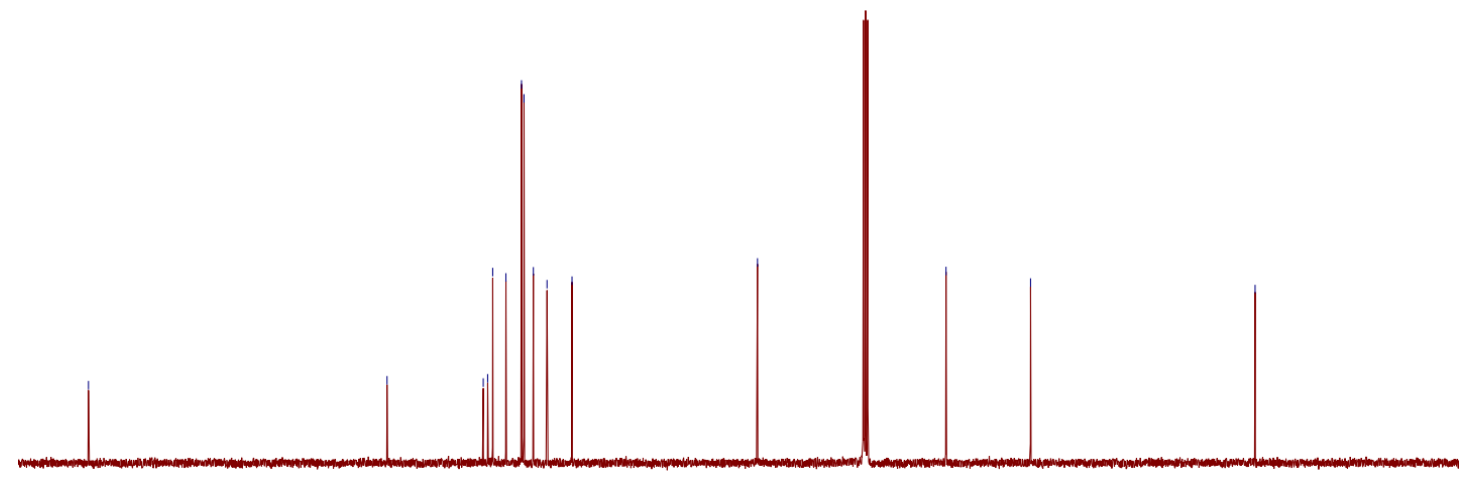

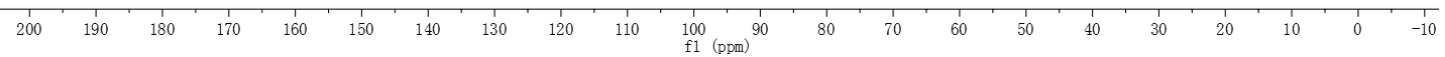




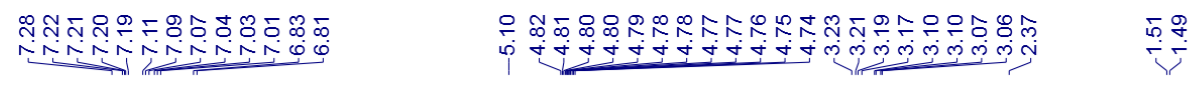

1x, ${ }^{1} \mathrm{H}$ NMR (400 MHz, $\mathrm{CDCl}_{3}$ )

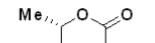

$\mathrm{Me}$

n",

$\checkmark$

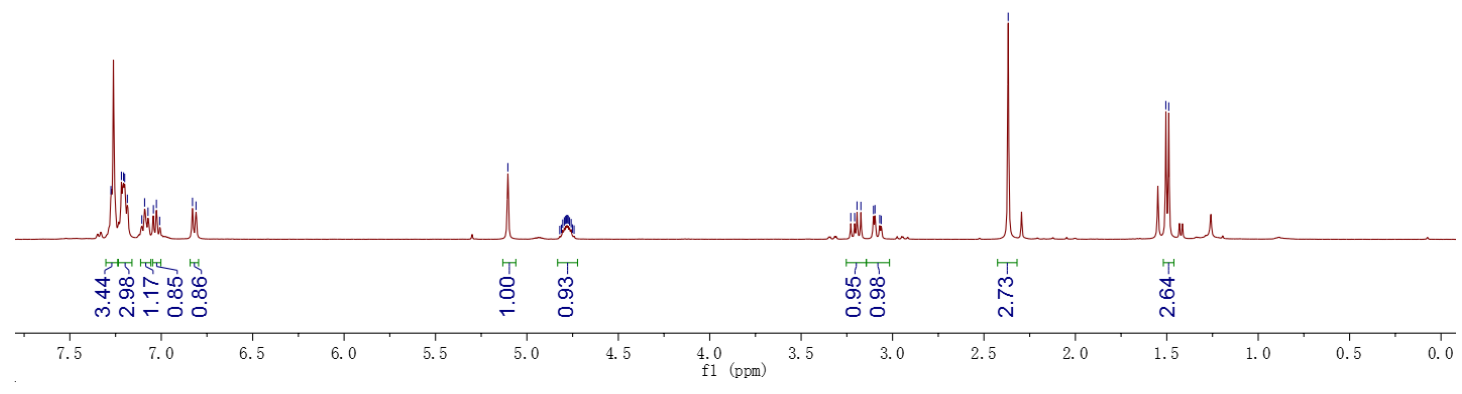

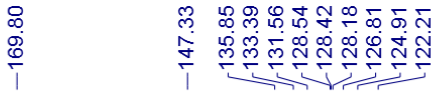

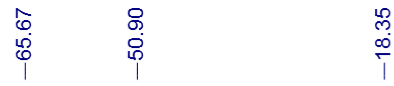

1x, ${ }^{13} \mathrm{C}$ NMR $\left(101 \mathrm{MHz}, \mathrm{CDCl}_{3}\right)$

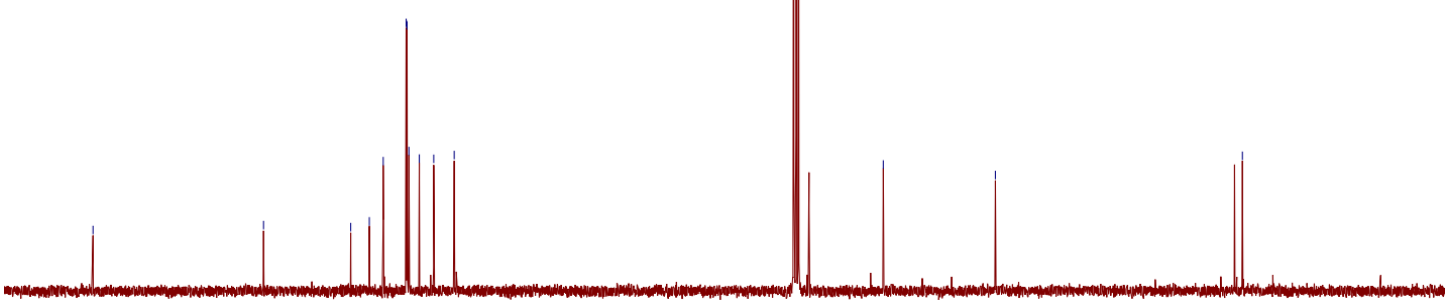

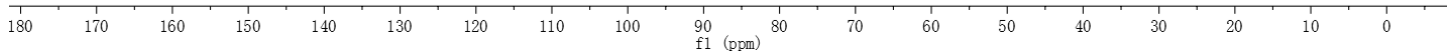




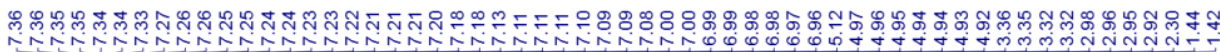

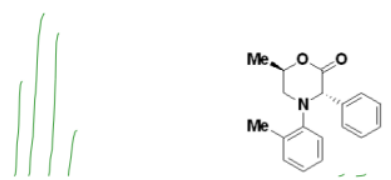

1y, ${ }^{1} \mathrm{H}$ NMR $\left(400 \mathrm{MHz}, \mathrm{CDCl}_{3}\right)$
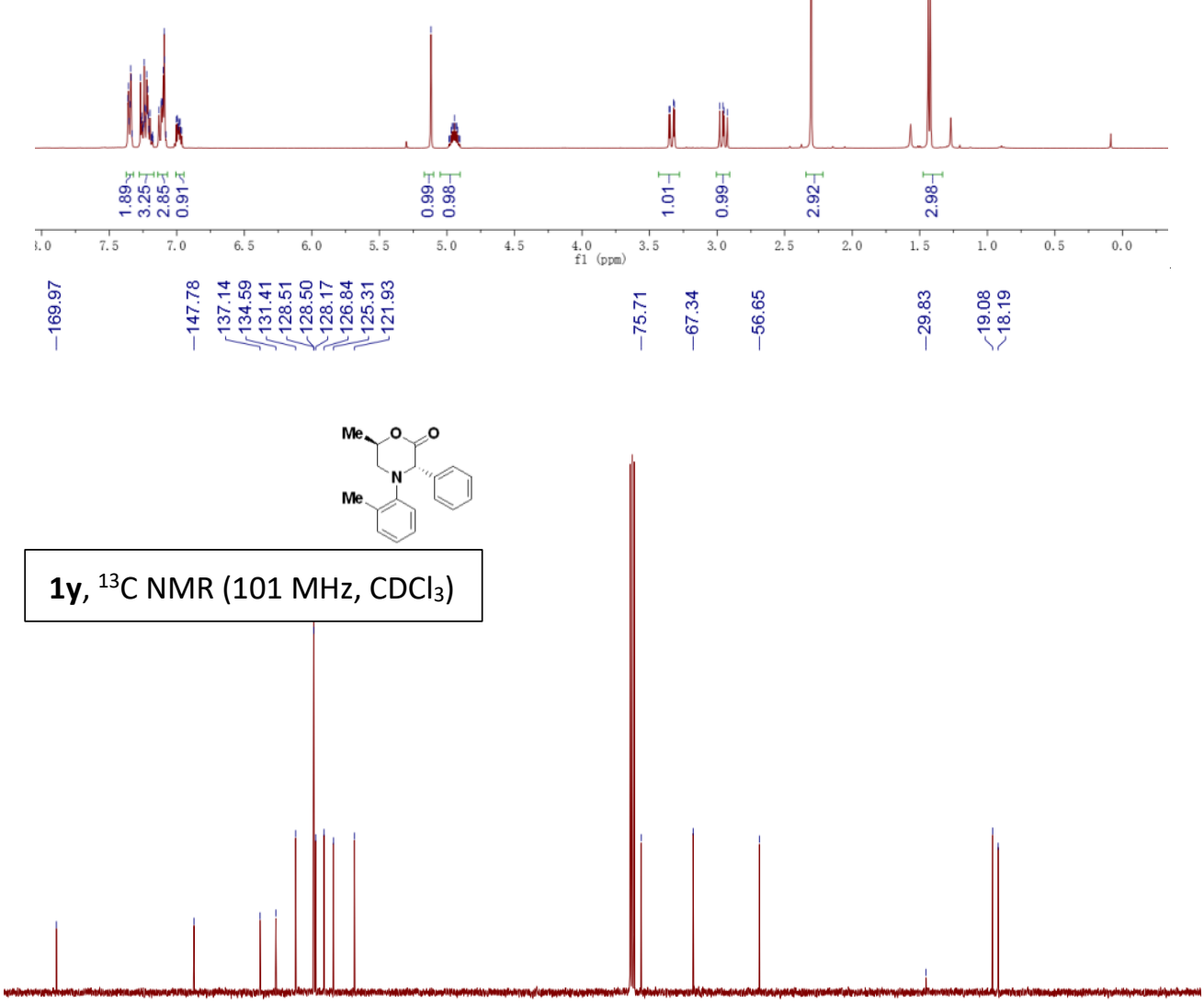

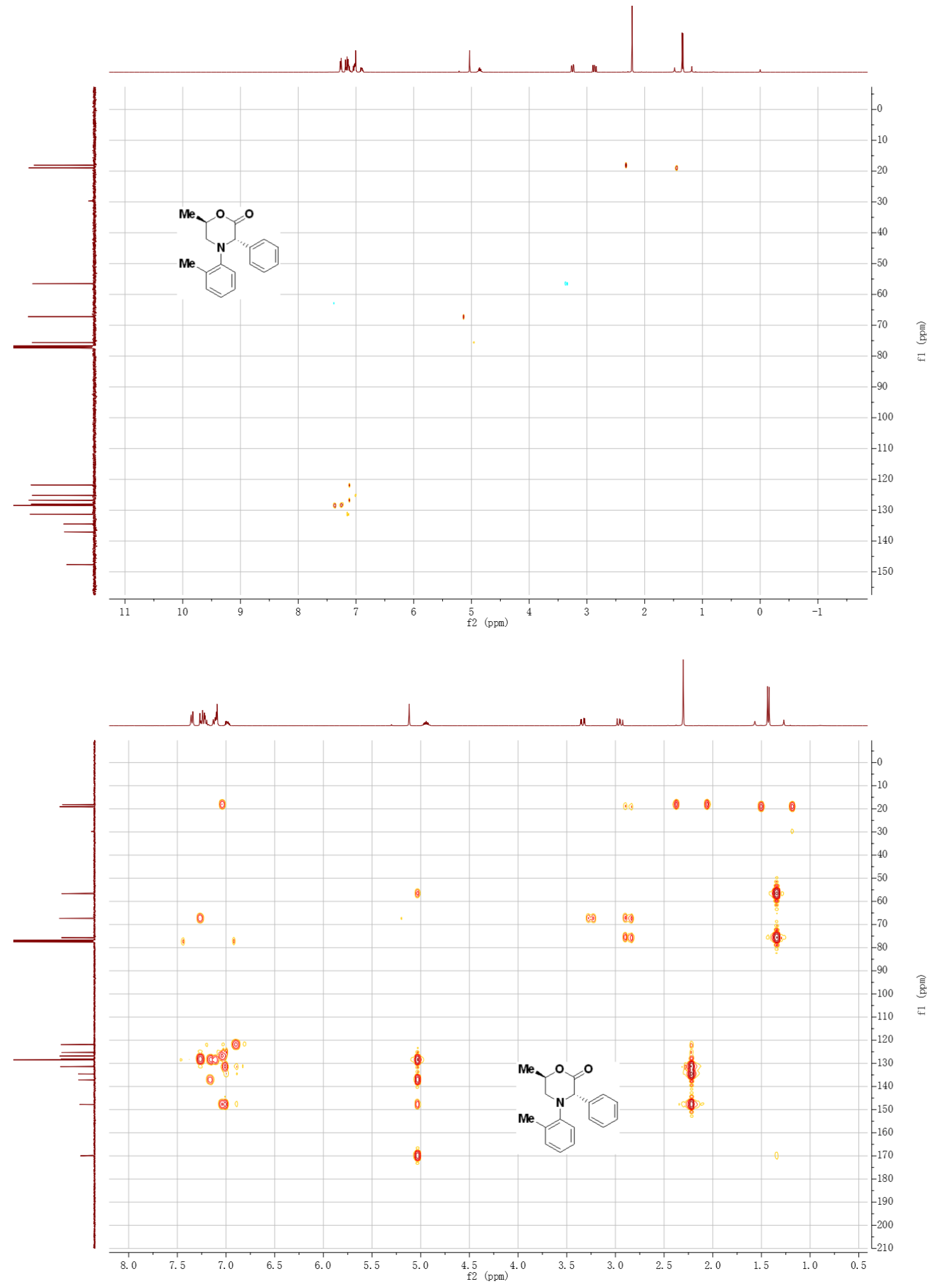


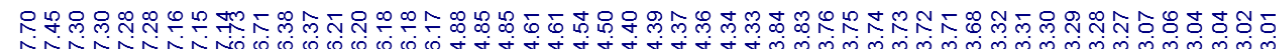

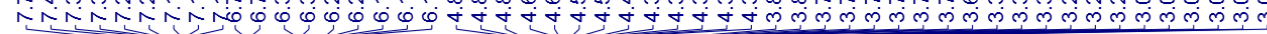

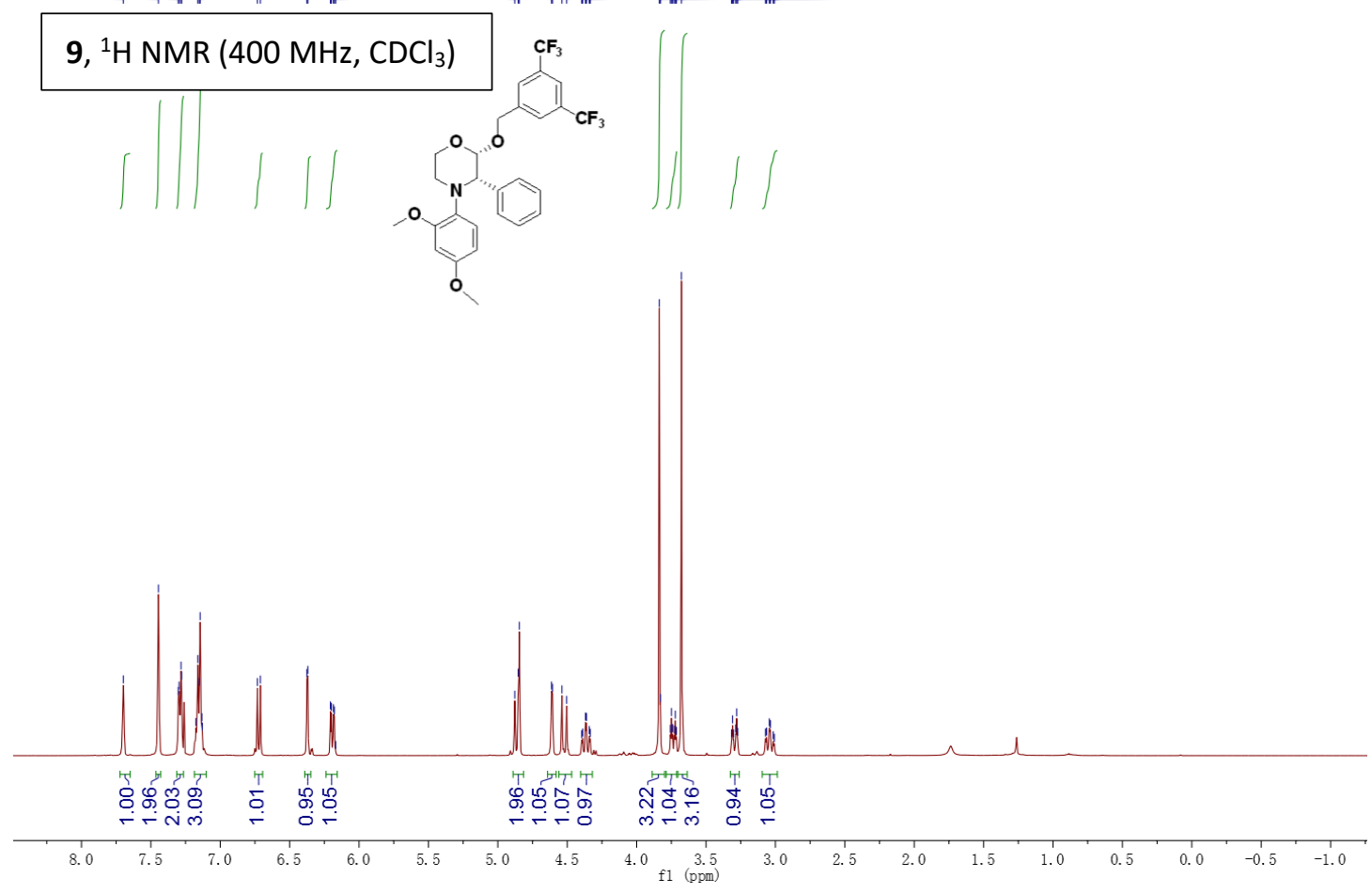

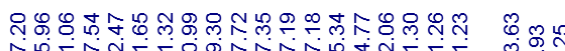

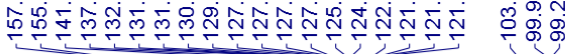

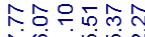

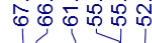

9, ${ }^{13} \mathrm{C}$ NMR $\left(101 \mathrm{MHz}, \mathrm{CDCl}_{3}\right)$
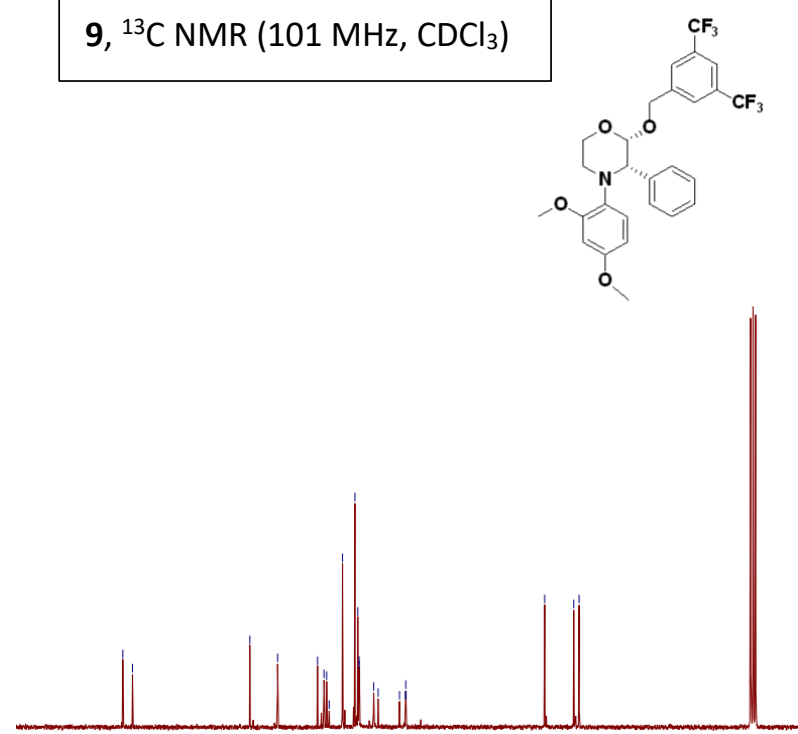

160
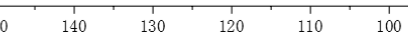

$90 \quad \frac{80}{\mathrm{f} 1}(\mathrm{ppm})$ 


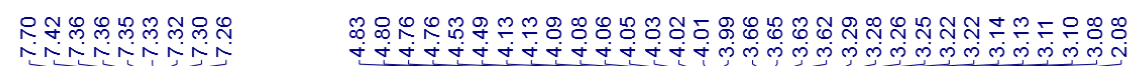
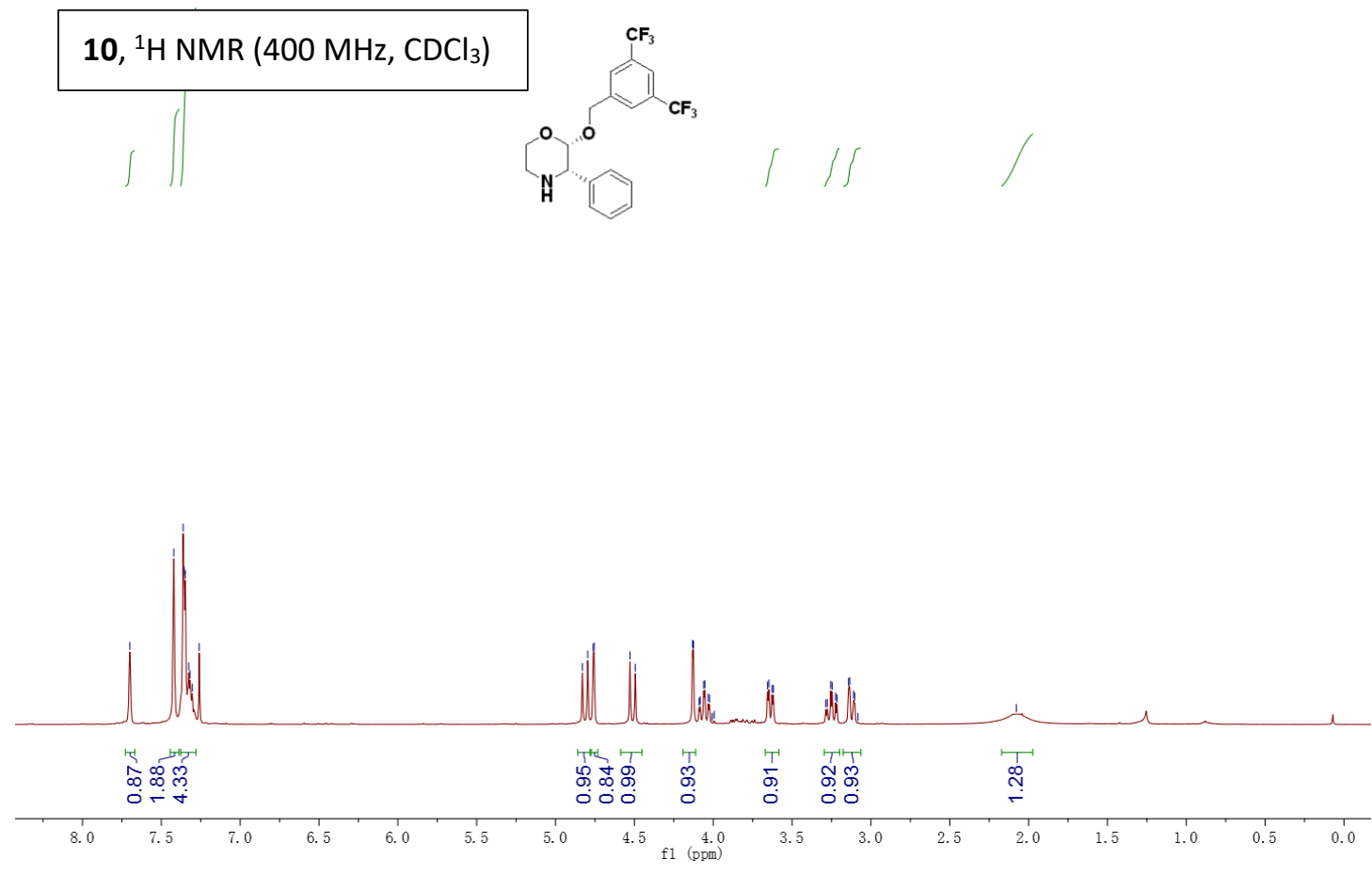

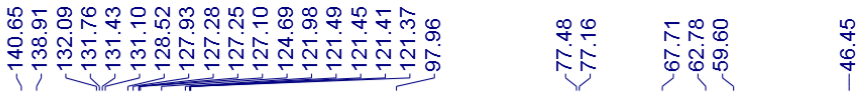

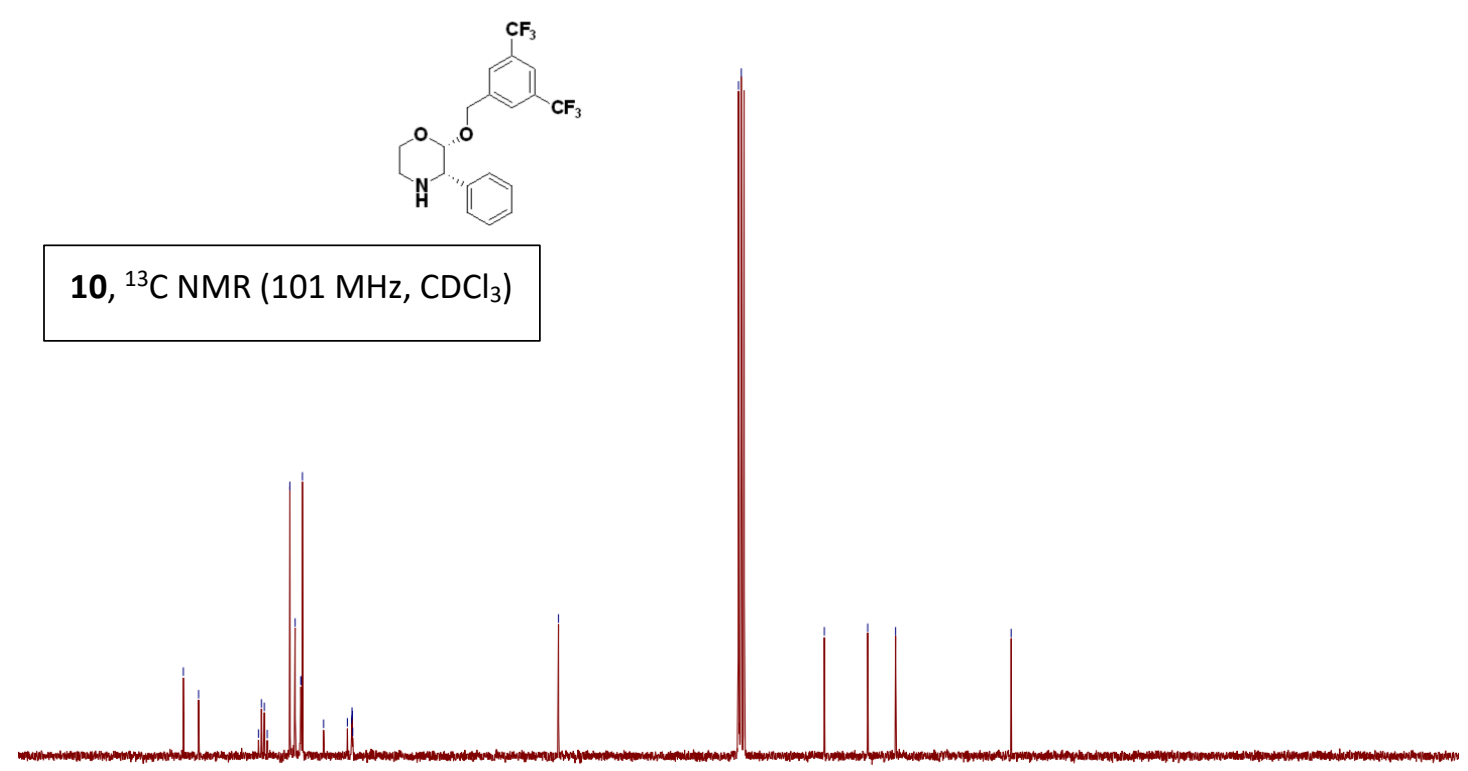

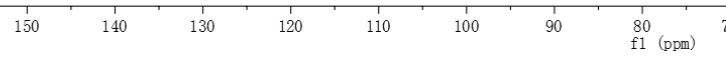




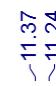

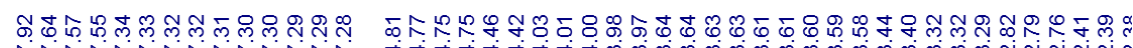<smiles></smiles>

\section{7, ${ }^{1} \mathrm{H}$ NMR (400 MHz, DMSO-d6)}
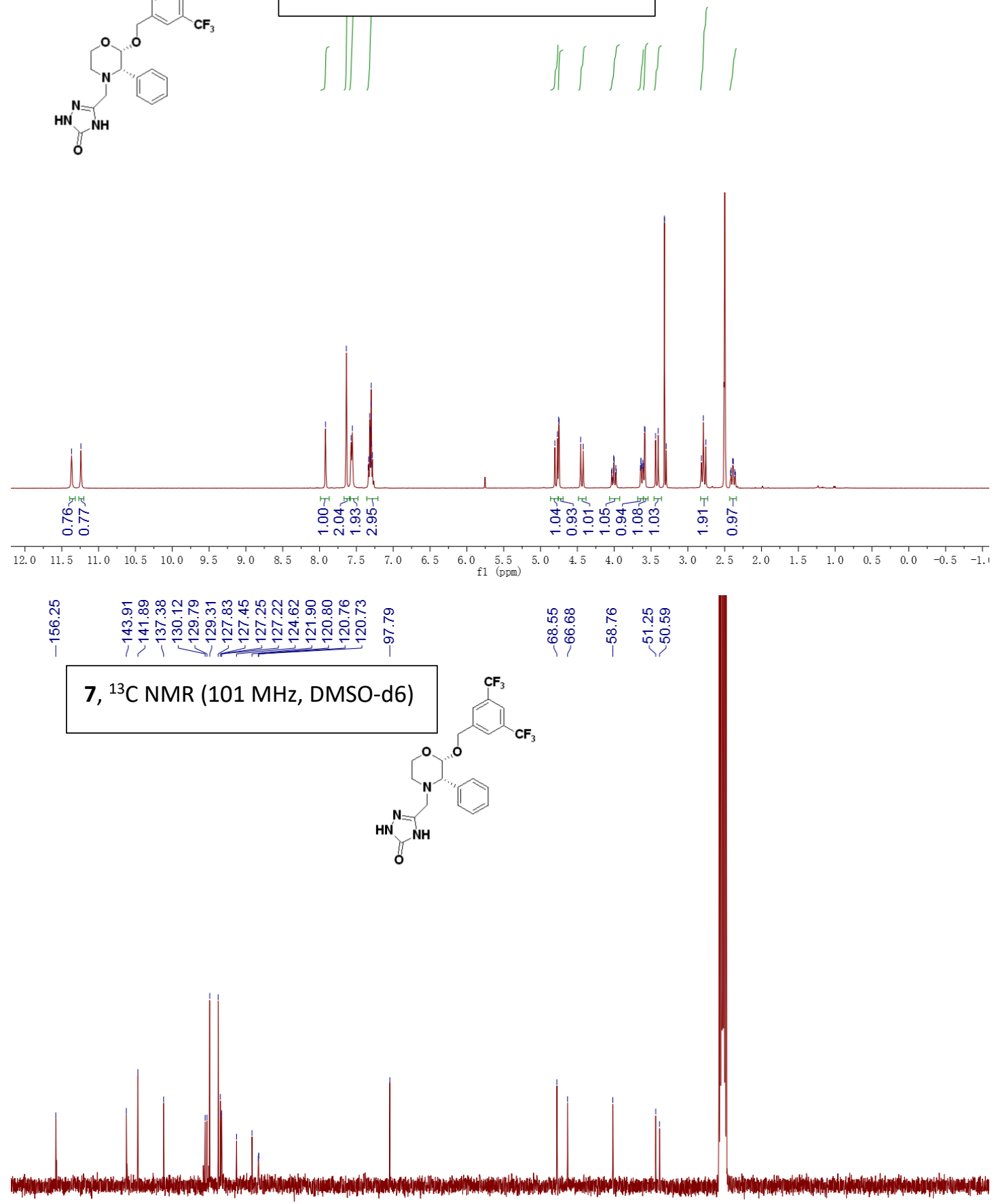

160

140

$130 \quad 120$

100

$\mathrm{fl}^{80}(\mathrm{ppm})$

$20,10+1$, 

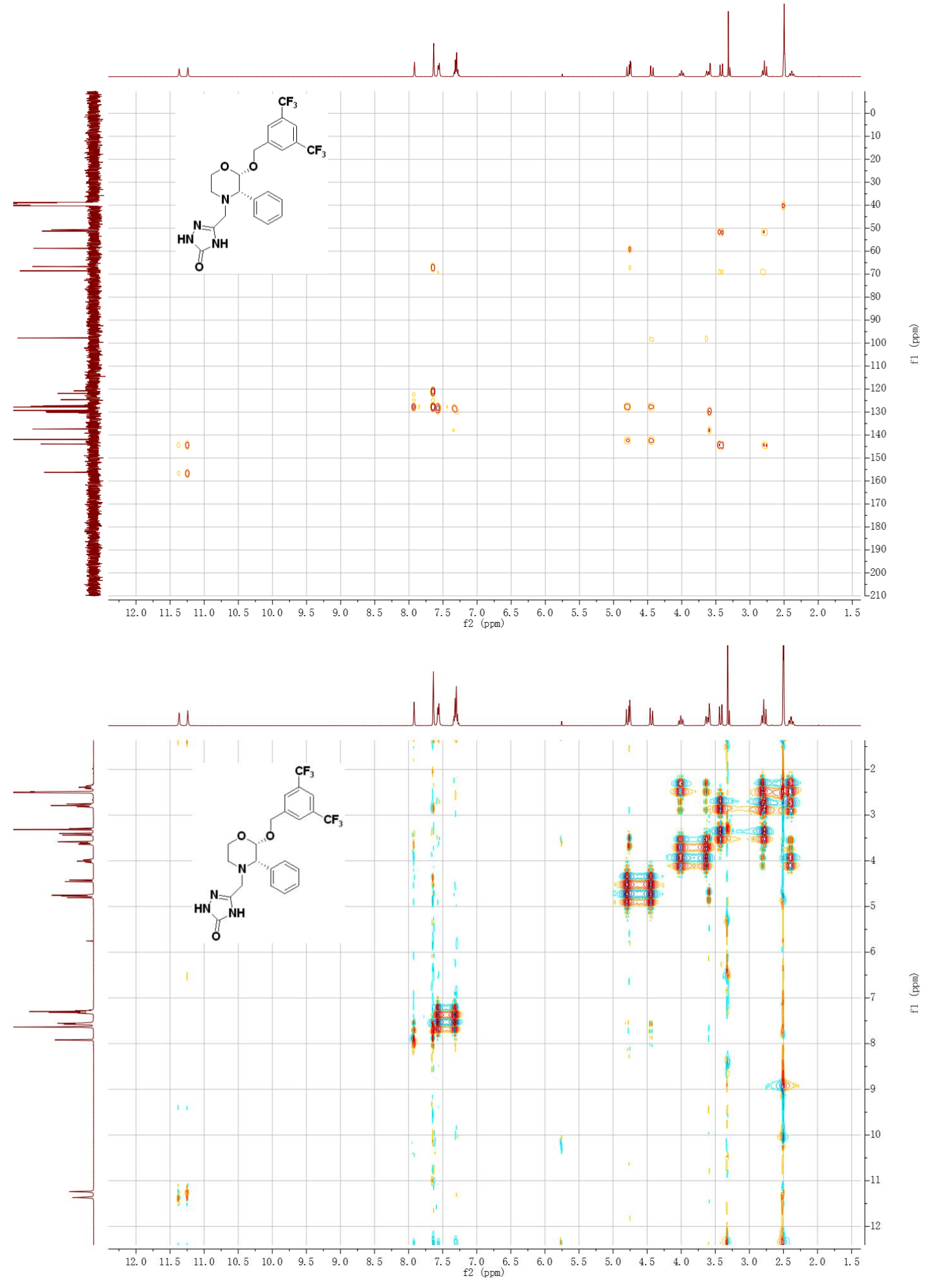
ஸి

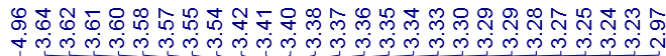

rivi

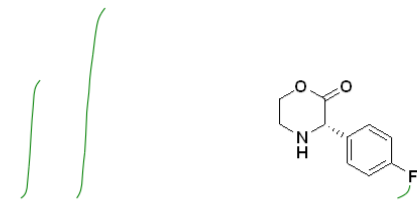

12, ${ }^{1} \mathrm{H}$ NMR $\left(400 \mathrm{MHz}, \mathrm{CDCl}_{3}\right)$

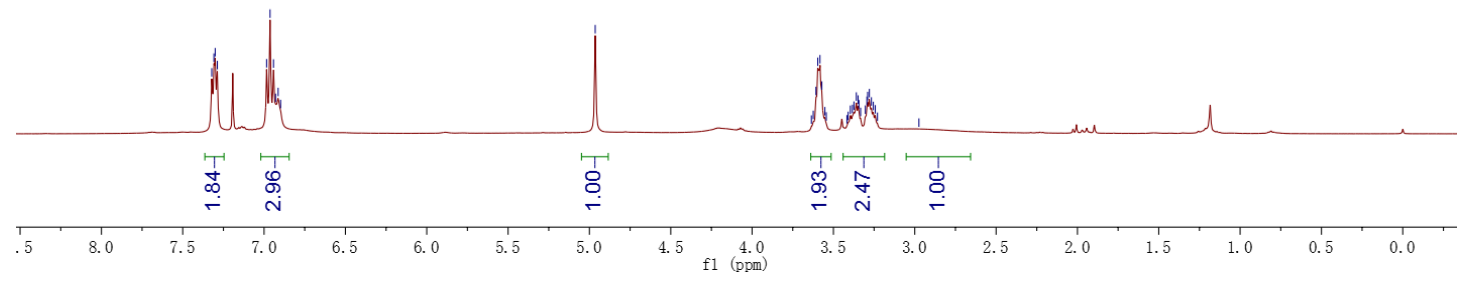

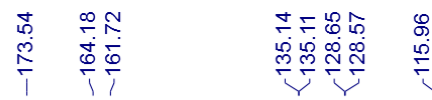

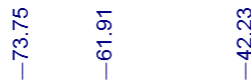

12, ${ }^{13} \mathrm{C}$ NMR $\left(101 \mathrm{MHz}, \mathrm{CDCl}_{3}\right)$

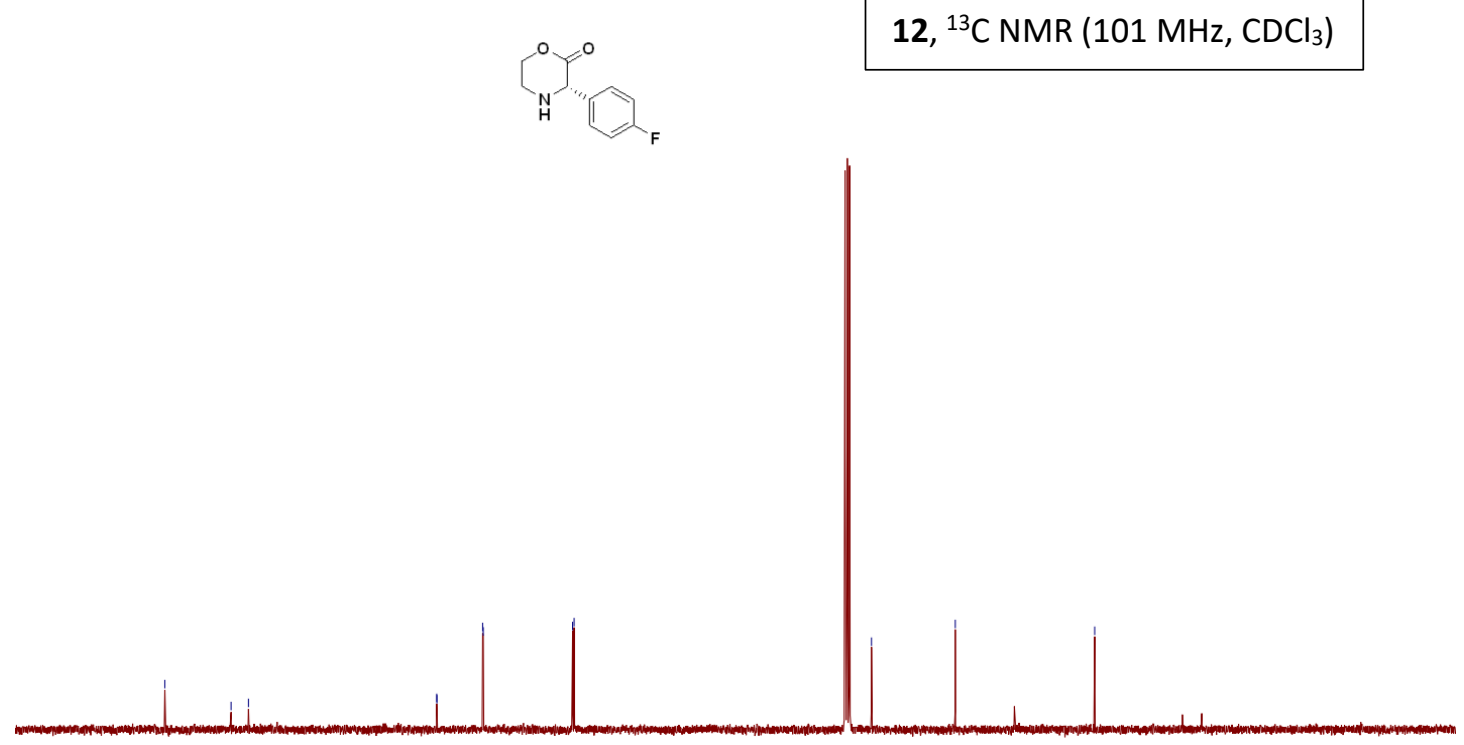

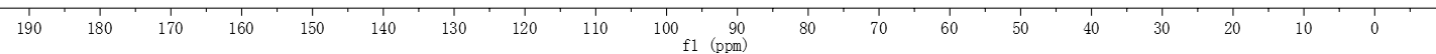




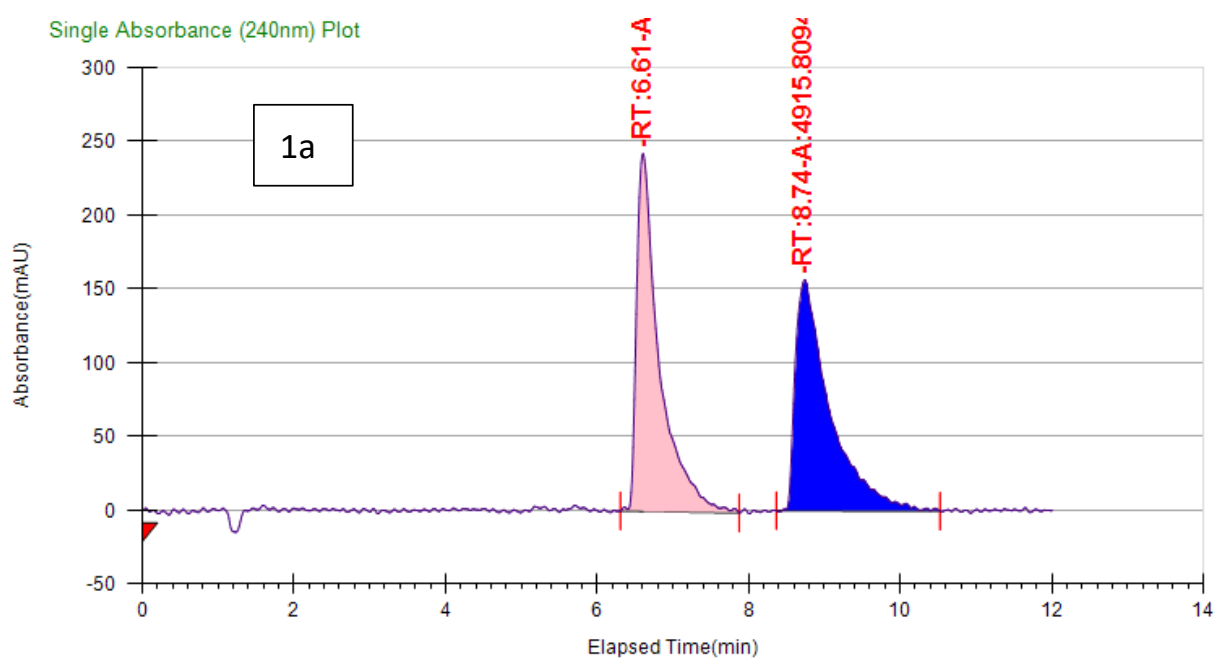

Run Information

\begin{tabular}{|l|l|l|l|l|l|l|l|l|l|}
\hline Instrument Method & Inj. Vol. & Solvent & Column & Sample & Well Location & Temp. & Flow & $\%$ Modifier & Pressure \\
\hline $5 p$ methanol & 10 & MeOH & IA & $\begin{array}{l}6-25-\text {-model- } \\
\text { A } 5 \% \%\end{array}$ & $11 \mathrm{~F}$ & 30.9 & 4 & 5 & 150 \\
\hline
\end{tabular}

Peak Information

\begin{tabular}{|l|l|l|l|l|l|}
\hline Peak No & $\%$ Area & Area & Ret. Time & Height & Cap. Factor \\
\hline 1 & 50.6057 & 5036.3676 & $6.61 \mathrm{~min}$ & 242.5383 & 0 \\
\hline 2 & 49.3943 & 4915.8094 & $8.74 \mathrm{~min}$ & 156.7357 & 0 \\
\hline
\end{tabular}

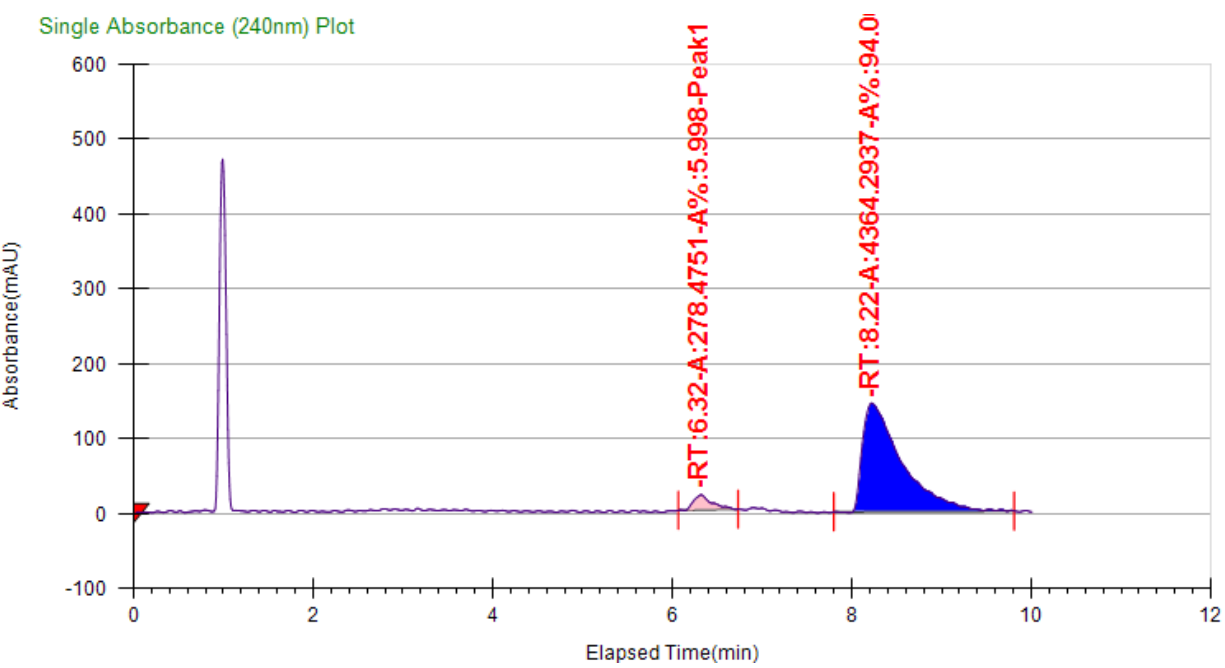

Run Information

\begin{tabular}{|l|l|l|l|l|l|l|l|l|l|}
\hline Instrument Method & Inj. Vol. & Solvent & Column & Sample & Well Location & Temp. & Flow & $\%$ Modifier & Pressure \\
\hline $5 p$ methanol & 10 & MeOH & IA & $\begin{array}{l}\text { yp-hwu-6- } \\
25 \text { 'old- } \\
\text { trimetyllPAl- } \\
\text { IA5\% }\end{array}$ & $11 \mathrm{D}$ & 30 & 4 & 5 & 150 \\
\hline
\end{tabular}

Peak Information

\begin{tabular}{|l|l|l|l|l|l|}
\hline Peak No & $\%$ Area & Area & Ret. Time & Height & Cap. Factor \\
\hline 1 & 5.998 & 278.4751 & $6.32 \mathrm{~min}$ & 20.1077 & 6323.9167 \\
\hline 2 & 94.002 & 4364.2937 & $8.22 \mathrm{~min}$ & 145.0408 & 8223.8833 \\
\hline
\end{tabular}




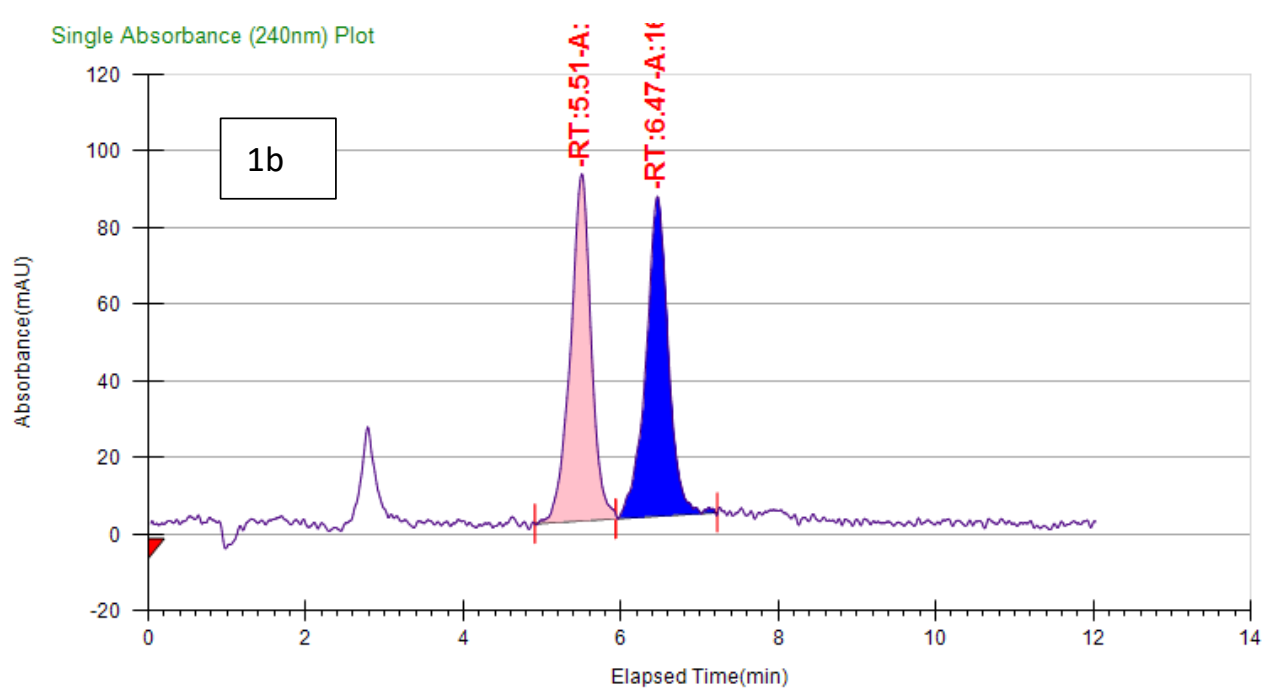

Run Information

\begin{tabular}{|l|l|l|l|l|l|l|l|l|l|}
\hline Instrument Method & Inj. Vol. & Solvent & Column & Sample & Well Location & Temp. & Flow & $\%$ Modifier & Pressure \\
\hline $5 p$ methanol & 10 & MeOH & AD-H & $\begin{array}{l}\text { yp-hwu-6-26- } \\
2 \text { Me'---LL- } \\
\text { AD5\% }\end{array}$ & $11 \mathrm{~F}$ & 30.1 & 4 & 5 & 150 \\
\hline
\end{tabular}

Peak Information

\begin{tabular}{|l|l|l|l|l|l|}
\hline Peak No & $\%$ Area & Area & Ret. Time & Height & Cap. Factor \\
\hline 1 & 50.0255 & 1651.5302 & $5.51 \mathrm{~min}$ & 90.5512 & 5507.25 \\
\hline 2 & 49.9745 & 1649.8449 & $6.47 \mathrm{~min}$ & 83.5145 & 6465.5833 \\
\hline
\end{tabular}

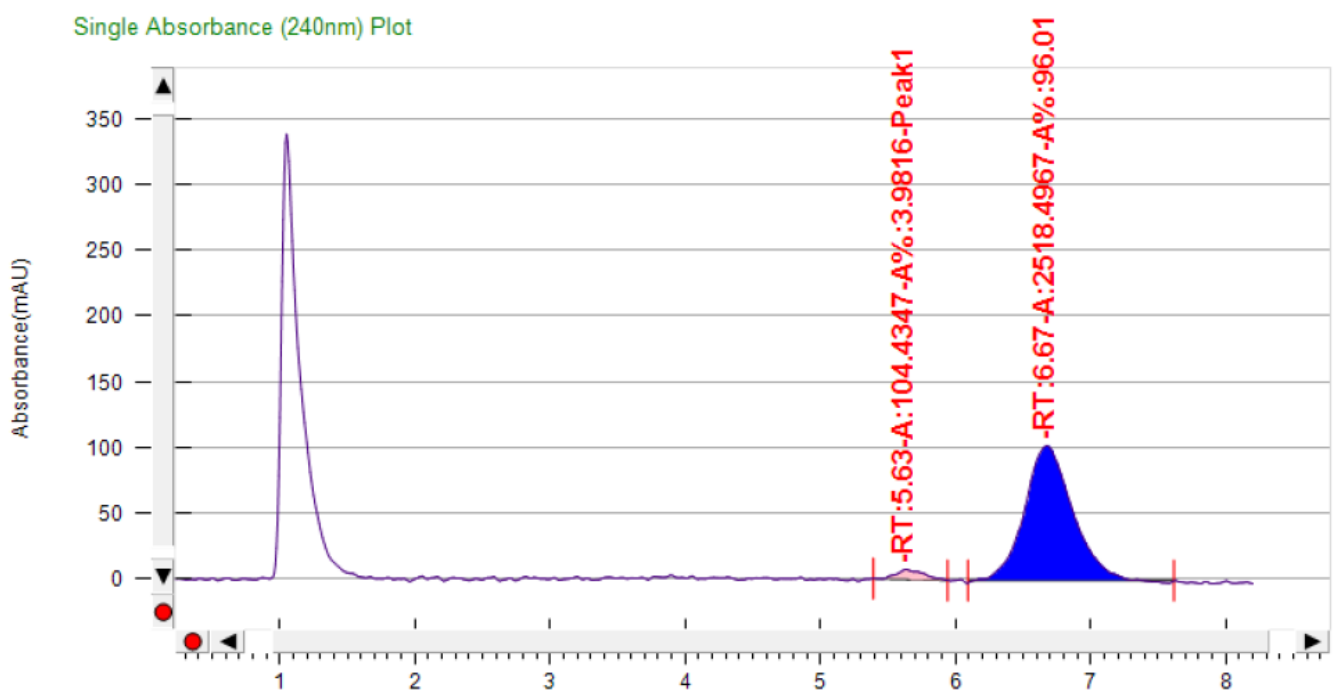

Run Information

\begin{tabular}{|l|l|l|l|l|l|l|l|l|l|}
\hline Instrument Method & Inj. Vol. & Solvent & Column & Sample & Well Location & Temp. & Flow & $\%$ Modifier & Pressure \\
\hline $5 p$ methanol & 10 & MeOH & AD-H & $\begin{array}{l}\text { yp-hwu-6-26- } \\
\text { 2Me-Re- } \\
700 C-a s y- \\
\text { AD510\% }\end{array}$ & $11 \mathrm{~B}$ & 31.2 & 4 & 5 & 150 \\
\hline
\end{tabular}

Peak Information

\begin{tabular}{|l|l|l|l|l|l|}
\hline Peak No & \% Area & Area & Ret. Time & Height & Cap. Factor \\
\hline 1 & 3.9816 & 104.4347 & $5.63 \mathrm{~min}$ & 7.6866 & 5632.25 \\
\hline 2 & 96.0184 & 2518.4967 & $6.67 \mathrm{~min}$ & 102.4312 & 6673.9 \\
\hline
\end{tabular}




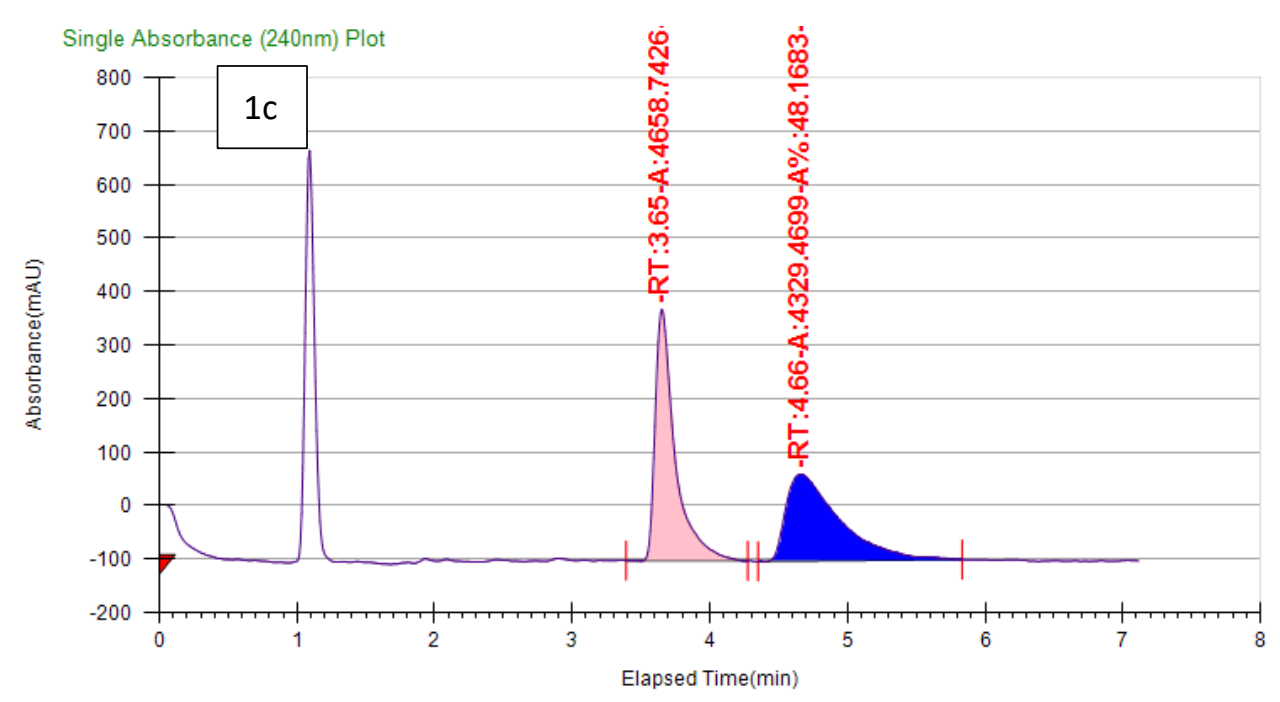

Run Information

\begin{tabular}{|l|l|l|l|l|l|l|l|l|l|}
\hline Instrument Method & Inj. Vol. & Solvent & Column & Sample & Well Location & Temp. & Flow & $\%$ Modifier & Pressure \\
\hline $30 p$ methanol & 10 & MeOH & IA & $\begin{array}{l}\text { yp-6- } \\
61^{\prime} \text { 'D'IA30\% }^{\prime}\end{array}$ & $11 \mathrm{~B}$ & 30 & 4 & 30 & 150 \\
\hline
\end{tabular}

Peak Information

\begin{tabular}{|l|l|l|l|l|l|}
\hline Peak No & \% Area & Area & Ret. Time & Height & Cap. Factor \\
\hline 1 & 51.8317 & 4658.7426 & $3.65 \mathrm{~min}$ & 469.4398 & 0 \\
\hline 2 & 48.1683 & 4329.4699 & $4.66 \mathrm{~min}$ & 162.2995 & 0 \\
\hline
\end{tabular}

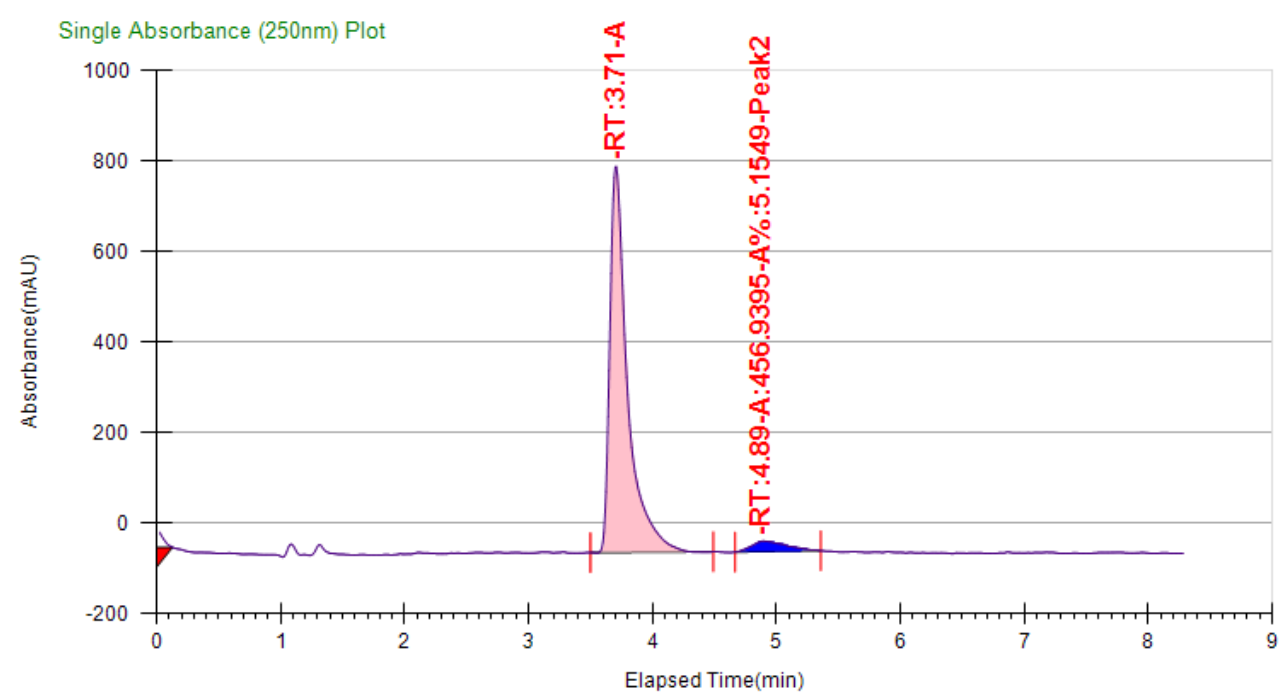

Run Information

\begin{tabular}{|l|l|l|l|l|l|l|l|l|l|}
\hline Instrument Method & Inj. Vol. & Solvent & Column & Sample & Well Location & Temp. & Flow & $\%$ Modifier & Pressure \\
\hline 30p methanol & 10 & $\mathrm{MeOH}$ & IA & $\begin{array}{l}\text { yp-6-61- } \\
700 \mathrm{C}^{\prime} \mid \mathrm{A} 30 \%\end{array}$ & $11 \mathrm{E}$ & 30 & 4 & 30 & 150 \\
\hline
\end{tabular}

Peak Information

\begin{tabular}{|l|l|l|l|l|l|}
\hline Peak No & \% Area & Area & Ret. Time & Height & Cap. Factor \\
\hline 1 & 94.8451 & 8407.2416 & $3.71 \mathrm{~min}$ & 852.508 & 0 \\
\hline 2 & 5.1549 & 456.9395 & $4.89 \mathrm{~min}$ & 23.4261 & 0 \\
\hline
\end{tabular}



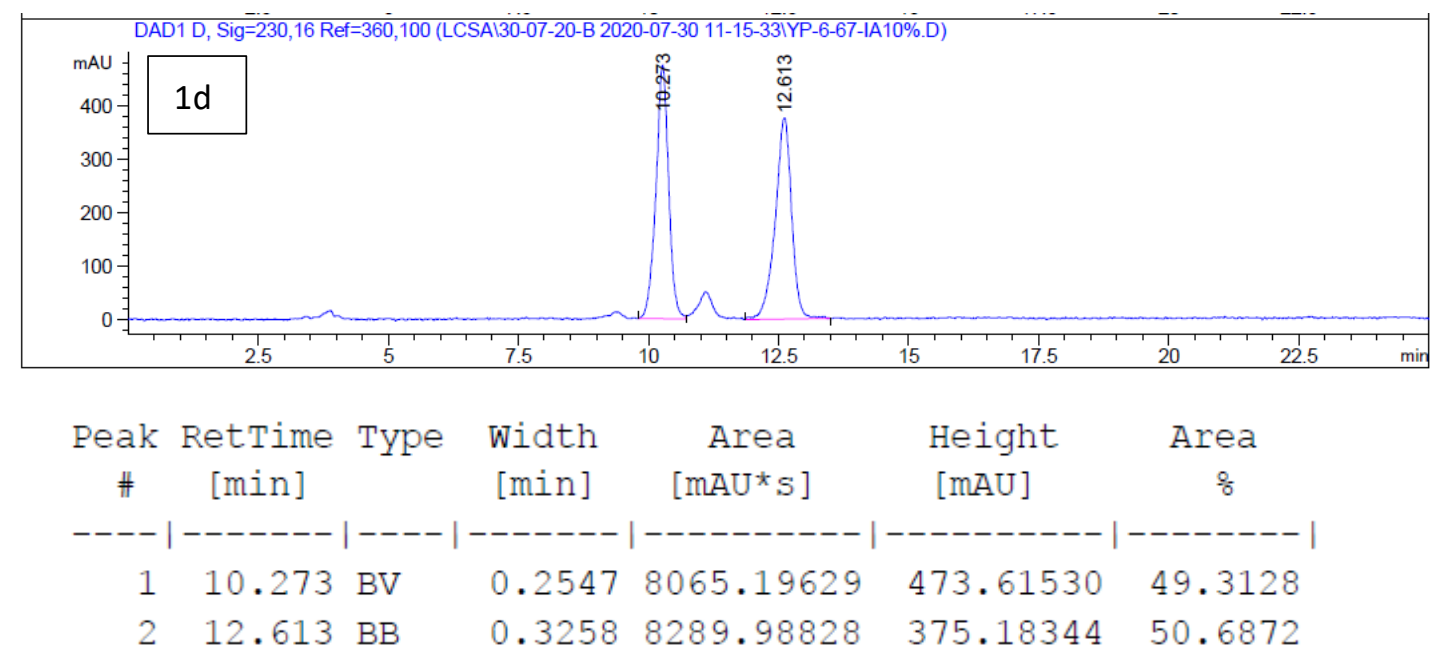

Totals :

$$
1.63552 \mathrm{e} 4 \quad 848.79874
$$
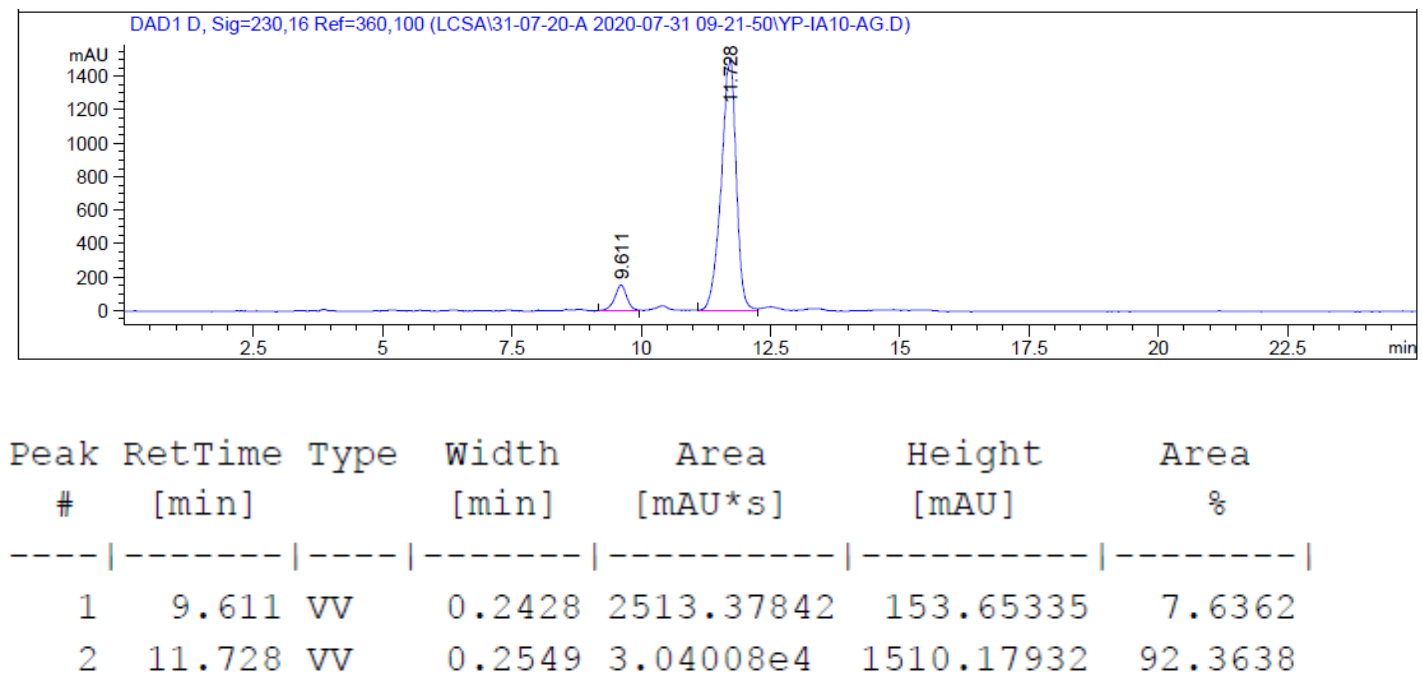

Totals :

$3.29142 e 4 \quad 1663.83267$ 


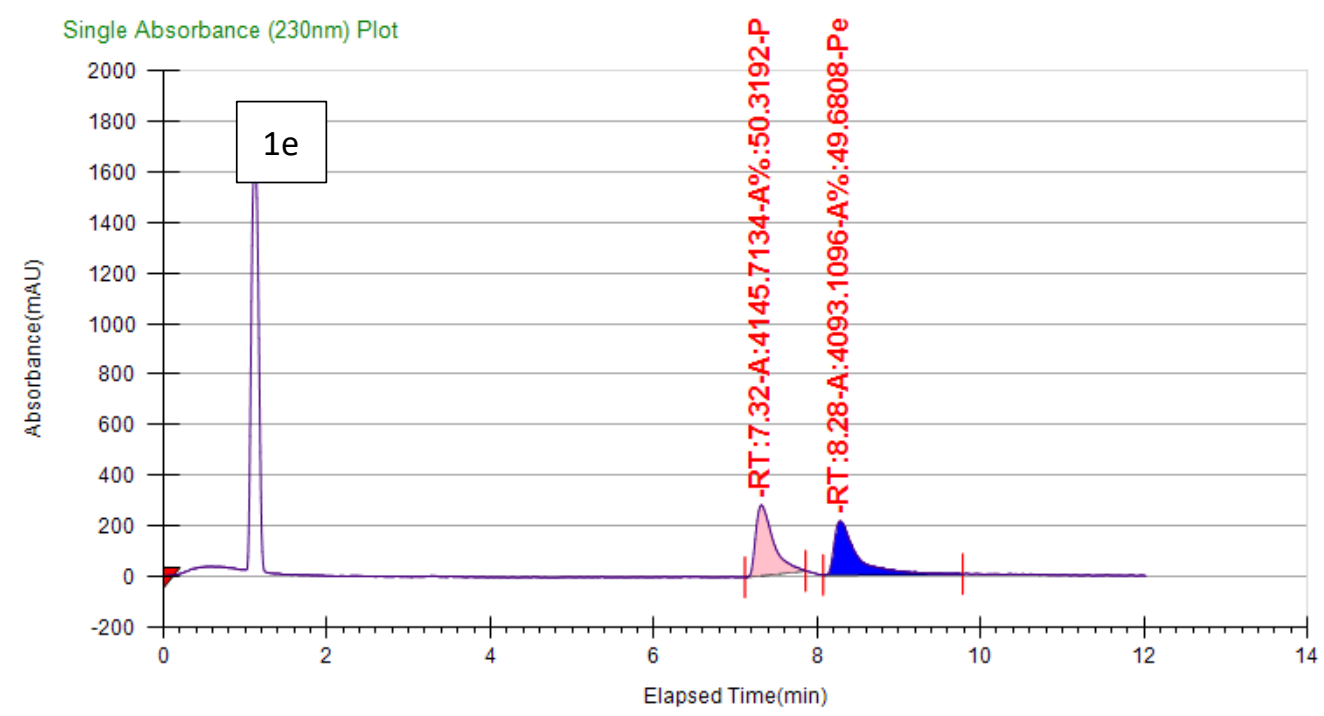

Run Information

\begin{tabular}{|l|l|l|l|l|l|l|l|l|l|}
\hline Instrument Method & Inj. Vol. & Solvent & Column & Sample & Well Location & Temp. & Flow & $\%$ Modifier & Pressure \\
\hline $5 p$ methanol & 10 & MeOH & IA & $\begin{array}{l}\text { yp-6- } \\
63^{\prime} \text { DL'IA5\% }\end{array}$ & $11 \mathrm{D}$ & 29.9 & 4 & 5 & 150 \\
\hline
\end{tabular}

Peak Information

\begin{tabular}{|l|l|l|l|l|l|}
\hline Peak No & \% Area & Area & Ret. Time & Height & Cap. Factor \\
\hline 1 & 50.3192 & 4145.7134 & $7.32 \mathrm{~min}$ & 278.2727 & 0 \\
\hline 2 & 49.6808 & 4093.1096 & $8.28 \mathrm{~min}$ & 212.9828 & 0 \\
\hline
\end{tabular}

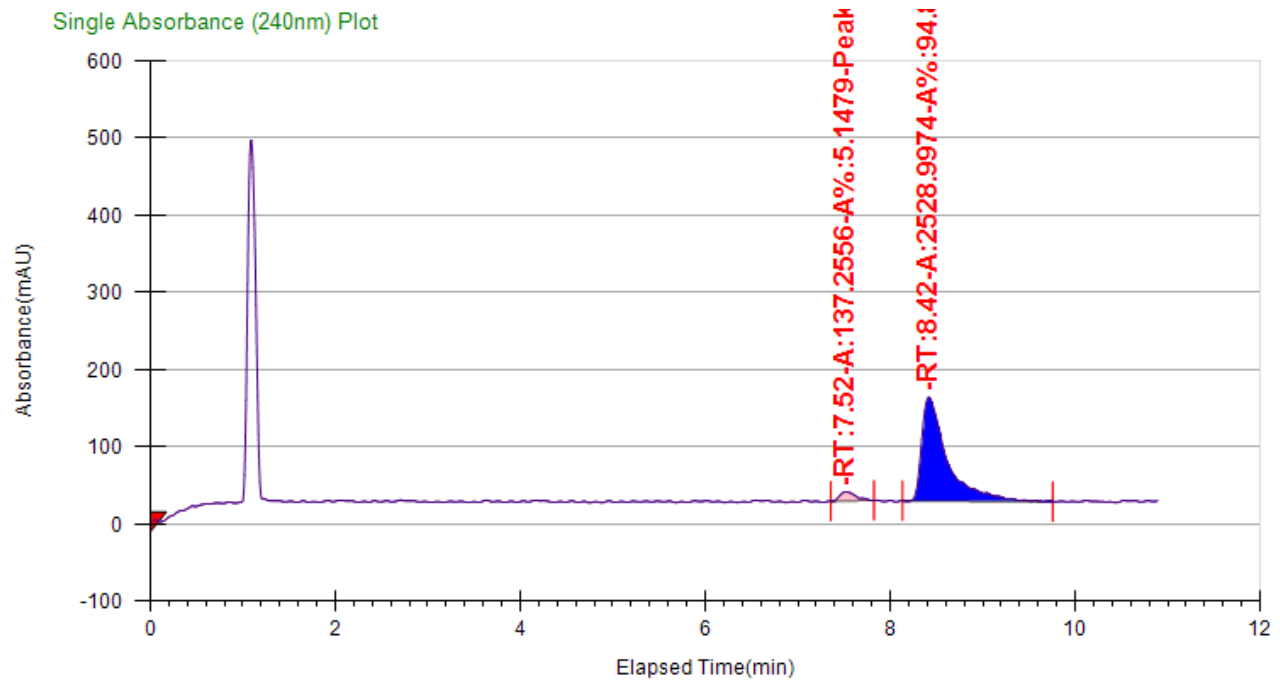

Run Information

\begin{tabular}{|l|l|l|l|l|l|l|l|l|l|}
\hline Instrument Method & Inj. Vol. & Solvent & Column & Sample & Well Location & Temp. & Flow & $\%$ Modifier & Pressure \\
\hline $5 p$ methanol & 10 & $\mathrm{MeOH}$ & IA & $\begin{array}{l}\text { yp-6-63-ASY- } \\
600 \mathrm{O}-\mathrm{IA} 5 \%\end{array}$ & $11 \mathrm{~F}$ & 30 & 4 & 5 & 150 \\
\hline
\end{tabular}

\section{Peak Information}

\begin{tabular}{|l|l|l|l|l|l|}
\hline Peak No & $\%$ Area & Area & Ret. Time & Height & Cap. Factor \\
\hline 1 & 5.1479 & 137.2556 & $7.52 \mathrm{~min}$ & 11.6486 & 0 \\
\hline 2 & 94.8521 & 2528.9974 & $8.42 \mathrm{~min}$ & 134.1054 & 0 \\
\hline
\end{tabular}




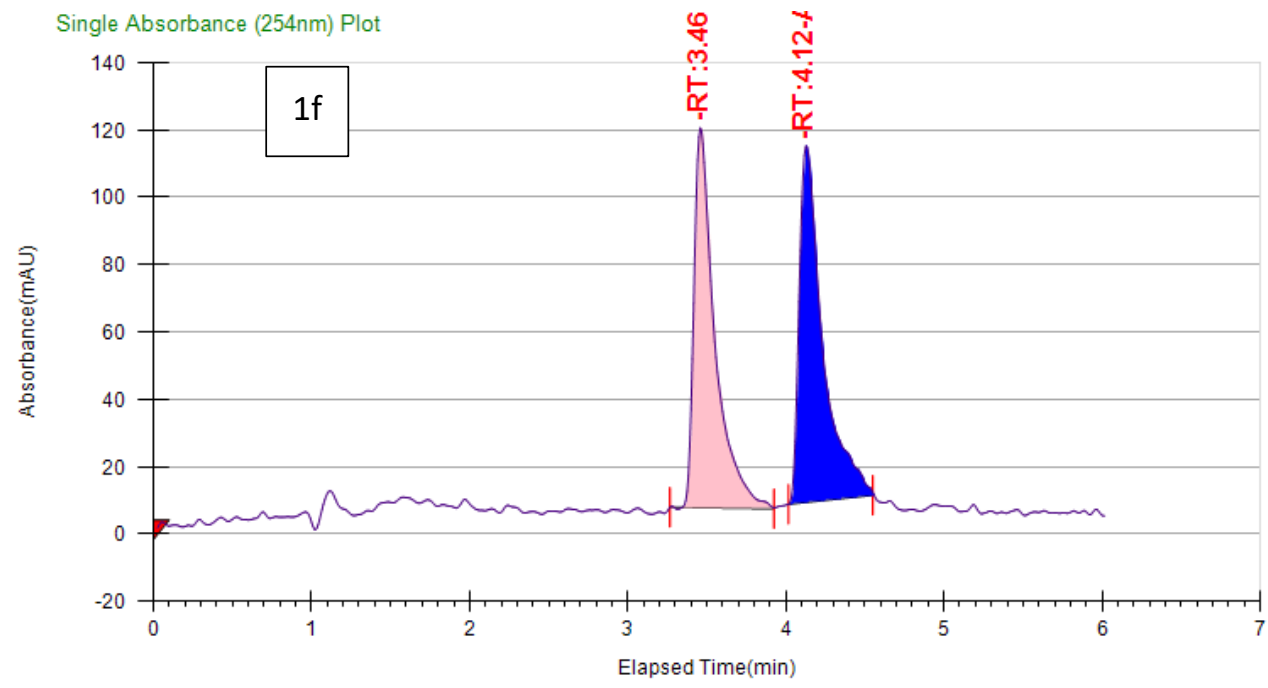

Run Information

\begin{tabular}{|l|l|l|l|l|l|l|l|l|l|}
\hline Instrument Method & Inj. Vol. & Solvent & Column & Sample & Well Location & Temp. & Flow & $\%$ Modifier & Pressure \\
\hline $5 p$ methanol & 10 & MeOH & IA & $\begin{array}{l}\text { yp-6-47-DL- } \\
\text { IA5\% }\end{array}$ & $11 \mathrm{~A}$ & 30.5 & 4 & 5 & 150 \\
\hline
\end{tabular}

Peak Information

\begin{tabular}{|l|l|l|l|l|l|}
\hline Peak No & $\%$ Area & Area & Ret. Time & Height & Cap. Factor \\
\hline 1 & 48.904 & 1031.079 & $3.46 \mathrm{~min}$ & 112.9079 & 0 \\
\hline 2 & 51.096 & 1077.2948 & $4.12 \mathrm{~min}$ & 105.8346 & 0 \\
\hline
\end{tabular}

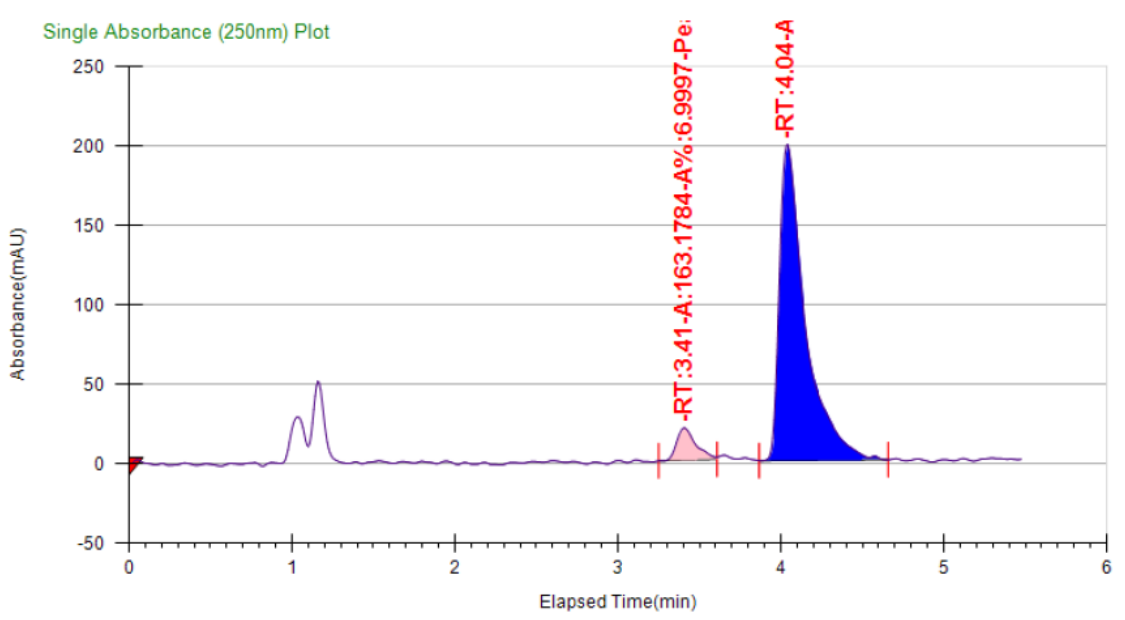

Run Information

\begin{tabular}{|l|l|l|l|l|l|l|l|l|l|}
\hline Instrument Method & Inj. Vol. & Solvent & Column & Sample & Well Location & Temp. & Flow & $\%$ Modifier & Pressure \\
\hline $5 p$ methanol & 10 & $\mathrm{MeOH}$ & IA & $\begin{array}{l}\text { yp-6-47-60 } \\
\text { oC-1day-IA5\% }\end{array}$ & $11 \mathrm{~B}$ & 29 & 4 & 5 & 150 \\
\hline
\end{tabular}

Peak Information

\begin{tabular}{|l|l|l|l|l|l|}
\hline Peak No & \% Area & Area & Ret. Time & Height & Cap. Factor \\
\hline 1 & 6.9997 & 163.1784 & $3.41 \mathrm{~min}$ & 20.3015 & 0 \\
\hline 2 & 93.0003 & 2168.0362 & $4.04 \mathrm{~min}$ & 198.9024 & 0 \\
\hline
\end{tabular}




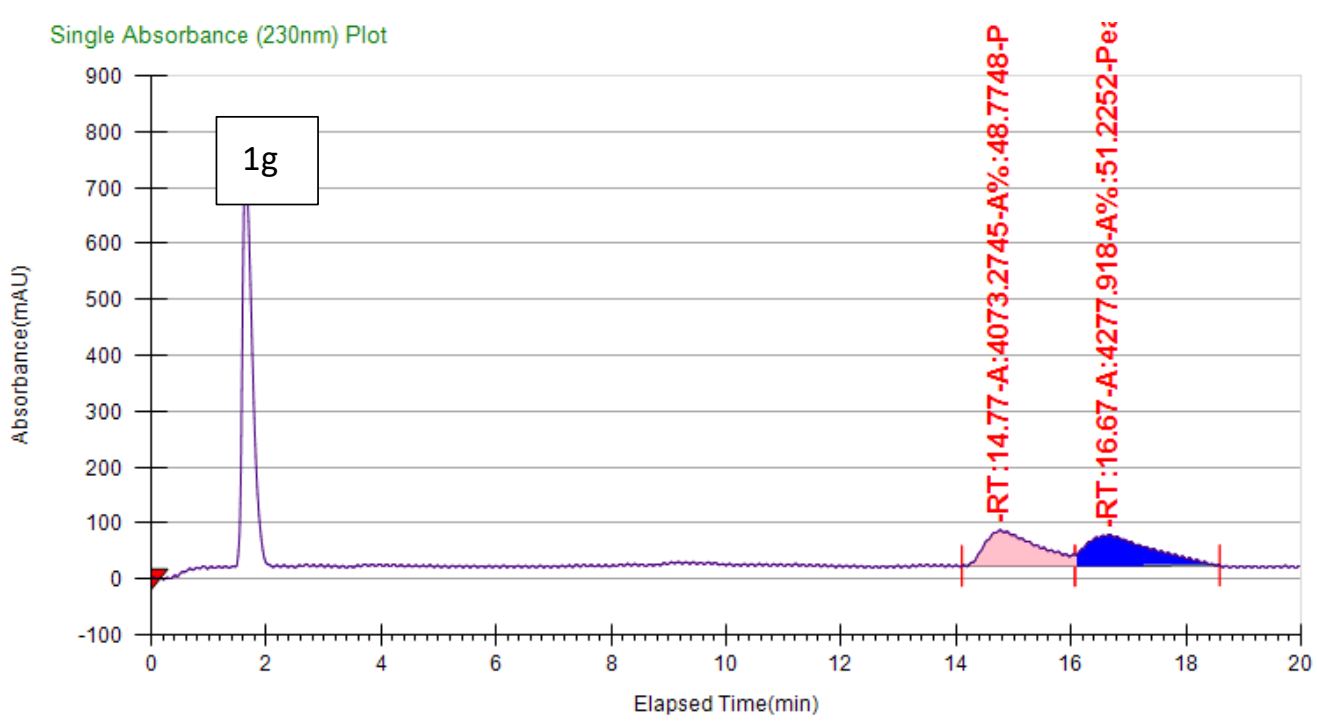

Run Information

\begin{tabular}{|l|l|l|l|l|l|l|l|l|l|}
\hline Instrument Method & Inj. Vol. & Solvent & Column & Sample & Well Location & Temp. & Flow & $\%$ Modifier & Pressure \\
\hline $1.5 p$ methanol & 10 & MeOH & IC & $\begin{array}{l}\text { yp-6-77-DL- } \\
\text { IC1.5\% }\end{array}$ & $11 \mathrm{E}$ & 30.3 & 4 & 1.5 & 150 \\
\hline
\end{tabular}

Peak Information

\begin{tabular}{|l|l|l|l|l|l|}
\hline Peak No & \% Area & Area & Ret. Time & Height & Cap. Factor \\
\hline 1 & 48.7748 & 4073.2745 & $14.77 \mathrm{~min}$ & 63.9159 & 0 \\
\hline 2 & 51.2252 & 4277.918 & $16.67 \mathrm{~min}$ & 55.9457 & 0 \\
\hline
\end{tabular}

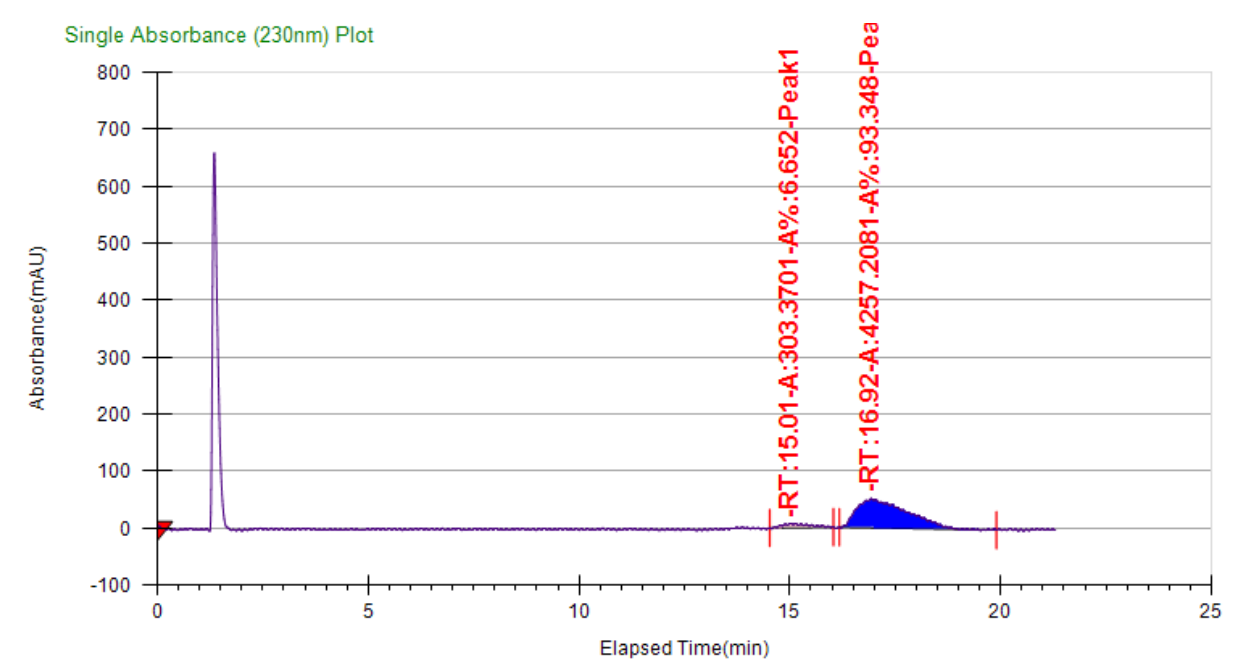

Run Information

\begin{tabular}{|l|l|l|l|l|l|l|l|l|l|}
\hline Instrument Method & Inj. Vol. & Solvent & Column & Sample & Well Location & Temp. & Flow & $\%$ Modifier & Pressure \\
\hline $1.5 p$ methanol & 10 & MeOH & IC & $\begin{array}{l}\text { yp-6-77-70oC-11E } \\
\text { asy-IC1.5\% }\end{array}$ & (I) & 29.9 & 4 & 1.5 & 150 \\
\hline
\end{tabular}

Peak Information

\begin{tabular}{|l|l|l|l|l|l|}
\hline Peak No & $\%$ Area & Area & Ret. Time & Height & Cap. Factor \\
\hline 1 & 6.652 & 303.3701 & $15.01 \mathrm{~min}$ & 7.4597 & 0 \\
\hline 2 & 93.348 & 4257.2081 & $16.92 \mathrm{~min}$ & 51.1444 & 0 \\
\hline
\end{tabular}




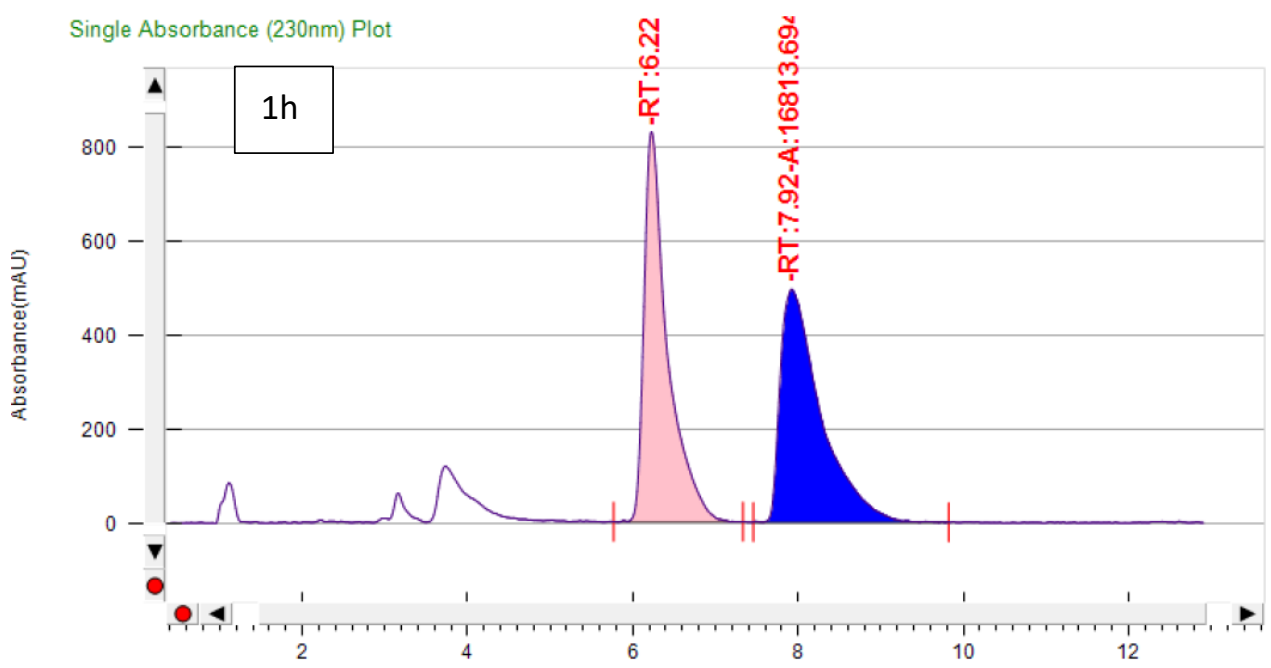

Run Information

\begin{tabular}{|l|l|l|l|l|l|l|l|l|l|}
\hline Instrument Method & Inj. Vol. & Solvent & Column & Sample & Well Location & Temp. & Flow & $\%$ Modifier & Pressure \\
\hline $15 p$ methanol & 10 & MeOH & OD-H & $\begin{array}{l}\text { yp-6-50-DL- } \\
\text { OD15\% }\end{array}$ & $11 \mathrm{~B}$ & 30.1 & 4 & 15 & 150 \\
\hline
\end{tabular}

Peak Information

\begin{tabular}{|l|l|l|l|l|l|}
\hline Peak No & $\%$ Area & Area & Ret. Time & Height & Cap. Factor \\
\hline 1 & 49.3758 & $\begin{array}{l}16399.056 \\
1\end{array}$ & $6.22 \mathrm{~min}$ & 832.0832 & 6223.9167 \\
\hline 2 & 50.6242 & $\begin{array}{l}16813.694 \\
9\end{array}$ & $7.92 \mathrm{~min}$ & 496.8907 & 7923.8833 \\
\hline
\end{tabular}

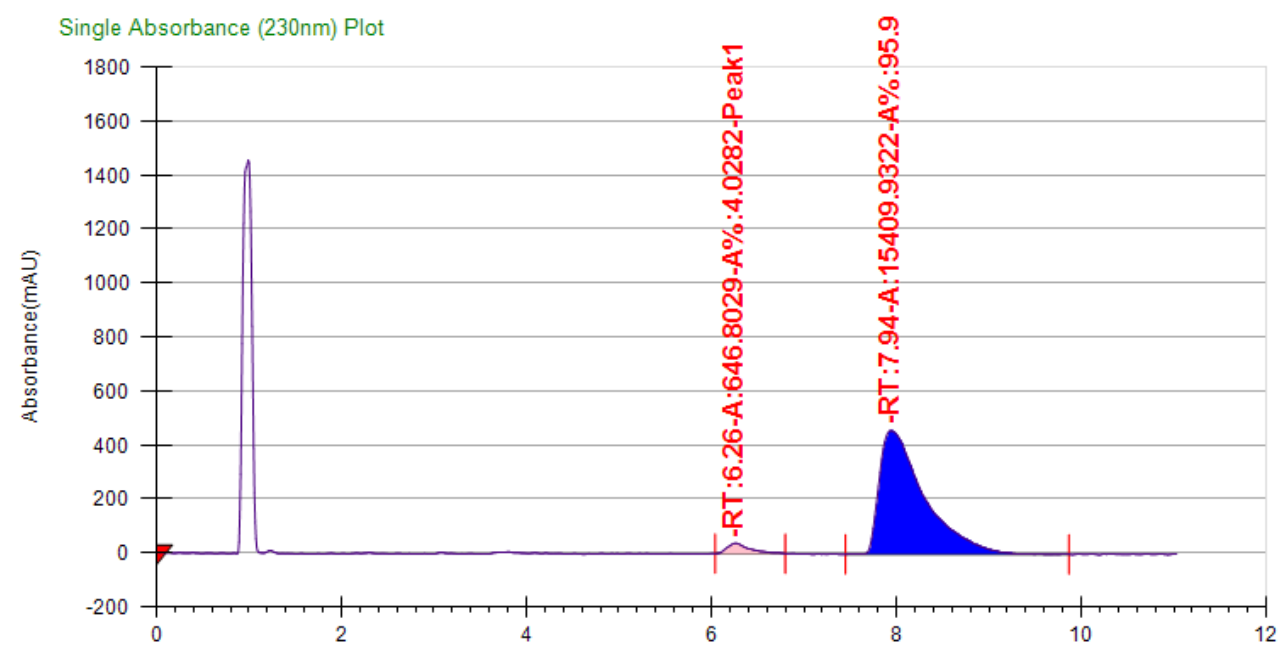

Run Information

\begin{tabular}{|l|l|l|l|l|l|l|l|l|l|}
\hline Instrument Method & Inj. Vol. & Solvent & Column & Sample & Well Location & Temp. & Flow & $\%$ Modifier & Pressure \\
\hline $15 p$ methanol & 10 & $\mathrm{MeOH}$ & OD-H & $\begin{array}{l}\text { yp-6-50-70oC-11A } \\
\text { day-OD15\% }\end{array}$ & 11.2 & 4 & 15 & 150 \\
\hline
\end{tabular}

\section{Peak Information}

\begin{tabular}{|l|l|l|l|l|l|}
\hline Peak No & $\%$ Area & Area & Ret. Time & Height & Cap. Factor \\
\hline 1 & 4.0282 & 646.8029 & $6.26 \mathrm{~min}$ & 38.4597 & 0 \\
\hline 2 & 95.9718 & $\begin{array}{l}15409.932 \\
2\end{array}$ & $7.94 \mathrm{~min}$ & 458.1345 & 0 \\
\hline
\end{tabular}




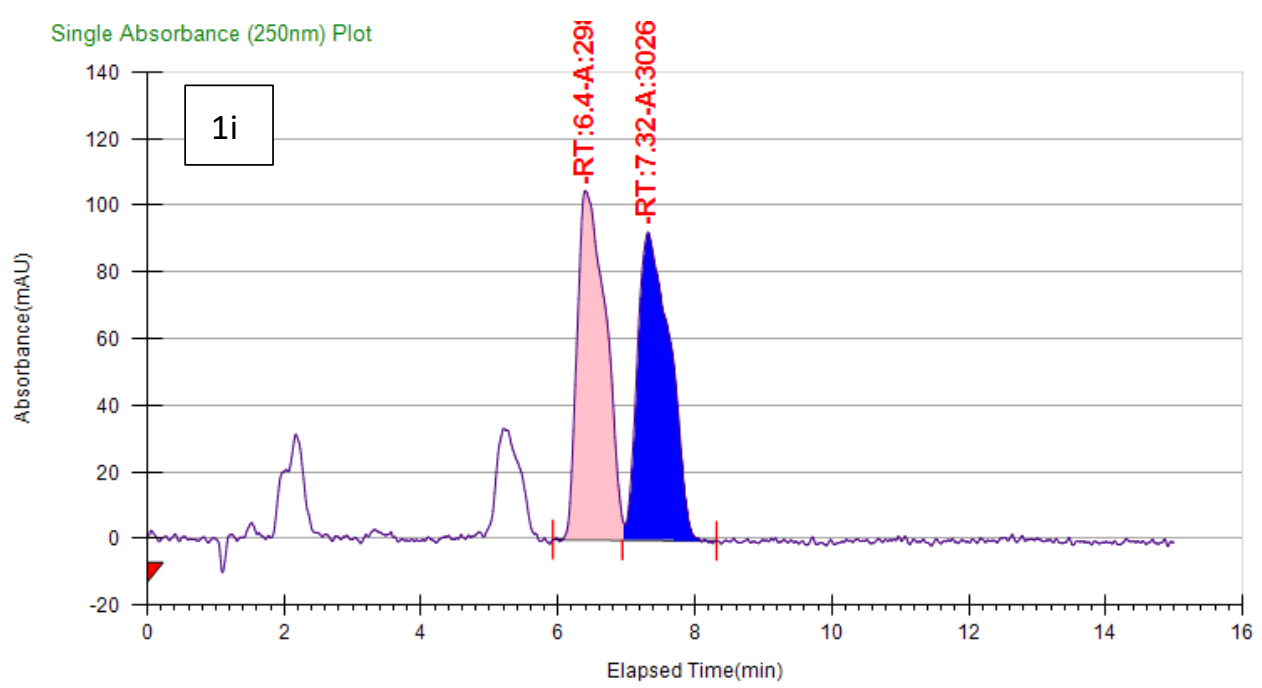

Run Information

\begin{tabular}{|l|l|l|l|l|l|l|l|l|l|}
\hline Instrument Method & Inj. Vol. & Solvent & Column & Sample & Well Location & Temp. & Flow & $\%$ Modifier & Pressure \\
\hline 10p methanol & 10 & $\mathrm{MeOH}$ & IC & $\begin{array}{l}\text { yp-6-68-DL- } \\
\text { IC } 10 \%\end{array}$ & $11 \mathrm{D}$ & 30 & 4 & 10 & 150 \\
\hline
\end{tabular}

Peak Information

\begin{tabular}{|l|l|l|l|l|l|}
\hline Peak No & $\%$ Area & Area & Ret. Time & Height & Cap. Factor \\
\hline 1 & 49.683 & 2988.2711 & $6.4 \mathrm{~min}$ & 104.8604 & 6398.9167 \\
\hline 2 & 50.317 & 3026.4062 & $7.32 \mathrm{~min}$ & 92.5598 & 7315.5667 \\
\hline
\end{tabular}

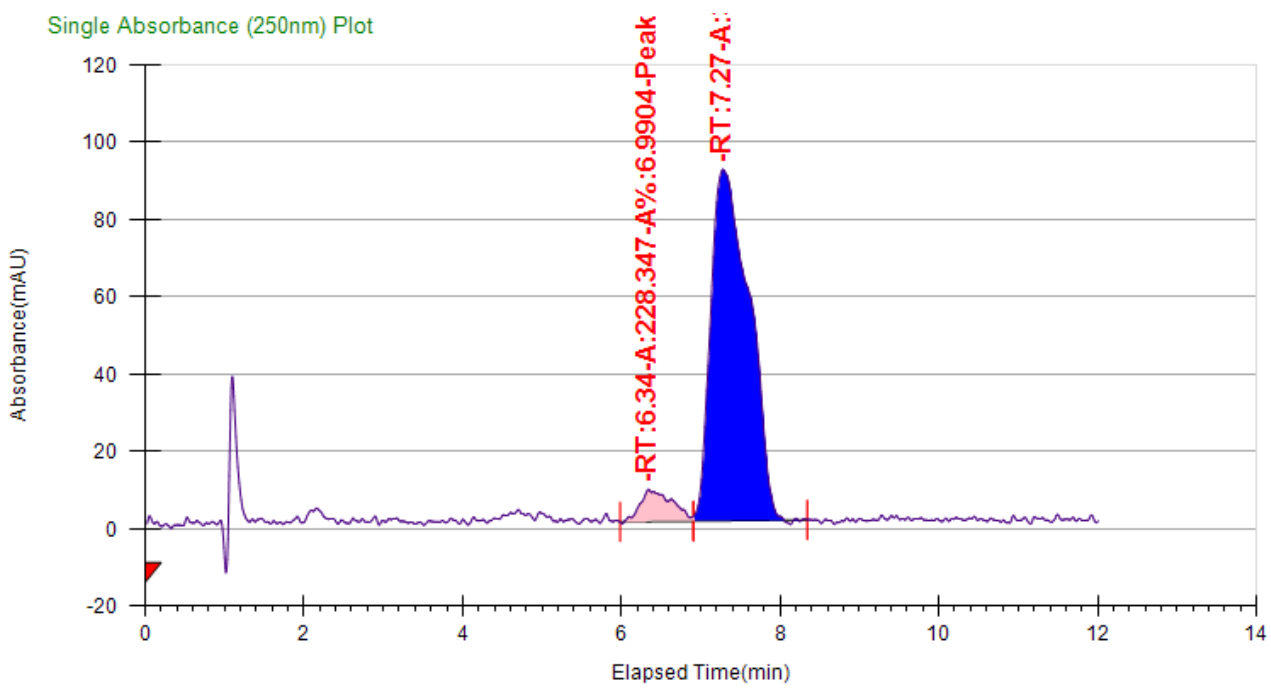

Run Information

\begin{tabular}{|l|l|l|l|l|l|l|l|l|l|}
\hline Instrument Method & Inj. Vol. & Solvent & Column & Sample & Well Location & Temp. & Flow & $\%$ Modifier & Pressure \\
\hline $10 \mathrm{p}$ methanol & 10 & $\mathrm{MeOH}$ & IC & $\begin{array}{l}\text { yp-6-68-ASY- } \\
\text { IC10\% }\end{array}$ & $11 \mathrm{C}$ & 29.8 & 4 & 10 & 150 \\
\hline
\end{tabular}

\section{Peak Information}

\begin{tabular}{|l|l|l|l|l|l|}
\hline Peak No & $\%$ Area & Area & Ret. Time & Height & Cap. Factor \\
\hline 1 & 6.9904 & 228.347 & $6.34 \mathrm{~min}$ & 8.3675 & 0 \\
\hline 2 & 93.0096 & 3038.2117 & $7.27 \mathrm{~min}$ & 90.9961 & 0 \\
\hline
\end{tabular}




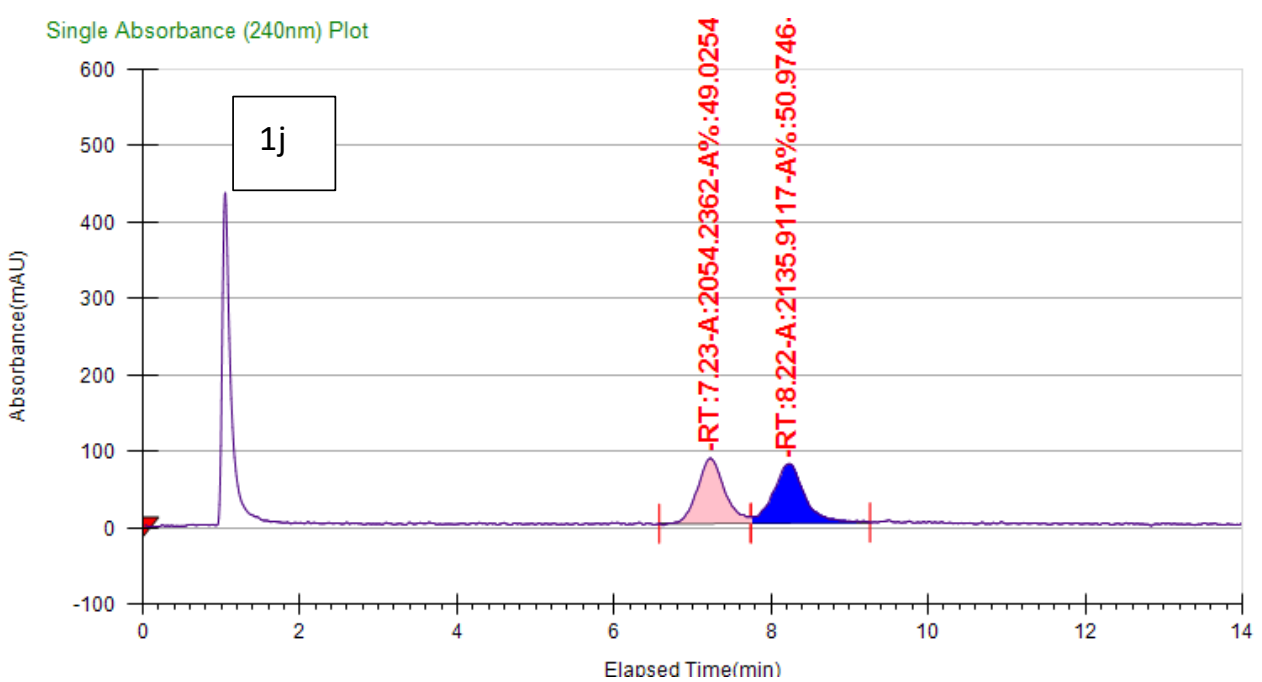

Run Information

\begin{tabular}{|l|l|l|l|l|l|l|l|l|l|}
\hline Instrument Method & Inj. Vol. & Solvent & Column & Sample & Well Location & Temp. & Flow & \% Modifier & Pressure \\
\hline $5 p$ methanol & 10 & $\mathrm{MeOH}$ & AD-H & $\begin{array}{l}\text { yp-6-62-DL- } \\
\text { AD5\% }\end{array}$ & $11 \mathrm{C}$ & 30 & 4 & 5 & 150 \\
\hline
\end{tabular}

Peak Information

\begin{tabular}{|l|l|l|l|l|l|}
\hline Peak No & $\%$ Area & Area & Ret. Time & Height & Cap. Factor \\
\hline 1 & 49.0254 & 2054.2362 & $7.23 \mathrm{~min}$ & 85.1274 & 0 \\
\hline 2 & 50.9746 & 2135.9117 & $8.22 \mathrm{~min}$ & 77.3448 & 0 \\
\hline
\end{tabular}

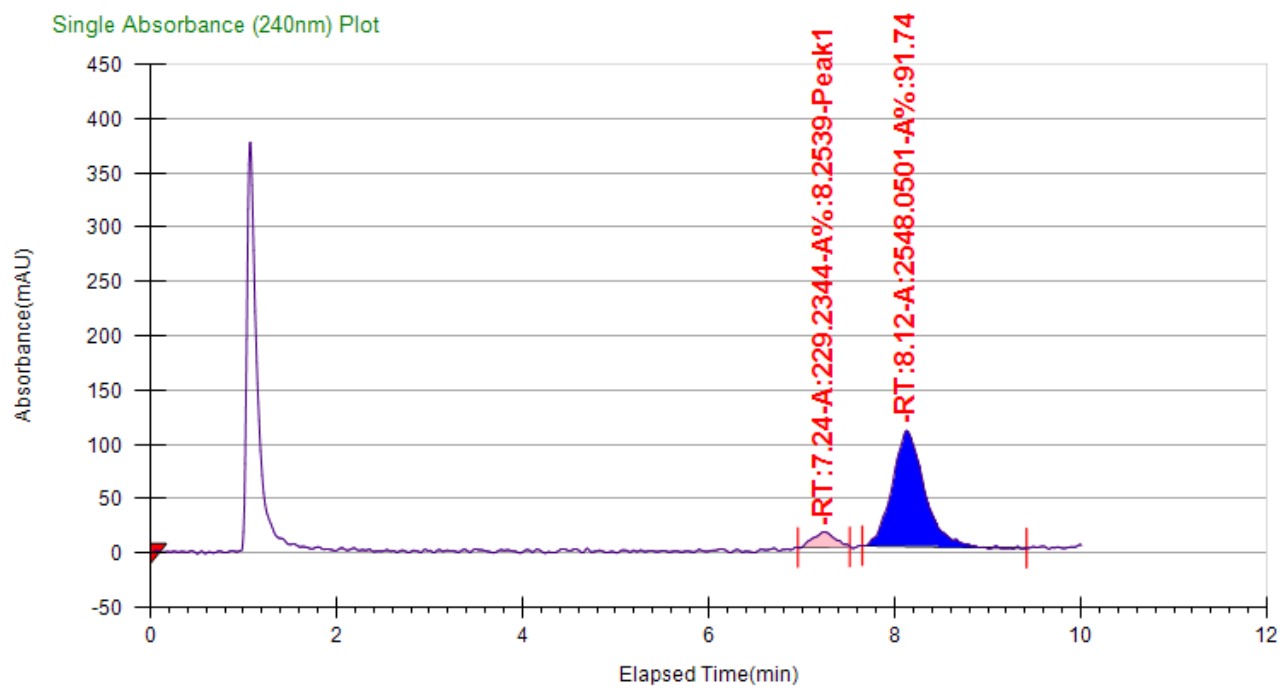

Run Information

\begin{tabular}{|l|l|l|l|l|l|l|l|l|l|}
\hline Instrument Method & Inj. Vol. & Solvent & Column & Sample & Well Location & Temp. & Flow & $\%$ Modifier & Pressure \\
\hline $5 p$ methanol & 10 & $\mathrm{MeOH}$ & AD-H & $\begin{array}{l}\text { yp-6-62-ASY- } \\
700 C-A D 5 \%\end{array}$ & $11 \mathrm{~A}$ & 29.9 & 4 & 5 & 150 \\
\hline
\end{tabular}

Peak Information

\begin{tabular}{|l|l|l|l|l|l|}
\hline Peak No & $\%$ Area & Area & Ret. Time & Height & Cap. Factor \\
\hline 1 & 8.2539 & 229.2344 & $7.24 \mathrm{~min}$ & 14.1165 & 7240.5667 \\
\hline 2 & 91.7461 & 2548.0501 & $8.12 \mathrm{~min}$ & 106.5392 & 8123.8833 \\
\hline
\end{tabular}




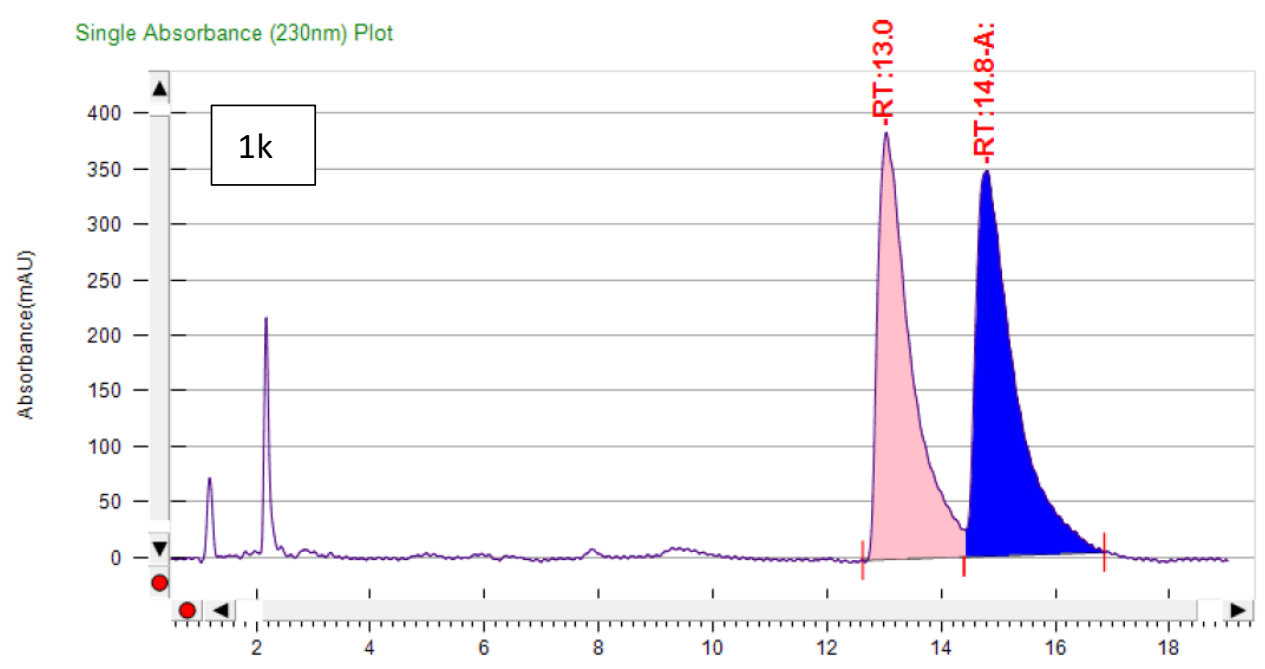

Run Information

\begin{tabular}{|l|l|l|l|l|l|l|l|l|l|}
\hline Instrument Method & Inj. Vol. & Solvent & Column & Sample & Well Location & Temp. & Flow & $\%$ Modifier & Pressure \\
\hline $10 \mathrm{p}$ methanol & 10 & $\mathrm{MeOH}$ & IA & $\begin{array}{l}6-988^{\prime} \mathrm{DL}- \\
\text { IA10\% }\end{array}$ & $11 \mathrm{D}$ & 30.1 & 4 & 10 & 150 \\
\hline
\end{tabular}

\section{Peak Information}

\begin{tabular}{|l|l|l|l|l|l|}
\hline Peak No & $\%$ Area & Area & Ret. Time & Height & Cap. Factor \\
\hline 1 & 48.8505 & $\begin{array}{l}15600.405 \\
6\end{array}$ & $13.03 \mathrm{~min}$ & 383.915 & 13032.15 \\
\hline 2 & 51.1495 & $\begin{array}{l}16334.616 \\
7\end{array}$ & $14.8 \mathrm{~min}$ & 346.6891 & 14798.7833 \\
\hline
\end{tabular}

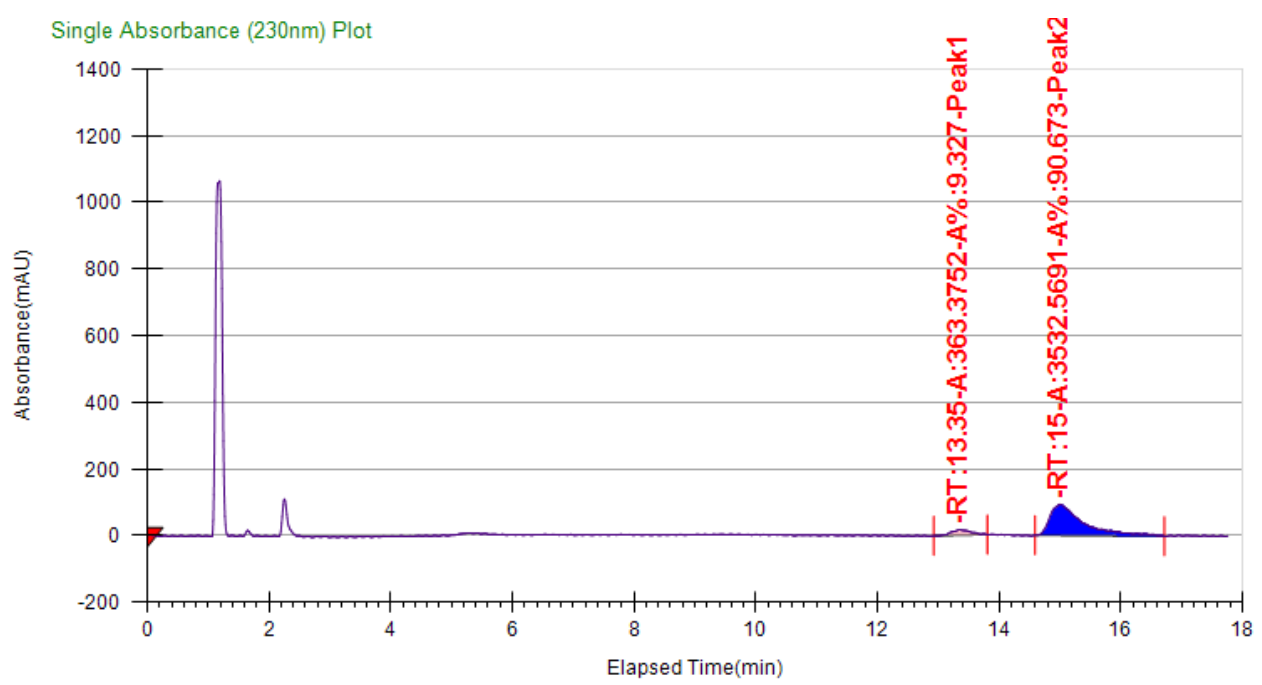

Run Information

\begin{tabular}{|c|c|c|c|c|c|c|c|c|c|}
\hline Instrument Method & Inj. Vol. & Solvent & Column & Sample & Well Location & Temp. & Flow & $\%$ Modifier & Pressure \\
\hline 10p methanol & 10 & $\mathrm{MeOH}$ & IA & $\begin{array}{l}\text { 6-98-asy- } \\
\text { 20\%PA- } \\
\text { 4h'|A10\% }\end{array}$ & 11D & 31.3 & 4 & 10 & 150 \\
\hline
\end{tabular}

Peak Information

\begin{tabular}{|l|l|l|l|l|l|}
\hline Peak No & \% Area & Area & Ret. Time & Height & Cap. Factor \\
\hline 1 & 9.327 & 363.3752 & $13.35 \mathrm{~min}$ & 16.4597 & 13348.8 \\
\hline 2 & 90.673 & 3532.5691 & $15 \mathrm{~min}$ & 93.1456 & 14998.7833 \\
\hline
\end{tabular}




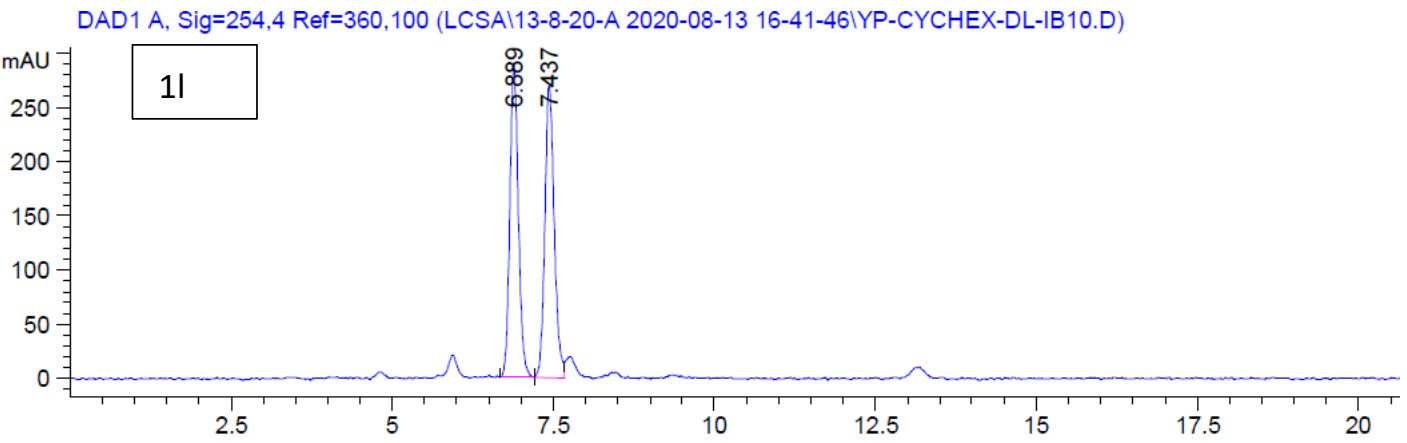

\begin{tabular}{|c|c|c|c|c|c|c|}
\hline eak & $\begin{array}{c}\text { RetTime } \\
\text { [min] }\end{array}$ & Type & $\begin{array}{l}\text { Width } \\
\text { [min] }\end{array}$ & $\begin{array}{c}\text { Area } \\
{\left[\mathrm{mAU}^{*} \mathrm{~s}\right]}\end{array}$ & $\begin{array}{l}\text { Height } \\
{[\mathrm{mAU}]}\end{array}$ & $\begin{array}{c}\text { Area } \\
\frac{8}{6}\end{array}$ \\
\hline & & & & 1 & & 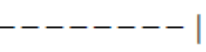 \\
\hline 1 & & & & 2672 . & 28 & 697 \\
\hline 2 & 37 & . & & 2718.80078 & 269.56525 & 4303 \\
\hline
\end{tabular}

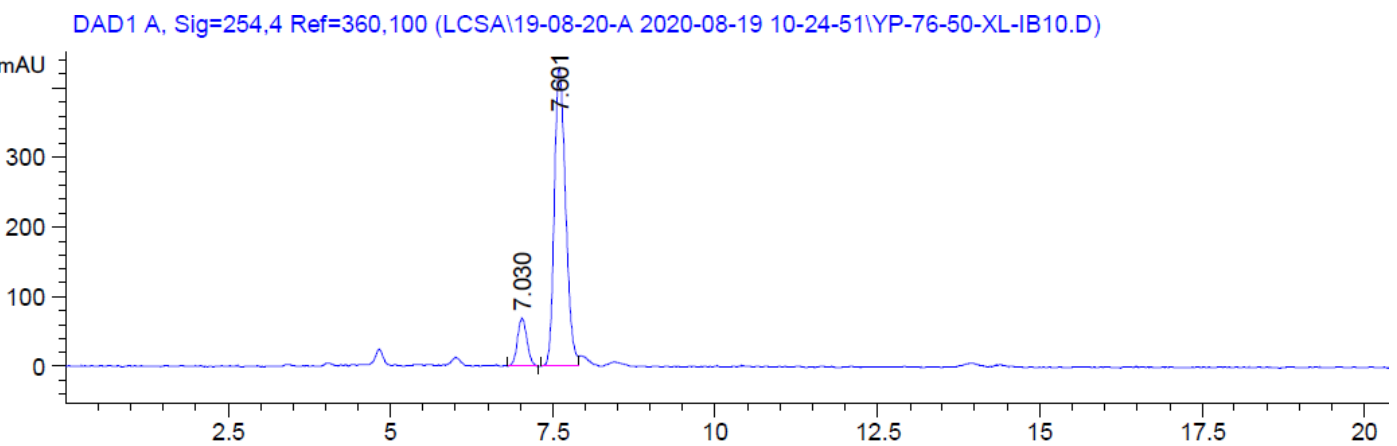

\begin{tabular}{|c|c|c|c|c|c|c|}
\hline $\begin{array}{c}\text { eak } \\
\#\end{array}$ & $\begin{array}{c}\text { RetTime } \\
\text { [min] }\end{array}$ & Type & $\begin{array}{l}\text { Width } \\
\text { [min] }\end{array}$ & $\begin{array}{c}\text { Area } \\
{\left[\mathrm{mAU}^{*} \mathrm{~S}\right]}\end{array}$ & $\begin{array}{l}\text { Height } \\
{[\mathrm{mAU}]}\end{array}$ & $\begin{array}{c}\text { Area } \\
\frac{8}{8}\end{array}$ \\
\hline & & & & 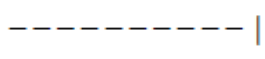 & . & \\
\hline 1 & & & & 698 & 583 & 88 \\
\hline 2 & 7.601 & 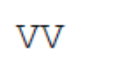 & 91 & 5186.09326 & 428.87134 & 1312 \\
\hline
\end{tabular}




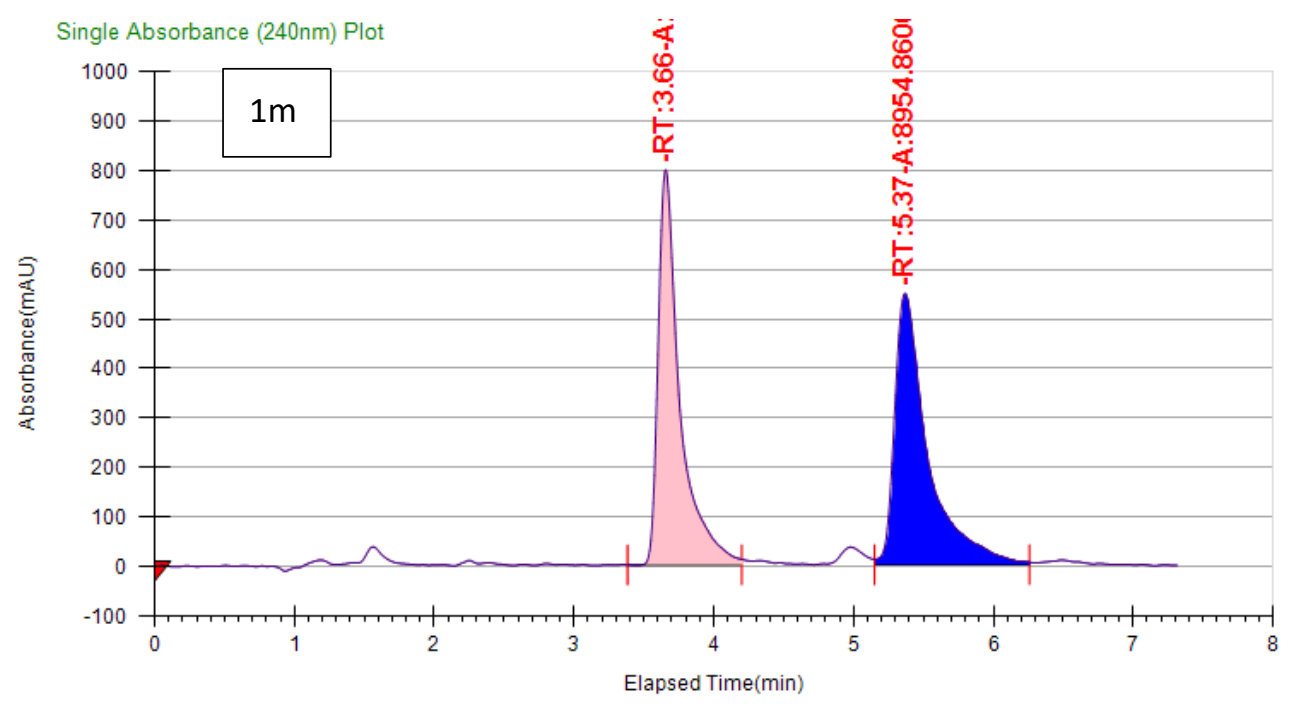

Run Information

\begin{tabular}{|l|l|l|l|l|l|l|l|l|l|}
\hline Instrument Method & Inj. Vol. & Solvent & Column & Sample & Well Location & Temp. & Flow & \% Modifier & Pressure \\
\hline 12p methanol & 10 & $\mathrm{MeOH}$ & OJ-H & $\begin{array}{l}\text { yp-hwu-6-26- } \\
\text { 4F-dl-OJ12\% }\end{array}$ & $11 \mathrm{~F}$ & 30.1 & 4 & 12 & 150 \\
\hline
\end{tabular}

Peak Information

\begin{tabular}{|l|l|l|l|l|l|}
\hline Peak No & $\%$ Area & Area & Ret. Time & Height & Cap. Factor \\
\hline 1 & 48.882 & 8563.16 & $3.66 \mathrm{~min}$ & 797.8752 & 3657.2833 \\
\hline 2 & 51.118 & 8954.8606 & $5.37 \mathrm{~min}$ & 548.2833 & 5365.6 \\
\hline
\end{tabular}




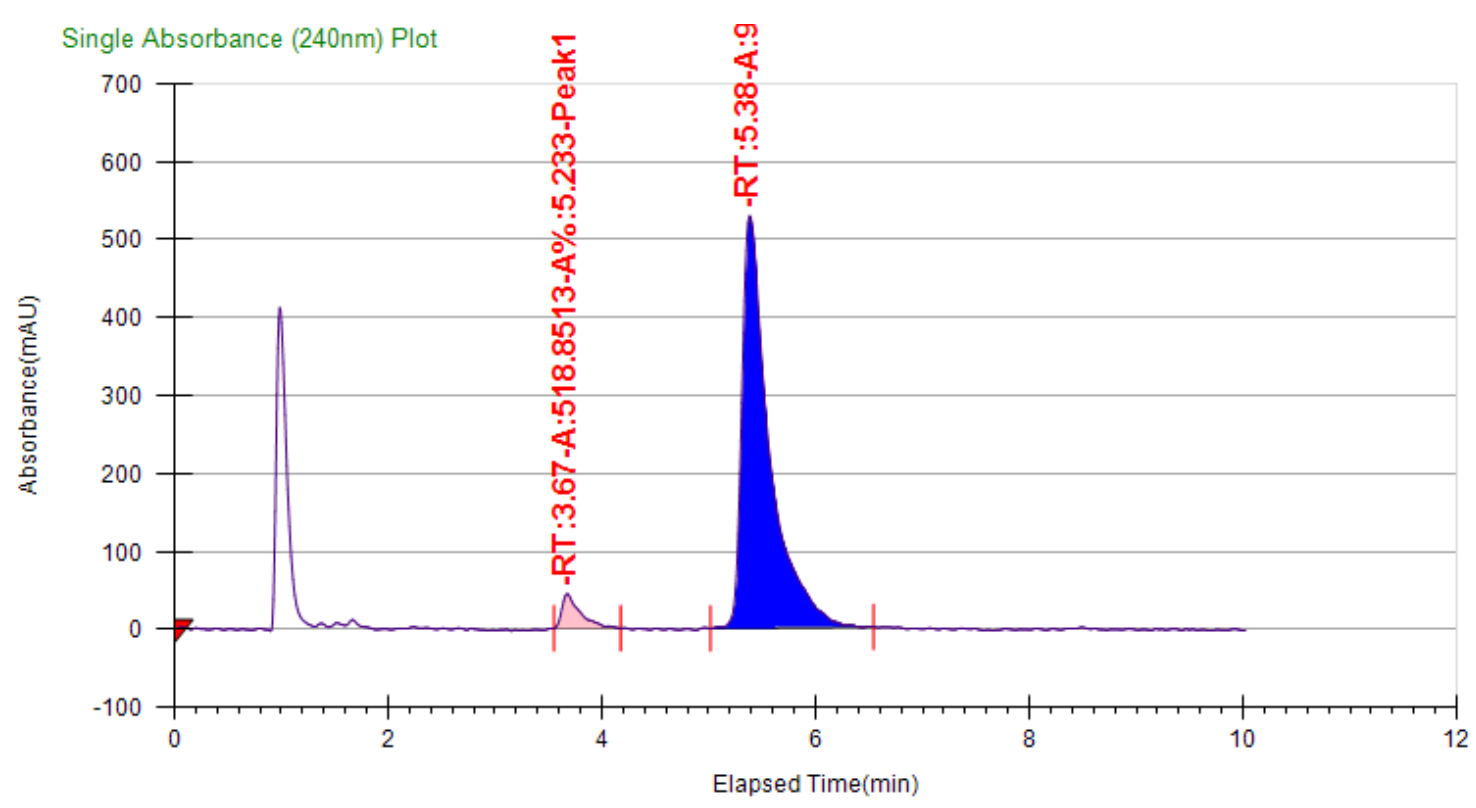

Run Information

\begin{tabular}{|l|l|l|l|l|l|l|l|l|l|}
\hline Instrument Method & Inj. Vol. & Solvent & Column & Sample & Well Location & Temp. & Flow & $\%$ Modifier & Pressure \\
\hline $12 \mathrm{p}$ methanol & 10 & $\mathrm{MeOH}$ & OJ-H & $\begin{array}{l}\text { yp-hwu-6-29- } \\
\text { 4F'asy- } \\
\text { OJ12\% }\end{array}$ & $11 \mathrm{~A}$ & 30 & 4 & 12 & 150 \\
\hline
\end{tabular}

\section{Peak Information}

\begin{tabular}{|l|l|l|l|l|l|}
\hline Peak No & $\%$ Area & Area & Ret. Time & Height & Cap. Factor \\
\hline 1 & 5.233 & 518.8513 & $3.67 \mathrm{~min}$ & 44.1196 & 3673.95 \\
\hline 2 & 94.767 & 9396.1994 & $5.38 \mathrm{~min}$ & 529.1197 & 5382.2667 \\
\hline
\end{tabular}




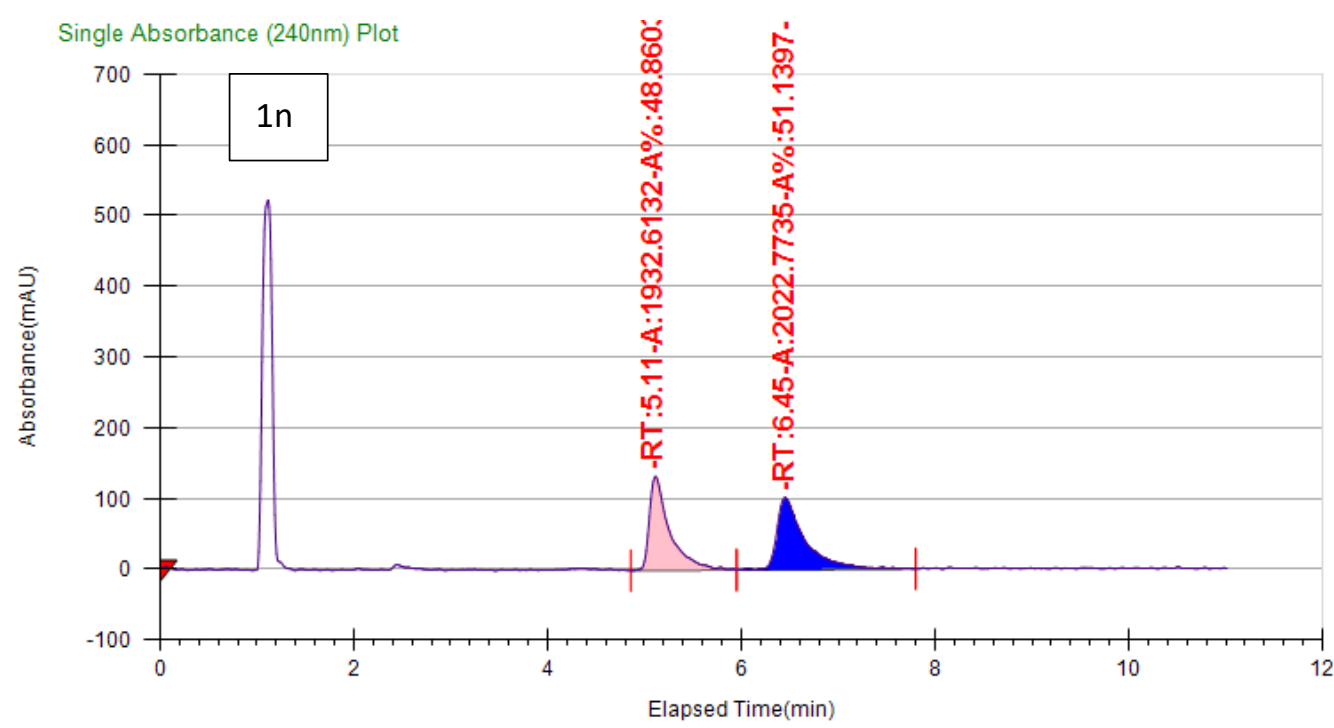

Run Information

\begin{tabular}{|l|l|l|l|l|l|l|l|l|l|}
\hline Instrument Method & Inj. Vol. & Solvent & Column & Sample & Well Location & Temp. & Flow & $\%$ Modifier & Pressure \\
\hline $10 p$ methanol & 10 & MeOH & IA & $\begin{array}{l}\text { YP-6-43-DL- } \\
\text { IA10\% }\end{array}$ & $11 \mathrm{~B}$ & 30 & 4 & 10 & 150 \\
\hline
\end{tabular}

Peak Information

\begin{tabular}{|l|l|l|l|l|l|}
\hline Peak No & \% Area & Area & Ret. Time & Height & Cap. Factor \\
\hline 1 & 48.8603 & 1932.6132 & $5.11 \mathrm{~min}$ & 132.8477 & 0 \\
\hline 2 & 51.1397 & 2022.7735 & $6.45 \mathrm{~min}$ & 101.863 & 0 \\
\hline
\end{tabular}

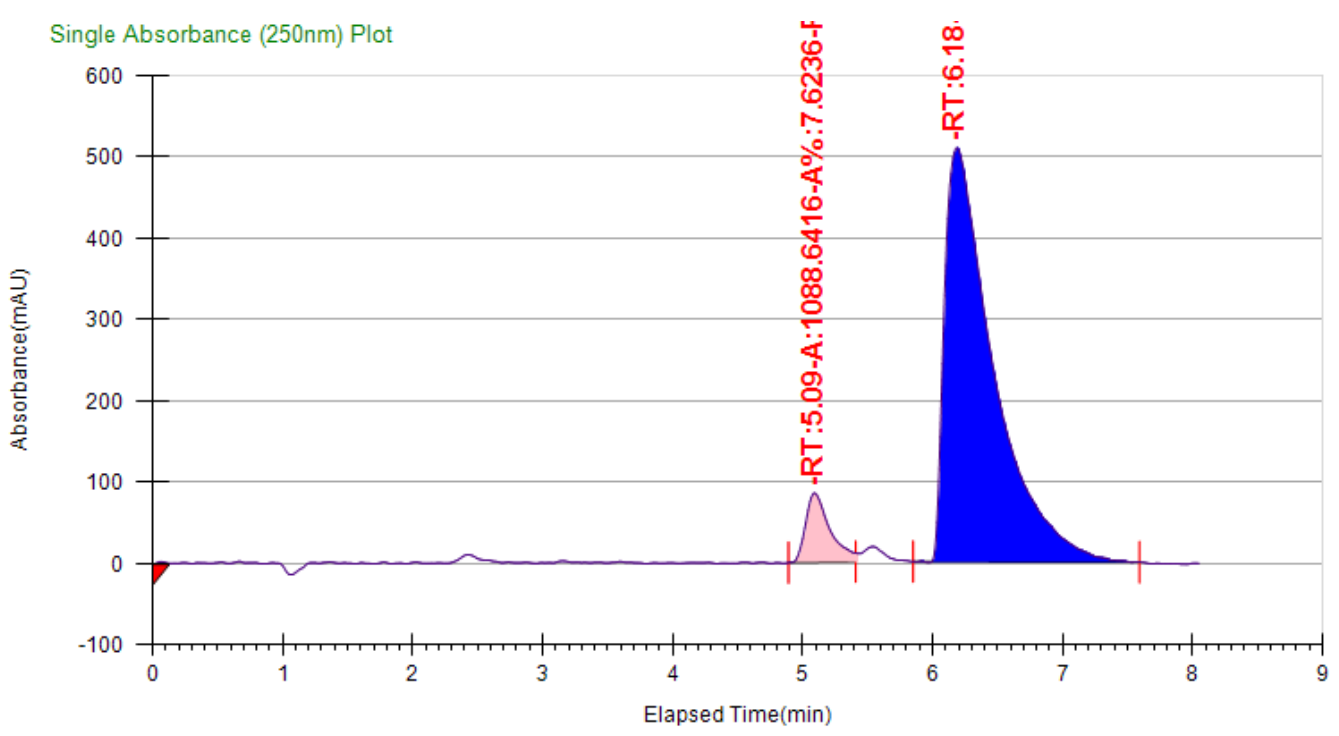

Run Information

\begin{tabular}{|l|l|l|l|l|l|l|l|l|l|}
\hline Instrument Method & Inj. Vol. & Solvent & Column & Sample & Well Location & Temp. & Flow & \% Modifier & Pressure \\
\hline $10 p$ methanol & 10 & MeOH & IA & $\begin{array}{l}\text { YP-6-43-asy- } \\
\text { IA10\% }\end{array}$ & $11 \mathrm{~A}$ & 30.1 & 4 & 10 & 150 \\
\hline
\end{tabular}

Peak Information

\begin{tabular}{|l|l|l|l|l|l|}
\hline Peak No & $\%$ Area & Area & Ret. Time & Height & Cap. Factor \\
\hline 1 & 7.6236 & 1088.6416 & $5.09 \mathrm{~min}$ & 85.1228 & 0 \\
\hline 2 & 92.3764 & 13191.171 & $6.18 \mathrm{~min}$ & 509.1165 & 0 \\
9 & & & & \\
\hline
\end{tabular}




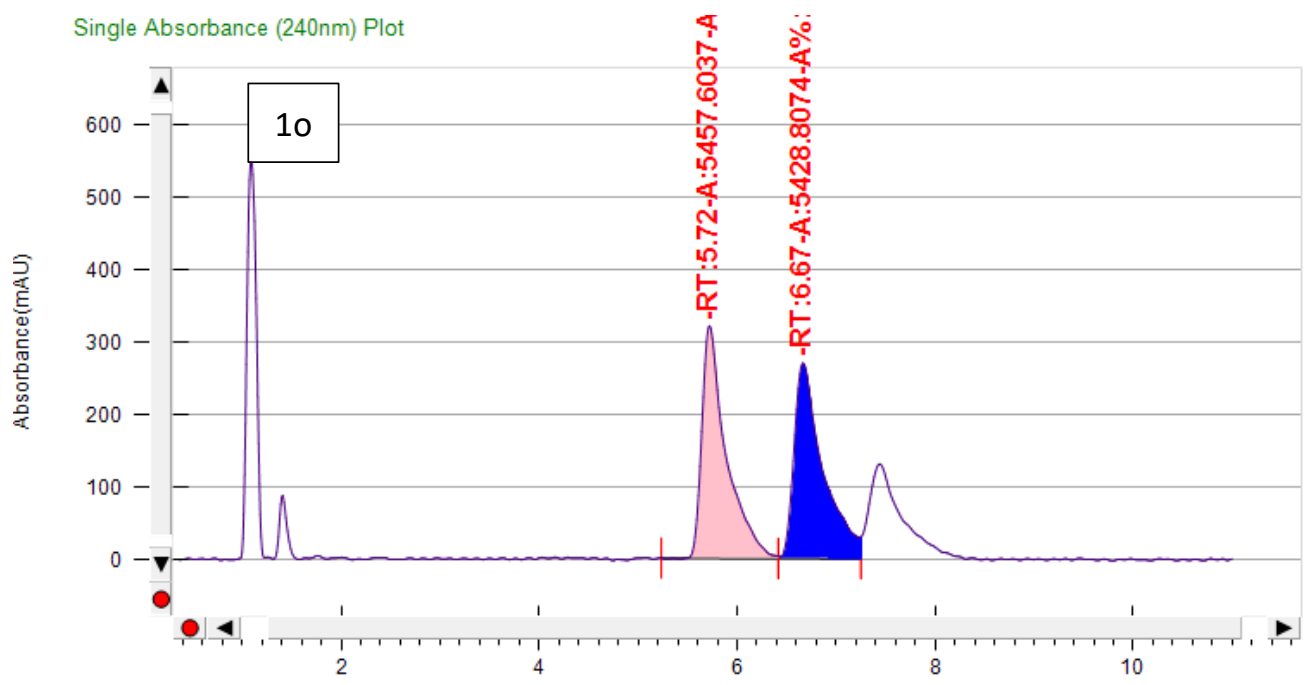

Run Information

\begin{tabular}{|l|l|l|l|l|l|l|l|l|l|}
\hline Instrument Method & Inj. Vol. & Solvent & Column & Sample & Well Location & Temp. & Flow & $\%$ Modifier & Pressure \\
\hline $5 p$ methanol & 10 & $\mathrm{MeOH}$ & OD-H & $\begin{array}{l}\text { YP-4-44-DL- } \\
\text { OD5\% }\end{array}$ & $11 \mathrm{~B}$ & 30.1 & 4 & 5 & 150 \\
\hline
\end{tabular}

Peak Information

\begin{tabular}{|l|l|l|l|l|l|}
\hline Peak No & $\%$ Area & Area & Ret. Time & Height & Cap. Factor \\
\hline 1 & 50.1323 & 5457.6037 & $5.72 \mathrm{~min}$ & 321.6424 & 5715.5833 \\
\hline 2 & 49.8677 & 5428.8074 & $6.67 \mathrm{~min}$ & 270.8836 & 6665.5667 \\
\hline
\end{tabular}

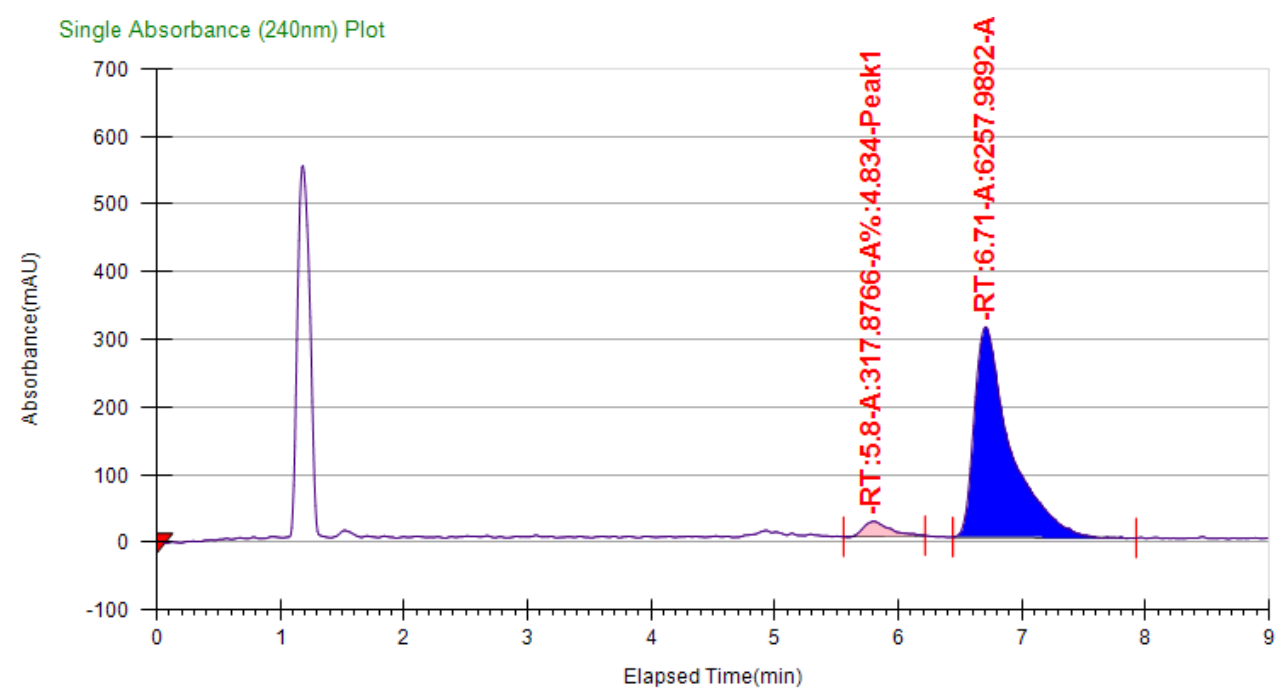

Run Information

\begin{tabular}{|l|l|l|l|l|l|l|l|l|l|}
\hline Instrument Method & Inj. Vol. & Solvent & Column & Sample & Well Location & Temp. & Flow & $\%$ Modifier & Pressure \\
\hline $5 p$ methanol & 10 & MeOH & OD-H & $\begin{array}{l}\text { yp-6-44- } \\
15 \% \text { PA-2day- } \\
\text { OD5\% }\end{array}$ & $11 \mathrm{~B}$ & 31.3 & 4 & 5 & 150 \\
\hline
\end{tabular}

Peak Information

\begin{tabular}{|l|l|l|l|l|l|}
\hline Peak No & $\%$ Area & Area & Ret. Time & Height & Cap. Factor \\
\hline 1 & 4.834 & 317.8766 & $5.8 \mathrm{~min}$ & 22.2115 & 0 \\
\hline 2 & 95.166 & 6257.9892 & $6.71 \mathrm{~min}$ & 311.2555 & 0 \\
\hline
\end{tabular}




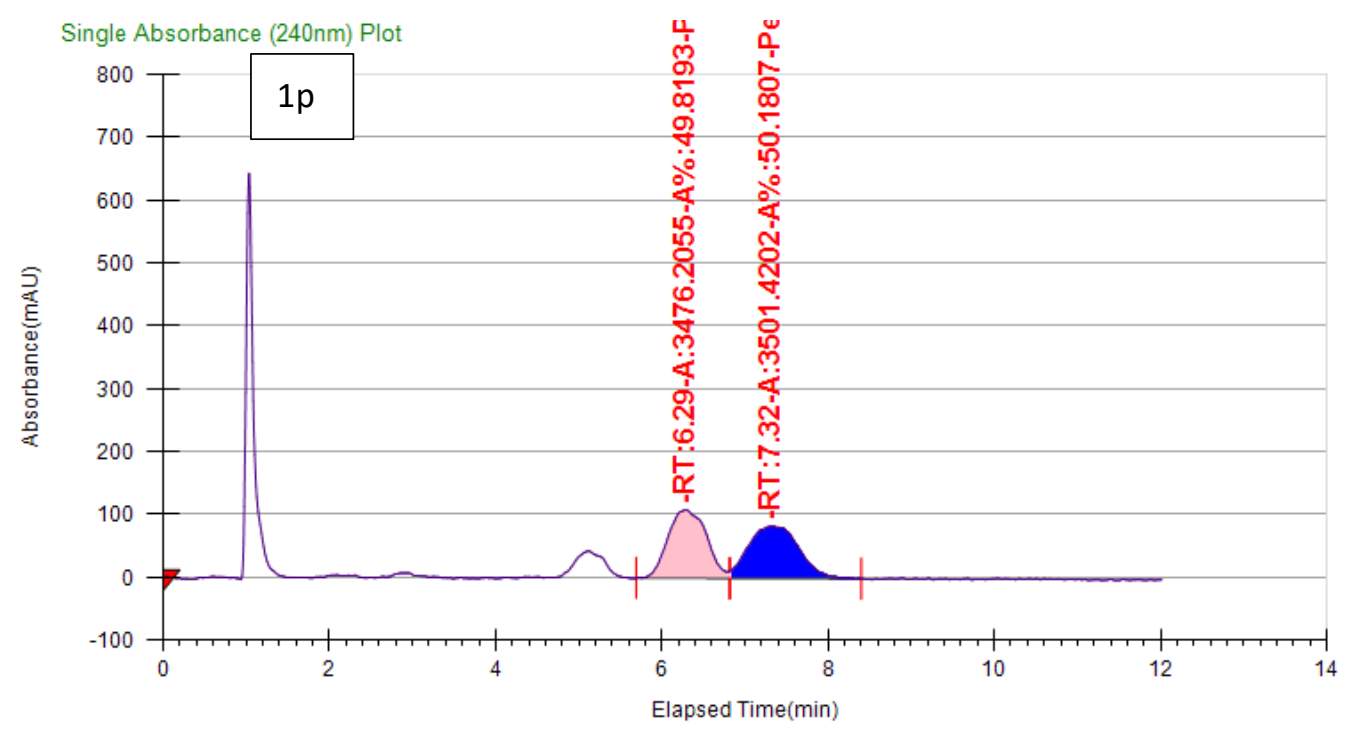

Run Information

\begin{tabular}{|l|l|l|l|l|l|l|l|l|l|}
\hline Instrument Method & Inj. Vol. & Solvent & Column & Sample & Well Location & Temp. & Flow & $\%$ Modifier & Pressure \\
\hline 20p methanol & 10 & MeOH & AD-H & $\begin{array}{l}\text { yp-hwu-6-40- } \\
\text { DL-AD20\% }\end{array}$ & $11 \mathrm{D}$ & 30 & 4 & 20 & 150 \\
\hline
\end{tabular}

\section{Peak Information}

\begin{tabular}{|l|l|l|l|l|l|}
\hline Peak No & $\%$ Area & Area & Ret. Time & Height & Cap. Factor \\
\hline 1 & 49.8193 & 3476.2055 & $6.29 \mathrm{~min}$ & 108.5372 & 6290.5833 \\
\hline 2 & 50.1807 & 3501.4202 & $7.32 \mathrm{~min}$ & 82.9654 & 7323.9 \\
\hline
\end{tabular}

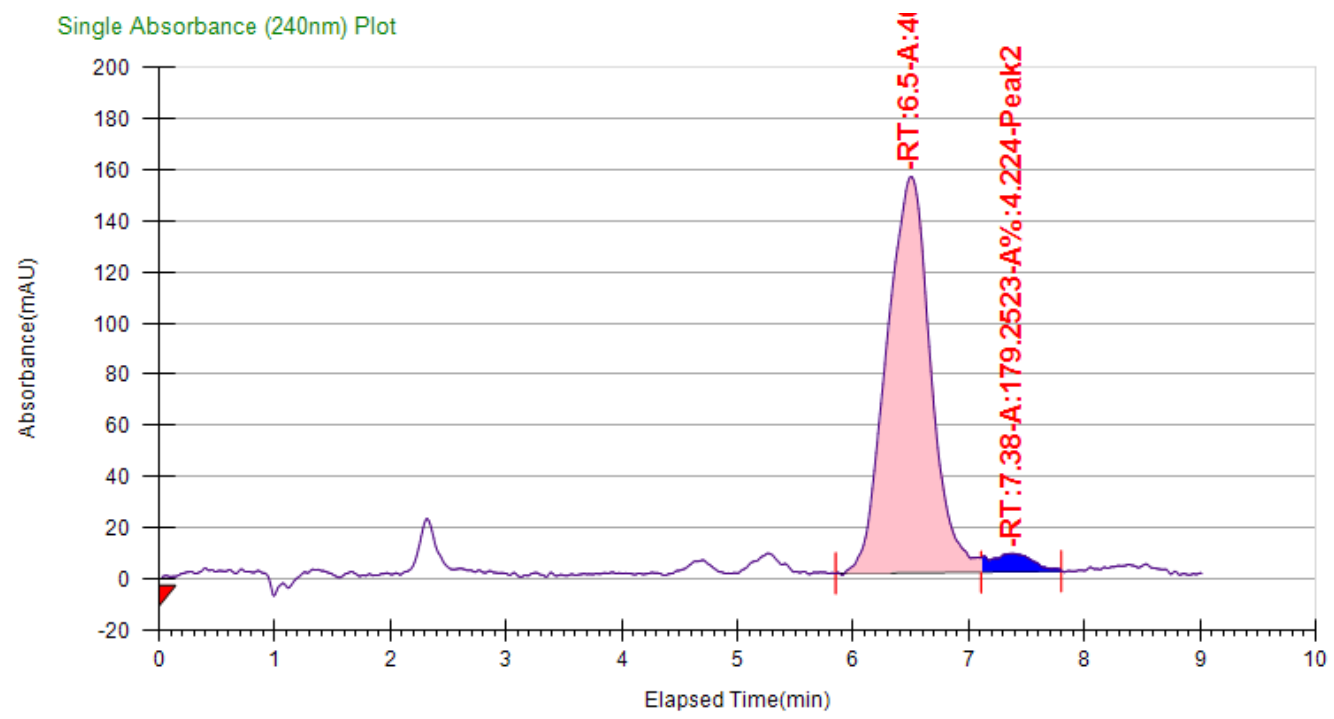

Run Information

\begin{tabular}{|l|l|l|l|l|l|l|l|l|l|}
\hline Instrument Method & Inj. Vol. & Solvent & Column & Sample & Well Location & Temp. & Flow & \% Modifier & Pressure \\
\hline 20p methanol & 10 & $\mathrm{MeOH}$ & AD-H & $\begin{array}{l}\text { yp-hwu-6-40- } \\
\text { asy-AD20\% }\end{array}$ & $11 \mathrm{C}$ & 29 & 4 & 20 & 150 \\
\hline
\end{tabular}

\section{Peak Information}

\begin{tabular}{|l|l|l|l|l|l|}
\hline Peak No & $\%$ Area & Area & Ret. Time & Height & Cap. Factor \\
\hline 1 & 95.776 & 4064.3617 & $6.5 \mathrm{~min}$ & 154.7575 & 0 \\
\hline 2 & 4.224 & 179.2523 & $7.38 \mathrm{~min}$ & 7.1007 & 0 \\
\hline
\end{tabular}




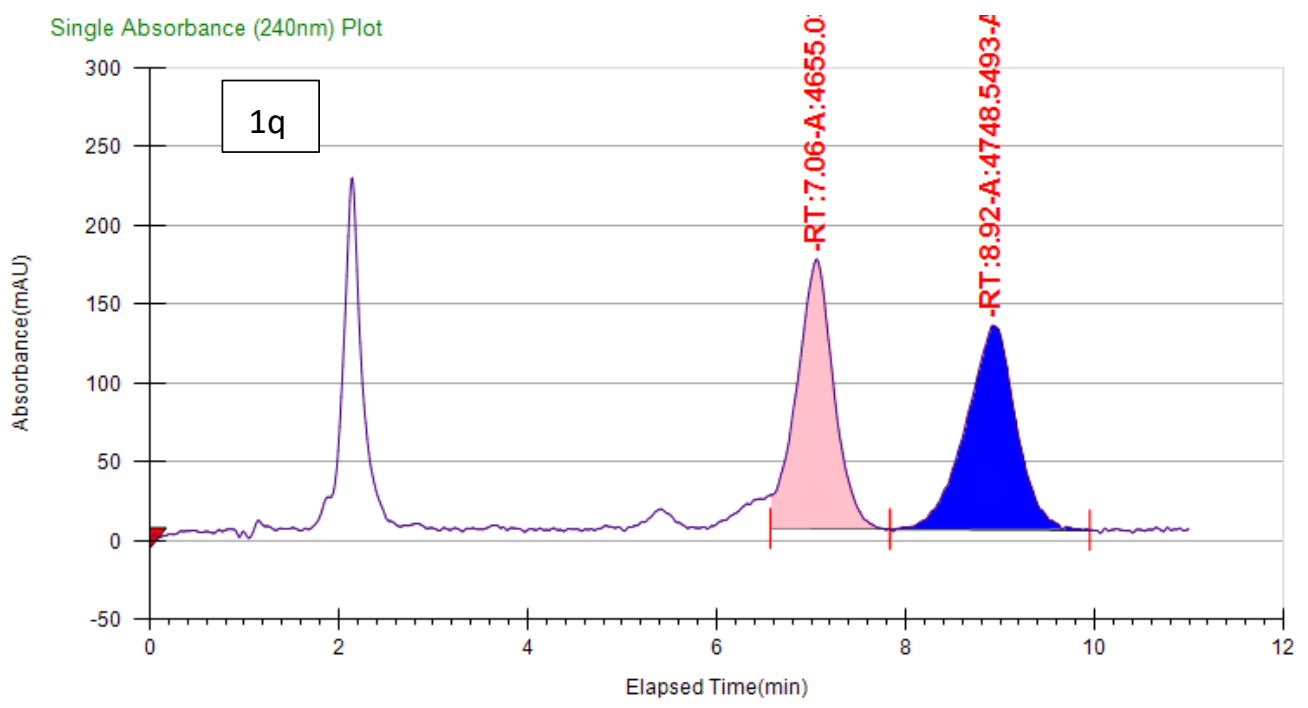

Run Information

\begin{tabular}{|l|l|l|l|l|l|l|l|l|l|}
\hline Instrument Method & Inj. Vol. & Solvent & Column & Sample & Well Location & Temp. & Flow & $\%$ Modifier & Pressure \\
\hline $5 p$ methanol & 10 & $\mathrm{MeOH}$ & AD-H & $\begin{array}{l}\text { yp-hwu-6-38- } \\
\text { 2OMe-DL- } \\
\text { AD5\% }\end{array}$ & $11 \mathrm{~A}$ & 29.5 & 4 & 5 & 150 \\
\hline
\end{tabular}

Peak Information

\begin{tabular}{|l|l|l|l|l|l|}
\hline Peak No & \% Area & Area & Ret. Time & Height & Cap. Factor \\
\hline 1 & 49.5028 & 4655.0332 & $7.06 \mathrm{~min}$ & 171.0985 & 7057.2333 \\
\hline 2 & 50.4972 & 4748.5493 & $8.92 \mathrm{~min}$ & 129.6589 & 8923.8667 \\
\hline
\end{tabular}

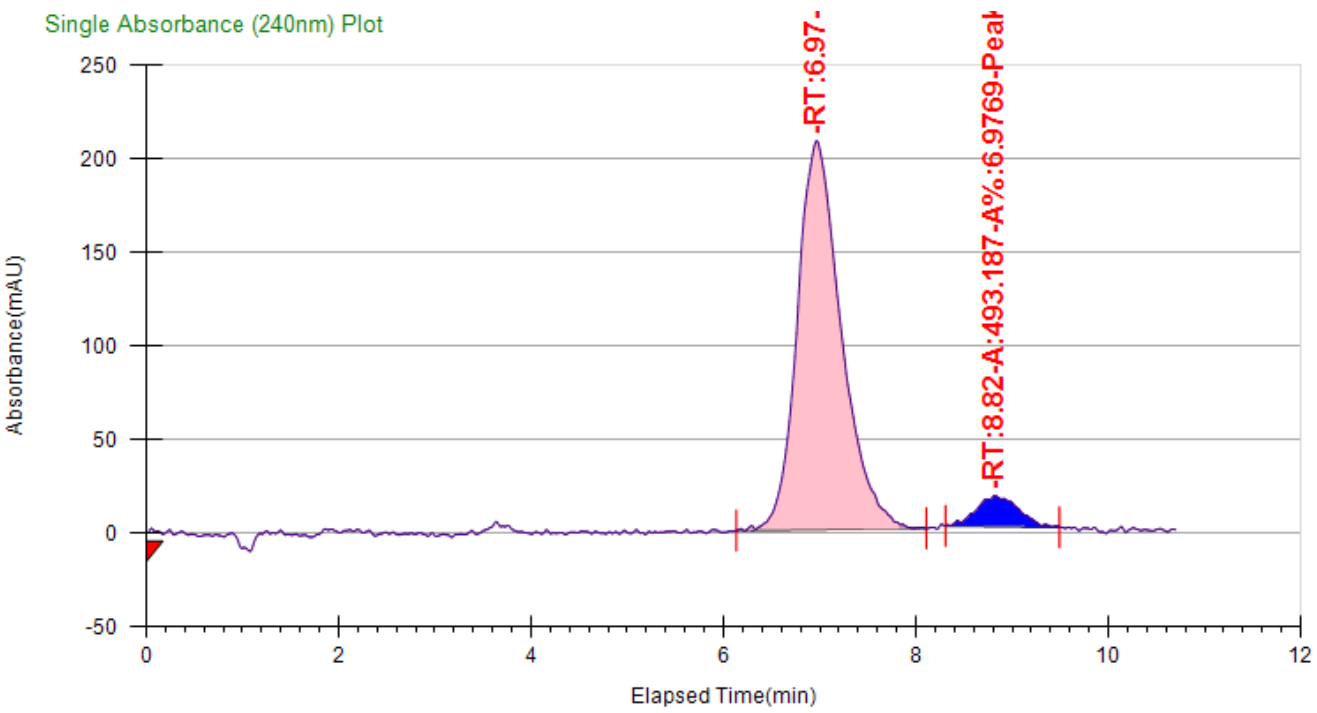

Run Information

\begin{tabular}{|l|l|l|l|l|l|l|l|l|l|}
\hline Instrument Method & Inj. Vol. & Solvent & Column & Sample & Well Location & Temp. & Flow & $\%$ Modifier & Pressure \\
\hline $5 p$ methanol & 10 & $\mathrm{MeOH}$ & AD-H & $\begin{array}{l}\text { yp-hwu-6-38- } \\
\text { 2OMe-asy- } \\
\text { AD510\% }\end{array}$ & $11 \mathrm{~B}$ & 28.9 & 4 & 5 & 150 \\
\hline
\end{tabular}

Peak Information

\begin{tabular}{|l|l|l|l|l|l|}
\hline Peak No & $\%$ Area & Area & Ret. Time & Height & Cap. Factor \\
\hline 1 & 93.0231 & 6575.6882 & $6.97 \mathrm{~min}$ & 207.6839 & 0 \\
\hline 2 & 6.9769 & 493.187 & $8.82 \mathrm{~min}$ & 16.5469 & 0 \\
\hline
\end{tabular}




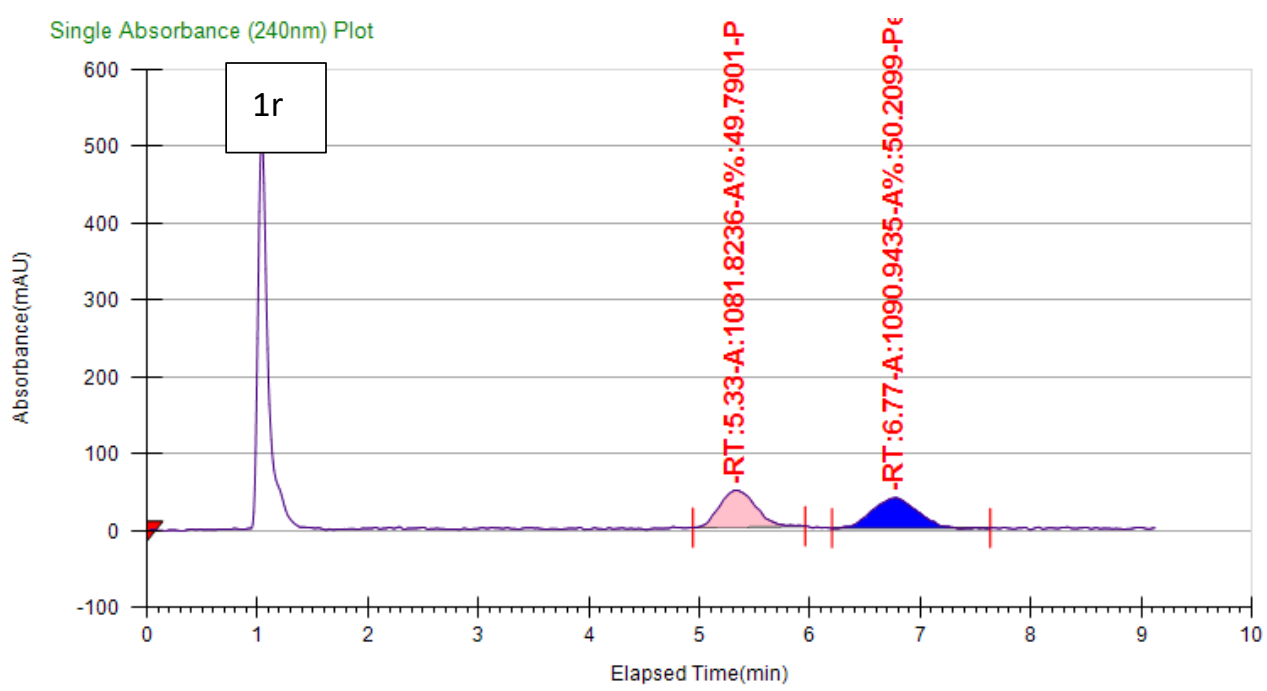

Run Information

\begin{tabular}{|l|l|l|l|l|l|l|l|l|l|}
\hline Instrument Method & Inj. Vol. & Solvent & Column & Sample & Well Location & Temp. & Flow & $\%$ Modifier & Pressure \\
\hline $5 p$ methanol & 10 & MeOH & AD-H & $\begin{array}{l}\text { yp-6-51-DL- } \\
\text { AD5\% }\end{array}$ & $11 \mathrm{~B}$ & 29.5 & 4 & 5 & 150 \\
\hline
\end{tabular}

\section{Peak Information}

\begin{tabular}{|l|l|l|l|l|l|}
\hline Peak No & $\%$ Area & Area & Ret. Time & Height & Cap. Factor \\
\hline 1 & 49.7901 & 1081.8236 & $5.33 \mathrm{~min}$ & 47.9009 & 0 \\
\hline 2 & 50.2099 & 1090.9435 & $6.77 \mathrm{~min}$ & 38.9324 & 0 \\
\hline
\end{tabular}

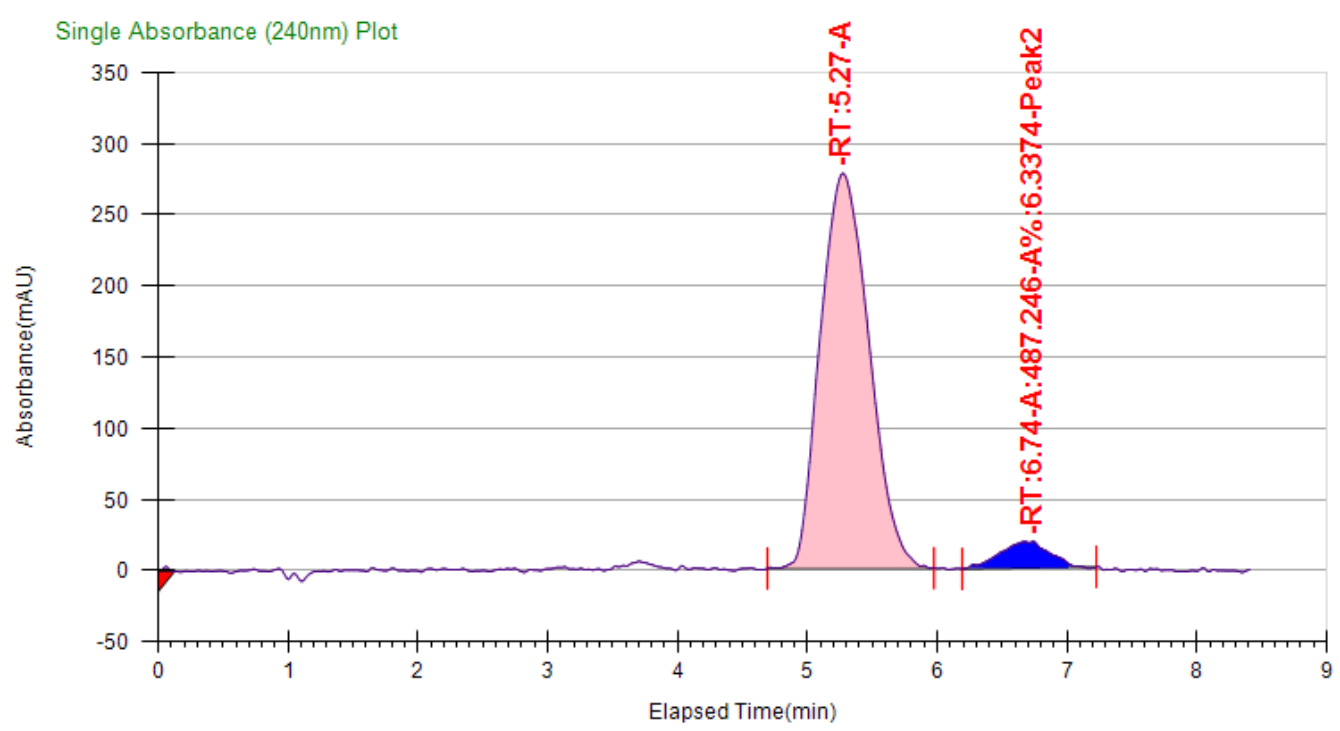

Run Information

\begin{tabular}{|l|l|l|l|l|l|l|l|l|l|}
\hline Instrument Method & Inj. Vol. & Solvent & Column & Sample & Well Location & Temp. & Flow & \% Modifier & Pressure \\
\hline $5 p$ methanol & 10 & $\mathrm{MeOH}$ & AD-H & $\begin{array}{l}\text { yp-6-51-asy- } \\
\text { AD5\% }\end{array}$ & $11 \mathrm{~A}$ & 30.3 & 4 & 5 & 150 \\
\hline
\end{tabular}

Peak Information

\begin{tabular}{|l|l|l|l|l|l|}
\hline Peak No & $\%$ Area & Area & Ret. Time & Height & Cap. Factor \\
\hline 1 & 93.6626 & 7201.1494 & $5.27 \mathrm{~min}$ & 277.7984 & 0 \\
\hline 2 & 6.3374 & 487.246 & $6.74 \mathrm{~min}$ & 18.5413 & 0 \\
\hline
\end{tabular}




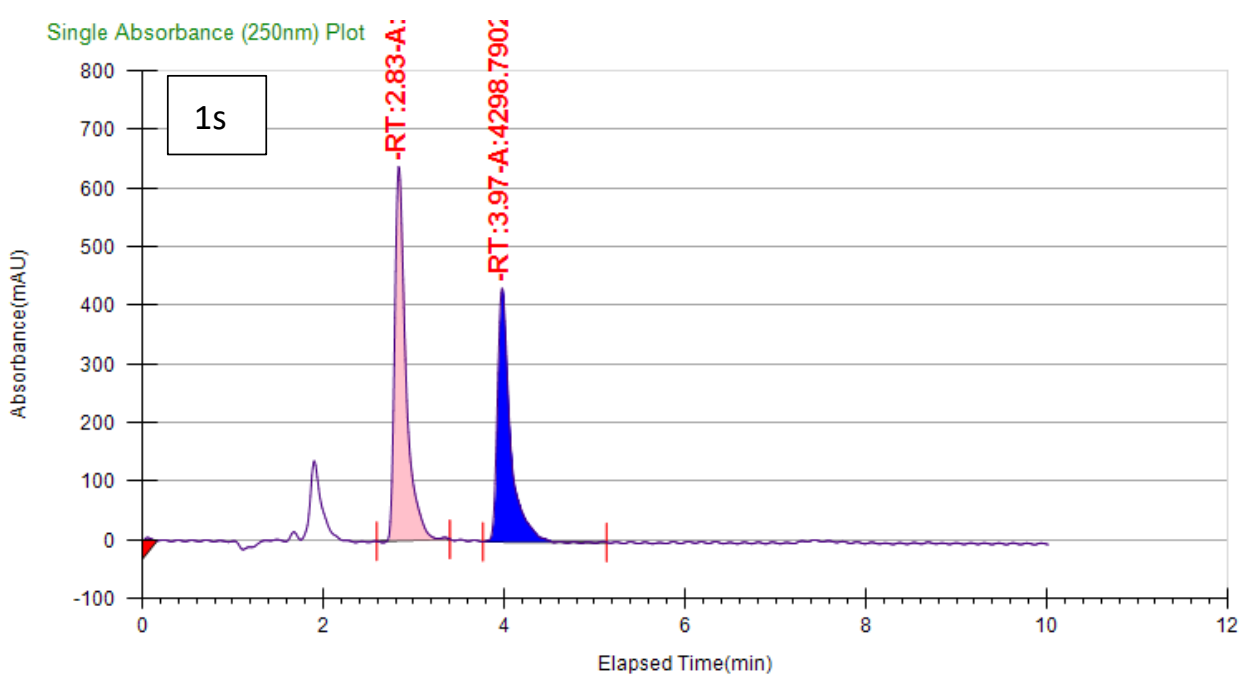

Run Information

\begin{tabular}{|l|l|l|l|l|l|l|l|l|l|}
\hline Instrument Method & Inj. Vol. & Solvent & Column & Sample & Well Location & Temp. & Flow & $\%$ Modifier & Pressure \\
\hline 20p methanol & 10 & $\mathrm{MeOH}$ & IA & $\begin{array}{l}6-122-2- \\
\text { DL'IA20\% }\end{array}$ & $11 \mathrm{~A}$ & 29.9 & 4 & 20 & 150 \\
\hline
\end{tabular}

Peak Information

\begin{tabular}{|l|l|l|l|l|l|}
\hline Peak No & \% Area & Area & Ret. Time & Height & Cap. Factor \\
\hline 1 & 56.1729 & 5509.7223 & $2.83 \mathrm{~min}$ & 638.0525 & 0 \\
\hline 2 & 43.8271 & 4298.7902 & $3.97 \mathrm{~min}$ & 432.5734 & 0 \\
\hline
\end{tabular}

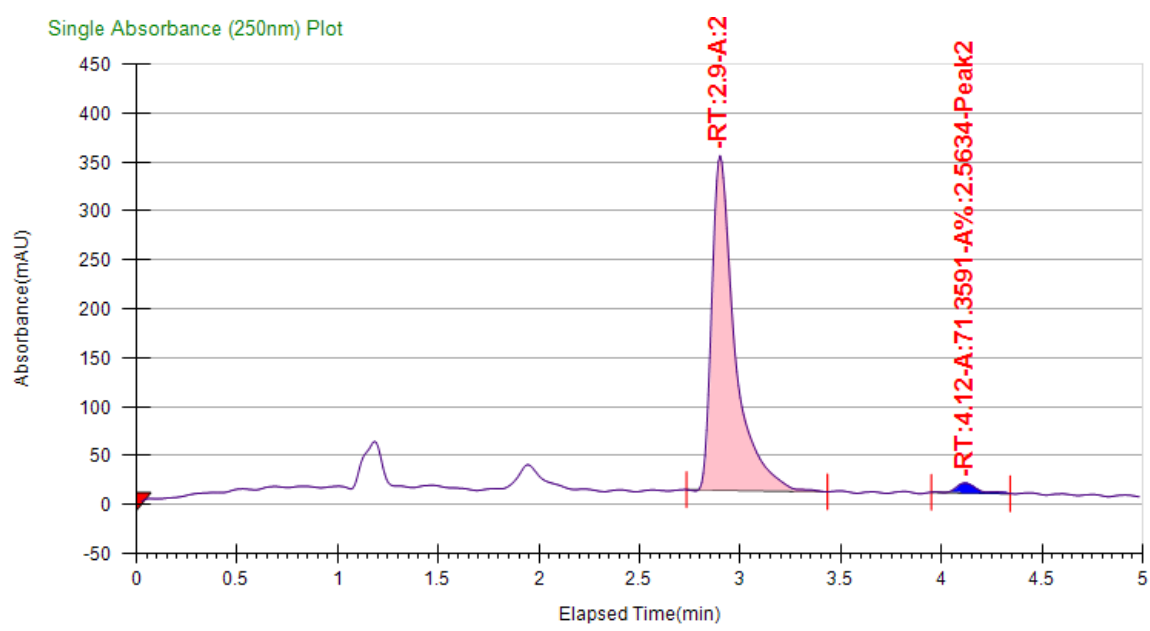

Run Information

\begin{tabular}{|l|l|l|l|l|l|l|l|l|l|}
\hline Instrument Method & Inj. Vol. & Solvent & Column & Sample & Well Location & Temp. & Flow & $\%$ Modifier & Pressure \\
\hline $20 \mathrm{p}$ methanol & 10 & $\mathrm{MeOH}$ & IA & $\begin{array}{l}6-122- \\
\text { 2'asz'IA20\% }\end{array}$ & $11 \mathrm{C}$ & 29.1 & 4 & 20 & 150 \\
\hline
\end{tabular}

Peak Information

\begin{tabular}{|l|l|l|l|l|l|}
\hline Peak No & $\%$ Area & Area & Ret. Time & Height & Cap. Factor \\
\hline 1 & 97.4366 & 2712.4439 & $2.9 \mathrm{~min}$ & 341.7246 & 0 \\
\hline 2 & 2.5634 & 71.3591 & $4.12 \mathrm{~min}$ & 9.9865 & 0 \\
\hline
\end{tabular}




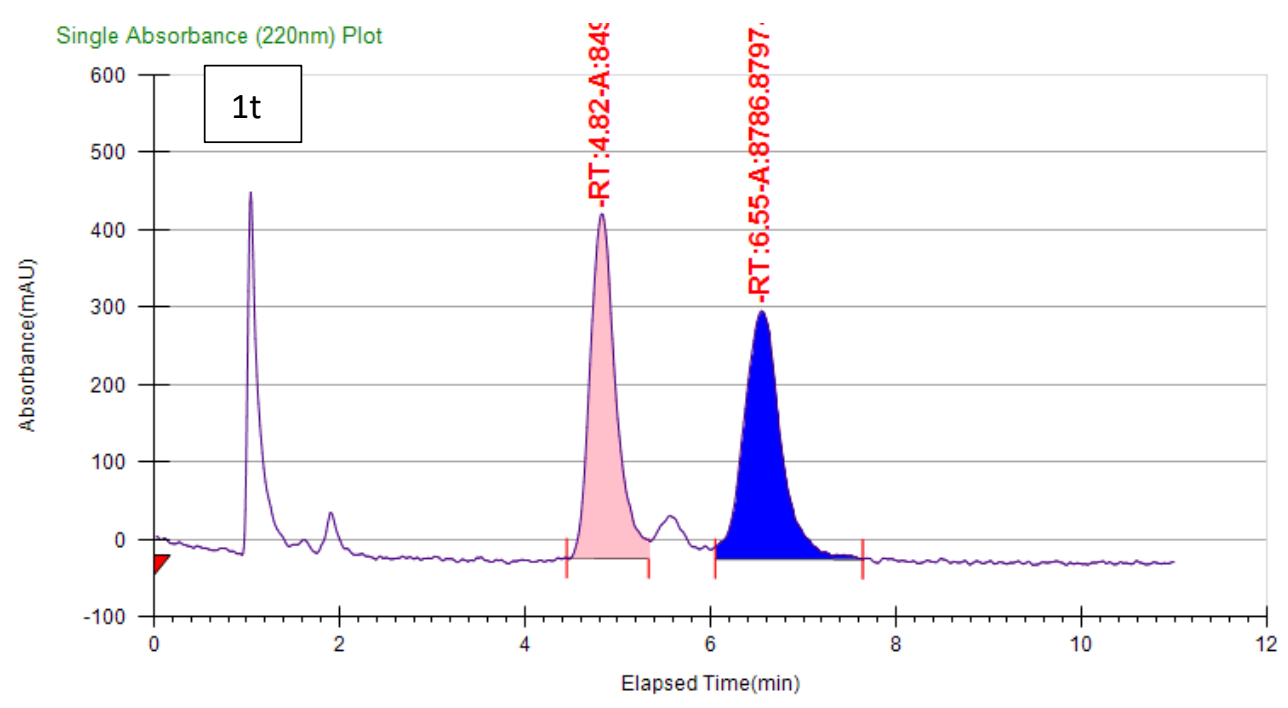

Run Information

\begin{tabular}{|l|l|l|l|l|l|l|l|l|l|}
\hline Instrument Method & Inj. Vol. & Solvent & Column & Sample & Well Location & Temp. & Flow & $\%$ Modifier & Pressure \\
\hline 10p methanol & 10 & $\mathrm{MeOH}$ & AD-H & $\begin{array}{l}\text { yp-6-57-DL- } \\
\text { AD10\% }\end{array}$ & $11 \mathrm{~A}$ & 30.1 & 4 & 10 & 150 \\
\hline
\end{tabular}

Peak Information

\begin{tabular}{|l|l|l|l|l|l|}
\hline Peak No & $\%$ Area & Area & Ret. Time & Height & Cap. Factor \\
\hline 1 & 49.1525 & 8493.9545 & $4.82 \mathrm{~min}$ & 445.0992 & 4823.9333 \\
\hline 2 & 50.8475 & 8786.8797 & $6.55 \mathrm{~min}$ & 320.502 & 6548.9 \\
\hline
\end{tabular}

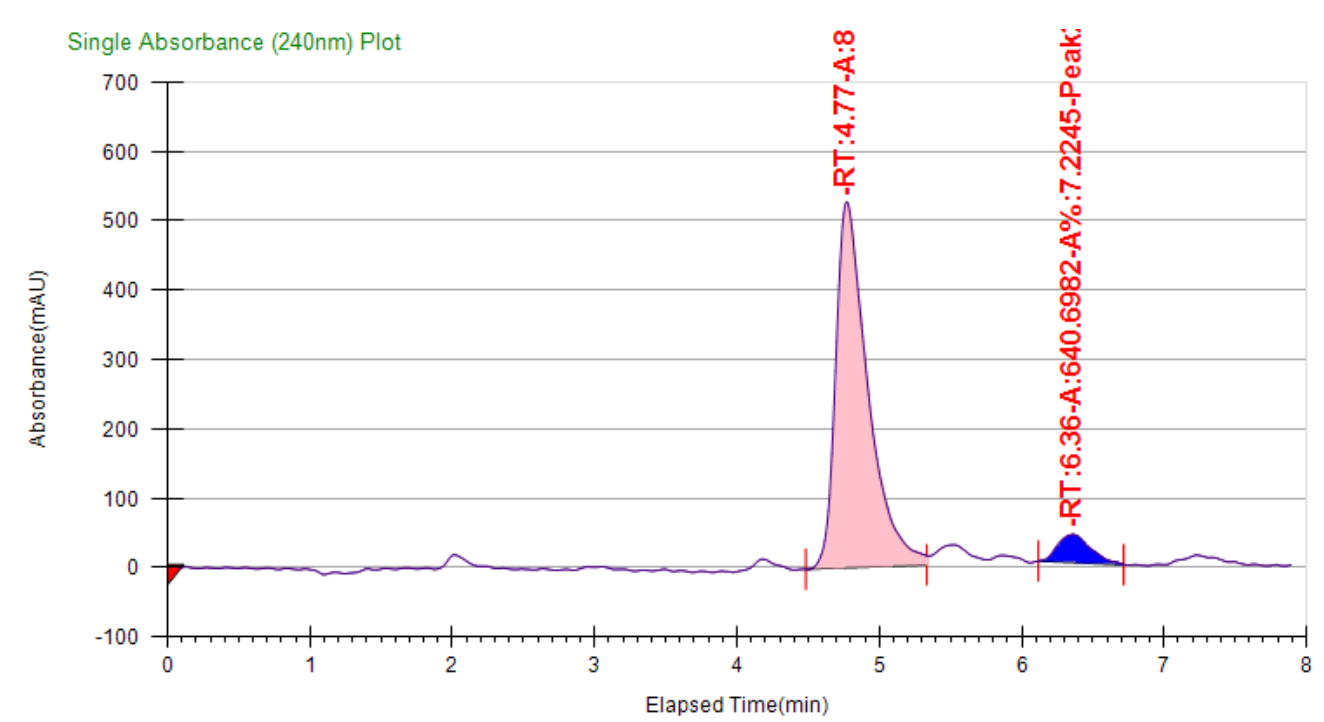

Run Information

\begin{tabular}{|c|c|c|c|c|c|c|c|c|c|}
\hline Instrument Method & Inj. Vol. & Solvent & Column & Sample & Well Location & Temp. & Flow & $\%$ Modifier & Pressure \\
\hline 10p methanol & 10 & $\mathrm{MeOH}$ & AD-H & $\begin{array}{l}\text { 6-57-1'60oC-- } \\
\text { AD10\% }\end{array}$ & 11D & 28.1 & 4 & 10 & 150 \\
\hline
\end{tabular}

Peak Information

\begin{tabular}{|l|l|l|l|l|l|}
\hline Peak No & \% Area & Area & Ret. Time & Height & Cap. Factor \\
\hline 1 & 92.7755 & 8227.6729 & $4.77 \mathrm{~min}$ & 527.7273 & 0 \\
\hline 2 & 7.2245 & 640.6982 & $6.36 \mathrm{~min}$ & 40.3235 & 0 \\
\hline
\end{tabular}




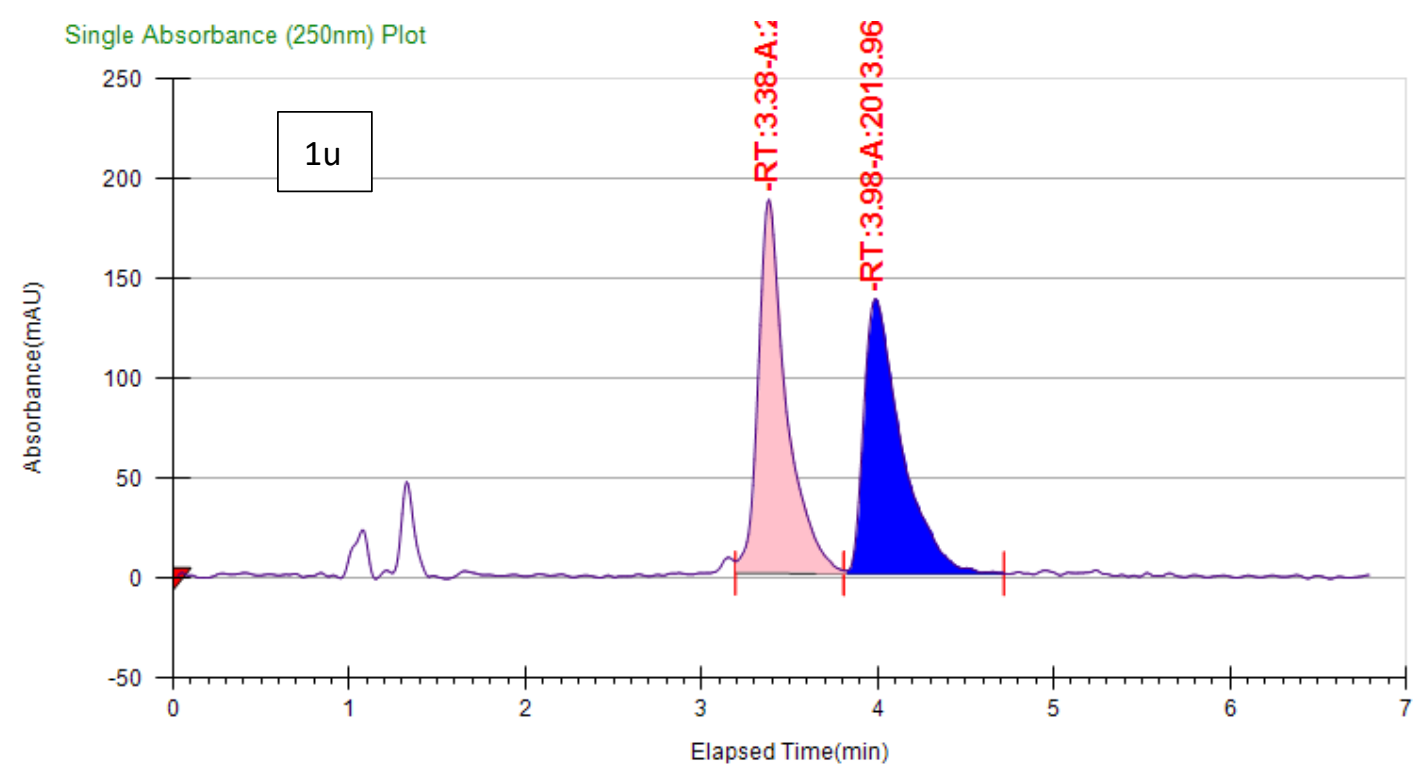

Run Information

\begin{tabular}{|l|l|l|l|l|l|l|l|l|l|}
\hline Instrument Method & Inj. Vol. & Solvent & Column & Sample & Well Location & Temp. & Flow & $\%$ Modifier & Pressure \\
\hline $10 p$ methanol & 10 & MeOH & OD-H & $\begin{array}{l}\text { yp-6-55- } \\
\text { DL'OD } 10 \%\end{array}$ & $11 \mathrm{~F}$ & 29.9 & 4 & 10 & 150 \\
\hline
\end{tabular}

\section{Peak Information}

\begin{tabular}{|l|l|l|l|l|l|}
\hline Peak No & $\%$ Area & Area & Ret. Time & Height & Cap. Factor \\
\hline 1 & 50.6057 & 2063.359 & $3.38 \mathrm{~min}$ & 187.1047 & 3382.2833 \\
\hline 2 & 49.3943 & 2013.964 & $3.98 \mathrm{~min}$ & 137.3465 & 3982.2833 \\
\hline
\end{tabular}

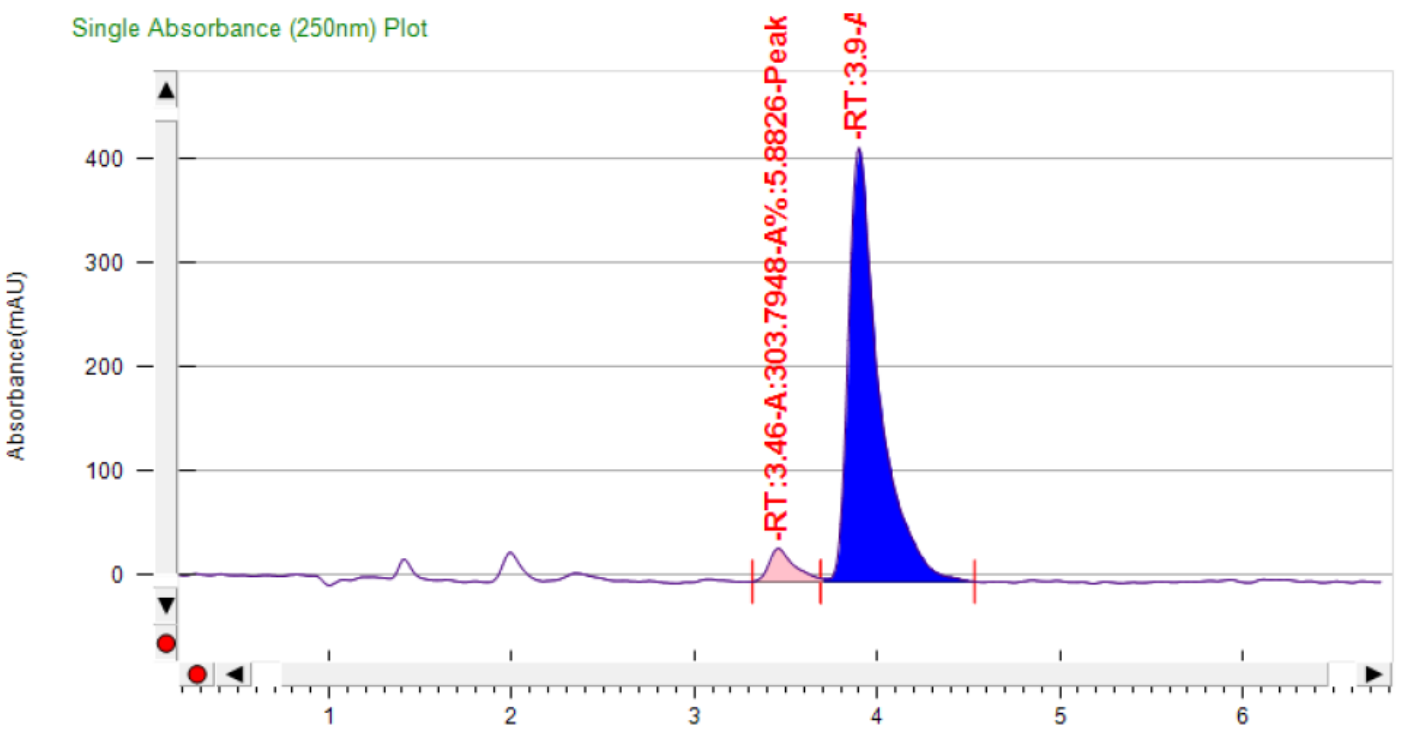

Run Information

\begin{tabular}{|l|l|l|l|l|l|l|l|l|l|}
\hline Instrument Method & Inj. Vol. & Solvent & Column & Sample & Well Location & Temp. & Flow & $\%$ Modifier & Pressure \\
\hline 10p methanol & 10 & MeOH & OD-H & $\begin{array}{l}6-555^{6} 60 \mathrm{OC}- \\
\text { ODD10\% }\end{array}$ & $11 \mathrm{~F}$ & 28.8 & 4 & 10 & 150 \\
\hline
\end{tabular}

Peak Information

\begin{tabular}{|l|l|l|l|l|l|}
\hline Peak No & \% Area & Area & Ret. Time & Height & Cap. Factor \\
\hline 1 & 5.8826 & 303.7948 & $3.46 \mathrm{~min}$ & 32.0159 & 3457.2833 \\
\hline 2 & 94.1174 & 4860.4775 & $3.9 \mathrm{~min}$ & 418.5267 & 3898.95 \\
\hline
\end{tabular}




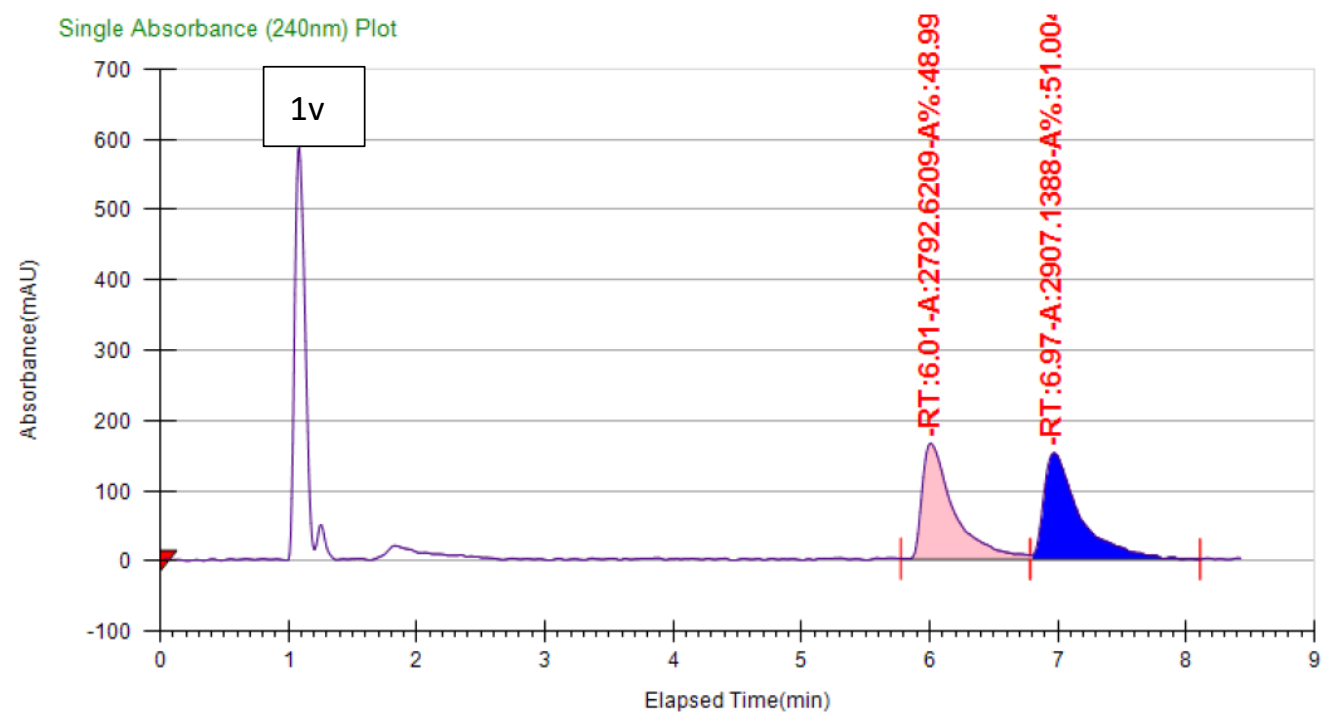

Run Information

\begin{tabular}{|l|l|l|l|l|l|l|l|l|l|}
\hline Instrument Method & Inj. Vol. & Solvent & Column & Sample & Well Location & Temp. & Flow & \% Modifier & Pressure \\
\hline $3 p$ methanol & 10 & $\mathrm{MeOH}$ & IA & $\begin{array}{l}\text { yp-6-54'-DL- } \\
\text { IA3\% }\end{array}$ & $11 \mathrm{E}$ & 29.9 & 4 & 3 & 150 \\
\hline
\end{tabular}

Peak Information

\begin{tabular}{|l|l|l|l|l|l|}
\hline Peak No & $\%$ Area & Area & Ret. Time & Height & Cap. Factor \\
\hline 1 & 48.9954 & 2792.6209 & $6.01 \mathrm{~min}$ & 164.4112 & 6007.25 \\
\hline 2 & 51.0046 & 2907.1388 & $6.97 \mathrm{~min}$ & 150.842 & 6965.5667 \\
\hline
\end{tabular}

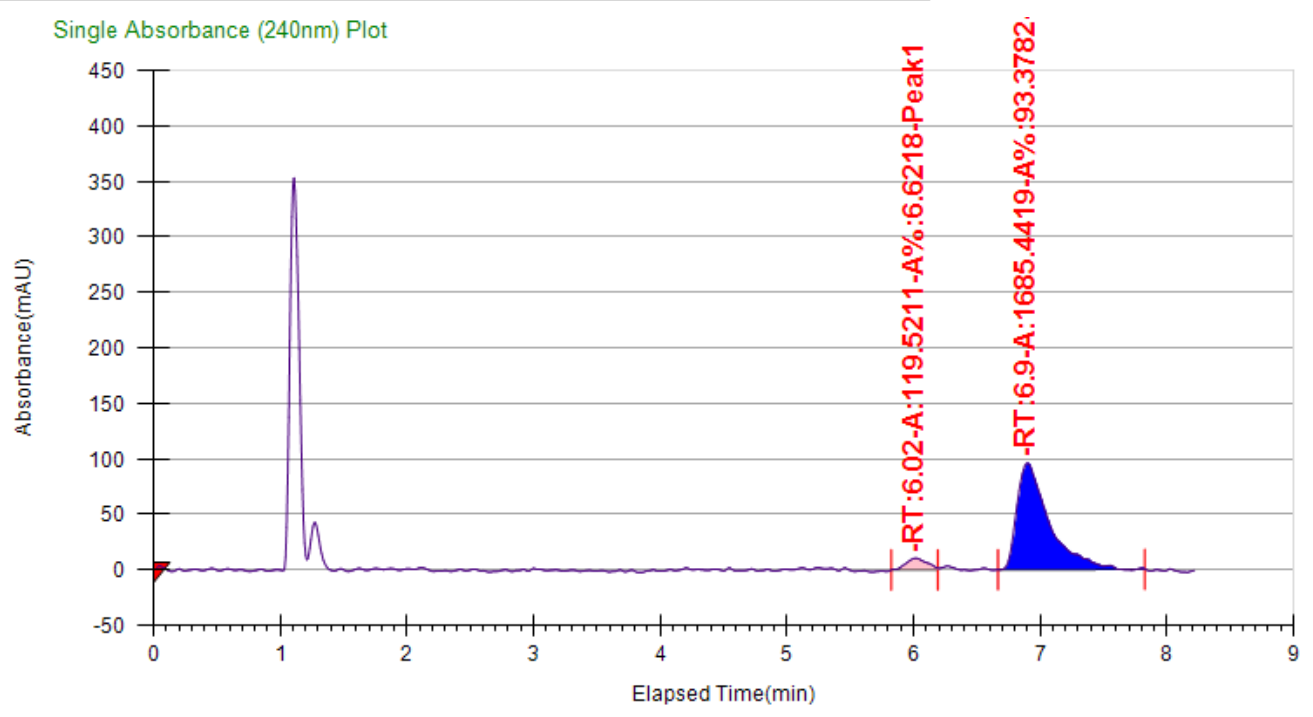

Run Information

\begin{tabular}{|l|l|l|l|l|l|l|l|l|l}
\hline Instrument Method & Inj. Vol. & Solvent & Column & Sample & Well Location & Temp. & Flow & $\%$ Modifier & Pressure \\
\hline $3 p$ methanol & 10 & $\mathrm{MeOH}$ & IA & $\begin{array}{l}\text { yp-6-54- } \\
40 \circ C^{\prime} 1 \text { day- } \\
\text { IA3\% }\end{array}$ & $11 \mathrm{~A}$ & 31.2 & 4 & 3 & 150 \\
\hline
\end{tabular}

Peak Information

\begin{tabular}{|l|l|l|l|l|l|}
\hline Peak No & $\%$ Area & Area & Ret. Time & Height & Cap. Factor \\
\hline 1 & 6.6218 & 119.5211 & $6.02 \mathrm{~min}$ & 10.5218 & 0 \\
\hline 2 & 93.3782 & 1685.4419 & $6.9 \mathrm{~min}$ & 95.9471 & 0 \\
\hline
\end{tabular}




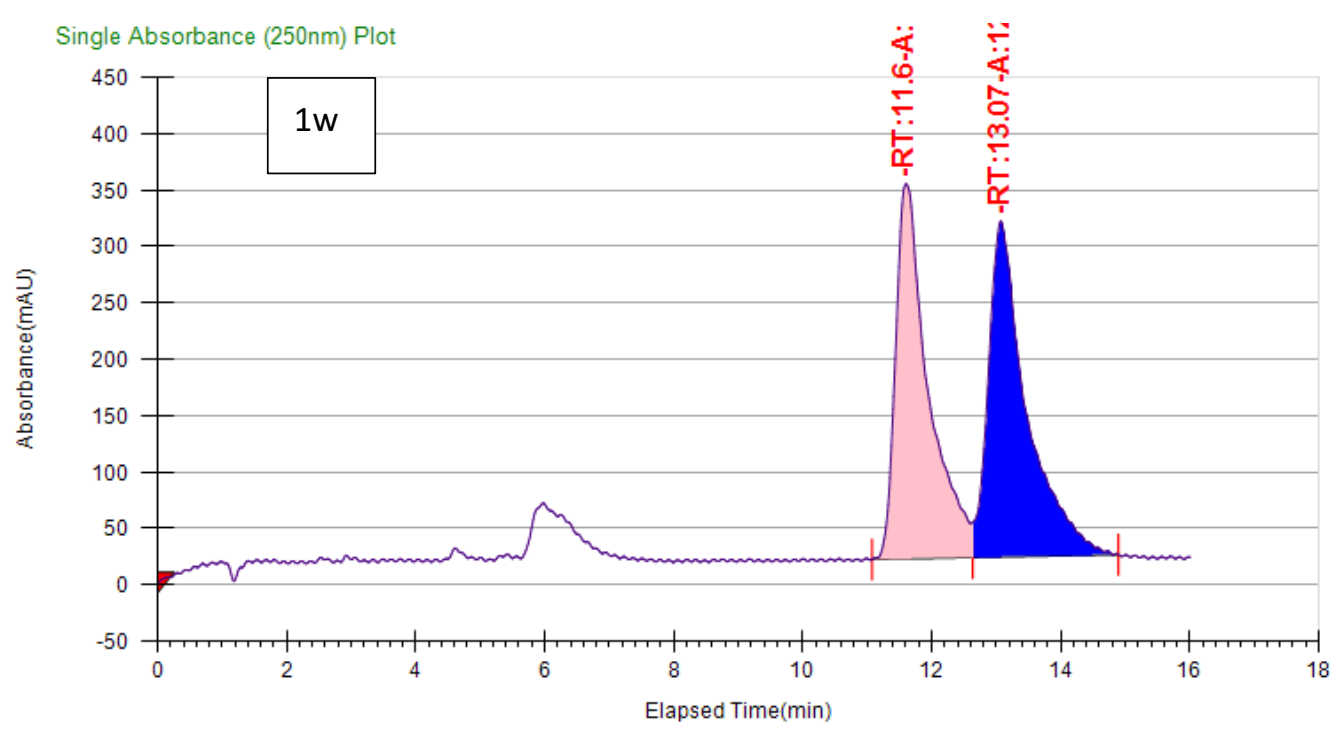

Run Information

\begin{tabular}{|l|l|l|l|l|l|l|l|l|l|}
\hline Instrument Method & Inj. Vol. & Solvent & Column & Sample & Well Location & Temp. & Flow & \%odifier & Pressure \\
\hline $10 \mathrm{p}$ methanol & 10 & $\mathrm{MeOH}$ & $\mathrm{OD}-\mathrm{H}$ & $\begin{array}{l}6-122- \\
1^{\prime} \mathrm{DL} \text { 'OD } 10 \%\end{array}$ & $11 \mathrm{E}$ & 29.6 & 4 & 10 & 150 \\
\hline
\end{tabular}

Peak Information

\begin{tabular}{|l|l|l|l|l|l|}
\hline Peak No & $\%$ Area & Area & Ret. Time & Height & Cap. Factor \\
\hline 1 & 48.9898 & $\begin{array}{l}11681.062 \\
7\end{array}$ & $11.6 \mathrm{~min}$ & 332.341 & 0 \\
\hline 2 & 51.0102 & 12162.803 & $13.07 \mathrm{~min}$ & 297.9113 & 0 \\
\hline
\end{tabular}

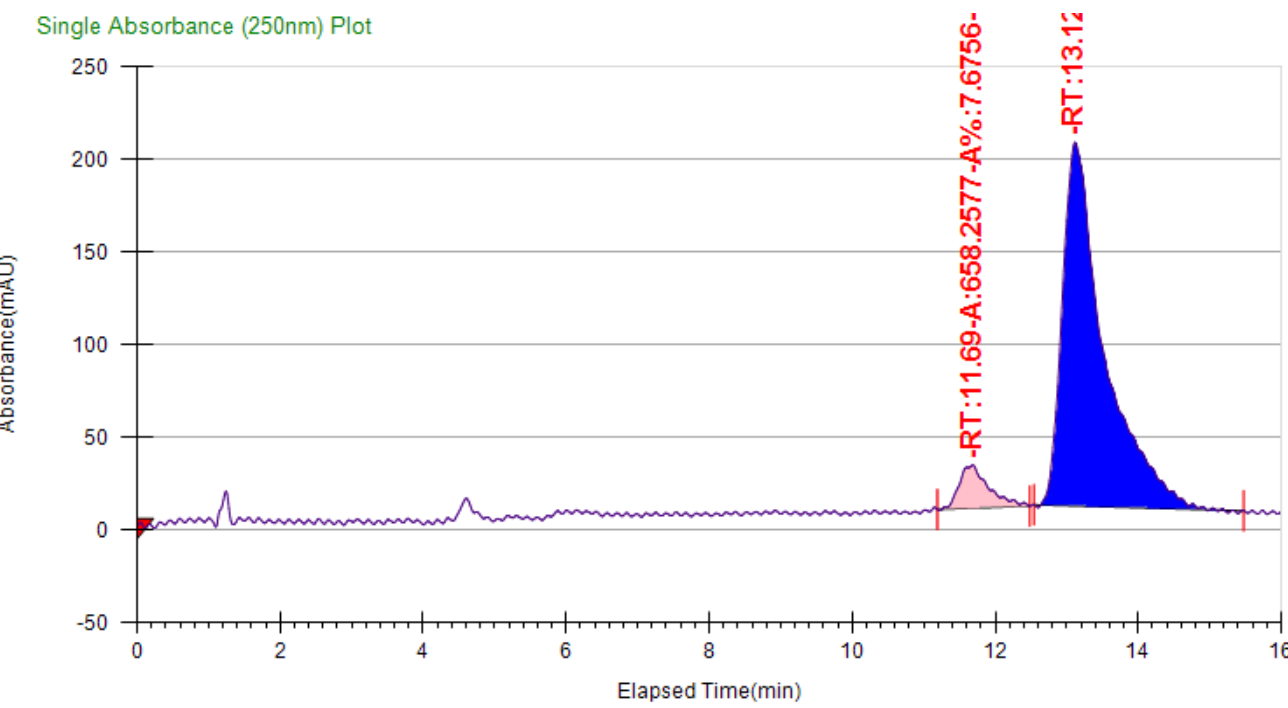

Run Information

\begin{tabular}{|l|l|l|l|l|l|l|l|l|l|}
\hline Instrument Method & Inj. Vol. & Solvent & Column & Sample & Well Location & Temp. & Flow & $\%$ Modifier & Pressure \\
\hline $10 p$ methanol & 10 & $\mathrm{MeOH}$ & OD-H & $\begin{array}{l}6-122- \\
1 \text { 'asz'OD10\% }\end{array}$ & $11 \mathrm{D}$ & 30.1 & 4 & 10 & 150 \\
\hline
\end{tabular}

Peak Information

\begin{tabular}{|l|l|l|l|l|l|}
\hline Peak No & \% Area & Area & Ret. Time & Height & Cap. Factor \\
\hline 1 & 7.6756 & 658.2577 & $11.69 \mathrm{~min}$ & 23.2426 & 0 \\
\hline 2 & 92.3244 & 7917.7325 & $13.12 \mathrm{~min}$ & 196.2266 & 0 \\
\hline
\end{tabular}

